\title{
Microstructure-Based Computational Fatigue Life Prediction of Structural Materials
}

\author{
By
}

\section{Siqi Li}

A thesis submitted to the Faculty of Graduate and Postdoctoral Affairs in partial fulfillment of the requirements for the degree of

\section{Master of Applied Science in}

Mechanical Engineering

Carleton University

Ottawa, Ontario

C) 2020, Siqi Li 


\begin{abstract}
Conventionally, engineers have to perform fatigue testing, either in stress or in strain-controlled mode, to determine the fatigue properties of a material, which costs a great deal of time and money. Therefore, the concept of computational fatigue design has been proposed and received an increasing interest in recent years. In this research, a microstructure-based computational fatigue design model, named TMW model, is further studied first by using it to predict the fatigue crack nucleation lives of eight different alloys and steels, and comparing the predicted lives with the calculated values from the CoffinManson-Basquin relations which are obtained from experimental data fitting. Second, this model is improved by developing the mathematical expressions of the surface roughness factor in the TMW model in terms of the arithmetical mean deviation of the assessed profile which can be determined experimentally, thus making the TMW model more applicable. In addition, a microstructure-based finite element analysis (FEA) model is created to investigate the effect of microstructural inhomogeneity (grain orientation) on the fatigue crack nucleation life of nickel-based alloy Haynes 282 in different strain ranges from low cycle fatigue (LCF) to high cycle fatigue (HCF) at different stress amplitudes. Grain orientations are randomly assigned to a material representative volume element (RVE) with 20 random functions created for both HCF and LCF simulations. The TMW model shows effectiveness for predicting the fatigue crack nucleation life of structural materials. The FEA simulation reveals that potential fatigue crack nucleation sites are likely to occur at
\end{abstract}


grain boundaries right on or close to the free surface of the RVE. For both HCF and LCF cases, the simulated fatigue crack nucleation in Haynes 282 is more likely to occur in the grains of $\left[\begin{array}{lll}\overline{1} & 1 & 1\end{array}\right]$ family, while the grains with the least probability of fatigue crack nucleation tend to be oriented towards [ $\left[\begin{array}{lll}0 & 0 & 1\end{array}\right]$ family. 


\section{Acknowledgements}

Firstly, I wish to express my deepest gratitude to my thesis supervisor, Professor Rong Liu, for providing me the opportunity to study on such meaningful project. Furthermore, I would like to immensely thank my co-supervisors, Dr. Xijia Wu, and Dr. Zhong Zhang, for their valuable assistance and guidance on fatigue modeling and FEM sections of my thesis.

I also intend to extend my appreciation to the research assistant, Dr. Xiaozhou Zhang, as well as PhD students Yingping Ding and Peter Walker, for their kind help throughout my master program.

Finally, I wish to pay my special regards to my families, for their continuous support and encouragement to my academic career. 


\section{Table of Contents}

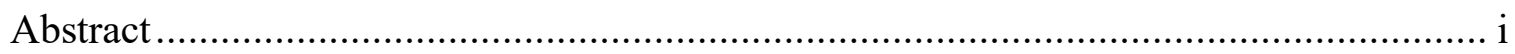

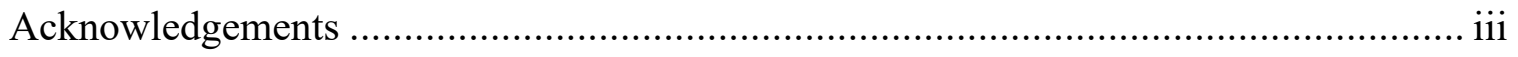

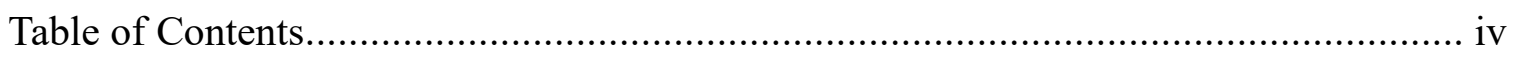

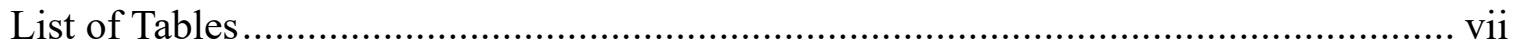

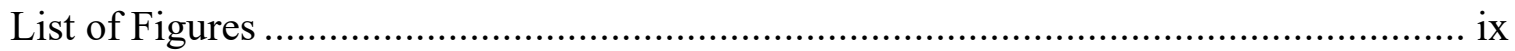

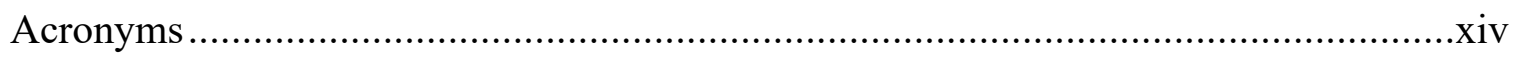

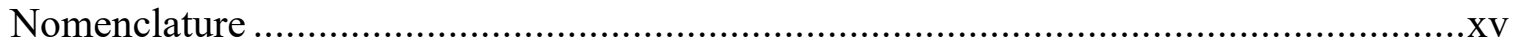

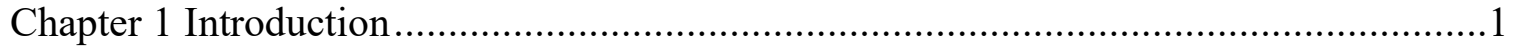

1.1 Background of the Research....................................................................... 1

1.2 Objectives of the Research .................................................................... 4

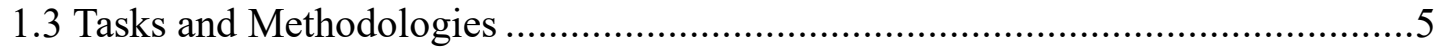

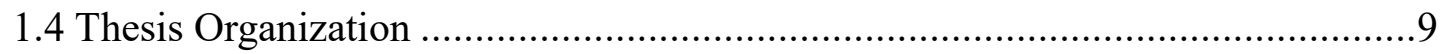

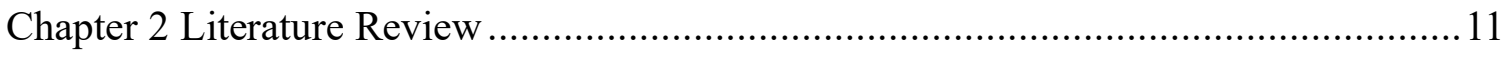

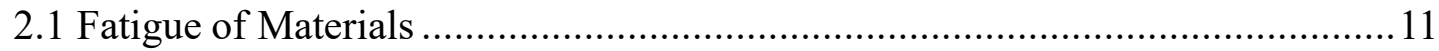

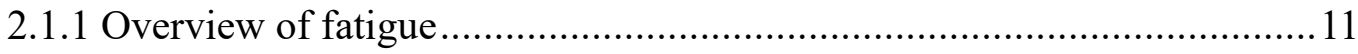

2.1.2 Fatigue crack nucleation .............................................................20

2.2 Prediction of Fatigue Crack Nucleation Life.................................................24

2.2.1 Models of fatigue life prediction .................................................24

2.2.2 Fatigue life prediction with FEM or FEA.........................................34

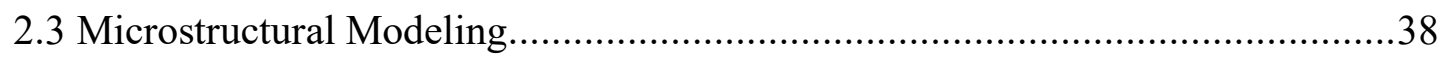

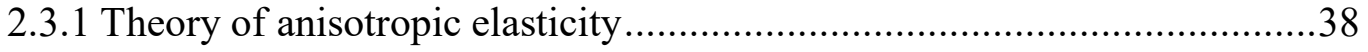

2.3.2 Yielding criteria and hardening law ..............................................40

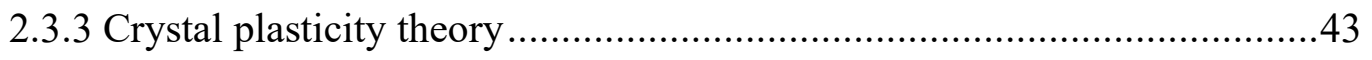

2.3.3.1 Definitions of crystallography .............................................43

2.3.3.2 Single crystal plasticity .................................................... 46

2.3.4 Representation of polycrystalline structure ......................................50

2.3.4.1 Representative volume element (RVE) ...................................5

2.3.4.2 Voronoi tessellation...............................................................51 


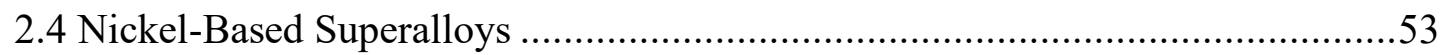

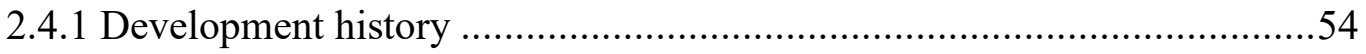

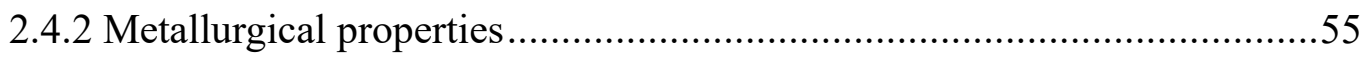

Chapter 3 Fatigue Life Prediction with the TMW Model...............................................62

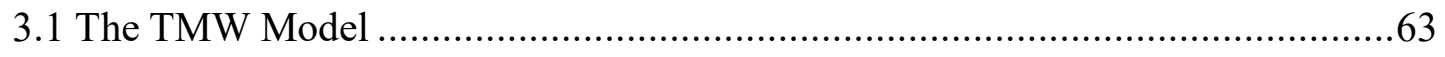

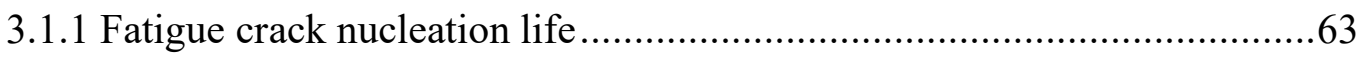

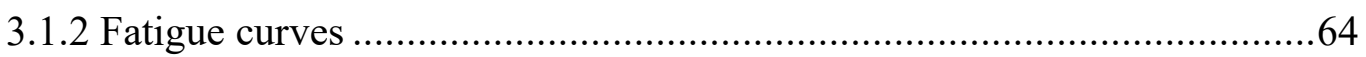

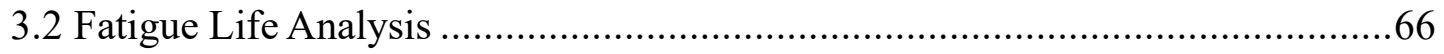

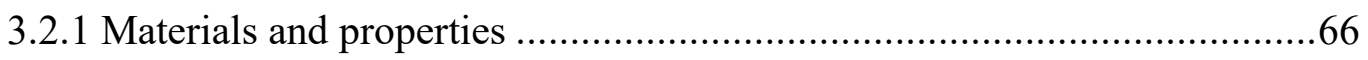

3.2.2 Parameters of the TMW model .............................................................

3.2.3 Fatigue curves for LCF and HCF ......................................................

3.3 Surface Roughness Factor in the TMW Model ................................................95

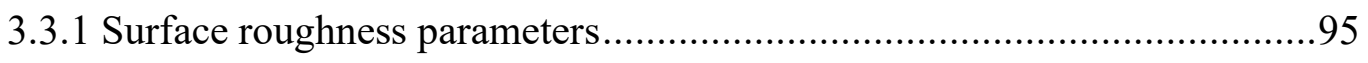

3.3.2 Influence of surface roughness on fatigue life ……………....................96

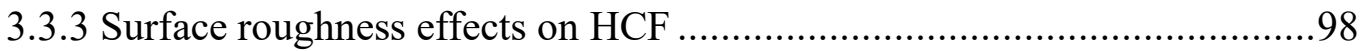

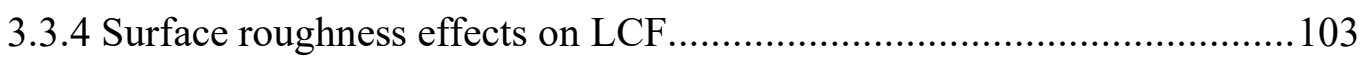

Chapter 4 FEA Simulation of Fatigue Crack Nucleation .............................................. 108

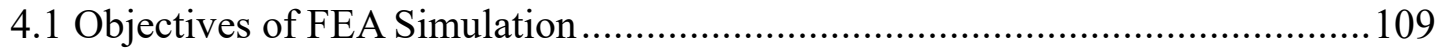

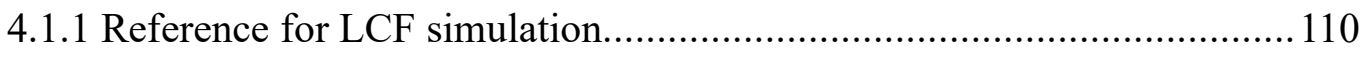

4.1.2 Reference for HCF simulation ............................................................. 112

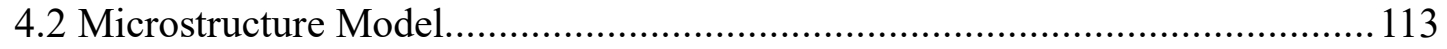

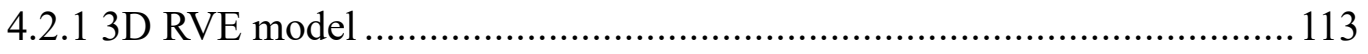

4.2.2 Grain orientation and inverse pole figure ............................................ 116

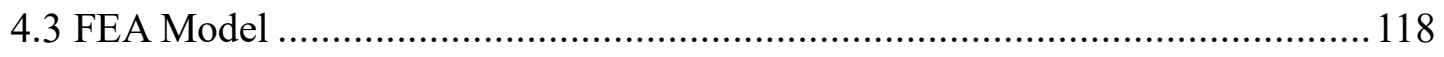

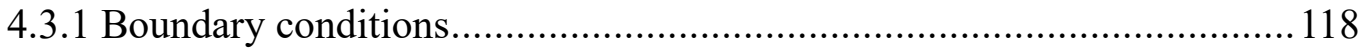

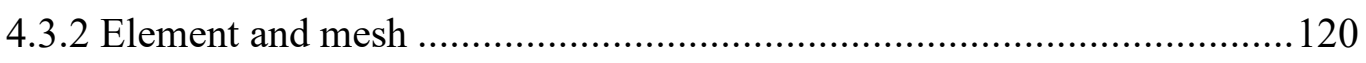

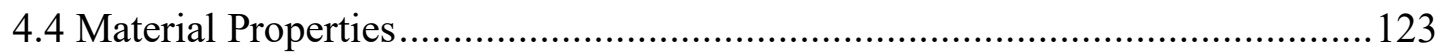

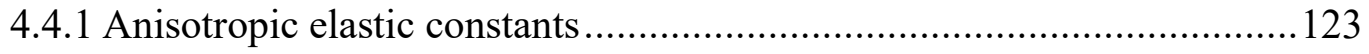

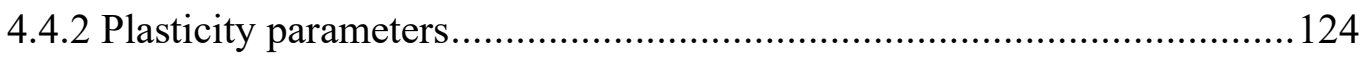

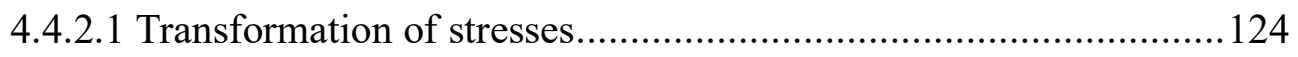

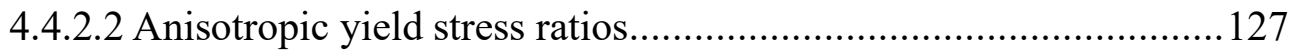




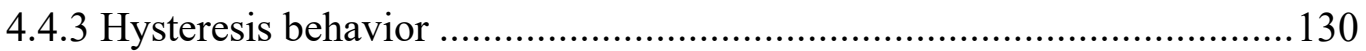

4.4.3.1 Kinematic hardening parameters for LCF simulation ..................130

4.4.3.2 Kinematic hardening parameters for HCF simulation ................... 134

4.5 Fatigue Nucleation Life Prediction of Haynes 282 …………………............135

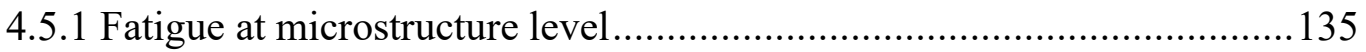

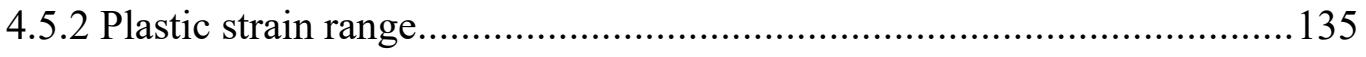

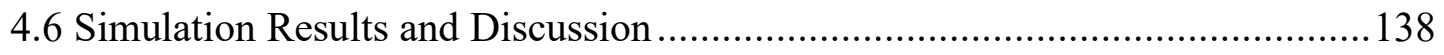

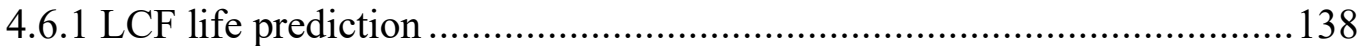

4.6.1.1 Localized plasticity and stress under LCF ................................... 138

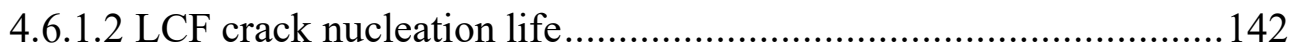

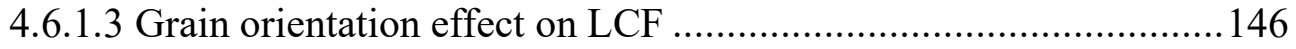

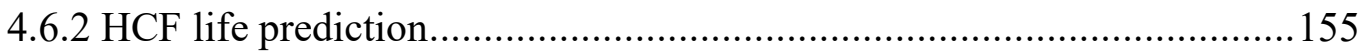

4.6.2.1 Localized plasticity and stress under HCF .................................. 155

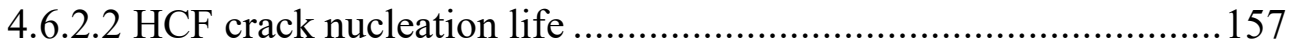

4.6.2.3 Grain orientation effect on HCF .............................................. 160

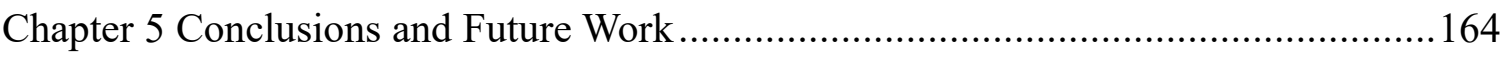

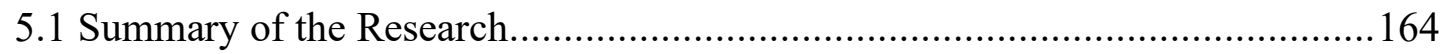

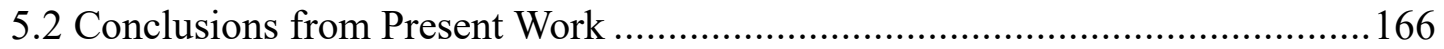

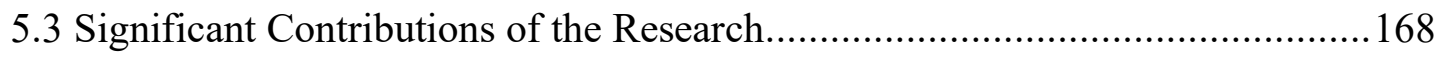

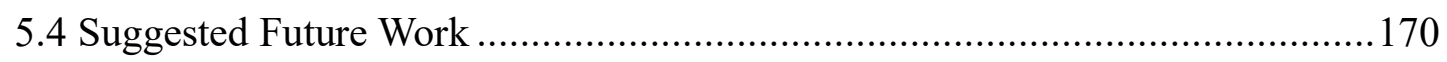

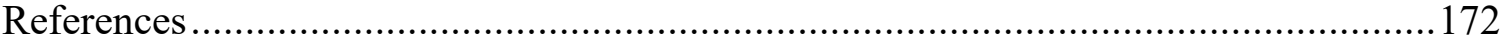

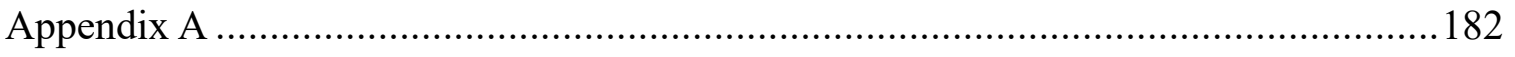

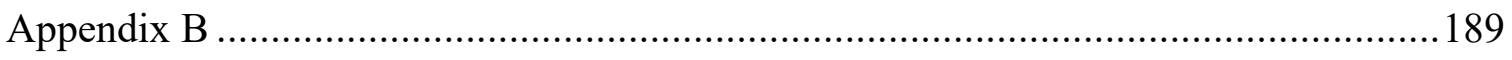

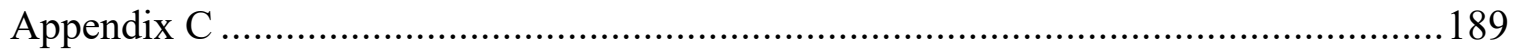

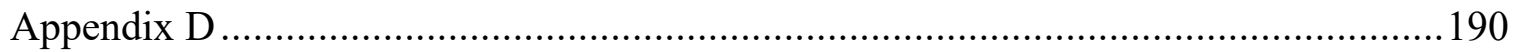




\section{List of Tables}

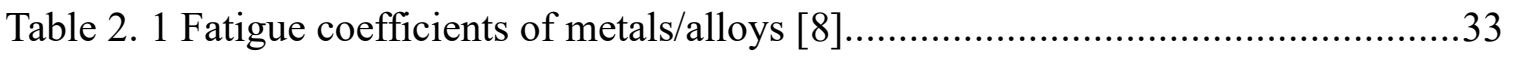

Table 2. 2 Effects of alloying elements in nickel-based superalloys [79] ......................56

Table 2. 3 Tensile properties of nickel-based superalloys [80] [76] ............................57

Table 2. 4 Physical properties of nickel-based superalloys [80] [76] ...........................58

Table 2. 5 Nominal composition (wt.\%) of Haynes 282 alloy [79] ...........................61

Table 3. 1 Chemical compositions of selected materials [91] .................................69

Table 3. 2 Monotonic mechanical properties of selected materials [87] [88] [89] [90] [85]

Table 3. 3 Cyclic properties of selected materials [87] [88] [89] [90] [85] ....................70

Table 3. 4 Calculated fatigue coefficients for selected materials ................................74

Table 3. 5 Monotonic mechanical properties of materials for surface roughness study [19] $[20]$

Table 3. 6 Fatigue coefficients of the TMW model for the materials used in surface roughness analysis

Table 3. 7 Analytical roughness factor $R_{s}$ and lattice resistance $\sigma_{0}$ for aluminum alloy 100 Table 3. 8 Analytical roughness factor $R_{s}$ and lattice resistance $\sigma_{0}$ medium-carbon steel

Table 3. 9 Experimental fatigue life data in different roughness conditions for aluminum

alloy (stress amplitude of $344 \mathrm{MPa}$ ). 103

Table 3. 10 Experimental fatigue life data in different roughness conditions for medium-

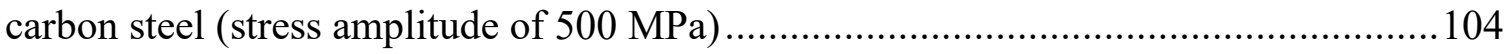

Table 3. 11 Relative values used for determination of $\lambda$ for aluminum alloy ................ 105

Table 3. 12 Relative values used for determination of $\lambda$ for medium-carbon steel......... 105

Table 4. 1 The yielding strength of PWA 1480 in three directions at room temperature [108] 
Table 4. 2 Calculated stress components referring to local coordinate.....

Table 4. 3 Calculated yielding stress ratios and material constants by Hill potential

function

Table 4. 4 Strain hardening parameters for LCF of Haynes 282

Table 4. 5 Strain hardening parameters for HCF of Haynes 282

Table 4. 6 Calculations of LCF life of Haynes 282 for strain range $\Delta \varepsilon=0.84 \%$

Table 4. 7 Calculations of LCF life of Haynes 282 for strain range $\Delta \varepsilon=0.93 \%$

Table 4. 8 Orientations of grains with maximum plastic strain range for LCF simulations

Table 4. 9 Orientations of grains with zero plastic strain range for LCF simulations .... 149

Table 4. 10 Calculations of HCF life of Haynes 282 for stress amplitude $\Delta \sigma / 2=$ $415 \mathrm{MPa}$ 158

Table 4. 11 Calculations of HCF life of Haynes 282 for stress amplitude $\Delta \sigma / 2=$ $400 \mathrm{MPa}$

Table 4. 12 Orientations of grains with maximum plastic strain range for HCF simulations 162 


\section{List of Figures}

Figure 1. 1 Uncontained engine failure of Boeing aircrafts due to fatigue [4] [5]...........2

Figure 2. 1 Transmission electron microscopy (TEM) micrograph of slip bands [8]. ....13

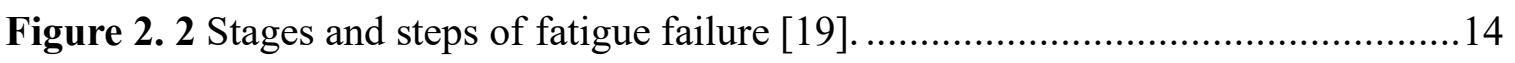

Figure 2. 3 Monotonic and cyclic stress-strain curves....................................... 16

Figure 2. 4 Schematic illustration of the stabilized hysteresis loop for a material...........18

Figure 2. 5 Total strain-life curve composed of Coffin-Manson curve and Basquin curve

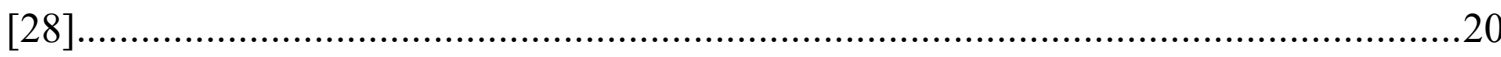

Figure 2. 6 Fatigue crack nucleation from PSBs in aluminum alloy 6063-T6 [111].......21

Figure 2. 7 Schematic illustration of cycle slips leading to crack nucleation [19]..........23

Figure 2. 8 Schematic illustration of dislocation behavior for fatigue crack nucleation:

(a) vacancy dipoles (forming an intrusion), (b) interstitial dipoles (forming an extrusion)

and (c) tripoles (forming an intrusion-extrusion pair) at the surface [8].

Figure 2. 9 Fatigue life of Type 316 stainless steel with different surface finishing. (The symbols represent the referenced experimental data and the lines represent theoretical predictions of Eq (2.28) and Eq (2.30)) [8].

Figure 2. 10 Predicted fatigue curves in comparison with experimental data for various materials [8].

Figure 2. 11 Schematic illustration of (a) isotropic hardening law and (b) kinematic hardening law.

Figure 2. 12 Crystal structures for common metals: (a) FCC structure, (b) BCC structure

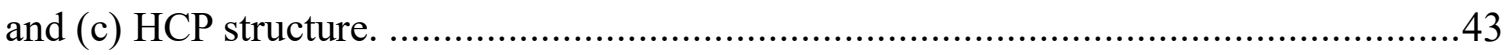

Figure 2. 13 Principle of pole figure plotting [64] ..............................................45

Figure 2. 14 An example of inverse pole figure [65] ........................................46

Figure 2. 15 Decomposition of crystal deformation into elastic and plastic parts [65]...47

Figure 2. 16 Graphical definition of Voronoi tessellation [72] .................................52

Figure 2. 17 A two-dimensional Voronoi diagram. .............................................53

Figure 2. 18 Scanning Electron Microscope (SEM) microstructure of Haynes 282 after standard aging treatment [81]

Figure 3. 1 Comparison between theoretical prediction of Eq (3.1) and Coffin-Manson curve for Al 7075-T6.

Figure 3. 2 Comparison between theoretical prediction of $\mathrm{Eq}$ (3.2) and Basquin curve for Al 7075-T6. 
Figure 3. 3 Comparison between combined theoretical prediction of Eq (3.1) and Eq (3.2) and combined Coffin-Manson-Basquin curve for Al 7075-T6.

Figure 3. 4 Comparison between theoretical prediction of Eq (3.1) and Coffin-Manson curve for Al 2024-T3.

Figure 3. 5 Comparison between theoretical prediction of $\mathrm{Eq}$ (3.2) and Basquin curve for A1 2024-T3. 80

Figure 3. 6 Comparison between combined theoretical prediction of equation (3.1) and (3.2) and combined Coffin-Manson-Basquin curve for Al 2024-T3.

Figure 3. 7 Comparison between theoretical prediction of $\mathrm{Eq}$ (3.1) and Coffin-Manson curve for SAE 1020.

Figure 3. 8 Comparison between theoretical prediction of Eq (3.2) and Basquin curve for SAE 1020.

Figure 3. 9 Comparison between combined theoretical prediction of Eq (3.1) and Eq (3.2) and combined Coffin-Manson-Basquin curve for SAE 1020.

Figure 3. 10 Comparison between theoretical prediction of Eq (3.1) and Coffin-Manson curve for SAE 4340.

Figure 3. 11 Comparison between theoretical prediction of $\mathrm{Eq}$ (3.2) and Basquin curve for SAE 4340.

Figure 3. 12 Comparison between combined theoretical prediction of $\mathrm{Eq}(3.1)$ and $\mathrm{Eq}$ (3.2) and combined Coffin-Manson-Basquin curve for SAE 4340.

Figure 3. 13 Comparison between theoretical prediction of Eq (3.1) and Coffin-Manson curve for Ti-6Al-4V.

Figure 3. 14 Comparison between theoretical prediction of Eq (3.2) and Basquin curve for Ti-6Al-4V.

Figure 3. 15 Comparison between combined theoretical prediction of $\mathrm{Eq}$ (3.1) and $\mathrm{Eq}$ (3.2) and combined Coffin-Manson-Basquin curve for Ti-6Al-4V.

Figure 3. 16 Comparison between theoretical prediction of Eq (3.1) and Coffin-Manson curve for Inconel 617.

Figure 3. 17 Comparison between theoretical prediction of Eq (3.2) and Basquin curve for Inconel 617.

Figure 3. 18 Comparison between combined theoretical prediction of $\mathrm{Eq}(3.1)$ and $\mathrm{Eq}$ (3.2) and combined Coffin-Manson-Basquin curve for Inconel 617.

Figure 3. 19 Comparison between theoretical prediction of Eq (3.1) and Coffin-Manson curve for Inconel 718.

Figure 3. 20 Comparison between theoretical prediction of Eq (3.2) and Basquin curve for Inconel 718. 
Figure 3. 21 Comparison between combined theoretical prediction of Eq (3.1) and Eq (3.2) and combined Coffin-Manson-Basquin curve for Inconel 718.

Figure 3. 22 Comparison between theoretical prediction of Eq (3.1) and Coffin-Manson curve for Haynes 282.

Figure 3. 23 Comparison between theoretical prediction of $\mathrm{Eq} \mathrm{(3.2)} \mathrm{and} \mathrm{Basquin} \mathrm{curve}$ for Inconel Haynes 282 .

Figure 3. 24 Comparison between combined theoretical prediction of $\mathrm{Eq}(3.1)$ and $\mathrm{Eq}$ (3.2) and combined Coffin-Manson-Basquin curve for Haynes 282.

Figure 3. 25 Experimental fatigue life of aluminum alloy with different arithmetical mean deviation of the assessed profile $R_{a}$ values against stress amplitude [103]

Figure 3. 26 Experimental fatigue life of medium-carbon steel with different arithmetical mean deviation of the assessed profile $R_{a}$ values against stress amplitude [102]. .98

Figure 3. 27 Comparison of experimental fatigue life with analytical fatigue life by Eq (3.2) for aluminum alloy with different values of $R_{a}$.

Figure 3. 28 Comparison of experimental fatigue life with analytical fatigue life by Eq (3.2) for of medium-carbon steel with different values of $R_{a}$. 100

Figure 3. 29 Relationship between roughness amplitude parameter $R_{a}$ and roughness factor $\mathrm{R}_{\mathrm{S}}$ for aluminum alloy and medium-carbon steel.

Figure 3. 30 Relationship between roughness amplitude parameter $R_{a}$ and lattice resistance $\sigma_{0}$ for aluminum alloy and medium-carbon steel.

Figure 3. 31 Determination of exponential factor $\lambda$ for aluminum alloy and mediumcarbon steel.

Figure 4. 1 SEM image of fatigue cracks in Haynes 282 microstructure for LCF simulation [84].

Figure 4. 2 Stabilized hysteresis loops of Haynes 282 under LCF at room temperature $[84]$

Figure 4. 3 Optical image of Haynes 282 microstructure for HCF simulation [86] ..... 112

Figure 4. 4 Illustration of RVE for Haynes 282 ..................................................... 114

Figure 4. 5 3D RVE model of Haynes 282 for microstructure FEA simulation............ 116

Figure 4. 6 Definition of grain orientation........................................................ 117

Figure 4. 7 Illustration of crystal frame rotation about (a) $z$ axis (first), (b) rotated $x$ axis (second) and (c) rotated $z$ axis (third).

Figure 4. 8 Illustration of boundary conditions of the RVE model........................... 120

Figure 4. 9 Convergence study with four different meshes. .................................... 122

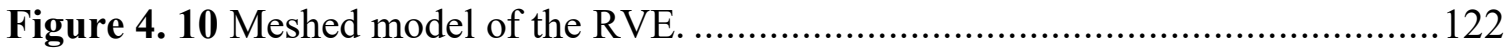

Figure 4. 11 Definitions of two coordinate systems. ......................................... 125 
Figure 4. 12 Stabilized hysteresis loops of Haynes 282 at strain range of $0.84 \%$. 132

Figure 4. 13 Stabilized hysteresis loops of Haynes 282 at strain range of $0.93 \%$. 132

Figure 4. 14 Stabilized hysteresis loops of Haynes 282 at strain range of $1.34 \%$. 133

Figure 4. 15 Stabilized hysteresis loops of Haynes 282 at strain range of $1.70 \%$

Figure 4. 16 Tensile curves for HCF of Haynes 282.

Figure 4. 17 Stabilization of plastic strain magnitude with the number of iteration increased.

Figure 4. 18 Contour plot of plastic strain magnitude (PEMAG) at the maximum strain level of 4 th cycle for $\mathrm{LCF}_{-\mathrm{T}} \mathrm{T}_{14}$ (total strain range $\Delta \varepsilon=0.84 \%$ ).

Figure 4. 19 Contour plot of Von Mises stress (S) at the maximum strain level of 4th cycle for $\mathrm{LCF}_{-\mathrm{T}} \mathrm{T}_{14}$ (total strain range $\Delta \varepsilon=0.84 \%$ ).

Figure 4. 20 Distribution of localized plastic flow at free surface $(z=0)$ for LCF-T 14 (total strain range $\Delta \varepsilon=0.84 \%$ ).

Figure 4. 21 Demonstration of locations of large and small plastic regions.

Figure 4. 22 Element with the maximum plastic strain magnitude at the maximum strain of loading for $\mathrm{LCF}_{-} \mathrm{T}_{6}$ (total strain range $\Delta \varepsilon=0.84 \%$ ).

Figure 4. 23 Element with the maximum plastic strain magnitude at the maximum strain of reverse loading for $\mathrm{LCF}_{-} \mathrm{T}_{6}$ (total strain range $\Delta \varepsilon=0.84 \%$ ).

Figure 4. 24 Comparison between calculated LCF crack nucleation life and experimental total-life for stain ranges of $84 \%$ and $93 \%$.

Figure 4. 25 Inverse pole figure of grains with maximum plastic strain range for all LCF simulations.

Figure 4. 26 Contour plots of orientations of grains with maximum plastic strain range for all LCF simulations.

Figure 4. 27 Inverse pole figure for grains with zero plastic strain range for all LCF simulations.

Figure 4. 28 Contour plots of orientations of grains with zero plastic strain range for all LCF simulations.

Figure 4. 29 Demonstration of adjacent grains in large plastic region at grain boundary.

Figure 4. 30 Inverse pole figure of adjacent grains in large plastic region at grain boundary.

Figure 4. 31 Contour plots of plastic strain magnitude (PEMAG) at maximum strain level of 4 th cycle for $\mathrm{HCF}_{4}$ (stress amplitude $\Delta \sigma / 2=400 \mathrm{MPa}$ ). 156

Figure 4. 32 Contour plots of Von Mises stress (S) at maximum strain level of 4th cycle for $\mathrm{HCF}_{-} \mathrm{T}_{4}$ (stress amplitude $\Delta \sigma / 2=400 \mathrm{MPa}$ ). 
Figure 4. 33 Element with maximum plastic strain magnitude at maximum strain for $\mathrm{HCF}_{-} \mathrm{T}_{4}$ (stress amplitude $\Delta \sigma / 2=400 \mathrm{MPa}$ ).

Figure 4. 34 Comparison between calculated HCF crack nucleation life and experimental total life for stress amplitudes of $415 \mathrm{MPa}$ and $400 \mathrm{MPa}$. 160

Figure 4. 35 Inverse pole figure of grains with maximum plastic strain range for all HCF simulations.

Figure 4. 36 Contour plots of orientations of grains with maximum plastic strain range for all HCF simulations.. 


\section{Acronyms}

\begin{tabular}{|c|c|}
\hline PSB & Persistent Slip Band \\
\hline TEM & Transmission Electron Microscopy \\
\hline USAF & United State Air Force \\
\hline USN & Unite State Navy \\
\hline LEFM & Linear Elastic Fracture Mechanics \\
\hline $\mathbf{L C F}$ & Low Cycle Fatigue \\
\hline HCF & High Cycle Fatigue \\
\hline $\mathbf{C G}$ & Coarse-Grained \\
\hline FG & Fine-Grained \\
\hline ASTM & American Society for Testing of Materials \\
\hline OEM & Original Equipment Manufacturer \\
\hline FEM & Finite Element Method \\
\hline 2D & Two-Dimensional \\
\hline FCC & Face-Centered Cubic \\
\hline BCC & Body-Centered Cubic \\
\hline HCP & Hexagonal Closest Packed \\
\hline RVE & Representative Volume Element \\
\hline A-USC & Advanced Ultra-Supercritical \\
\hline SAE & Scanning Electron Microscope \\
\hline TMW & Tanaka-Mura-Wu \\
\hline SAE & Society of Automotive Engineers \\
\hline $\mathbf{P N}$ & Peierls-Nabarro \\
\hline FEA & Finite Element Analysis \\
\hline ISO & International Organization for Standardization \\
\hline 3D & Three-Dimensional \\
\hline CAE & Computer-Aided Engineering \\
\hline EBSD & Electron Backscatter Diffraction \\
\hline PEMAG & Plastic Strain Magnitude \\
\hline
\end{tabular}




\section{Nomenclature}

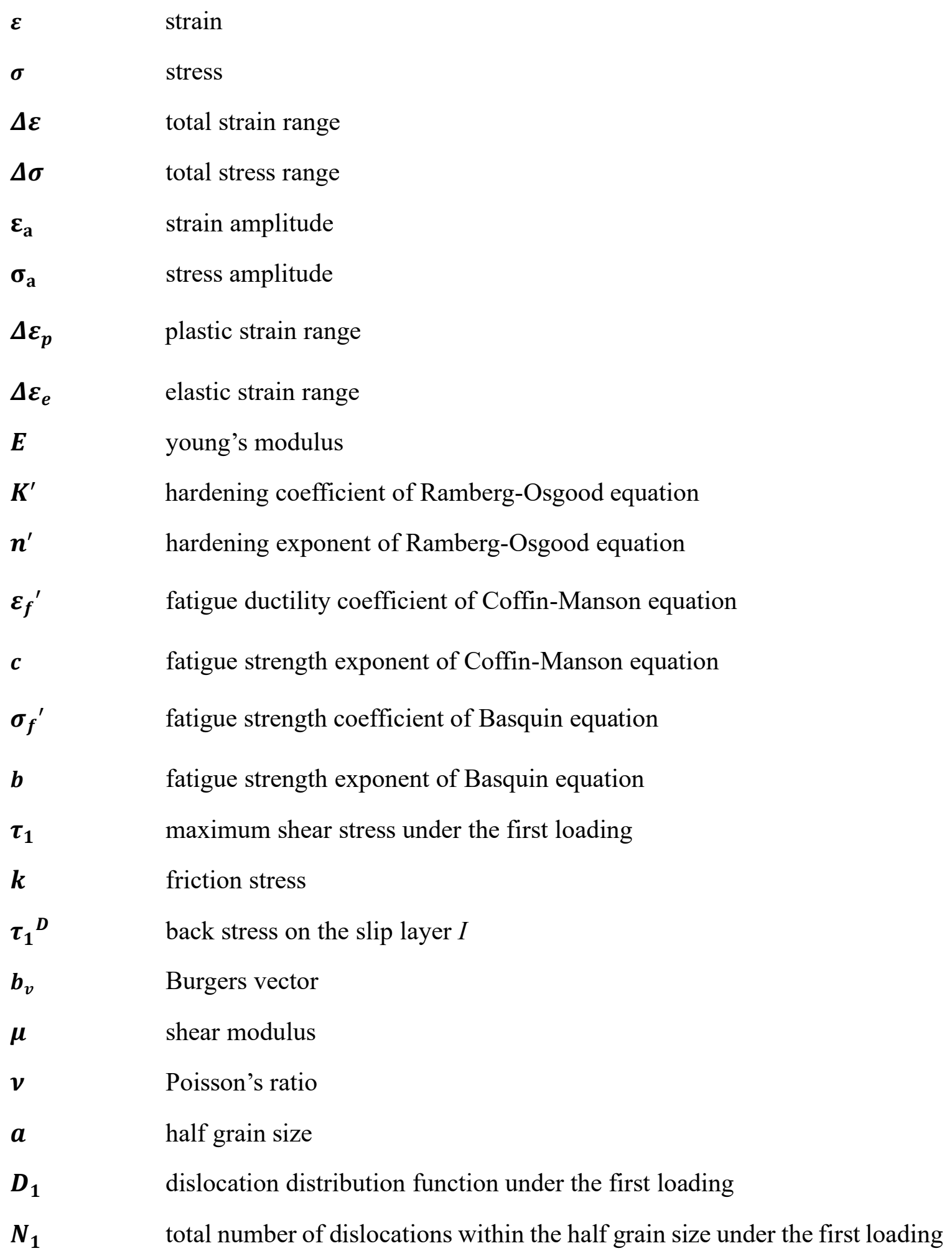




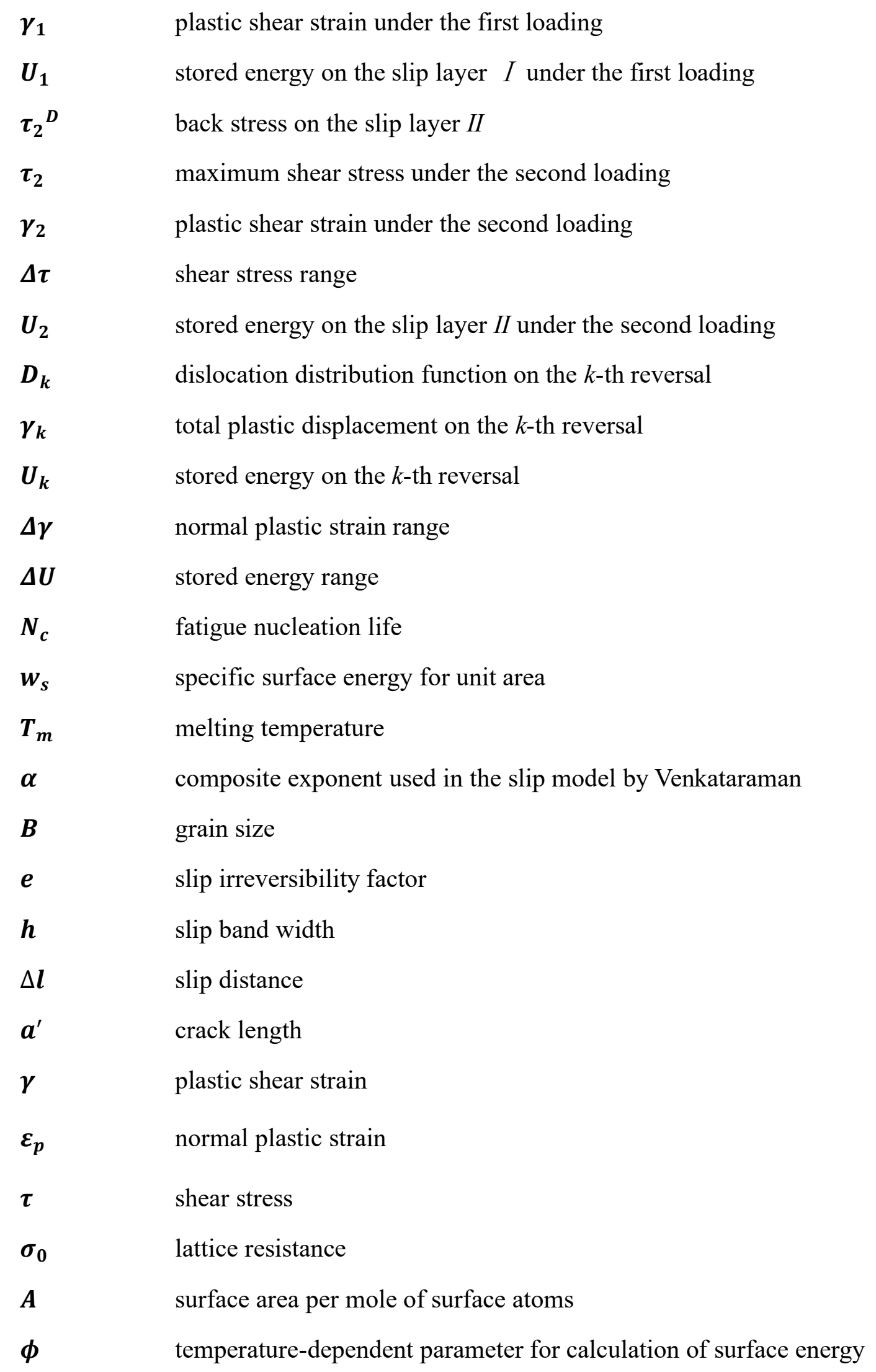




\begin{tabular}{|c|c|}
\hline $\boldsymbol{R}$ & Boltzmann constant \\
\hline$\sigma_{i j}$ & stress component \\
\hline$\varepsilon_{k l}$ & strain component \\
\hline$C_{m n}$ & material elastic constant \\
\hline$\sigma_{1} \sigma_{2} \sigma_{3}$ & principal stresses \\
\hline$\sigma_{s}$ & yield strength of material \\
\hline$f$ & yield function in the Cartesian system \\
\hline$F G H L M N$ & material constants defined in Hill potential function \\
\hline$f_{0}$ & yield function \\
\hline $\boldsymbol{K}$ & hardening parameters \\
\hline$K_{0}$ & initial yield surface size \\
\hline$\alpha_{i j}$ & back stress \\
\hline$e_{1} e_{2} e_{3}$ & basic vector of unit cell \\
\hline $\boldsymbol{h} \boldsymbol{k} \boldsymbol{l}$ & Miller indices \\
\hline $\boldsymbol{F}$ & deformation gradient \\
\hline$X_{j}$ & reference particle position \\
\hline$x_{i}$ & current particle position \\
\hline$F^{p}$ & plastic deformation gradient \\
\hline$F^{e}$ & elastic deformation gradient \\
\hline $\boldsymbol{L}$ & velocity gradient \\
\hline$\dot{\gamma}^{\alpha}$ & shear rate in the $\alpha-$ th activated slip system \\
\hline$L^{p}$ & plastic velocity gradient \\
\hline$s^{\alpha}$ & slip direction of $\alpha$-th activated slip system \\
\hline$m^{\alpha}$ & normal to the slip plane of $\alpha-$ th activated slip system \\
\hline$\tau^{\alpha}$ & resolved shear stress of $\alpha$-th activated slip system \\
\hline $\boldsymbol{D}$ & stretching tensor \\
\hline $\boldsymbol{W}$ & spin tensor \\
\hline
\end{tabular}




\begin{tabular}{|c|c|}
\hline$D^{e}$ & elastic stretching tensor \\
\hline$D^{p}$ & plastic stretching tensor \\
\hline$w^{e}$ & elastic spin tensor \\
\hline$W^{p}$ & plastic spin tensor \\
\hline$\dot{\gamma}^{\alpha}$ & slip rate on the $\alpha$-th activated slip system \\
\hline$n$ & power-law exponent \\
\hline$g^{\alpha}$ & evolution of strain hardening for isotropic hardening \\
\hline$\chi^{\alpha}$ & evolution of strain hardening for kinematic hardening \\
\hline$r p q$ & material constants \\
\hline$h_{\alpha \alpha}$ & self-hardening rate \\
\hline$h_{\alpha \beta}$ & latent-hardening rate \\
\hline$h_{0}$ & initial hardening modulus \\
\hline$\tau_{s}$ & breakthrough stress \\
\hline$\tau_{0}$ & yield stress \\
\hline$\tau_{s}$ & breakthrough stress \\
\hline$\gamma_{a}$ & accumulated shear strain \\
\hline$D^{\prime}$ & scale of bulk material \\
\hline$L^{\prime}$ & scale of representative volume of element \\
\hline$d^{\prime}$ & microstructure scale inside of representative volume of element \\
\hline$z_{i}$ & Voronoi point \\
\hline$V_{i}$ & Voronoi tessellation \\
\hline $\boldsymbol{P}_{i}$ & Voronoi generator \\
\hline$e$ & edge length of unit cell \\
\hline$\sigma_{y s}$ & yield stress \\
\hline$\sigma_{u}$ & tensile strength \\
\hline$E I$ & elongation \\
\hline$N_{f}$ & total fatigue life \\
\hline
\end{tabular}




\begin{tabular}{|c|c|}
\hline $\boldsymbol{R}_{a}$ & arithmetical mean deviation of roughness profile \\
\hline$R_{q}$ & root mean squared of roughness profile \\
\hline$R_{p}$ & maximum peak height of roughness profile \\
\hline \multirow[t]{2}{*}{$\lambda$} & exponent for the fitted exponential relation between fatigue life and surface \\
\hline & roughness parameter $R_{a}$ \\
\hline$\varphi \theta \psi$ & Bunge Euler angles \\
\hline$t$ & traction applied to the constrained plane of representative volume element \\
\hline$d$ & displacement applied to the loading plane of representative volume element \\
\hline$d_{R}$ & edge length of representative volume element \\
\hline$[\sigma]$ & stress vector in the global coordinate system \\
\hline$\left[\sigma^{\prime}\right]$ & stress vector in the local coordinate system \\
\hline$[Q]$ & relation matrix in coordinate transformation \\
\hline$\alpha \beta \gamma$ & direction cosines between the axes in two coordinate systems \\
\hline $\boldsymbol{R}_{i j}$ & Hill yield stress ratio \\
\hline $\bar{\sigma}_{i j}$ & measured yield stress \\
\hline$\sigma^{o}$ & reference yield stress \\
\hline$\tau^{o}$ & reference shear stress \\
\hline$\varepsilon^{p}$ & plastic strain magnitude defined in Abaqus package \\
\hline$\varepsilon^{p l}$ & plastic strain tensor \\
\hline$\varepsilon_{i j}^{p}$ & normal plastic strain component \\
\hline$\gamma_{i j}^{p}$ & shear plastic strain component \\
\hline$\varepsilon_{t}^{p}$ & plastic strain magnitude at the maximum strain amplitude during tension \\
\hline$\varepsilon_{c}^{p}$ & $\begin{array}{l}\text { plastic strain magnitude at the maximum strain amplitude during } \\
\text { compression }\end{array}$ \\
\hline
\end{tabular}




\section{Chapter 1 Introduction}

\subsection{Background of the Research}

Fatigue contributes to at least 90 percent of all service failures of components subjected to repeated loading and vibration, as experienced by automobiles, aircraft, compressors, pumps, turbines, etc. [1]. This type of failure is extremely harmful for the structural integrity of engineering components because fatigue crack nucleation is very difficult to predict as it can be affected by many uncertain factors including manufacturing defects, microstructure and roughness, under given loading conditions and environments. Particularly for the gas turbine engines which are widely used in the aircraft and power-generation industry, the components such as low-pressure compressor blades are often subjected to synergetic effects of high- and low-cycle loads in operation. In addition, they can be affected by oxidation due to high service temperature. Such harsh working condition makes the structures of gas turbine engines susceptible to fatigue damage [2]. Fatigue crack nucleation and propagation can affect the fatigue life and even directly lead to failure of gas turbine engine components, which can cause tremendous economic loss [3]. Uncontained engine failure of Boeing aircrafts due to fatigue is shown as an example in Figure 1.1. Therefore, fatigue design to improve durability of engineering components has been a continuing effort ever since August Wholer found fatigue cracks in an axle of failed locomotive in Versailes, France, 1842. 


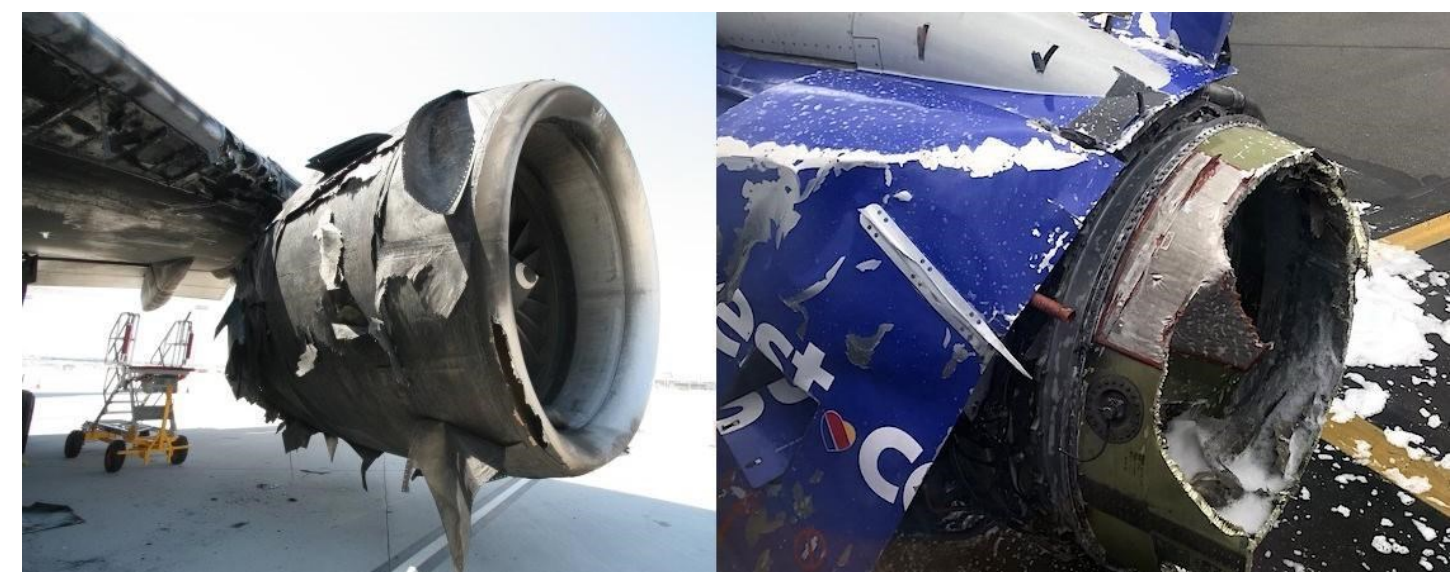

Figure 1. 1 Uncontained engine failure of Boeing aircrafts due to fatigue [4] [5].

Fatigue life prediction is one of the most important concerns for fatigue design. Traditionally, engineers and researchers have to perform fatigue testing based on ASTM standard [6] to evaluate a material's fatigue life, which results in high costs in time and money, thus prolonging the development cycle of material and component design for mechanical/structural systems. Although several fatigue life models such as CoffinManson equation and Basquin equation [7] have been developed to speed up the fatigue lifetime assessment, they still highly rely on the fitting of testing data, which do not essentially solve the high cost issue, especially for the development of new materials. Recently, $\mathrm{Wu}$ [8] [9] proposed an analytical model (the Tanaka-Mura-Wu model or the TMW model) which relates fatigue crack nucleation life to purely physical properties and surface finish of materials. Although this model does not include all possible factors affecting material's fatigue behavior (loading spectrum, residual stress, etc.), it has been applied on a few pure metals such as iron and copper and shown good agreement with 
experimental observations.

In addition to the fatigue life prediction, the assessment of fatigue life scattering is also a crucial consideration in fatigue design. Commonly, the scattering in fatigue life can be observed from a factor of one to over several orders of magnitude for a given test or service condition [10]. The variability of inhomogeneities in the microstructure including grain size and grain orientation can be partially accountable for fatigue life scattering appearing on the components made of polycrystalline materials. These inhomogeneities contribute to the development of local stress and strain field in the microstructure, which controls fatigue crack nucleation [11]. However, the stochastic nature of microstructure influence on fatigue life scattering makes itself very hard to identify by fatigue testing, because even for the same material, microstructure can vary from different fabrication methods and heat treatment processes. Hence, a large number of fatigue coupons may be required to obtain reliable data that cover all extremes. Moreover, empirical fatigue life prediction models often cannot directly relate fatigue life to the variability in microstructure. Nowadays, owing to the development of computing technology, numerical modeling has been introduced to replace the physical testing to evaluate the existing fatigue durability design and also to further improve it, which is known as "certification by analysis". This concept has been successfully utilized in aviation research and it potentially can save both development time and cost [12]. 


\subsection{Objectives of the Research}

As indicated above, conventional approach using physical testing for fatigue durability design of engineering components has its limitations due to low efficiency and high cost. The combination of effective fatigue life prediction model and appropriate numerical simulation can work as an alternative approach in prognosis and health management of existing or newly designed mechanical systems, with accurate life prediction, offering tremendous savings in life cycle management.

Therefore, the present research is conducted aiming at the following objectives:

1. Assess the effectiveness and applicability of the TMW model over the entire fatigue regime from low cycle fatigue (LCF) to high cycle fatigue (HCF) for structural materials.

2. Predict the fatigue crack nucleation lives of eight different structural materials using the TMW model thus further assess the effectiveness of the TMW model for a wide range of structural materials.

3. Improve the TMW model for practicability by replacing the surface roughness factor in the TMW model in terms of the arithmetical mean deviation of the assessed profile which is experimentally obtainable.

4. Investigate the prediction scattering of fatigue crack nucleation life via modelling of the microstructural inhomogeneity (grain orientation) of nickel-based alloy Haynes 282 with 
finite element analysis (FEA).

5. Develop the approach that combines the TMW model with the FEA modelling of microstructural inhomogeneity (grain orientation) for the fatigue life prediction and its scattering of Haynes 282 so as to establish a microstructural computational fatigue design approach for other structural materials.

\subsection{Tasks and Methodologies}

To achieve the proposed objectives, the entire research project is implemented by completing the tasks in three parts, as detailed below:

Part 1-Fatigue life prediction with the TMW model

(1) Eight different structural materials including aluminum alloys 7075-T6 and 2024-T3, alloy steels SAE 1020 and SAE 4340, titanium alloy Ti-6Al-4V, and nickel-based alloys Inconel 617, Inconel 718 and Haynes 282 are selected, which represent a wide span of materials for the structures and components under fatigue loading in service and also have the fatigue testing data available in literature for comparison

(2) The material properties and parameters that the TMW model requires are determined for the selected alloys and steels either from the data reported in literature or from calculations. 
(3) The fatigue curves of LCF region and HCF region are developed for the selected materials based on the strain-based formula and the stress-based formula of the TMW model, respectively.

(4) The fatigue curves of LCF region and HCF region are developed for the selected materials according to the Coffin-Manson equation and the Basquin equation, respectively, which are all derived from experimental data fitting.

(5) The predicted fatigue crack nucleation lives of the selected materials from the TMW model are compared with the calculated results from the Coffin-Manson equation and the Basquin equation for both LCF region and HCF region, thus the effectiveness of the TMW model is further analyzed.

\section{Task 2 - Improvement of the TMW model in surface roughness factor}

(1) The mathematic expression describing the correlation between the surface roughness factor in the strain-based formula of the TMW model for LCF and the profile roughness parameter which can be determined experimentally is developed based on the fatigue testing data of aluminum alloy and medium-carbon steel reported in literature by regression analysis.

(2) The mathematic expression describing the correlation between the surface roughness factor in the stress-based formula of the TMW model for HCF and the profile roughness 
parameter which can be determined experimentally is developed based on the fatigue testing data and lattice resistance of aluminum alloy and medium-carbon steel reported in literature by regression analysis.

(3) The TMW model is revised with the profile roughness parameter substituting for the surface roughness factor and the difference of surface roughness influence on the LCF crack nucleation life and the HCF crack nucleation life of structural materials is analyzed.

Task 3 - FEA simulation of microstructural inhomogeneity

(1) A 3D microstructure model is created for FEA simulation of the microstructural inhomogeneity of Haynes 282, which represents the material representative volume elements (RVE) with twenty random grain orientation distribution functions generated for the LCF simulation and for the HCF simulation, respectively, using Voronoi tessellation method and Python Script computer program.

(2) The material parameters and constants of Haynes 282 for the microstructure FEA model including anisotropic elastic constants, anisotropic yield stress ratios, strain hardening parameters are calculated based on the macro material properties of Haynes 282 and equivalent alloy.

(3) The microstructure RVE model is meshed and applied with boundary conditions to determine the stress and strain profiles under the strain ranges of $0.84 \%$ and $0.93 \%$ for LCF 
and stress amplitudes of $400 \mathrm{MPa}$ and $415 \mathrm{MPa}$ for HCF using the commercial FEM Abaqus package.

(4) The plastic strains developed in the microstructure of Haynes 282 with different grain orientation distributions are analyzed and the maximum value of plastic strain is identified for each grain orientation distribution in both LCF and HCF simulations at different strain and stress levels, respectively.

(5) The LCF and HCF crack nucleation lives of Haynes 282 are predicted using the TMW model with the maximum plastic strain range value obtained from the FEA simulation for each grain orientation distribution and the fatigue life prediction scattering then can be determined.

(6) The predicted crack nucleation lives of Haynes 282 from the combined approach of the TMW model and the FEA simulation with microstructural inhomogeneity are compared with the experimental data reported in literature thus to assess this approach for effectiveness.

(7) The virtual inverse pole figures are developed through Matlab codes to display the orientations of grains where crack nucleation occurs, in order to study the effect of grain orientations on the fatigue crack nucleation life of Haynes 282 alloy. 


\subsection{Thesis Organization}

The entire research work presented in this thesis can be covered by five chapters, as detailed below.

Chapter $\mathbf{1}$ is an introduction to this research, indicating the motivation and objectives of this research, the tasks that have been completed and the methodologies that are used in this research, as well as the outline of this thesis.

Chapter 2 gives extensive literature review on the research topic. It starts from the overview of fatigue concepts and prediction of fatigue crack nucleation life to fundamental aspects of microstructure modeling for fatigue studies including theory of anisotropic elasticity, yielding criteria and hardening law, etc. Other contents covered in this chapter include the properties and applications of various nickel-based superalloys, in particular, the details of Haynes 282 alloy, which is the main material selected in this study.

Chapter 3 provides the detailed procedures of the fatigue crack nucleation life prediction for eight different alloys and steels using the TMW model, including the material selection, the determination of material properties and model parameters, the development of fatigue curves for both LCF and HCF, as well as the comparison of the predicted fatigue curves from the TMW model with the fatigue data or fatigue curves obtained from the CoffinManson and Basquin relations. The improvement of the TMW model by using an experimentally obtainable surface roughness parameter and the analysis of surface 
roughness effect on the fatigue crack nucleation life of structural materials are also described in this chapter.

Chapter 4 presents all aspects of the microstructure-based FEA simulation for Haynes 282 including the creation RVE, the determination of material properties and constants for the FEA model, the result analyses of plastic strains with respect to inhomogeneity (grain orientation). The fatigue life prediction of Haynes 282 with the combination of the TMW model and the microstructural FEA simulation is also presented in this chapter, along with the comparison with the experimental data given in literature.

Chapter 5 summarizes this research and presents the conclusions drawn from this research. The significant contributions of this research are highlighted and the future work for this project is also proposed in this chapter. 


\section{Chapter 2 Literature Review}

\subsection{Fatigue of Materials}

Generally, mechanical failure refers to the breakdown of a machine component while operating under its design constraints. This failure can be attributed to any change in shape, size or material properties of the machine component. Fatigue is one of the mechanical failure modes occurring under cyclic loading. Usually, the synergetic effect of time/cycle, load and temperature is involved in the fatigue phenomenon [13]. In this thesis, the fatigue performance of selected eight different alloys and steels, in particular, the nickel-based superalloy Haynes 282, are studied through a microstructural analysis approach.

\subsubsection{Overview of fatigue}

Fatigue of materials has been studied for more than 150 years since August Wöhler first found fatigue cracks in the axle of a crashed locomotive in the 1840s. It has been a constant subject of intensive study because it impacts on the structural life of many components and even safety of the people around [14]. The fatigue damage refers to a process of permanent structural change occurring in a material that arises from repeated application of stress and strain [15]. Fatigue damage accumulation can be affected by many factors such as applied load profile (the maximum and minimum load, load range, frequency), residual stress, microstructure, surface finish, temperature, environment and so on [16]. It accounts for almost $90 \%$ in-service component failures [8]. Hence, it is essential to thoroughly 
understand the mechanism of fatigue damage accumulation and the controlling factors in material/structural design and application.

It has been generally understood that fatigue damage accumulates with plastic deformation during cyclic loading. In most polycrystalline materials, the primary mechanism of plastic deformation is crystallographic slip via dislocation motion. During cyclic deformation, dislocations multiply and accumulate within materials. These dislocations begin to move when shear stress within one or more grains reaches the critical resolved shear stress on particular slip systems. As slip continues, dislocation pile-ups are generated by the impedance of solute atoms, precipitates or grain boundaries. After that, local stress leads to the nucleation of blocked dislocations to replace the obstacle through climb, jog, kink, cross-slip or shearing process. Upon subsequent cycles of loading, dislocations move in the same pathways because these pathways are associated with low energy. Plastic deformation also results in positive and negative dislocations, which are annihilated by cross-slip of screw dislocations and climb of edge dislocations. The annihilation of dislocations gives rise to the formation of vacancies. On the other hand, dislocation multiplication from Frank-Reed sources causes increase in dislocation density and dislocation interaction, which implies work hardening within the slip band. Consequently, the slip process in the forward loading cannot be fully recovered in the reverse loading. Due to the accumulation of slip irreversibility, eventually dislocations form unique ladder structures with low energy in the form of persistent slip bands (PSBs), which are potential 
crack nucleation sites [17]. A transmission electron microscopy (TEM) micrograph of slip bands resulting in fatigue is shown in Figure 2.1 where PSBs appear on the material surface in the form of lamellar wall structure.

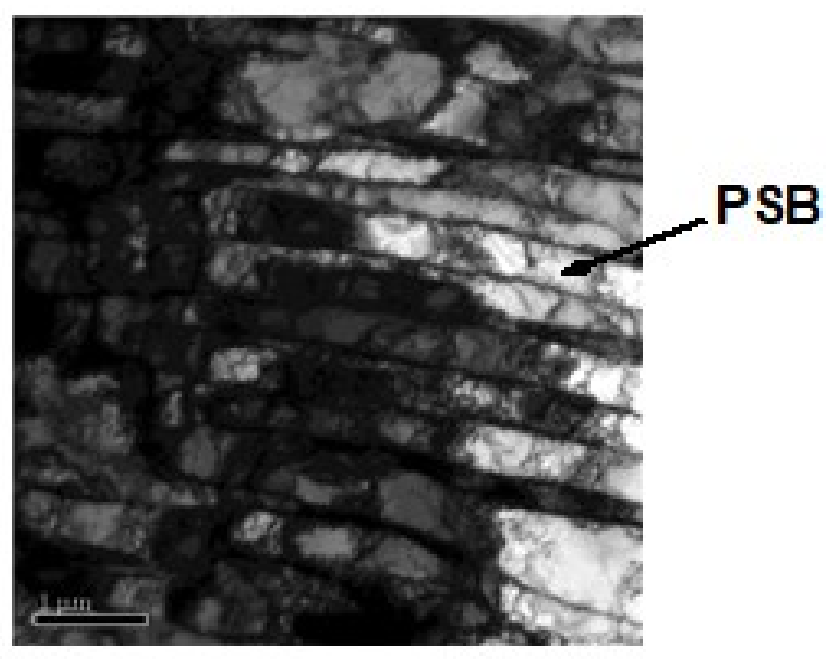

Figure 2. 1 Transmission electron microscopy (TEM) micrograph of slip bands [8].

Generally, the fatigue life of a part can be roughly separated into two stages: crack initiation and crack growth. In the former, some microcracks nucleate in the slip bands of the part and they are invisible for the majority of fatigue life. After crack nucleation, the crack starts to grow from these micro defects. However, crack growth in this stage is at a relatively slow rate due to the effects of microstructural constraint such as grain boundaries. When the microcrack reaches a grain boundary, crack growth can be blocked due to the sudden change in orientation of the slip systems in the adjacent grains [18]. In the latter, the microcracks grow further into a macroscopic size, reaching to a more observable level [19]. 
Finally, fracture takes place when the remaining uncracked cross-section of the part is too weak to sustain the load. The two stages of fatigue life are diagrammed in Figure 2.2.

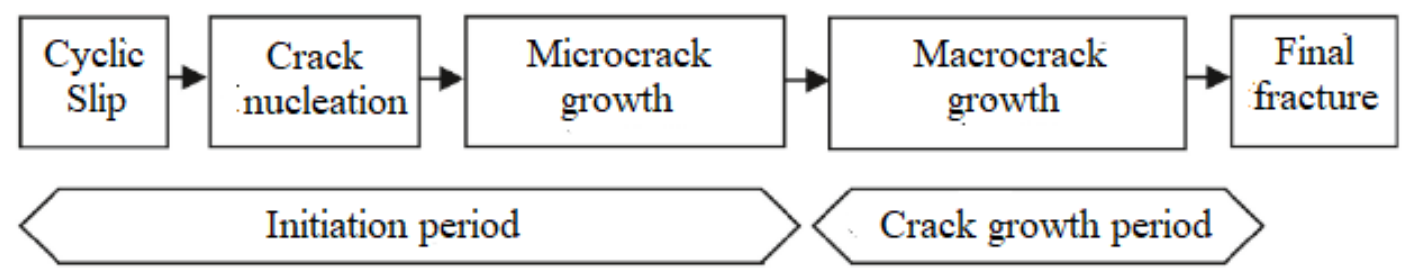

Figure 2. 2 Stages and steps of fatigue failure [19].

In spite of the advanced testing techniques, the transition from crack initiation to crack growth stage has not been clearly identified. Qualitatively, the onset of crack growth stage starts when microcracks can be treated as real cracks in fracture mechanics sense, i.e., with the formation of free crack surfaces that do not transmit stresses. In engineering, the first appearance of a detectable crack size is usually identified as the transition from crack initiation to crack growth, which marks the point from then on fracture mechanics is applied for crack growth analysis. For example, the United State Air Force (USAF) defines the crack nucleation size to be 0.05 in $(1.27 \mathrm{~mm})$ [20]; whereas the Unite State Navy (USN) defines the crack nucleation size to be 0.01 in $(0.254 \mathrm{~mm})$ [21]. In the case of macrocracks, plastic deformation is discernable by experimental techniques and the plastic zone size is usually small compared to the crack size such that linear elastic fracture mechanics (LEFM) can be used to analyze the macrocrack growth kinetics. However, LEFM is not applicable to microcrack growth when the plastic zone is large compared to the microcrack size. The 
large-scale plasticity around a microcrack renders an inelastic behavior of the crack [22]. Therefore, the definition of crack initiation becomes a critical issue to the holistic structural integrity process, as schematically shown in Figure 2.2.

The above critical issue has not been resolved. The arbitrary size definition as aforementioned smears the process of crack nucleation and growth up to the size with an ambiguous life (usually estimated by coupon-based stress-life or strain-life methods). The ambiguity lies in that i) the extent of uniformity of local stress/strain distribution in a structure depends on the local geometrical features such as notches or holes and the load, and ii) the relative size of crack initiation to microstructural characteristic length such as grain size has not been identified. Some analytical definitions have been proposed, mainly via macroscopic approaches [23][24]. To clarify the very early stage of fatigue failure, i.e. crack initiation or crack nucleation, microstructural definition and simulation must be sought, which is the focus of the present study.

Conventionally, fatigue is classified into: i) low-cycle fatigue (LCF) and ii) high-cycle fatigue (HCF) [8]. LCF usually occurs in less than $10^{4}$ cycles with relatively high peak stress exceeding the material's yield strength. Hence, LCF is commonly associated with a substantial amount of cyclic plasticity. On the other hand, HCF, with the number of cycles greater than $10^{4}$, occurs in the macroscopic elastic regime where cyclic stresses are below the yield point of the material [25]. The relationships between the fatigue life and the applied load range, either in strain-control or stress-control tests, established for smooth 
coupons, are often used to define the "crack initiation life", but there is no crack initiation size definition in these tests.

In mechanical fatigue, the material's nonlinear deformation behavior is vitally important for the fatigue resistance. A material may exhibit a monotonic stress-strain relationship during loading of each cycle, but this relationship may change as the material is being cyclically hardened/softened as cycle continues. A cyclic stress-strain curve can be established by connecting the peak stresses of stabilized hysteresis loops cycled at different cyclic strain amplitudes. The comparison between monotonic stress-strain curve and cyclic stress-strain curve is shown in Figure 2.3.

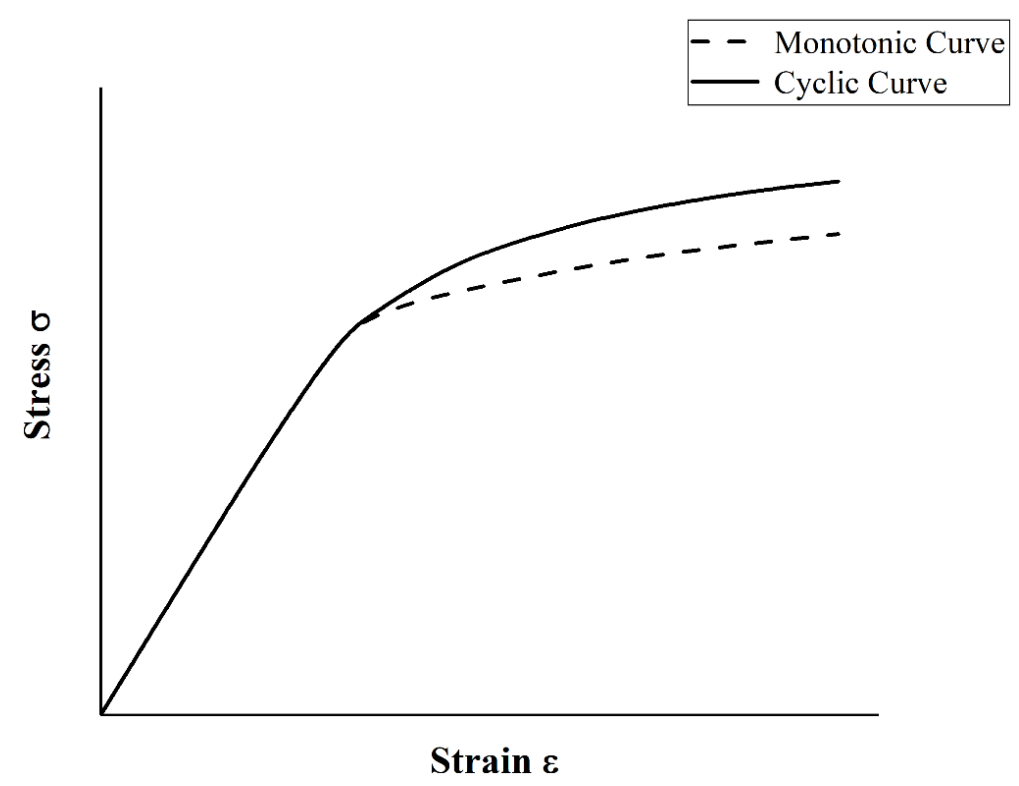

Figure 2. 3 Monotonic and cyclic stress-strain curves. 
A hysteresis loop, as shown in Figure 2.4, reflects the plastic energy absorbed by the material under cyclic loading. The total width of the loop is the total strain range $\Delta \varepsilon$ and the total height of the loop is the total stress range $\Delta \sigma$, while $\varepsilon_{a}$ and $\sigma_{a}$ denote the strain amplitude and stress amplitude, respectively, as defined by:

$$
\begin{aligned}
& \varepsilon_{a}=\frac{\Delta \varepsilon}{2} \\
& \sigma_{a}=\frac{\Delta \sigma}{2}
\end{aligned}
$$

The total strain is the sum of the elastic and plastic strain ranges:

$$
\Delta \varepsilon=\Delta \varepsilon_{e}+\Delta \varepsilon_{p}
$$

where $\Delta \varepsilon_{p}$ and $\Delta \varepsilon_{e}$ are plastic and elastic strain ranges, respectively.

According to the Hooke's law [26], the elastic strain range $\Delta \varepsilon_{e}$ can be obtained from dividing the total stress range by the Young's modulus $E$ of the material. Then the plastic strain component $\Delta \varepsilon_{p}$ can be determined by:

$$
\Delta \varepsilon_{p}=\Delta \varepsilon-\frac{\Delta \sigma}{E}
$$




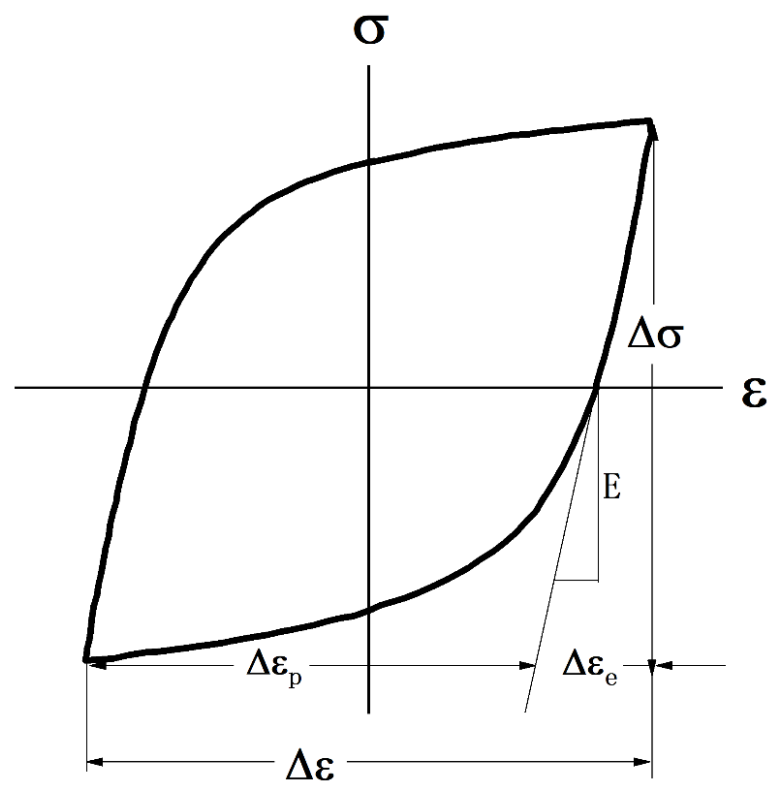

Figure 2. 4 Schematic illustration of the stabilized hysteresis loop for a material.

Particularly, plastic strain can be described by a power law function [27]:

$$
\frac{\Delta \varepsilon_{p}}{2}=\left(\frac{\Delta \sigma}{2 K^{\prime}}\right)^{1 / n^{\prime}}
$$

where $E$ is the young's modulus, $K^{\prime}$ is the hardening coefficient and $n^{\prime}$ is the hardening exponent of cyclically stabilized hysteresis curve. Then the total strain can be rewritten as follows:

$$
\frac{\Delta \varepsilon}{2}=\frac{\Delta \varepsilon_{e}}{2}+\frac{\Delta \varepsilon_{p}}{2}=\frac{\Delta \sigma}{2 E}+\left(\frac{\Delta \sigma}{2 K^{\prime}}\right)^{\frac{1}{n^{\prime}}}
$$

This is known as Ramberg-Osgood equation [27] that considers the cyclic softening and hardening of materials. In addition, experimental fatigue behavior of materials can be characterized by strain-life (e-N) method and stress-life (S-N) method. In LCF region where plastic strain usually has a significant effect on fatigue failure, the relationship 
between plastic strain and fatigue life is described by classic Coffin-Manson equation [7]:

$$
\frac{\Delta \varepsilon_{p}}{2}=\varepsilon_{f}^{\prime}\left(2 N_{f}\right)^{c}
$$

where $\varepsilon_{f}{ }^{\prime}$ is fatigue ductility coefficient and $c$ is fatigue ductility exponent. Conversely, in HCF region where plastic strain has negligible effect on fatigue failure, the relationship between elastic stress and fatigue life can be described by the Basquin equation [7]:

$$
\frac{\Delta \varepsilon_{e}}{2}=\frac{\Delta \sigma}{2 E}=\frac{\sigma_{f}^{\prime}}{E}\left(2 N_{f}\right)^{b}
$$

where $\sigma_{f}{ }^{\prime}$ is the fatigue strength coefficient and $b$ is fatigue strength exponent. Then the total strain amplitude can be expressed by the sum of Basquin equation and Coffin-Manson equation:

$$
\frac{\Delta \varepsilon}{2}=\frac{\sigma_{f}^{\prime}}{E}\left(2 N_{f}\right)^{b}+\varepsilon_{f}^{\prime}\left(2 N_{f}\right)^{c}
$$

The total strain-life equation with the Basquin equation and Coffin-Manson equations is plotted in Figure 2.5: 


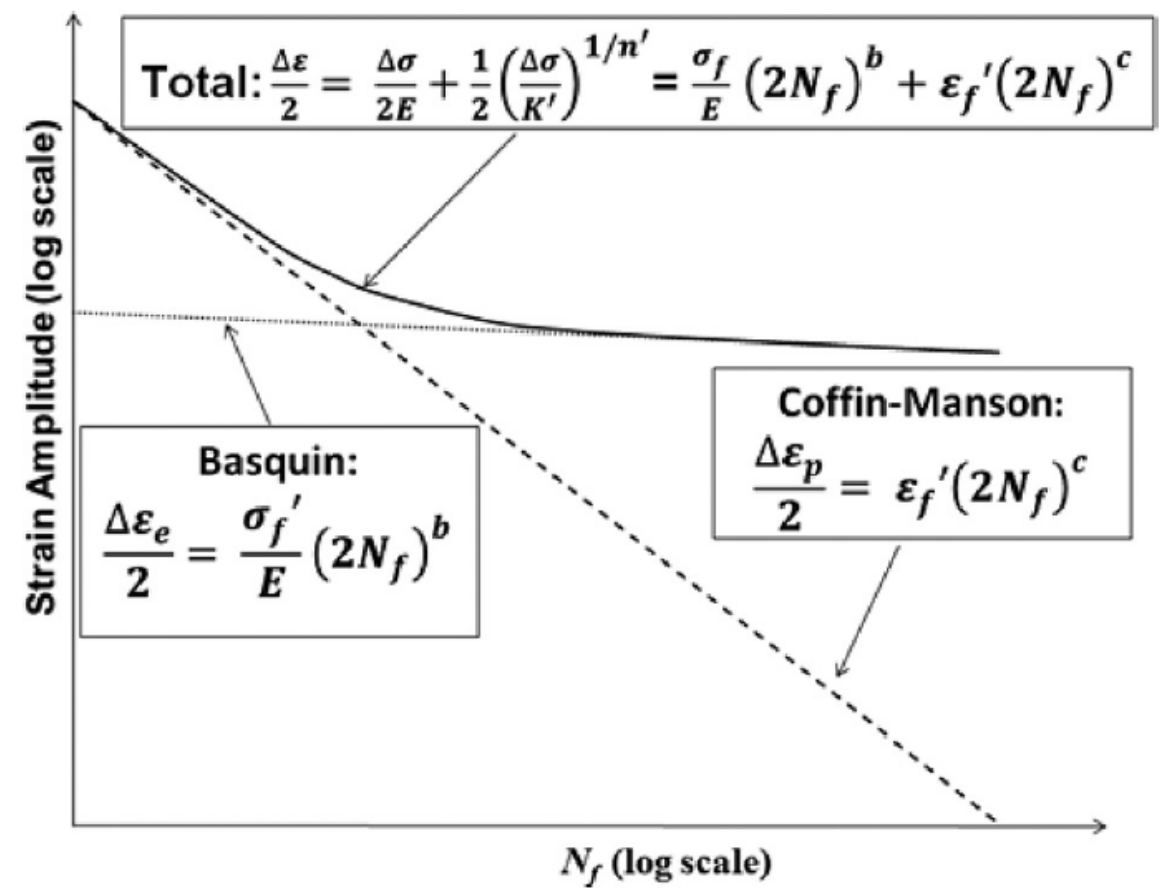

Figure 2. 5 Total strain-life curve composed of Coffin-Manson curve and Basquin curve [28].

The theoretical understanding of material fatigue discussed in this section lays the ground of fatigue crack nucleation analysis in later chapters.

\subsubsection{Fatigue crack nucleation}

As mentioned earlier, fatigue crack nucleation is a priori of fatigue crack initiation. Therefore, primary considerations are given to the crack nucleation mechanism(s) and its origin.

For a defect-free material subjected to cyclic loading, free surfaces are found to provide high incidence of crack nucleation activities. This is caused by two reasons: higher 
concentration of cyclic plastic deformation and reduced constraints near the surface [15]. Consequently, fatigue process is very sensitive to the state of surface, which is affected by the surface finish and environment. In the materials containing defects, lying at or near surface such as pores, second-phase particles and inclusions, however, these defects tend to be the preferable crack nucleation sites due to higher concentration of local plasticity [22] [29]. Major types of surface nucleation sites are persistent slip bands, grain and twin boundaries and inclusions and other inhomogeneities. An example of potential crack nucleation site (PSB) is shown in Figure 2.6.

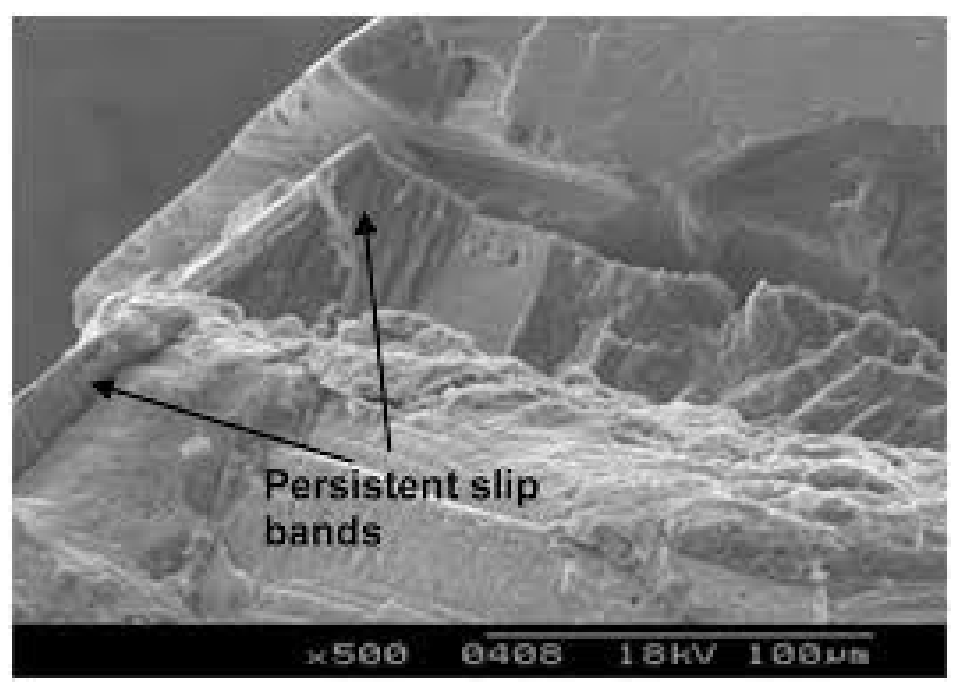

Figure 2. 6 Fatigue crack nucleation from PSBs in aluminum alloy 6063-T6 [111].

Fatigue crack nucleation is associated with cyclic slip localization. This mode varies with materials under different loading conditions. As discussed in previous section, typical cyclic slip irreversibility is associated with PSBs (Figure 2.1 and Figure 2.6). These PSBs 
often result in a noticeable valley and hill topography containing micro-notches, e.g., intrusions and extrusions at the surface [22]. When a material is subjected to cyclic loading, dislocation motion tends to proceed on the favorable slip planes that intersect the free surfaces of the material. A slip step is produced at the material surface when dislocations are emitted, which results in exposure of new fresh surface to the environment, where an oxide layer will form. In addition, as mentioned above, strain hardening may be introduced in the material by cyclic slip, which causes a larger shear stress build-up on the same slip band in the following cycles. Thus, when the loading is reversed, slip reversal is more readily to occur on adjacent slip planes. During this forward slip and slip reversal process, a microcrack may be induced in the form of microscopic intrusion. By the same token, an extrusion can be produced in a similar way. The development of intrusion and extrusion in a material under fatigue condition is schematically shown in Figure 2.7. This mechanism can be repeated in many cycles, which also leads to subsequent crack growth [19][30]. 


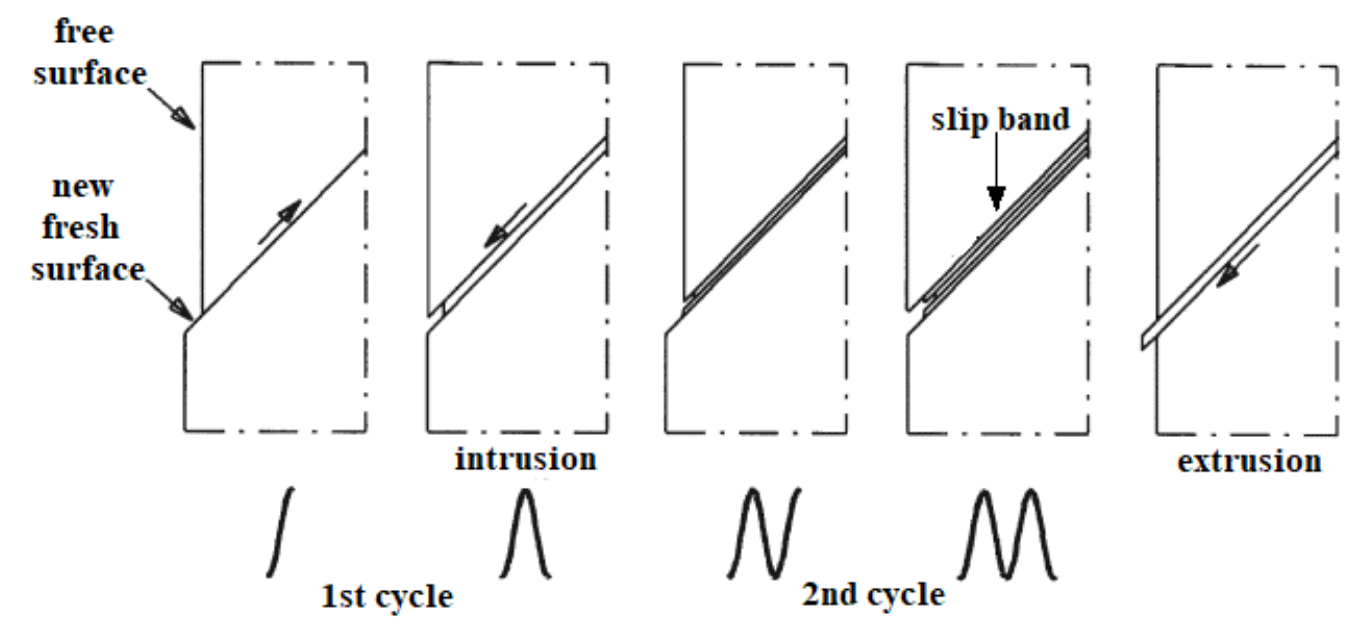

Figure 2. 7 Schematic illustration of cycle slips leading to crack nucleation [19].

Some conceptual models have been proposed to describe the transition from crack nucleation to growth of small cracks. Ma and Laird [31] gave a crack growth model based on the conception of critical degree of strain relaxation. It was created in terms of the fact that during the crack nucleation stage the average spacing between cracks became smaller with increasing plastic strain. The strain would be released when the average spacing reached a critical value, and after the strain relaxation, the strain redistribution would promote the crack growth. However, there was no quantitative description of the crack nucleation process.

While the definition of fatigue crack nucleation is not explicit, the significance of crack nucleation life to total fatigue life cannot be omitted. The crack nucleation and growth of microcracks can predominate the fatigue life of structural materials. According to the study of Makkonen [32], about $40-90 \%$ of total fatigue life of a structural material is spent on 
the crack nucleation process. It is generally believed that crack nucleation during LCF accounts for only a modest portion of the total lifetime, while it can occupy a considerable portion of life in HCF. In LCF, crack nucleation tends to occur on material surface; but in HCF, crack nucleation can occur at subsurface locations, especially around pores or inclusions. As reported by Wang et al. [29] on gigacycle fatigue, crack nucleation was found mostly to occur at subsurface inclusions, which consumed $99 \%$ of the total life for high strength steels.

\subsection{Prediction of Fatigue Crack Nucleation Life}

The methods or models developed to predict the fatigue crack nucleation life of materials and components have been the focus of fatigue research for a long time since Mott identified the origin of fatigue cracks [33].

\subsubsection{Models of fatigue life prediction}

Santecchia et al. reviewed various models of fatigue life prediction that were linked to damage evolution rules or criteria based on stress or strain [34]. Among these models, the Tanaka-Mura model [35] is particularly discussed in detail here, as its further development by $\mathrm{Wu}[9][8]$ lays the theoretical foundation of the present work.

In the Tanaka-Mura model, it is assumed that during cyclic loading, the formation of dislocation pile-ups induced by tension is irreversible on one slip band. The reverse 
dislocation activity with an opposite sign occurs on adjacent slip band during the load reversal. The existing dislocation plie ups on both slip bands may produce back stresses opposing to incoming dislocations. The back stresses are opposite in sign and they facilitate the plastic flow in the subsequent forward and reverse loadings. In this case, a series of vacancy dipoles form and they accumulate with the increasing number of cycles. When the stored strain energy resulting from dislocation accumulation overcomes the surface energy, the dislocation dipoles are transformed into a free surface, which implies the occurrence of fatigue crack nucleation. This process is illustrated in Figure 2.8.

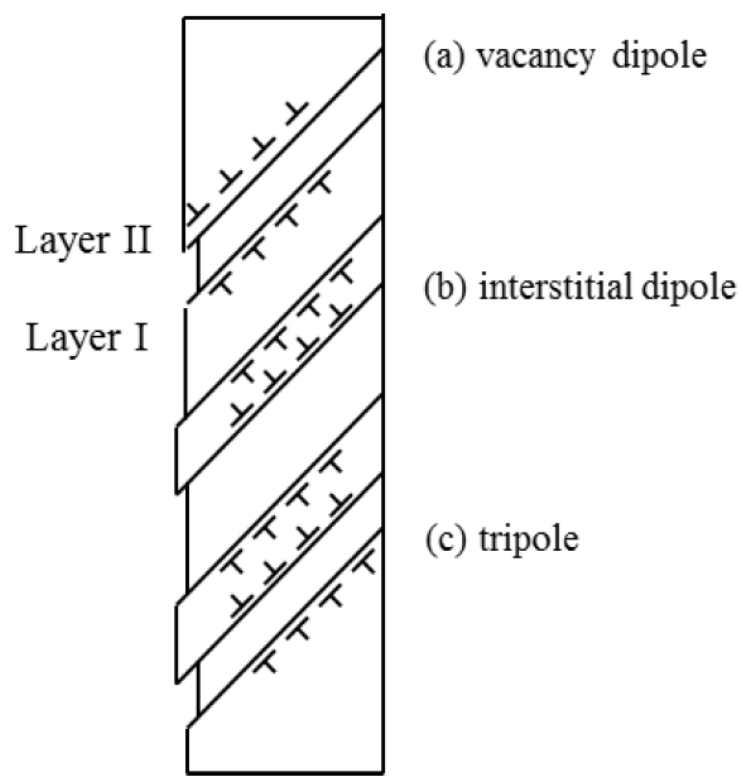

Figure 2. 8 Schematic illustration of dislocation behavior for fatigue crack nucleation: (a) vacancy dipoles (forming an intrusion), (b) interstitial dipoles (forming an extrusion) and (c) tripoles (forming an intrusion-extrusion pair) at the surface [8]. 
In the Tanaka and Mura model, under the first loading maximum shear stress $\tau_{1}$, friction stress $k$ and back stress $\tau_{1}{ }^{D}$ along the slip layer $I$ yield the equilibrium equation:

$$
\tau_{1}^{D}+\tau_{1}-k=0
$$

where $\tau_{1}{ }^{D}$ is the back stress produced by dislocations and it is expressed by:

$$
\left.\tau_{1}^{D}=\frac{\mu b_{v}}{2 \pi(1-v)} \int_{-a}^{a} \frac{D_{1}\left(x^{\prime}\right) d x^{\prime}}{\left(x-x^{\prime}\right)}\right)
$$

where $b_{v}$ is the Burgers vector, $\mu$ is the shear modulus, $v$ is the Poisson's ratio and $a$ is the half grain size. $D_{1}(x)$ is the dislocation distribution within a grain $\left(-a<x^{\prime}<a\right)$. By applying the Muskhelishvili's inversion formula [36] to Eq (2.10), $D_{1}(x)$ can be determined as:

$$
D_{1}(x)=\frac{2(1-v)}{\pi \mu b_{v}} \frac{\left(\tau_{1}-k\right)}{\left(a^{2}-x^{2}\right)^{1 / 2}}
$$

Then the total number of dislocations between $x=0$ and $a$ is:

$$
N_{1}=\int_{0}^{a} D_{1}(x) d x=\left(\tau_{1}-k\right) \frac{2 a(1-v)}{\mu b_{v}}
$$

The plastic displacement $\phi(x)$ caused by dislocation motion is given by the integral:

$$
\phi(x)=\int_{x}^{a} b_{v} D\left(x^{\prime}\right) d x^{\prime}
$$

The total plastic displacement (also known as plastic strain) $\gamma_{1}(-a \leq x \leq a)$ is calculated by Tanaka and Mura, as:

$$
\gamma_{1}=\int_{-a}^{a} \phi(x) d x=\int_{-a}^{a} b_{v} D_{1}(x) x d x=\frac{\pi(1-v)\left(\tau_{1}-k\right) a^{2}}{\mu}
$$

Note that the "plastic strain" $\gamma_{1}$ in Eq (2.15) is obtained by integration of the displacement 
so that it has a physical dimension of $\mathrm{m}^{2}$.

The stored energy associated with the dislocation plie ups on layer $I$ under the first loading can be calculated as:

$$
U_{1}=\frac{1}{2}\left(\tau_{1}-k\right) \gamma_{1}
$$

On reverse loading, the effect of back stress $\tau_{1}{ }^{D}$ on dislocation motion at layer $I I$ needs to be considered. Thus, force balance at layer $I I$ can be expressed as:

$$
\tau_{2}^{D}+\tau_{1}^{D}+\tau_{2}+k=0
$$

where $\tau_{2}{ }^{D}$ is the back stress produced by dislocation plie ups at layer II. Following the similar procedure, plastic strain $\gamma_{2}$ at layer $I I$ can be derived:

$$
\gamma_{2}=-\frac{(1-v)(\Delta \tau-2 k) a^{2} \pi}{\mu}
$$

where $\Delta \tau=\tau_{1}-\tau_{2}$. The stored energy of dislocations at layer $I I$ can be calculated as

$$
U_{2}=-\frac{\gamma_{2}(\Delta \tau-2 k)}{2}
$$

On the $k$-th reversal, the dislocation distribution, $D_{k}(x)$, plastic strain $\gamma_{k}$, and the stored energy $U^{k}$ can be obtained in similar manner:

$$
D_{k}(x)=(-1)^{k+1} \Delta D(x), \gamma_{k}=(-1)^{k+1} \Delta \gamma, U_{k}=\Delta U
$$

where $\Delta D(x)=\frac{2(1-v)(\Delta \tau-2 k) x}{\mu \sqrt{a^{2}-x^{2}}}, \Delta \gamma=\frac{2(1-v)(\Delta \tau-2 k)}{\mu}, \Delta U=\frac{1}{2}(\Delta \tau-2 k) \Delta \gamma$. 
According to the energy criteria, the entire pile-up bursts into a crack once the stored energy in the material volume becomes equal to the energy to form new crack surfaces, as:

$$
2 N_{c} \Delta U=4 a w_{s}
$$

where $N_{c}$ is the fatigue nucleation life and $w_{s}$ is the specific surface energy for unit area. Eventually $N_{c}$ can be derived as

$$
N_{c}=\frac{4 \mu w_{s}}{\pi(1-v) a}(\Delta \tau-2 k)^{-2}
$$

or, in terms of strain:

$$
N_{c}=\frac{4 \pi(1-v) w_{s} a^{3}}{\mu} \Delta \gamma^{-2}
$$

Also note that as $\Delta \gamma$ bears a physical dimension of $\mathrm{m}^{2}, \mathrm{Eq}(2.23)$ is not useable in practice since no strain is measured in unit of $\mathrm{m}^{2}$. If true strain were used in Eq (2.23), the right side of the equation would have additional dimension of $\mathrm{m}^{4}$. Since both Eq (2.22) and Eq (2.23) are based on the invalid expression of plastic strain, the fact sheds doubts on the "righteousness" of these equations. As a matter of fact, the predictability of Eq (2.22) and Eq (2.23) has never been verified in a physically rigorous sense. The stress-based formula, $\mathrm{Eq}(2.22)$, is most often used in engineering analyses for real materials. However, in these analyses, the surface energy $w_{s}$ is renamed as the "specific fracture energy", the value of which is not physically assessed by independent means but relies on fitting to the S-N fatigue curve, e.g., $w_{s}=440 \mathrm{~kJ} / \mathrm{m}^{2}$ for stainless steel [37], and $w_{s}=2 \mathrm{~kJ} / \mathrm{m}^{2}$ for martensitic steel [38][39] [40]. These values are orders-of-magnitude higher than the surface energies 
of metals reported by Tyson and Miller [41]. Additionally, the lattice friction stress $k$ also needs to be determined by fitting to the S-N data obtained from material coupons.

A multiple slip model was developed by Venkataraman et al. [42], which condensed multiple parallel slip bands into one equivalent slip band, and obtained the fatigue crack nucleation life, as:

$$
N_{c}{ }^{\alpha}=0.037\left(\frac{B}{h} \frac{\mu}{\Delta \tau-2 k} \frac{1}{e}\right)\left(\frac{w_{s}}{B \mu}\right)^{0.5}
$$

where $\alpha$ is a composite exponent with value between 0.5 and $1, \Delta \tau$ is the shear stress range, $k$ is the frictional resistance, $\mu$ is the shear modulus, $B$ is the grain size, $e$ is the slip irreversibility factor, $h$ is the slip band width.

Essentially, Eq (2.24) is similar to Eq (2.22), especially when $\alpha=0.5$, but it has several additional parameters such as slip irreversibility factor $e$, slip band width $h$, and exponent $\alpha$. The acquisition of these parameters depends heavily on fitting to the original (experimental) data from literature and use of additional laboratory equipment.

Recently, the Tanaka-Mura model has been improved by $\mathrm{Wu}$ [8] [9], in particular, the dislocation pile-up strain formulation has been revised. By definition, strain is the displacement over the distance where it is measured. In this case, the slip distance is evaluated as the number of dislocations, as expressed by Eq (2.13), times the Burgers vector, i.e., $\Delta l=N_{1} b_{v}$, which occurs over the distance $a^{\prime}$. Thus, the plastic strain due to the dislocation pile-up should be equal to $N_{1} b_{v} / a^{\prime}$, or given by the integral: 


$$
\gamma_{1}=\frac{b_{v}}{a^{\prime}} \int_{0}^{a} D_{1}(x) d x=\frac{2(1-v)\left(\tau_{1}-k\right)}{\mu}
$$

Hence,

$$
\Delta \gamma=\frac{2(1-v)(\Delta \tau-2 k)}{\mu} \text { and } \Delta U=\frac{1}{2}(\Delta \tau-2 k) \Delta \gamma
$$

Again, according to the Griffith energy criterion, once the stored energy in the material volume $\left(b a^{\prime}\right)$ becomes equal to the energy to form new crack surfaces, the entire pile-up bursts into a crack $\left(a^{\prime}\right)$ :

$$
N_{c} \Delta U a^{\prime} b_{v}=2 a^{\prime} w_{S}
$$

from which, the new expression is obtained, as:

$$
N_{c}=\frac{8(1-v) w_{s}}{\mu b_{v}} \frac{1}{\Delta \gamma^{2}}
$$

or

$$
N_{c}=\frac{2 \mu w_{s}}{(1-v)(\Delta \tau-2 k)^{2} b_{v}}
$$

In engineering practice, fatigue life is mostly assessed through uniaxial fatigue testing of material coupons with certain surface roughness. In the uniaxial test, the plastic shear strain has a relationship with the normal plastic strain by the Taylor's factor: $\gamma=\sqrt{3} \varepsilon_{p}$ and $\sigma=\sqrt{3} \tau$ such that the plastic energy is equal for both shear and normal deformation, i.e. $\tau \gamma=\sigma \varepsilon_{p}$. For comparison with the experimental data, a surface roughness factor $R_{S}$ needs to be introduced in the model to take into account the effect of surface finish. Thus, Eq. (2.28) can be converted into 


$$
N_{c}=\frac{8(1-v) R_{S} w_{S}}{3 \mu b_{v}} \frac{1}{\Delta \varepsilon_{p}^{2}}
$$

and by the same token,

$$
N_{c}=\frac{6 \mu R_{S} w_{S}}{(1-v)\left(\Delta \sigma-2 \sigma_{0}\right)^{2} b_{v}}
$$

where $\sigma_{0}=\sqrt{3} k$. Eq (2.30) has been validated against low cycle fatigue (LCF) data of type 316 stainless steel with different surface finishing, as shown in Figure 2.9. The theoretical prediction of Eq (2.30) exactly matches the experimental data for the electropolished surface obtained by Wareing and Vaughan [43], which represents an "ideal" surface finish. By comparison, the machined surface roughness has an effect of $R_{S} \sim 1 / 3$. Also, Eq (2.30) life prediction has also been compared with uniaxial LCF lives for several metals and alloys at room temperature, as illustrated in Figure 2.10. For these materials, the roomtemperature surface energies are estimated based on the data provided by Tyson and Miller [41] for the base metal at the homologous temperature (temperature of a material as a fraction of its melting point temperature using the Kelvin scale) $\sim 0.25$. The expression for surface energy is given by [41]:

$$
w_{s}=w_{s}\left(T_{m}\right)+\phi(T) \frac{R T_{m}}{A}
$$

where $w_{s}\left(T_{m}\right)$ is the surface energy at the melting temperature, $A$ is the surface area per mole of surface atom, $\phi(T)$ is a temperature-dependent parameter and $R$ is the Boltzmann constant.

The surface roughness factor $R_{S}=1 / 3$ (assuming the same machined condition). The 
material property parameters and the calculated values of the fatigue life coefficient for some metals are reported in Table 2.1; all materials are assumed to have a Poisson's ratio of 0.3 . The theoretical predictions reportedin Table 2.1 are in very good agreement with the experimental data for Type 316 stainless steel [43], copper [44], titanium [45], tungsten [46], Waspaloy [47], MAR-M 509 [48] [49]. As shown in Figure 2.10, the fatigue life lines of different metals are not far apart. For Waspaloy, the data from coarse grained (CG) microstructure with the grain size of $125 \mu \mathrm{m}$ and fine grained (FG) microstructure with the grain size of $16 \mu \mathrm{m}$ do not show much difference either in the LCF life vs. plastic strain plot, whereas the original Tanaka-Mura model, Eq (2.23), would predict a grain size dependence to the power of 3 . Therefore, Eq (2.30) can predict the LCF life of a structural material only based on the known material properties of modulus, surface energy and Burgers vector without resorting to fatigue testing.

The stress-based formula, $\mathrm{Eq}$ (2.31), contains one microstructure-dependent constant, $\sigma_{0}=$ $\sqrt{3} k$, which is related to the critical resolved shear stress, $k$, by a Taylor's factor and it generally follows the Hall-Petch dependence on the grain size. In the present study, the effects of microstructure in terms of grain orientation on the fatigue life of materials will be further studied using finite element modelling approach. 


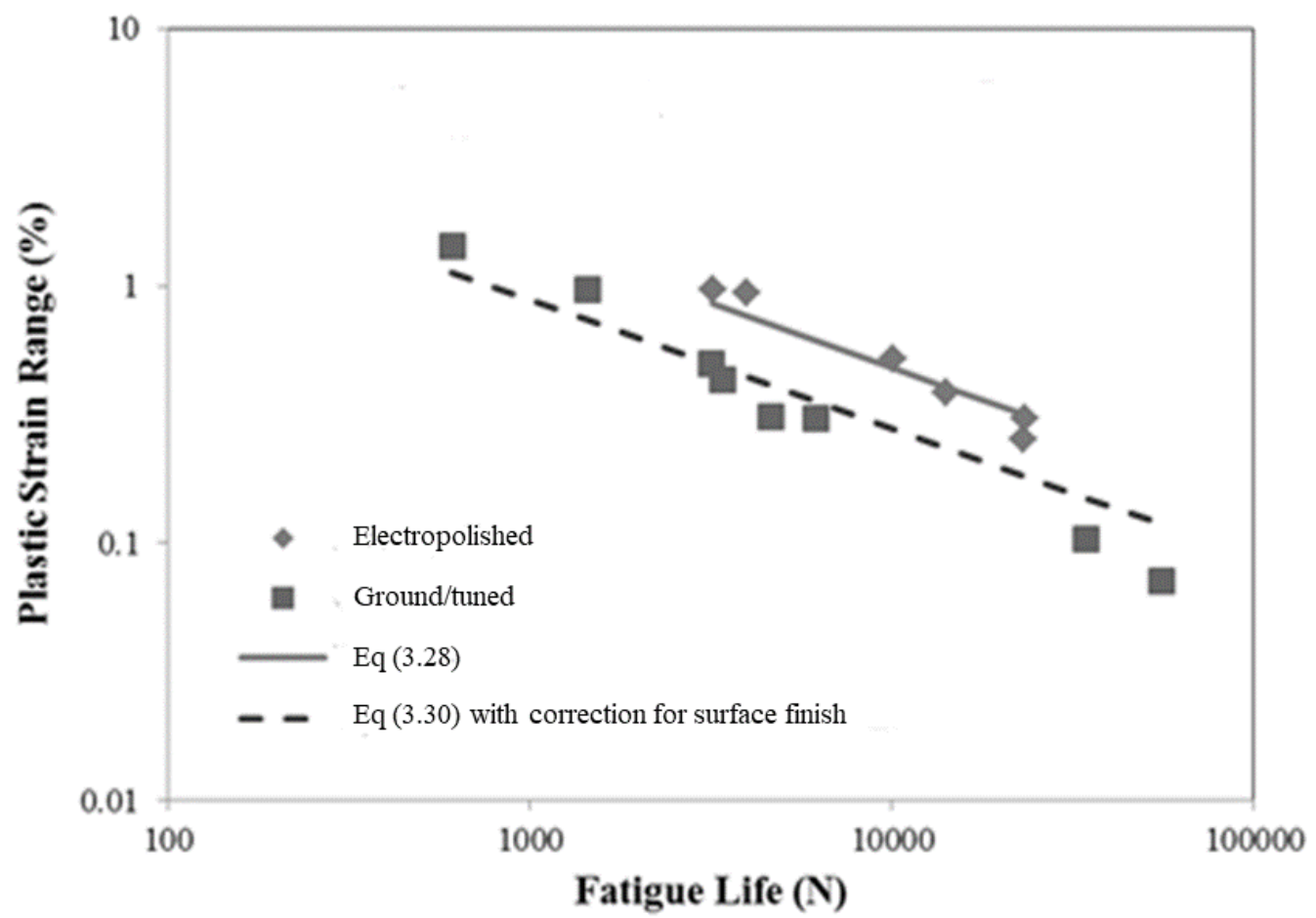

Figure 2.9 Fatigue life of Type 316 stainless steel with different surface finishing. (The symbols represent the referenced experimental data and the lines represent theoretical predictions of Eq (2.28) and $\mathrm{Eq}(2.30))$ [8].

Table 2. 1 Fatigue coefficients of metals/alloys [8]

\begin{tabular}{|c|c|c|c|}
\hline Material & $\begin{array}{c}\text { Young's } \\
\text { modulus E } \\
(\mathrm{GPa})\end{array}$ & $\begin{array}{c}\text { Burger's vector } \\
\mathrm{b}_{\mathrm{v}} \\
\left(10^{-10} \mathrm{~m}\right)\end{array}$ & $\frac{8(1-v) \mathrm{R}_{\mathrm{s}} \mathrm{w}_{\mathrm{s}}}{3 \mu \mathrm{b}_{\mathrm{v}}}$ \\
\hline $\mathrm{Cu}$ & 112 & 2.56 & 0.099 \\
\hline $\mathrm{Ti}$ & 54.5 & 3.21 & 0.181 \\
\hline $\mathrm{W}$ & 286 & 2.74 & 0.066 \\
\hline $\mathrm{Fe}$ (Type 316 stainless steel) & 199 & 2.48 & 0.117 \\
\hline $\mathrm{Ni}$ (Waspaloy) & 211 & 2.48 & 0.072 \\
\hline $\mathrm{Co}$ (Mar-M 509) & 211 & 2.48 & 0.077 \\
\hline
\end{tabular}




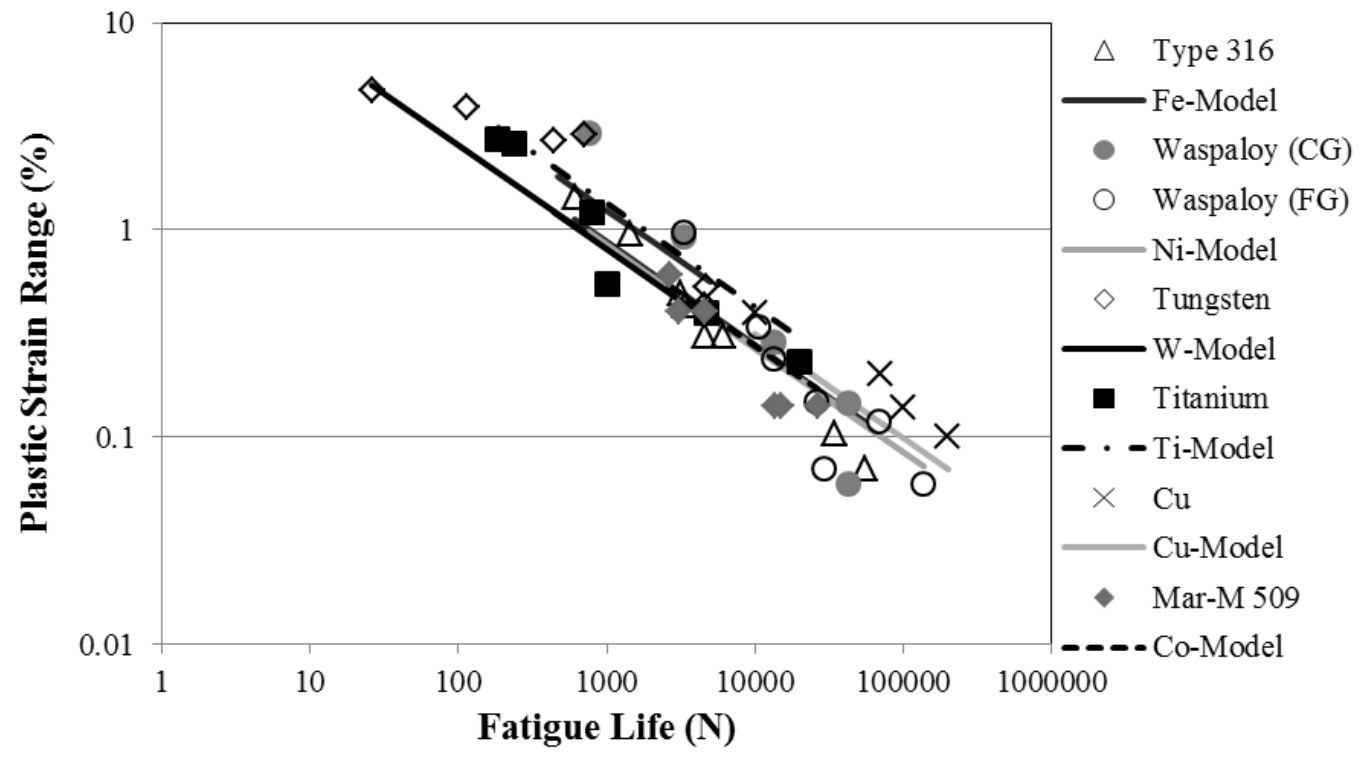

Figure 2. 10 Predicted fatigue curves in comparison with experimental data for various materials [8].

\subsubsection{Fatigue life prediction with FEM or FEA}

Traditionally, fatigue lives of engineering materials are assessed via fatigue testing, either in stress or strain-control mode. Several ASTM standards [6][50] have been developed to standardize the testing procedure thus to ensure the correctness of the data generation. Usually, fatigue test data have a large scatter, typically by a factor of 2 , due to the variabilities in material microstructure, properties, and surface finish of the coupon. Therefore, a large number of coupons are often required in fatigue testing to obtain reliable data, which certainly costs a great deal of time and money. Particularly for fatigue durability design of aircraft, hundreds of materials need to be fatigue-tested but only to generate the basic design data, even before the aircraft takes shape in drawing. Moreover, experimental investigation may not be possible for some service conditions owing to the 
limitations of laboratory equipment and environment which may oversimplify the real working condition.

For the full airplane, certification tests for aircraft structures are necessary to ensure the load carrying capability and performance of each component. However, hardware testing is costly and delays the design cycle. In the current certification procedures, a limited number of full-scale tests are performed, and the stochastic nature of structural failures is not captured. The full process of aircraft certification from material characterization to full scale testing often takes approximately 20 years with a huge cost, which becomes unaffordable to the Original Equipment Manufacturer (OEM). Therefore, the federal aviation regulations are preparing to allow analysis as a means of compliance to solve the structural problems and provide acceptable means of validation of the analysis. This process is called "certification-by-analysis" [51].

With the development of robust computational technologies and ample computer resources, finite element method (FEM) or finite element analysis (FEA) provides an efficient approach to numerical simulation and analysis. FEM has found successful applications in structural analysis and FEM software has become an indispensable tool in computer-aided engineering design. Nowadays, increasing FEM analyses are being performed to evaluate the material performance and durability. However, the reliability of these solutions is always a major concern. The main issues lie in whether constitutive modeling and life prediction can faithfully reflect the physics of material deformation and failure or not. 
Nevertheless, FEM analyses provide tremendous time and cost savings in design, and with improvement in modeling techniques, can lead to satisfactory results with controlled deviations.

Analysis of fatigue crack nucleation by FEM has received growing interest in recent years. Ample research has been conducted and the results on various materials have been achieved. Briffod et al. [52] performed fatigue crack nucleation life prediction for pure iron polycrystalline aggregate. The grain structure was generated by two dimensional (2D) Voronoi tessellation. The stress distribution under cyclic loading was obtained from simulation. The fatigue crack nucleation life was calculated using the Tanaka-Mura model. Bruckner-Foit and Huang [39] proposed a method to estimate material parameters based on the Tanaka-Mura crack initiation law with FEM. The material used in their study was a martensitic steel with lath martensite in the austenite matrix. The prior austenite grains were represented by a random cell structure using Voronoi tessellation. The potential crack nucleation paths were simulated along the crystallographic slip planes of martensite phase. The resolved shear stress was evaluated by averaging the shear stresses over the length of a specific slip band, which acted as the potential crack nucleation site. Fatigue crack nucleation life was then calculated by substituting the value of the resolved shear stress and the length of critical slip band into the Tanaka-Mura equation [53]. Giang [54] carried out an investigation in fatigue crack incubation (nucleation) at carbides of forged M3:2 tool steel. The analysis was performed using a Coffin-Manson type criterion which involved 
cyclic plastic shear strain range and material dependent parameters. A 2D representative volume element (RVE) was employed for the material which contained two phases (carbides and martensite) having different material properties. The cyclic plastic shear strain was evaluated by averaging the values over an area of local plastic zone at the tip of carbides. The material-dependent coefficients were estimated by applying a regression fitting of the LCF experimental data obtained from the Brondsted's work [55]. Finally, the fatigue crack nucleation life was computed by substituting these parameters into the CoffinManson type equation. In another study by Seiichiro [56], the fatigue crack nucleation life of aluminum alloy joints was characterized by a criterion demonstrating an exponential relationship between the total strain range and crack nucleation life based on the previous experimental data [57]. In order to simulate fatigue testing on the weld join, a 2D geometry with three different angular distortions was created. The local strain ranges from the numerical analysis were used in the exponential expression to calculate the crack nucleation life. The simulation results were compared with the experimental results in the same stress ranges.

All the aforementioned analyses revealed the local stress and strain effects on fatigue crack nucleation at microstructural level, but the life prediction parameters still rely on the fatigue test data, so the "prediction" is not class-A. This thesis study is carried out with a focus on pure computational prediction of microstructural fatigue crack nucleation with respect to the effect of microstructure (grain orientation) on the fatigue crack nucleation life. 


\subsection{Microstructural Modeling}

The microstructural modeling for fatigue analysis has two aspects: i) the constitutive law(s) for material behavior at microstructural level, since grains are individual crystallites that exhibit anisotropic properties; and ii) an automated microstructure construction method such as Voronoi tessellation technique combined with random or selected orientation assignment within a material RVE.

\subsubsection{Theory of anisotropic elasticity}

The materials with crystallographic direction dependence are generally anisotropic materials [58]. Their elastic and plastic behavior is described by classical theories of anisotropic elasticity and plasticity.

For three dimensional (3D) elastic bodies, the anisotropic stress-strain relations can be represented by the generalized Hooke's law [59]:

$$
\sigma_{i j}=C_{m n} \varepsilon_{k l}
$$

where $\sigma_{i j}$ and $\varepsilon_{k l}$ are notations of stress and strain respectively and $C_{m n}$ is material stiffness. The generalized Hooke's law can also be expressed in the matrix form, as: 


$$
\left[\begin{array}{l}
\sigma_{11} \\
\sigma_{22} \\
\sigma_{33} \\
\sigma_{23} \\
\sigma_{12} \\
\sigma_{13}
\end{array}\right]=\left[\begin{array}{llllll}
C_{11} & C_{12} & C_{13} & C_{14} & C_{15} & C_{16} \\
C_{21} & C_{22} & C_{23} & C_{24} & C_{25} & C_{26} \\
C_{31} & C_{32} & C_{33} & C_{34} & C_{35} & C_{36} \\
C_{41} & C_{42} & C_{43} & C_{44} & C_{45} & C_{46} \\
C_{51} & C_{52} & C_{53} & C_{54} & C_{55} & C_{56} \\
C_{61} & C_{62} & C_{63} & C_{64} & C_{65} & C_{66}
\end{array}\right] *\left[\begin{array}{c}
\varepsilon_{11} \\
\varepsilon_{22} \\
\varepsilon_{33} \\
\varepsilon_{23} \\
\varepsilon_{12} \\
\varepsilon_{13}
\end{array}\right]
$$

where $\sigma_{i j}$ and $\varepsilon_{k l}$ with two subscripts are the notations of stress and strain respectively and $36 C_{m n}$ terms are elastic stiffness tensor elements. The stiffness tensor can be either constant for a homogeneous body or variable for non-homogenous body. It can be seen from $\mathrm{Eq}(2.34)$ that each stress component is linearly dependent on all strain components. Due to material crystal symmetries, Eq (2.34) can be simplified as follows:

$$
\left[\begin{array}{l}
\sigma_{11} \\
\sigma_{22} \\
\sigma_{33} \\
\sigma_{23} \\
\sigma_{12} \\
\sigma_{13}
\end{array}\right]=\left[\begin{array}{cccccc}
C_{11} & C_{12} & C_{13} & C_{14} & C_{15} & C_{16} \\
& C_{22} & C_{23} & C_{24} & C_{25} & C_{26} \\
& & C_{33} & C_{34} & C_{35} & C_{36} \\
& s y m & & C_{44} & C_{45} & C_{46} \\
& & & & C_{55} & C_{56} \\
& & & & & C_{66}
\end{array}\right] *\left[\begin{array}{c}
\varepsilon_{11} \\
\varepsilon_{22} \\
\varepsilon_{33} \\
\varepsilon_{23} \\
\varepsilon_{12} \\
\varepsilon_{13}
\end{array}\right]
$$

Although the elastic constants have been reduced from 36 to 21 , it is still very challenging to model fully anisotropic materials. Fortunately, many materials are not entirely anisotropic so that material crystal symmetries allow further simplification of the equation.

For orthotropic materials, the expression of stress and strain relations is given as:

$$
\left[\begin{array}{l}
\sigma_{11} \\
\sigma_{22} \\
\sigma_{33} \\
\sigma_{23} \\
\sigma_{12} \\
\sigma_{13}
\end{array}\right]=\left[\begin{array}{cccccc}
C_{11} & C_{12} & C_{13} & 0 & 0 & 0 \\
C_{12} & C_{22} & C_{23} & 0 & 0 & 0 \\
C_{13} & C_{23} & C_{33} & 0 & 0 & 0 \\
0 & 0 & 0 & C_{44} & 0 & 0 \\
0 & 0 & 0 & 0 & C_{55} & 0 \\
0 & 0 & 0 & 0 & 0 & C_{66}
\end{array}\right] *\left[\begin{array}{c}
\varepsilon_{11} \\
\varepsilon_{22} \\
\varepsilon_{33} \\
\varepsilon_{23} \\
\varepsilon_{12} \\
\varepsilon_{13}
\end{array}\right]
$$

For orthotropic materials with cubic symmetry, the stiffness tensor constants can further 
reduce to:

$$
\begin{aligned}
& C_{11}=C_{22}=C_{33}, \\
& C_{44}=C_{55}=C_{66}, \\
& C_{12}=C_{13}=C_{23} .
\end{aligned}
$$

Ultimately, the number of the independent constants in the matrix is minimized to only three.

\subsubsection{Yielding criteria and hardening law}

The generalized Hooke's law well defines the relationship between stress and strain when materials are elastically deformed. However, plastic deformation will occur once the stress exceeds the limit of elasticity of the materials. The yielding criteria are used to define the onset of plastic deformation and describe the ductile behavior of materials in the plastic regime. For isotropic materials, a yielding criterion, named Von Mises failure theory, finds its applications for most materials, which is expressed as [60]:

$$
\left(\sigma_{1}-\sigma_{2}\right)^{2}+\left(\sigma_{2}-\sigma_{3}\right)^{2}+\left(\sigma_{1}-\sigma_{3}\right)^{2}=2{\sigma_{s}}^{2}
$$

where $\sigma_{1}, \sigma_{2}$ and $\sigma_{3}$ are the three principal stresses, and $\sigma_{s}$ is the yielding strength of the material.

For anisotropic materials, Von Mises criterion fails because the yielding behavior varies in different directions [61]. Neglecting the influence of superposition of hydrostatic pressure, Hill [62] introduced the plastic potential criterion for anisotropic materials in the following 
form:

$$
2 f \equiv F\left(\sigma_{22}-\sigma_{33}\right)^{2}+G\left(\sigma_{33}-\sigma_{11}\right)^{2}+H\left(\sigma_{11}-\sigma_{22}\right)^{2}+2 L{\sigma_{23}}^{2}+2 M{\sigma_{31}}^{2}+2 N{\sigma_{12}}^{2}=1(
$$

where $f$ is regarded as the yield function in the Cartesian system, while $F, G, H, L, M, N$ are six constants characterizing the material's anisotropic yield strength in the respective direction, which are usually determined experimentally. For a material of cubic symmetry, the following relations exist:

$$
\begin{aligned}
& F=G=H \\
& L=M=N
\end{aligned}
$$

An example of the calculation of the anisotropic material parameters according to the Abaqus manual will be demonstrated in detail in later chapter to define the yielding behavior of polycrystalline nickel-based superalloy, Haynes 282 .

Usually, as plastic deformation continues, the material becomes work-hardened. A hardening law is needed to update the yield condition with the progression of plastic deformation. Generally, there are two basic types of hardening: isotropic hardening and kinematic hardening, which describe the evolution of yield surface, as illustrated in Figure 2.11. In isotropic hardening, the yield surface dilates without changing its shape and the expansion of the yield surface is isotropic in all loading directions and the origin of the yield surface remains stationary. In a mathematical sense, the yield function for isotropic hardening takes the form [63]: 


$$
\mathrm{f}\left(\sigma_{i j}, K_{i}\right)=f_{0}\left(\sigma_{i j}\right)-K\left(\varepsilon_{i j}\right)=0
$$

where $f_{0}\left(\sigma_{i j}\right)$ is the yield function and $K\left(\varepsilon_{i j}\right)$ is the hardening parameter describing the size of the yield surface changing with the strain.

In kinematic hardening, the yield surface translates in the direction of the loading path without changing its shape or size. The yield function in this case takes the form:

$$
\mathrm{f}\left(\sigma_{i j}, K_{i}\right)=f_{0}\left(\sigma_{i j}-\alpha_{i j}\left(\varepsilon_{i j}\right)\right)-K_{0}=0
$$

where the origin of the initial yield surface is shifted by $\alpha_{i j}$, which is named back stress, depending on the strain, and $K_{0}$ denotes the size of the initial yield surface.

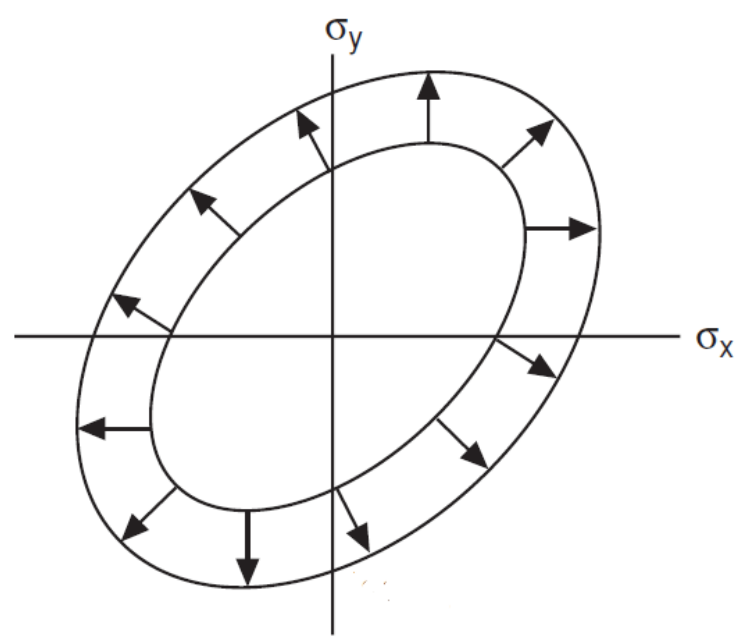

(a)

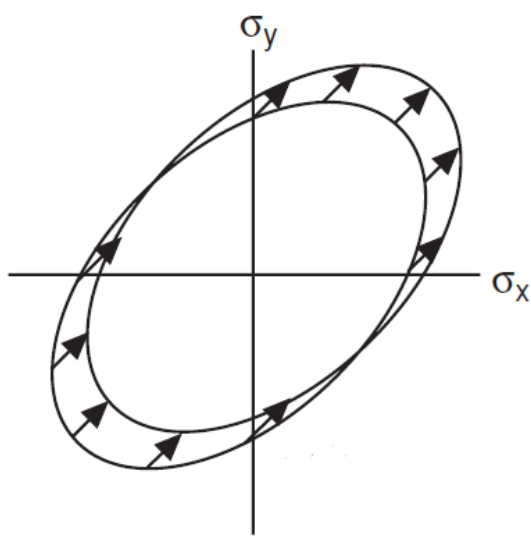

(b)

Figure 2. 11 Schematic illustration of (a) isotropic hardening law and (b) kinematic hardening law. 


\subsubsection{Crystal plasticity theory}

The crystal plasticity theory has been developed for modeling deformation at microscale of materials, which treats the total deformation as a resultant of lattice stretching and rotation, and plastic shearing due to crystallographic slip.

\subsubsection{Definitions of crystallography}

A crystal structure is the structure of atoms, ions or molecules that are arranged in an ordered manner [26]. The most common crystal structures of metals are face-centered cubic (FCC), body-centered cubic (BCC) and hexagonal closest packed (HCP), as illustrated in Figure 2.12 .

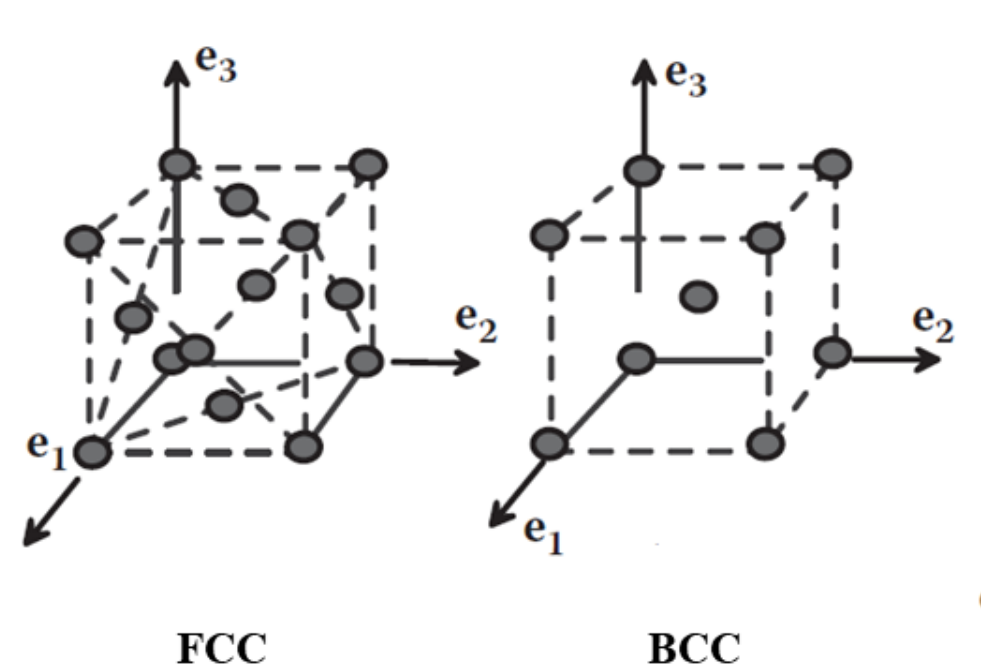

(a)

(b)

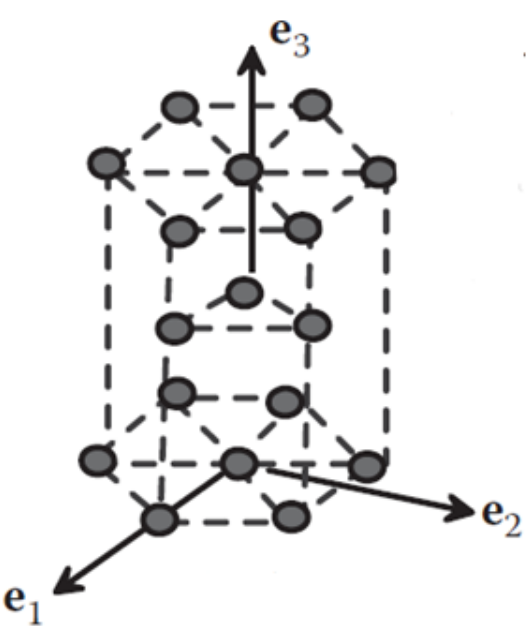

HCP

(c)

Figure 2.12 Crystal structures for common metals: (a) FCC structure, (b) BCC structure and (c) HCP structure [64]. 
For a cubic lattice material, the position of all atoms in the crystal structure can be defined by $h e_{1}+k e_{2}+l e_{3}$, where $e_{1}, e_{2}$ and $e_{3}$ are the three base vectors of the unit cell. The lengths of these vectors are called lattice constants, $h, k$ and $l$, which are integer or fractional numbers, depending on the location of the atom. The planes and directions in a single crystal lattice can be expressed in a similar manner by the Miller index notations. In terms of the notations, a direction parallel to a vector $\left(h e_{1}+k e_{2}+l e_{3}\right)$ can be denoted by its components $[h k l]$, where $h, k$ and $l$ are positive integers. Its reverse direction is represented by $[\bar{h} \bar{k} l]$. The family of such defined directions is represented by $\langle h k l\rangle$. A plane with its normal in the direction of $\left(h e_{1}+k e_{2}+l e_{3}\right)$ can be represented by $(h k l)$ and its family by $\{h k l\}$.

In order to characterize and quantify $3 \mathrm{D}$ crystallographic directions by a geometrical method, stereographic projection can be employed to plot these directions on a 2D plane. There are two applications of stereographic projection in crystallography: 1) pole figure and 2) inverse pole figure. In the former, poles are usually stereographic projections of crystallographic directions of interest with respect to the sample reference axes including rolling direction, normal direction and transverse direction. Figure 2.13 is provided to visualize how a pole figure is plotted. 


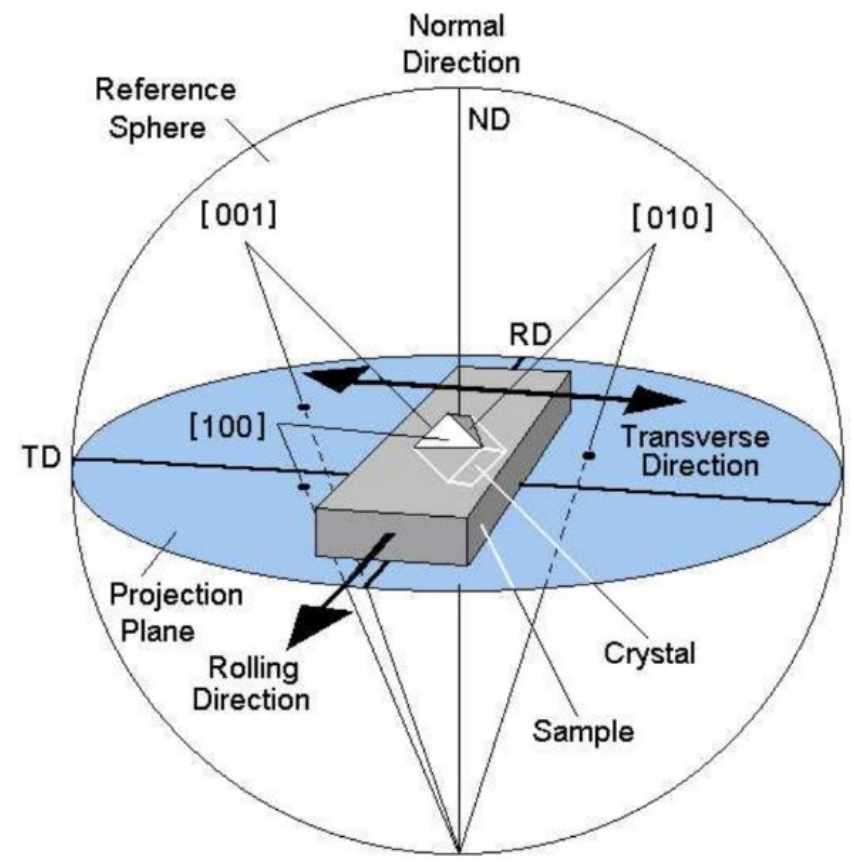

Figure 2. 13 Principle of pole figure plotting [65].

It can be seen that a grain in a sample is placed in the center of the reference sphere with unit radius and the sample axes are aligned with the orthogonal coordinate axes of the reference sphere. When the directions of interest intersect with the reference sphere at certain points, the lines starting from these points are then projected to the bottom of the reference sphere. The intersections between the lines and projection planes are where poles are plotted.

The latter is constructed similarly to the pole figure, but the major difference is that in an inverse pole figure the directions of interests are plotted with respect to the crystal axes rather than the sample axes. Figure 2.14 shows an example of inverse pole figure for cubic crystal which is divided into 24 curvilinear triangles. Each of these triangles has $\langle 100\rangle$, 
$\langle 110\rangle$ and $\langle 111\rangle$ directions at its corners and they are equivalent to each other due to the symmetry of the crystal. Therefore, usually only a standard tringle is displayed to represent an inverse pole figure.

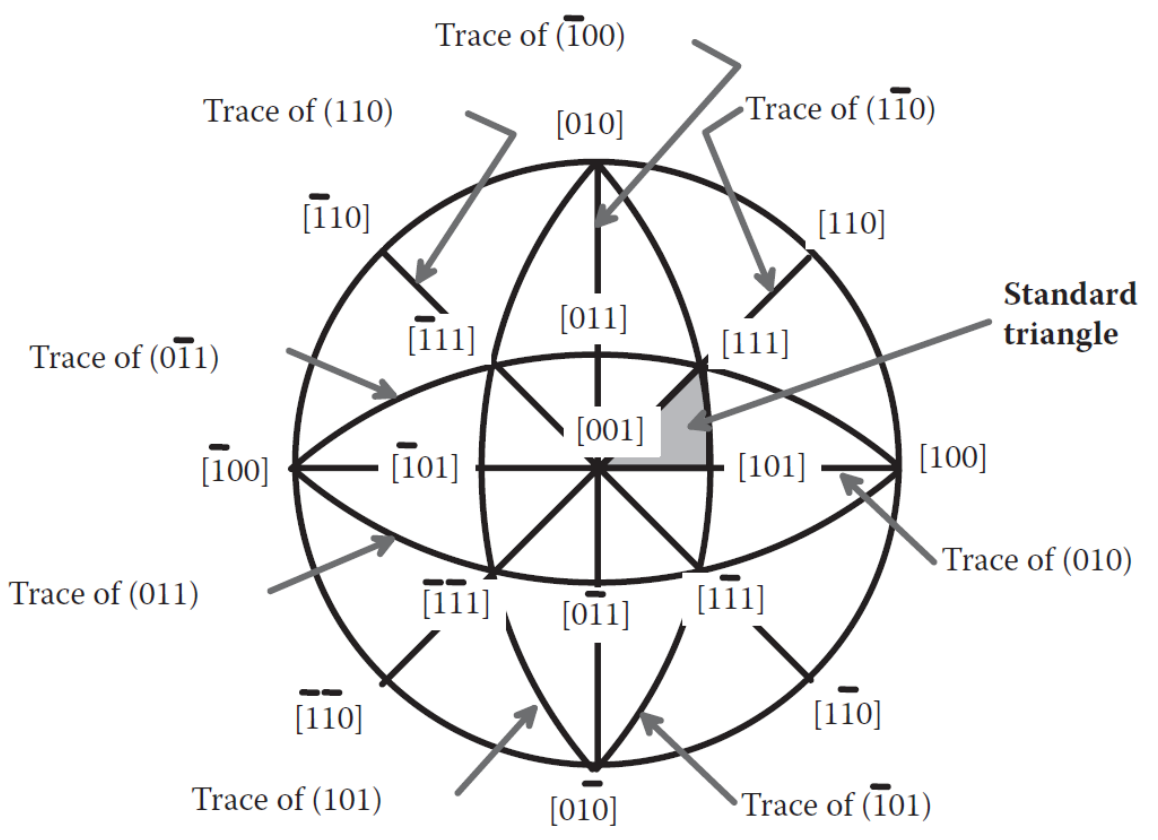

Figure 2.14 An example of inverse pole figure [64].

\subsubsection{Single crystal plasticity}

The displacements in a crystal can always be described by the deformation gradient $F$ with components of $F_{i j}=\partial x_{i} / \partial X_{j}$, where $X_{j}$ and $x_{i}$ denote the reference and current particle positions, respectively. $F$ can be decomposed into elastic and plastic parts [64]:

$$
F=F^{e} F^{p}
$$

where the plastic part $F^{p}$ is assumed to result in lattice shearing due to slip while the elastic 
part $F^{e}$ is accountable for lattice stretching and rotation. This decomposition is schematically shown in Figure 2.15.

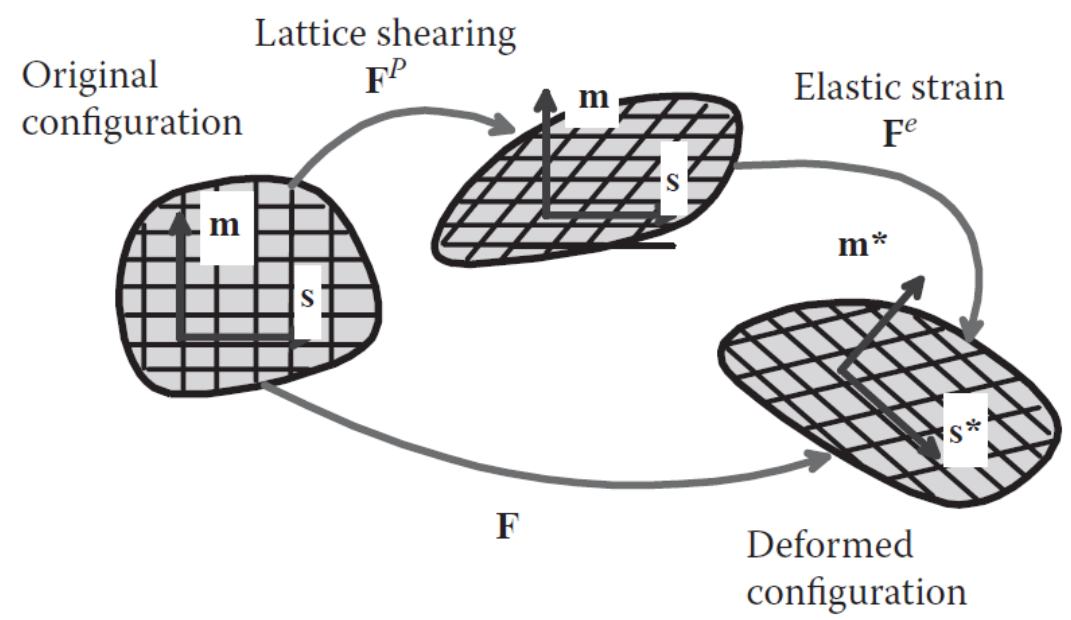

Figure 2. 15 Decomposition of crystal deformation into elastic and plastic parts [64].

Lattice shearing tends to occur in one of family members of crystallographic directions which are known as the slip directions. The planes on which these slip directions lie are called slip planes. All symmetrically identical slip planes and associated family of slip directions are so-called slip systems. For describing crystallographic slip, slip systems are usually defined in terms of Miller indices. For fcc crystals, the most densely packed planes are the diagonal planes of the unit cell, as indicated in Figure 2.12(a). The full family of slip systems in an fcc crystal may be written as $\langle 1 \overline{1} 0\rangle\{111\}$. There are 12 such systems in an fcc crystal (four planes and each with three directions).

The velocity $L$ is determined by the rate of change for $F$ as follows: 


$$
L=\dot{F} \cdot F^{-1}
$$

The rate of change for $F^{p}$ can be expressed in terms of the rate of shear in the $\alpha-$ th activated slip system $\dot{\gamma}^{\alpha}$ :

$$
L^{p}=\sum_{\alpha=1}^{N} \dot{\gamma}^{\alpha} \cdot s^{\alpha} \otimes m^{\alpha}
$$

where the sum represents the total number of slip systems and vectors $s^{\alpha}$ and $m^{\alpha}$ denote the slip directions and the normal to the slip plane, respectively. The resolved shear stress can be calculated from the Cauchy stress tensor:

$$
\tau^{\alpha}=\sigma:\left(s^{\alpha} \otimes m^{\alpha}\right)_{s y m}
$$

The velocity gradient in the deformed state of the crystal can be decomposed into a symmetric stretching part $D$ and an antisymmetric spin part $W$ :

$$
L=D+W
$$

The stretching tensor $D$ and spin tensor $W$ can also be decomposed into elastic and plastic parts:

$$
D=D^{e}+D^{p} ; W=W^{e}+W^{p}
$$

Then the following set of equations can be obtained:

$$
\left\{\begin{array}{c}
D^{e}+W^{e}=\dot{F}^{e} \cdot F^{e-1} \\
D^{p}+W^{p}=\sum_{\alpha=1}^{N} \dot{\gamma}^{\alpha} \cdot s^{\alpha} \otimes m^{\alpha}
\end{array}\right\}
$$

Finally, a set of plastic constitutive equations can be introduced to describe the relationship between slip rate $\dot{\gamma}^{\alpha}$ of each slip system and stress in the crystal. The application of these 
equations highly depends on the loading condition applied. For cyclic loading, the slip rate $\dot{\gamma}^{\alpha}$ can be expressed as follows:

$$
\dot{\gamma}^{\alpha}=\dot{\gamma}_{0}{ }^{\alpha} \cdot\left|\frac{\tau^{\alpha}-\chi^{\alpha}}{g^{\alpha}}\right|^{n} \operatorname{sign}\left(\tau^{\alpha}-\chi^{\alpha}\right)
$$

where $n$ is the power-law exponent, $g^{\alpha}$ and $\chi^{\alpha}$ denote the isotropic and kinematic hardening, respectively, which describe the evolution of strain hardening. $g^{\alpha}$ and $\chi^{\alpha}$ are given by:

$$
\begin{gathered}
\dot{g}^{\alpha}=\sum_{1}^{\beta} h_{\alpha \beta} \cdot \dot{\gamma}^{\beta} \\
\dot{\chi}^{\alpha}=p \dot{\gamma}^{\alpha}-r \chi^{\alpha}\left|\dot{\gamma}^{\alpha}\right|
\end{gathered}
$$

where self and latent hardening rates are given by:

$$
\begin{gathered}
h_{\alpha \alpha}=h\left(\gamma_{a}\right)=h_{0} \cdot \sec ^{2}\left|\frac{h_{0}}{\tau_{s}-\tau_{0}}\right|, \alpha=\beta \\
h_{\alpha \beta}=q \cdot h\left(\gamma_{a}\right), \alpha \neq \beta
\end{gathered}
$$

where $r, p$ and $q$ are material constants, $h_{0}$ is the initial hardening modulus, $\tau_{s}$ is the breakthrough stress and $\tau_{0}$ is the yield stress. $\gamma_{a}$ denotes the accumulated shear strain in all slip systems and is expressed as:

$$
\gamma_{a}=\int_{0}^{t} \sum_{\alpha=1}^{N}\left|\dot{\gamma}^{\alpha}\right| d t
$$

The fundamental flow rule aforementioned does provide a physics-based understanding of single crystal plasticity resulting from multiple slips accommodated by active slip systems. However, it should be noted that this rule is rate-dependent. When rate-dependent behavior 
is dominant, a large value of exponent $n$ is usually required, which can reduce computational efficiency of the model [66]. In addition, the power law flow rule and the associated evolutionary equation for back stresses, Eq (2.51) to Eq (2.55), employ a hoard of material parameters, which are difficult to collect. Lacking material data in this regard can be a hurdle to apply this rule on new materials. In comparison, Hill's yield theory suggests an easier way for parameter determination. Considering the symmetrical FCC crystal structure of nickel-based single crystal investigated in the present study, only uniaxial yield stresses from tensile tests in two different directions are required for parameter determination. Moreover, implementation of Hill's yield theory as a classic yield criterion describing anisotropic plastic deformations is rather user-friendly with finite element codes, because it is built in commercial FEM software, e.g. Abaqus, which is chosen as the analysis tool in the present study. Using Abaqus package to implement the Hill's yield theory in modelling of anisotropic plasticity at the grain level displays simplicity and convenience with adequate accuracy.

\subsubsection{Representation of polycrystalline structure}

Construction of polycrystalline structure by computational techniques is the prerequisite to fatigue crack nucleation modeling of polycrystalline materials by FEM. Therefore, the basic concepts of a Representative Volume Element (RVE) and Voronoi tessellation technique are reviewed below. 


\subsubsection{Representative volume element (RVE)}

The representative volume element (RVE) of a material is a material volume that contains a set of microscopic constituents and microstructures, which involve grains, inclusions, microcracks and voids and other defects [67]. RVE is often used to represent heterogeneous microstructure of polycrystalline materials for numerical analysis at mesoscopic level.

In order to provide a sufficiently accurate model to analyze the local stress state, the size of an RVE needs to be infinitesimal compared to the scale of the bulk material. On the other hand, the scale of RVE should be selected large enough compared to the size of microstructural features of the material so that it contains sufficient information of the microstructure. Suppose that $D^{\prime}$ is the scale of the bulk material, $L^{\prime}$ is the scale of the RVE and $d$ ' is the microstructure scale inside the RVE. The magnitudes of $D^{\prime}, L$ ' and $d$ 'should satisfy the relation: $d^{\prime} \ll L^{\prime} \ll D^{\prime}[68]$. Lemaitre [69] suggested that a suitable RVE is 0.1 $\times 0.1 \times 0.1 \mathrm{~mm}$ for metals. The RVE size also exerts a considerable influence on the macroscopic physical and mechanical properties of the materials. According to the FEM studies of Ren and Zhang [67], the errors of 574 cubic crystals' moduli were reported to be less than 5\% for a two-dimensional RVE with a size 20 times larger than the grain size [70].

\subsubsection{Voronoi tessellation}

Voronoi tessellation technique is always used to generate grain structure of a material model. A Voronoi tessellation is the partitioning of a cell structure with convex polygons 
or polyhedrons embedding a series of points and their domains of attraction. The mathematical definition of Voronoi tessellation is given as [71]: for an open set $\Omega \subseteq R^{N}$, the set $\left\{V_{i}\right\}_{i=1}^{k}$ is called a tessellation of $\Omega$ if $V_{i} \cap V_{i}=\emptyset$ for $\mathrm{i} \neq \mathrm{j}$ and $U_{i=1}^{k} \bar{V}_{i}=\bar{\Omega}$. Given a set of points $\left\{z_{i}\right\}_{i=1}^{k}$ belonging to $\bar{\Omega}$, the Voronoi region $z_{i}$ corresponding to the points is defined by:

$$
\widehat{V}_{\iota}=\left\{x \in \Omega|| x-z_{i}|<| x-z_{j} \mid \text { for } j=1, \ldots, k, j \neq i\right\} .
$$

The points $\left\{Z_{i}\right\}_{i=1}^{k}$ are called generators. The set $\left\{V_{i}\right\}_{i=1}^{k}$ is a Voronoi tessellation or Voronoi diagram of $\Omega$, and each $\widehat{V}_{l}$ is referred to as the Voronoi region corresponding to $z_{i}$. As schematically demonstrated in Figure 2.16, random generators $P_{i}$ are created in a 2D space. Each point belongs to only one partition $V\left(P_{i}\right)$ (Voronoi region) such that every vertex is closest to the corresponding generator.

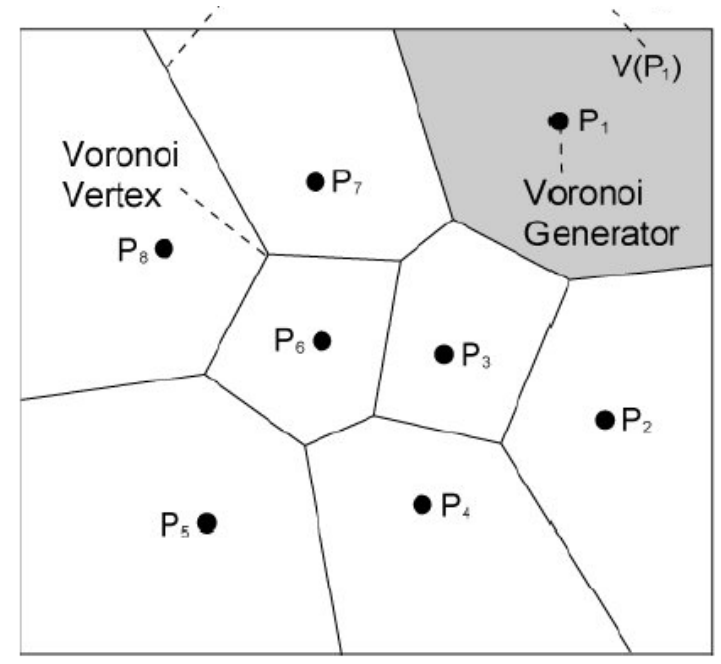

Figure 2.16 Graphical definition of Voronoi tessellation [72]. 
The concept of Voronoi tessellation provides a useful analysis tool in material science, especially to model random microstructures such as aggregates of grains in polycrystalline materials [70]. However, the topology of Voronoi tessellation is not exactly the same as the real material microstructures. For example, the shape of grains with rounded vertex in materials cannot be simulated by Voronoi cell which is constructed with only straight lines [68]. A two-dimensional Voronoi diagram constructed using Matlab codes is shown in Figure 2.17.

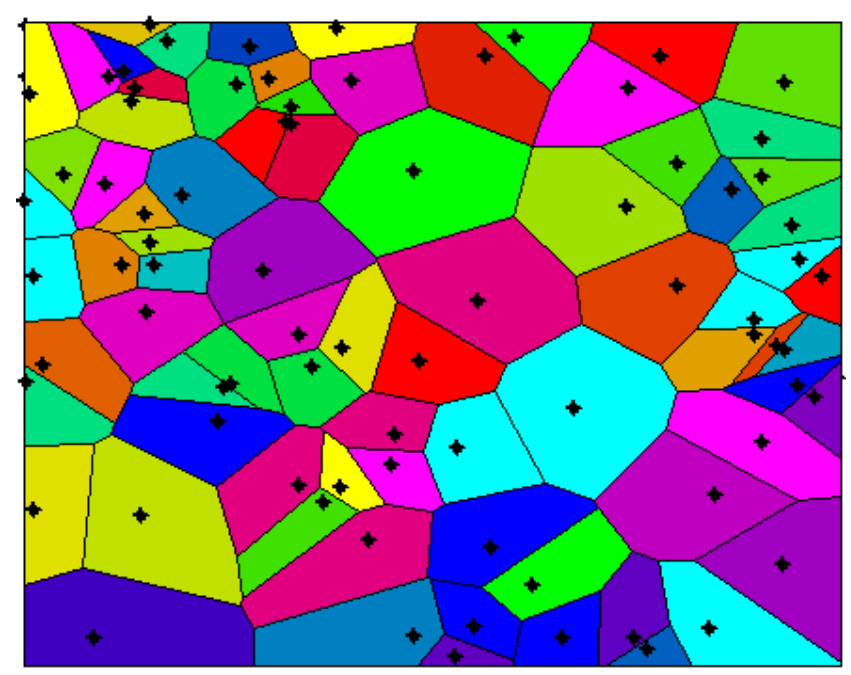

Figure 2. 17 A two-dimensional Voronoi diagram.

\subsection{Nickel-Based Superalloys}

Nickel-based superalloys are workhorse materials of high temperature components that find wide applications in gas turbine engines, owing to their microstructural stability, high strength, and good oxidation resistance [73]. Recently, a new alloy product, Haynes 282, 
is added to this family, as a candidate material for advanced ultra-supercritical (A-USC) steam turbine components. This material is chosen to be the subject material of this thesis study.

\subsubsection{Development history}

The history of nickel-based superalloys can be traced backed to the 1940s, when the demands of aircraft industry expanded [73]. At that time, Nimonic 75, as a milestone of nickel-based superalloys, was created for the Whittle's gas turbine engine in England [74]. Later on, development of nickel-based superalloys boomed, because Inconel alloys, Waspaloy, Rene alloys and Haynes alloys had been created for a wider range of applications in aerospace and marine gas turbine engines. The Haynes alloys, in particular, have been playing a major role in the aircraft gas turbine engine market due to their exceptional hightemperature mechanical properties combined with unexcelled oxidation resistance and improved thermal stability.

Despite that nickel-based superalloys show a distinctive advantage in high-temperature performance over other alloys, the machinability of these alloys is always a challenge [75]. As a results, a new wrought, gamma-prime strengthened nickel-based superalloy, designated as Haynes 282 (Ni-19Cr-10Co-8.5Mo-1.5Al-2.1Ti), has been developed and employed in hot-section components of gas turbine engines and advanced ultrasupercritical (A-USC) steam turbines. This alloy gains growing popularity because it 
possesses not only excellent high-temperature capability but also the outstanding weldability and fabricability. Consequently, Haynes 282 has been receiving an increasing attention in the turbine engine industry.

\subsubsection{Metallurgical properties}

Nickel-based superalloys contain major alloying elements including $\mathrm{Cr}, \mathrm{Co}, \mathrm{Al}$, Mo and other minor alloying elements such as $\mathrm{C}$ and $\mathrm{Ti}$. The high proportion of $\mathrm{Ni}$ content, usually from 30 to $70 \mathrm{wt} . \%$, provides an FCC nickel matrix which has high stability. $\mathrm{Cr}$ occupies 10 to 20 wt. $\%$ and confers outstanding corrosion and oxidation resistance. $\mathrm{Ti}$ and $\mathrm{Al}$ contribute to the formation of gamma prime $\gamma^{\prime}$ precipitates, which provide exceptional high-temperature strength [76][77]. Table 2.2 summarizes the effects of alloying elements in nickel-based superalloys [78]. Major phases present in the microstructure of nickelbased superalloys are gamma matrix $(\gamma)$, gamma prime $\left(\gamma^{\prime}\right)$, and carbides. Owing to their high strength, exceptional fatigue, creep and oxidation resistance at elevated temperatures and in complex loading conditions, nickel-based superalloys are popular materials for hot sections of jet engines. In addition, they also find wide applications in land-based and marine gas turbines, nuclear reactors, and chemical industry, etc. [75]. Some grades of nickel-based superalloys are Inconel 617, Inconel 718, Haynes 230 and Haynes 282. The tensile properties and physical properties of these alloys are given in Table 2.3 and Table 2.4, respectively. Since Haynes 282 alloy is selected as the subject material in this research, its fundamentals including chemical composition, microstructure and heat treatment are 
elaborated in following subsection.

Table 2. 2 Effects of alloying elements in nickel-based superalloys [79]

\begin{tabular}{|c|c|}
\hline Element & Effect \\
\hline Aluminum, Titanium & $\begin{array}{l}\mathrm{Al} \text { and } \mathrm{Ti} \text { form } \gamma^{\prime} \text { precipitates; Ti forms } \\
\mathrm{MC} \text { carbides (Mo- and Ti- rich) and } \mathrm{Al} \\
\text { forms protective oxide coating- } \mathrm{Al}_{2} \mathrm{O}_{3}\end{array}$ \\
\hline Chromium & $\begin{array}{l}\text { Hot corrosion and oxidation resistance, } \\
\text { and solid solution strengthening }\end{array}$ \\
\hline Molybdenum, Tungsten & $\begin{array}{l}\text { Solid solution strengthening and forms } \\
\qquad \mathrm{M}_{6} \mathrm{C} \text { carbides (Mo-rich) }\end{array}$ \\
\hline Cobalt & Increase $\gamma^{\prime}$ solvus temperature \\
\hline Carbon & Form primary and secondary carbides \\
\hline Niobium & Form delta-phase \\
\hline Boron, Zirconium & $\begin{array}{l}\text { Enhance rupture life; boron forms } \\
\text { borides in some alloys }\end{array}$ \\
\hline Tantalum & $\begin{array}{c}\text { Solid solution strengthening; form MC } \\
\text { carbides (Mo- and Ti-rich), and increase } \\
\text { oxidation resistance }\end{array}$ \\
\hline
\end{tabular}

(*M represents one or more metallic elements) 
Table 2. 3 Tensile properties of nickel-based superalloys [80] [76]

\begin{tabular}{|c|c|c|c|c|c|c|c|}
\hline Material & $\begin{array}{c}\text { Temperature } \\
\left({ }^{\circ} \mathrm{C}\right)\end{array}$ & Form & $\begin{array}{c}0.2 \% \\
\text { yield } \\
\text { strength } \\
(\mathrm{MPa})\end{array}$ & $\begin{array}{l}\text { Ultimate } \\
\text { tensile } \\
\text { strength } \\
(\mathrm{MPa})\end{array}$ & $\begin{array}{c}\text { Elongation } \\
(\%)\end{array}$ & $\begin{array}{l}\text { Young's } \\
\text { modulus } \\
(\mathrm{GPa})\end{array}$ & $\begin{array}{c}\text { Condition of } \\
\text { material }\end{array}$ \\
\hline \multirow{3}{*}{ Inconel } & 21 & Bar & 295 & 770 & 70 & 210 & \multirow{5}{*}{$1175^{\circ} \mathrm{C} / \mathrm{AC}$} \\
\hline & 540 & Bar & 200 & 590 & 68 & 176 & \\
\hline & 650 & Bar & 170 & 590 & 75 & 168 & \\
\hline \multirow{2}{*}{617} & 760 & Bar & 180 & 470 & 84 & 160 & \\
\hline & 870 & Bar & 195 & 310 & 118 & 150 & \\
\hline \multirow{5}{*}{$\begin{array}{c}\text { Inconel } \\
718\end{array}$} & 21 & Bar & 1185 & 1435 & 21 & 200 & \multirow{5}{*}{$\begin{array}{l}980^{\circ} \mathrm{C} / 1 \mathrm{~h} / \mathrm{AC}+ \\
720^{\circ} \mathrm{C} / 8 \mathrm{~h} / \mathrm{FC}+ \\
620^{\circ} \mathrm{C} / 8 \mathrm{~h} / \mathrm{AC}\end{array}$} \\
\hline & 540 & Bar & 1065 & 1275 & 18 & 171 & \\
\hline & 650 & Bar & 1020 & 1228 & 19 & 163 & \\
\hline & 760 & Bar & 740 & 950 & 25 & 154 & \\
\hline & 870 & Bar & 330 & 340 & 88 & 139 & \\
\hline \multirow{5}{*}{$\begin{array}{c}\text { Haynes } \\
230\end{array}$} & 21 & - & 390 & 870 & 48 & 211 & \multirow{5}{*}{$1230^{\circ} \mathrm{C} / \mathrm{AC}$} \\
\hline & 540 & -- & 275 & 720 & 56 & 184 & \\
\hline & 650 & - & 270 & 675 & 55 & 177 & \\
\hline & 760 & - & 285 & 575 & 46 & 171 & \\
\hline & 870 & -- & 225 & 385 & 59 & 164 & \\
\hline \multirow{5}{*}{$\begin{array}{c}\text { Haynes } \\
282\end{array}$} & RT & Sheet & 699 & 1132 & 30 & 217 & \multirow{5}{*}{$\begin{array}{l}1120^{\circ} \mathrm{C} / 1 \mathrm{~h} / \mathrm{WQ}+ \\
1010^{\circ} \mathrm{C} / 2 \mathrm{~h} / \mathrm{AC}+ \\
788^{\circ} \mathrm{C} / 8 \mathrm{~h} / \mathrm{AC}\end{array}$} \\
\hline & 600 & Sheet & 631 & 980 & 31 & 183 & \\
\hline & 700 & Sheet & 625 & 945 & 24 & 175 & \\
\hline & 800 & Sheet & 580 & 730 & 23 & 166 & \\
\hline & 900 & Sheet & 396 & 447 & 34 & 154 & \\
\hline
\end{tabular}

(RT represents room temperature; h stands for hour; AC, FC and WQ refer to air cooling, furnace cooling, water quenching, respectively.) 
Table 2. 4 Physical properties of nickel-based superalloys [80] [76]

\begin{tabular}{|c|c|c|c|c|c|}
\hline Material & $\begin{array}{c}\text { Temperature } \\
\left({ }^{\circ} \mathrm{C}\right)\end{array}$ & $\begin{array}{l}\text { Density } \\
\mathrm{g} / \mathrm{cm}^{3}\end{array}$ & $\begin{array}{c}\text { Specific } \\
\text { heat capacity } \\
(\mathrm{J} / \mathrm{kg} \cdot \mathrm{K})\end{array}$ & $\begin{array}{l}\text { Thermal } \\
\text { conductivity } \\
(\mathrm{W} / \mathrm{mK})\end{array}$ & $\begin{array}{c}\text { Mean coefficient of } \\
\text { thermal expansion } \\
\left(10^{-6} / \mathrm{K}\right)\end{array}$ \\
\hline \multirow{3}{*}{$\begin{array}{c}\text { Inconel } \\
617\end{array}$} & 21 & 8.36 & 420 & 13.6 & -- \\
\hline & 538 & 8.36 & 550 & 21.5 & 13.9 \\
\hline & 871 & 8.36 & 630 & 26.7 & 15.7 \\
\hline \multirow{3}{*}{$\begin{array}{c}\text { Inconel } \\
718\end{array}$} & 21 & 8.22 & 430 & 11.4 & -- \\
\hline & 538 & 8.22 & 560 & 19.6 & 14.4 \\
\hline & 871 & 8.22 & 645 & 24.9 & -- \\
\hline \multirow{3}{*}{$\begin{array}{c}\text { Haynes } \\
230\end{array}$} & 21 & 8.8 & 397 & 8.9 & -- \\
\hline & 538 & 8.8 & 473 & 18.4 & 14.0 \\
\hline & 871 & 8.8 & 595 & 24.4 & 15.2 \\
\hline \multirow{3}{*}{$\begin{array}{c}\text { Haynes } \\
282\end{array}$} & RT & 8.27 & 436 & 10.3 & -- \\
\hline & 500 & 8.27 & 563 & 20.5 & 13.5 \\
\hline & 900 & 8.27 & 668 & 28.9 & 15.9 \\
\hline
\end{tabular}

(RT represents room temperature.)

\subsubsection{Haynes 282 alloy}

Haynes 282 shares common metallurgical properties of nickel-based superalloys. The nominal composition of Haynes 282 alloy is detailed in Table 2.5. In this alloy, Ni occupies the highest weight percent (57 wt.\%) with $\mathrm{Cr}$ present at $20 \mathrm{wt} \%$. to provide outstanding resistance to both corrosion and oxidation. The presence of $10 \mathrm{wt} \%$ Co contributes to the regulation of gamma prime solvus while $\mathrm{Al}$ and $\mathrm{Ti}$ combined at about 3.6 wt.\% form 
gamma prime phases to harden the material. Moreover, Mo with $8.5 \mathrm{wt} . \%$, plays a critical role to improve creep strength. The standard heat treatment procedure for this alloy includes a solution treatment at $1121-1149{ }^{\circ} \mathrm{C}$, followed by two aging steps at $1010{ }^{\circ} \mathrm{C}$, for 2 hours (air cooling) and $780^{\circ} \mathrm{C}$ for 8 hours (air cooling). After a full heat treatment cycle, the microstructure of Haynes 282 contains $\gamma^{\prime}$ particles with primary MC carbides (Mo- and Ti-rich), Cr-rich $\mathrm{M}_{23} \mathrm{C}_{6}$ and Mo-rich $\mathrm{M}_{6} \mathrm{C}$ distributed along grain boundaries and inside grains, as shown in Figure 2.18 [81]. The unique combination of outstanding hightemperature mechanical properties and formability of Haynes 282 is achieved by balancing the fraction of $\gamma^{\prime}$ phase, which is found to improve properties while reducing fabrication difficulty of this alloy.

Haynes 282 alloy has been accepted by the engineering community as a reliable candidate for high-temperature structural applications in the engines of aircraft and land-based gas turbines [82]. Due to high temperature and stress conditions, these applications certainly make the claim for the properties of Haynes 282 alloy, particularly fatigue resistance. Consequently, a number of studies have been carried out to characterize the fatigue properties of Haynes 282 in recent years. Barat et al.[83] reported the influence of initial microstructure on the LCF behavior of Haynes 282 at elevated temperatures ranging from $650{ }^{\circ} \mathrm{C}$ to $760{ }^{\circ} \mathrm{C}$. Two types of microstructure with coarse and fine size distributions of $\gamma^{\prime}$ precipitates were achieved by different cooling rates (furnace cooling and air cooling). It is found that cyclic hardening or initial hardening followed by softening is dominant stress 
response for both microstructures and finer precipitates containing microstructure are prone to cyclically soften at high temperatures. Additionally, the thermal fatigue of Haynes 282 is found to undergo with various deformation modes including planar and nonplanar dislocation activities, which are governed by test temperature and strain amplitude. Precipitate looping and bypassing are main deformation mechanisms in lower temperature and higher strain amplitude condition while precipitate shearing dominates at lower temperature and lower strain amplitude. He et al. [84] investigated the room-temperature and high-temperature LCF behavior of Haynes 282 from a large forged ingot. The research revealed that the primary failure mode of Haynes 282 was represented by transgranular fatigue cracks. It showed better resistance to $\mathrm{LCF}$ at $750^{\circ} \mathrm{C}$, compared to other nickel-based superalloys. In a room-temperature LCF study made by Buckson and Ojo [85], Haynes 282 experienced initial hardening followed by cyclic softening. The initial cyclic hardening in the early stage of the fatigue test was caused by an increase in the density of slip bands. The LCF fatigue data were also characterized by the Coffin-Manson equation and the cyclic deformation parameters indicated strong fatigue resistance of Haynes 282 at room temperature. Yang et al. [86] studied the HCF behavior of Haynes 282 at room temperature and elevated temperatures $\left(700^{\circ} \mathrm{C}\right.$ and $\left.760^{\circ} \mathrm{C}\right)$. The crack initiation was found to take place in the surface stratum or subsurface stratum. The results also showed that the HCF strength of Haynes 282 decreased as temperature increased due to synergetic effect of precipitate cutting and Orowan looping in fatigue at high temperatures. 
However, aforementioned studies in the fatigue failure of Haynes 282 alloy highly relied on experimental testing, which caused a large amount of cost and time. Insufficient focus has been placed on the assessment of fatigue life of this alloy with different couplings of temperature and loading condition in a more efficient and economical manner. Therefore, the present study intends to contribute to the fatigue crack nucleation life prediction of Haynes 282 by the combination of computational modeling and numerical analysis.

Table 2. 5 Nominal composition (wt.\%) of Haynes 282 alloy [79]

\begin{tabular}{|c|c|c|c|c|c|c|c|c|c|c|c|c|}
\hline Alloy & $\mathrm{Ni}$ & $\mathrm{Cr}$ & $\mathrm{Co}$ & $\mathrm{Mo}$ & $\mathrm{Ti}$ & $\mathrm{Al}$ & $\mathrm{Fe}$ & $\mathrm{Mn}$ & $\mathrm{Si}$ & $\mathrm{C}$ & $\mathrm{B}$ & Others \\
\hline 282 & $57 \mathrm{a}$ & 20 & 10 & 8.5 & 2.1 & 1.5 & $1.5^{*}$ & $0.3^{*}$ & $0.15^{*}$ & 0.06 & 0.005 & -- \\
\hline
\end{tabular}

(Note: "a" refers to balance and “*” stands for at most.)

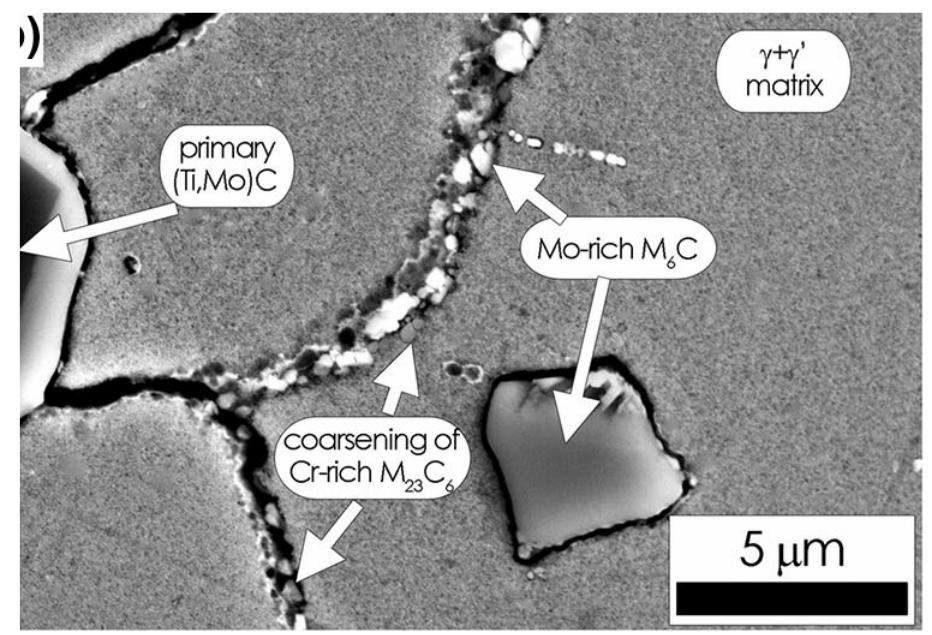

Figure 2. 18 Scanning Electron Microscope (SEM) microstructure of Haynes 282 after standard aging treatment [81]. 


\section{Chapter 3 Fatigue Life Prediction with the TMW Model}

As discussed in previous chapters, traditionally, the fatigue lives of engineering materials are determined by performing various fatigues tests, either in stress or in strain-controlled mode, which results in high costs in time and money, also prolonging the development cycle in material and component design for mechanical/structural systems. Therefore, computational fatigue design has become more attractive in fatigue studies of materials. In this chapter, an analytical model developed by Wu et al. [8][9], named the TMW model, is further studied with eight different engineering materials which are all commonly employed on mechanical/structural components under fatigue loading conditions. The TMW model will be introduced in more detail in section 3.1, with respect to the strainbased and stress-based fatigue life prediction formulas as well as the model parameters involved. In section 3.2, both strain-based and stress-based formulas are checked against fatigue data or Coffin-Manson-Basquin curves for the selected eight alloys including aluminum alloys, nickel alloys, steels and titanium alloys. Finally, in section 3.3, the relationship between the surface roughness factor $R_{S}$ in the TMW model and the profile roughness parameter $R_{a}$ is developed based on the available fatigue data of aluminum alloy and medium-carbon steel in literature, which illustrates an engineering approach to determining $R_{S}$ for fatigue design of structures with different surface finish. The significance of this work is to expedite the engineering application of the TMW model to save material coupon testing and money, and furthermore to predict the fatigue properties 
of new materials since they emerge from new manufacturing processes such as additive manufacturing and high entropy alloy design, etc.

\subsection{The TMW Model}

\subsubsection{Fatigue crack nucleation life}

Currently, the fatigue behavior of most existing structural materials has been characterized experimentally in terms of e- $\mathrm{N}$ curve (a plot of the relation between total strain and the number of cycles to failure) or S-N curve (a plot of the magnitude of an alternating stress versus the number of cycles to failure), as correlations of fatigue test data. As discussed in Chapter 2, the TMW model [8][9] promises prediction of fatigue crack nucleation life with material properties including shear modulus $\mu$, Poisson's ratio $v$, Burgers vector $b_{v}$, surface energy $w_{s}$ and the lattice resistance $\sigma_{0}$, as:

$$
N_{c}=\frac{8(1-v) R_{s} w_{s}}{3 \mu b_{v}} \frac{1}{\Delta \varepsilon_{p}{ }^{2}}
$$

or

$$
N_{c}=\frac{6 \mu R_{s} w_{s}}{(1-v)\left(\Delta \sigma-2 \sigma_{0}\right)^{2} b_{v}}
$$

where $\Delta \varepsilon_{p}$ is the cyclic plastic strain range, and $\Delta \sigma$ the cyclic stress range, $R_{S}$ is the surface roughness factor.

For most existing structural materials, the Young's modulus, Poisson's ratio, Burgers vector, and surface energy are either known or can be determined independently using physical 
test methods. However, the surface roughness factor $R_{S}$ and the minimum lattice resistance $\sigma_{0}$ are not exactly known, due to variations of manufacturing processes. For low cycle fatigue (LCF), $\mathrm{Eq}$ (3.1) has been validated against a number of pure metals and alloys with $R_{S} \approx 1 / 3$ as determined by comparison of fatigue lives of machined coupons to electropolished $\left(R_{S}=1\right)$ coupons. Later in section 3.2, validation of the TMW model, viz. Eq (3.1) and (3.2), is extended to the entire fatigue range including high cycle fatigue (HCF), along with the determination of the surface roughness factor $R_{S}$ and the minimum lattice resistance $\sigma_{0}$.

\subsubsection{Fatigue curves}

The fatigue behavior of most engineering materials is often characterized as strain-life and stress-life relations. Such relationships are used to be established via large amounts of coupon testing, which results in high costs of materials, time, and money. The strain-life relationship is useful for fatigue design of component structures with stress concentration features where the cyclic damage is associated with local plasticity and the process is basically strain-controlled. In this case, Coffin-Manson equation is always used to describe the correlation between plastic strain and fatigue life:

$$
\frac{\Delta \varepsilon_{p}}{2}=\varepsilon_{f}{ }^{\prime}\left(2 N_{f}\right)^{c}
$$

where $\Delta \varepsilon_{p}$ is plastic strain range, $\varepsilon_{f}{ }^{\prime}$ is fatigue ductility coefficient and $c$ is fatigue ductility exponent. 
The stress-life relation is typically used for the fatigue design of component structures under stress-controlled loading. This is extremely practical in HCF region where component structures are elastically deformed at macroscopic level in fatigue failure. Basquin equation is often employed as a stress-life approach:

$$
\frac{\Delta \varepsilon_{e}}{2}=\frac{\Delta \sigma}{2 E}=\frac{\sigma_{f^{\prime}}}{E}\left(2 N_{f}\right)^{b}
$$

where $\Delta \varepsilon_{e}$ is elastic strain range, $\Delta \varepsilon$ is total strain range, $E$ is the young's modulus, $\sigma_{f}{ }^{\prime}$ is the fatigue strength coefficient and $b$ is fatigue strength exponent. Then the total strain-life equation, which is also known as Coffin-Manson-Basquin equation, is given as the combination of Eq (3.3) and (3.4):

$$
\frac{\Delta \varepsilon}{2}=\frac{\sigma_{f^{\prime}}}{E}\left(2 N_{f}\right)^{b}+\varepsilon_{f}{ }^{\prime}\left(2 N_{f}\right)^{c}
$$

The equations are used in this research to estimate the fatigue lives of the selected materials except Haynes 282 alloy, which represent the experimental results to compare with the predictions from the TMW model. The fatigue data of selected materials except Haynes 282 alloy were fitted by Eq (3.3) to (3.5) for given loading conditions [87] [88] [89] [90] [85]. The cyclic life parameters related to Coffin-Manson-Basquin equation are analyzed in next section. For Haynes 282 alloy, the experimental fatigue data are directly used for the comparison, because an error in the data fitting was identified in the work of Buckson and Ojo [85]. 


\subsection{Fatigue Life Analysis}

\subsubsection{Materials and properties}

Eight engineering alloys ranging from aluminum alloys, nickel alloys, titanium alloys are selected in this study for fatigue life analysis (due to the limited scope in time, the selection cannot be exhaustive for all existing materials), as described below. These alloys find popular applications in aerospace, transportation and energy industries.

The first selected material is 7075-T6 aluminum alloy, which contains $5.6-6.1 \mathrm{wt} . \%$ zinc, $2.1-2.9$ wt. $\%$ magnesium, $1.2-2$ wt. $\%$ copper, and minor amount ( $<0.5$ wt. $\%)$ of other elements such as silicon, iron, manganese, titanium, chromium, etc. The T6 represents a temper treatment condition which allows the plate in its peak-aged condition, i.e., strengthened mostly by Guinier-Preston zones ( $\mathrm{Zn}-\mathrm{Mg}$ clusters) or metastable $\eta^{\prime}\left(\mathrm{MgZn}_{2}\right)$ precipitates. The detailed chemical composition of this alloy is given in Table 3.1; the monotonic and cyclic properties of the alloy are given in Table 3.2 and Table 3.3, respectively [87]. This alloy has a wide range of application in aerospace, owing to its high strength, toughness, and good ductility and fatigue resistance. The cyclic life parameters $\sigma_{f}{ }^{\prime}, b, \varepsilon_{f}{ }^{\prime}$ and $c$ are empirical constants of Coffin-Manson and Basquin relations [7] which are established by fitting to large amounts of fatigue data (the same for the other alloys selected).

The second selected material is 2024-T3 aluminum alloy, which contains $3.8-4.9$ wt.\% 
copper, $0.3-0.9$ wt.\% manganese, $1.2-1.8 \mathrm{wt} . \%$ magnesium, and minor amount $(<0.5$ wt.\%) of other elements such as silicon, nickel, zinc, lead, bismuth, etc. The T3 represents a temper condition which allows the sheet in its peak-aged condition, i.e., strengthened mostly by $S(\mathrm{~A} 12 \mathrm{CuMg})$ precipitates and $\theta^{\prime}\left(\mathrm{CuAl}_{2}\right)$ precipitates. The detailed chemical composition of this alloy is given in Table 3.1; the monotonic and cyclic properties of the alloy are given in Table 3.2 and Table 3.3, respectively [3]. This alloy is often employed in aircraft structural applications owing to its high strength and fatigue resistance.

The third selected material is SAE 1020 alloy steel, which is a low-carbon steel containing $0.3-0.6$ wt. $\%$ magnesium, $0.17-0.23$ wt. $\%$ carbon, and minor amount $(<0.5$ wt. $\%)$ of other elements including phosphorous and sulfur. This alloy has good machinability, high strength, high ductility and good weldability and is often used for common engineering and machinery parts and components such as shafts and camshafts, gudgeon pins, ratchets, light duty gears and spindles. The detailed chemical composition of this alloy is given in Table 3.1; the monotonic and cyclic properties of the alloy are reported in Table 3.2 and Table 3.3 [88], respectively.

The fourth selected material is SAE 4340 steel, which is a low-alloy steel containing 1.83 wt. $\%$ nickel, $0.7-0.9$ wt. $\%$ chromium, 0.7 wt. $\%$ manganese and minor amount $<0.5$ wt. $\%$ ) of other elements such as carbon, silicon, sulfur, phosphorus, etc. This alloy is mainly used for power transmission gears and shafts, aircraft landing gear, and other structural parts. The detailed chemical composition of this alloy is given in Table 3.1; the monotonic and 
cyclic properties of the alloy are given in Table 3.2 and Table 3.3, respectively [87].

The fifth selected material is Ti-6Al-4V alloy, which is an $\alpha+\beta$ alloy with 6 wt.\% aluminum as the $\alpha$ phase stabilizer and 4 wt. $\%$ vanadium as the $\beta$ phase stabilizer plus minor amount $(<0.5$ wt.\%) of oxygen and iron. This alloy finds many applications in aerospace and biomechanical industries due to its high strength, good fatigue resistance and outstanding corrosion resistance. The detailed chemical composition and the monotonic and cyclic properties of this alloy are given in Table 3.1 to Table 3.3, respectively [89].

The sixth selected material is Inconel 617 alloy, which is a solid-solution strengthened nickel-base alloy with 20 - 24 wt.\% chromium, 10 to 15 wt.\% cobalt, 8 to 10 wt.\% molybdenum, $\sim 3$ wt. $\%$ iron, 0.8 to 1.5 wt. $\%$ aluminum, and minor amount $(<0.5$ wt. $\%)$ of other elements such as iron, manganese, silicon, etc. This alloy is an attractive material for critical structural components such as combustion cans, ducting and transition liner in aircraft and land-based gas turbines, owing to its combination of high strength and high temperature oxidation resistance. The detailed chemical composition, the monotonic and cyclic properties of this alloy are given in Table 3.1 to Table 3.3, respectively [90].

The seventh selected material is Inconel 718 alloy, which is a precipitation-hardenable nickel-chromium alloy containing $17-21$ wt. $\%$ chromium, 17 wt.\% iron, 4.75 to 5.5 wt. $\%$ niobium, 2.8 to 3.3 wt.\% molybdenum and other minor elements such as cobalt and 
titanium, etc. This alloy finds many applications in both aero and land-based gas turbine engines due its high strength, excellent heat resistance and good weldability. The detailed chemical composition, the monotonic and cyclic properties of this alloy are given in Table 3.1 to Table 3.3 , respectively.

The last selected alloy is Haynes 282, which is the subject material of the present study. The metallurgical properties as well as applications of this alloy have already been introduced in Chapter 2. The detailed chemical composition and monotonic properties of this alloy are given in Table 3.1 and 3.2. For this material, the fatigue life predictions by the TMW model are directly compared with experimental data from literature [85].

Table 3. 1 Chemical compositions of selected materials [91]

\begin{tabular}{|c|c|c|c|c|c|}
\hline \multirow{2}{*}{ Material } & \multicolumn{5}{|c|}{ Composition (wt.\%) } \\
\hline \multirow{3}{*}{ Al7075-T6 } & $\mathrm{Al}$ & $\mathrm{Zn}$ & $\mathrm{Mg}$ & $\mathrm{Cu}$ & $\mathrm{Fe}, \mathrm{Si}, \mathrm{Mn}$, etc \\
\cline { 2 - 6 } & $87.1-91.4$ & $5.1-6.1$ & $2.1-2.9$ & $1.2-2$ & $\mathrm{Bal}$ \\
\hline \multirow{3}{*}{ Al2024-T3 } & $\mathrm{Al}$ & $\mathrm{Cu}$ & $\mathrm{Mg}$ & $\mathrm{Mn}$ & $\mathrm{Ni}, \mathrm{Si}, \mathrm{Zn}$, etc \\
\cline { 2 - 6 } & $90.7-94.7$ & $3.8-4.9$ & $1.2-1.8$ & $0.3-0.9$ & $\mathrm{Bal}$ \\
\hline \multirow{3}{*}{ SAE 1020 } & $\mathrm{Fe}$ & $\mathrm{Mn}$ & $\mathrm{C}$ & $\mathrm{S}$ & $\mathrm{P}$ \\
\cline { 2 - 7 } & $99.08-99.53$ & $0.3-0.6$ & $0.17-0.23$ & $\leq 0.05$ & $\leq 0.04$ \\
\hline \multirow{3}{*}{ SAE 4340 } & $\mathrm{Fe}$ & $\mathrm{Ni}$ & $\mathrm{Mn}$ & $\mathrm{Cr}$ & $\mathrm{Mo}, \mathrm{C}, \mathrm{Si}$, etc \\
\cline { 2 - 6 } & 96 & 1.83 & 0.7 & $0.7-0.9$ & $\mathrm{Bal}$ \\
\hline & $\mathrm{Ti}$ & $\mathrm{Al}$ & $\mathrm{V}$ & $\mathrm{Fe}$ & $\mathrm{O}$ \\
\hline
\end{tabular}




\begin{tabular}{|c|c|c|c|c|c|}
\hline Ti-6Al-4V & 90 & 6 & 4 & $\leq 0.25$ & $\leq 0.2$ \\
\hline \multirow{3}{*}{ Inconel 617} & $\mathrm{Ni}$ & $\mathrm{Cr}$ & $\mathrm{Co}$ & $\mathrm{Mo}$ & $\mathrm{Fe}, \mathrm{Mn}, \mathrm{Si}$, etc \\
\cline { 2 - 6 } & $\geq 44.5$ & $20-24$ & $10-15$ & $8-10$ & $\mathrm{Bal}$ \\
\hline \multirow{3}{*}{ Inconel 718 } & $\mathrm{Ni}$ & $\mathrm{Cr}$ & $\mathrm{Fe}$ & $\mathrm{Nb}$ & $\mathrm{Mo}, \mathrm{Co}, \mathrm{Ti}$, etc \\
\cline { 2 - 6 } & $50-55$ & $17-21$ & 17 & $4.75-5.50$ & $\mathrm{Bal}$ \\
\hline \multirow{3}{*}{ Haynes 282 } & $\mathrm{Ni}$ & $\mathrm{Cr}$ & $\mathrm{Co}$ & $\mathrm{Mo}$ & $\mathrm{Ti}, \mathrm{Al}, \mathrm{Fe}$, etc \\
\cline { 2 - 6 } & 57 & 20 & 10 & 8.5 & $\mathrm{Bal}$ \\
\hline
\end{tabular}

Table 3. 2 Monotonic mechanical properties of selected materials [87] [88] [89] [90] [85]

\begin{tabular}{|c|c|c|c|c|}
\hline Material & $\begin{array}{c}\text { Young's } \\
\text { modulusE } \\
(\mathrm{GPa})\end{array}$ & $\begin{array}{c}\text { Yield stress } \\
\sigma_{\mathrm{ys}}(\mathrm{MPa})\end{array}$ & $\begin{array}{c}\text { Tensile } \\
\text { strength } \sigma_{u} \\
(\mathrm{MPa})\end{array}$ & $\begin{array}{c}\text { Elongation } \\
\text { EI (\%) }\end{array}$ \\
\hline $\mathrm{Al} 7075-\mathrm{T} 6$ & 71 & 468 & 572 & 11 \\
\hline $\mathrm{Al}$ 2024-T3 & 70 & 403 & 483 & 11 \\
\hline SAE 1020 & 205 & 285 & 491 & 36.50 \\
\hline SAE 4340 & 200 & 889 & 1110 & 13.2 \\
\hline Ti-6Al-4V & 117 & 1185 & 1200 & 10 \\
\hline Inconel 617 & 214.4 & 346 & 811.1 & 62 \\
\hline Inconel 718 & 209 & 1160 & 1200 & 25 \\
\hline Haynes 282 & 217 & 715 & 1132 & 30 \\
\hline
\end{tabular}

Table 3. 3 Cyclic properties of selected materials [87] [88] [89] [90] [85]

\begin{tabular}{|c|c|c|c|c|c|c|}
\hline Material & $\mathrm{K}^{\prime}(\mathrm{MPa})$ & $\mathrm{n}^{\prime}$ & $\sigma_{\mathrm{f}}{ }^{\prime}(\mathrm{MPa})$ & $\mathrm{b}$ & $\varepsilon_{\mathrm{f}}{ }^{\prime}$ & $\mathrm{c}$ \\
\hline A1 7075-T6 & 780.64 & 0.088 & 780.64 & -0.045 & 0.19 & -0.52 \\
\hline Al 2024-T3 & 751.1 & 0.1 & 737.7 & -0.081 & 0.3 & -0.6 \\
\hline SAE 1020 & 941 & 0.18 & 815 & -0.114 & 0.25 & -0.53 \\
\hline SAE 4340 & 1910 & 0.123 & 1879 & -0.0895 & 0.64 & -0.636 \\
\hline
\end{tabular}




\begin{tabular}{|c|c|c|c|c|c|c|}
\hline Ti-6Al-4V & 1772 & 0.11 & 2030 & -0.104 & 0.84 & -0.69 \\
\hline Inconel 617 & 1910 & 0.118 & 1519.55 & -0.104 & 0.501 & -0.567 \\
\hline Inconel 718 & 1564 & 0.0681 & 3950 & -0.151 & 1.5 & -0.761 \\
\hline
\end{tabular}

\subsubsection{Parameters of the TMW model}

The TMW model uses only material physical properties, the determination of which is detailed in this section. Since the procedures of the parameter determination are essentially the same for all selected materials, as example for demostration, only the one for Al 7075T6 is delineated below.

\section{1) Shear modulus}

Elastic modulus (the Young's modulus) is a basic material constant that describes an object or substance's resistance to elastic deformation along the loading direction when a stress is applied on it. It is often measured from the linear portion of stress-strain relationship for engineering materials [92]. For monolithic materials, it can also be computed using ab initio density functional theory [93], based on the material's atomistic structural configuration. Shear modulus describes the response of a solid to shear stress under plastic regime. For an isotropic material, the shear modulus $\mu$ is converted from the Young's modulus $E$ by the relationship, $\mu=\frac{E}{2(1+v)}$ where $E$ is young's modulus and $v$ is poisson's ratio [94]. For Al 7075-T6, $\mu=26.89 \mathrm{GPa}$. 


\section{2) Burgers vector}

The Burgers vector $b_{v}$ represents the magnitude and direction of lattice distortion caused by dislocation in a crystal lattice [95]. The magnitude of Burger's vector is expressed by $\left\|b_{v}\right\|=\left(\frac{e}{2}\right) \sqrt{h^{2}+k^{2}+l^{2}}$ where $e$ is the unit cell edge length of the crystal and $h, k, l$ are the $x, y$ and $z$ components of the Burger's vector [96], respectively. For Al 7075-T6, it is assumed to be the same as that of aluminum from literature [97], $2.86 \times 10^{-10} \mathrm{~m}$;

\section{3) Surface energy}

The surface energy $w_{s}$ quantifies the disruption of intermolecular bonds that occurs when a surface is created. It can also be defined as the work required to cut the bulk material and lead to crack formation [98]. The surface energy is usually measured through contact angle experiment by a contact angle meter using liquids such as water and diiodomethane as measuring medium. The surface energy can be determined from the measured contact angle data and the surface tension of liquids [99]. In addition, the surface energy can be calculated using $a b$ initio density functional theory [100]. As introduced in Chapter 2, the surface energy $w_{s}$ in this study is estimated by [41]:

$$
w_{s}=w_{s}\left(T_{m}\right)+\phi(T) \frac{R T_{m}}{A}
$$

where $w_{s}\left(T_{m}\right)$ is the surface energy at the melting temperature, $A$ is the surface area per mole of surface atoms, $\phi(T)$ is a temperature-dependent parameter and $R$ is the Boltzmann constant. In this case, at room temperature $\left(20^{\circ} \mathrm{C}\right)$, the homologous temperature (thermodynamic temperature of a material as a fraction of its melting point temperature 
using the Kelvin scale) is $\sim 0.32$ (the melting temperature of Al $7075-\mathrm{T} 6$ is $635^{\circ} \mathrm{C}$, according to MatWeb database [91]). Then according to the studies of Tyson [41], the parameter $\phi$ can be determined to be 0.82 and surface energy $w_{s}$ can be calculated as: $w_{s}=w_{s}\left(T_{m}\right)+\phi(T) \frac{R T_{m}}{A}=1.02+0.123 \times 0.82 \approx 1.121 \mathrm{~J} / \mathrm{m}^{2}$.

\section{4) Lattice resistance}

Lattice resistance is one of the most fundamental resistances to dislocation motion in a pure crystalline material in a temperature range where diffusion plays no role. At first, it arises from the Peierls-Nabarro (PN) resistance, which is the force pulsing distortions of the dislocation core as it moves through the discrete lattice [101]. In complex engineering alloys, the presence of solute atoms, precipitates and grain boundaries will exert a back stress that effectively raises the lattice resistance to dislocation motion on the plane intersecting these obstacles [1]. For complex engineering alloys, it is difficult to measure the minimum lattice resistance at commencing dislocation motion, because the total deformation at this stage is mainly elastic. The stress-based Eq (3.2) considers the role of lattice resistance $\sigma_{0}$, in addition to the effect of other common properties as in the strainbased Eq (3.1). Physically, the stress-based fatigue life should correspond to the stress level at which incipient formation of slip bands occurs, but this needs extensive metallurgical examinations to be performed on the coupons tested under different stress levels to ascertain, which involves massive labor and is impractical for engineering fatigue design.

Therefore, in the present study, $\sigma_{0}$ is determined by fitting Eq (3.2) to the experimental 
stress level close to the fatigue life of $1 \times 10^{7}$. For Al 7075-T6, it is thus determined to be $377 \mathrm{MPa}$. For simplicity, the parameter $R_{S}$ is assumed to be 1 for HCF (The premise is that the effect of roughness assisting crack nucleation is different when the deformation is macroscopically elastic or plastic, more analysis and discussion will be given in section 3.3). The material property values used in $\mathrm{Eq}$ (3.1) and $\mathrm{Eq}$ (3.2) for all the materials selected are summarized in Table 3.4 .

Table 3. 4 Calculated fatigue coefficients for selected materials

\begin{tabular}{|c|c|c|c|c|c|c|}
\hline Material & $v$ & $\mu(\mathrm{GPa})$ & $\mathrm{w}_{\mathrm{s}}\left(\mathrm{J} / \mathrm{m}^{2}\right)$ & $\sigma_{0}(\mathrm{MPa})$ & $\mathrm{R}_{\mathrm{s}}$ & $\mathrm{b}_{\mathrm{v}}\left(10^{-10} \mathrm{~m}\right)$ \\
\hline $\mathrm{Al} 7075-\mathrm{T} 6$ & 0.32 & 26.89 & 1.121 & 377 & $1 / 3$ or 1 & 2.86 \\
\hline $\mathrm{Al}$ 2024-T3 & 0.32 & 26.52 & 1.112 & 225 & $1 / 3$ or 1 & 2.86 \\
\hline SAE 1020 & 0.29 & 79.45 & 2.373 & 116 & $1 / 3$ or 1 & 2.48 \\
\hline SAE 4340 & 0.3 & 76.92 & 2.388 & 500 & $1 / 3$ or 1 & 2.48 \\
\hline Ti-6Al-4V & 0.34 & 45 & 1.970 & 495 & $1 / 3$ or 1 & 3.21 \\
\hline Inconel 617 & 0.34 & 82.46 & 2.350 & 298 & $1 / 3$ or 1 & 2.48 \\
\hline Inconel 718 & 0.33 & 78.57 & 2.350 & 455 & $1 / 3$ or 1 & 2.48 \\
\hline Haynes 282 & 0.319 & 82.26 & 2.350 & 290 & $1 / 3$ or 1 & 2.48 \\
\hline
\end{tabular}

\subsubsection{Fatigue curves for $\mathrm{LCF}$ and $\mathrm{HCF}$}

Once the required material properties for the TMW model are determined, as demonstrated in the above section, the fatigue lives of the selected materials can be easily calculated using the strain/stress-based formula, Eq (3.1) or Eq (3.2). Naturally, for LCF with appreciable macroscopic plastic strain $\left(>10^{-3}\right), \mathrm{Eq}(3.1)$ is the choice; and for HCF, where 
macroscopic plastic strain is not discernable, Eq (3.2) is applicable, because the stress can be accurately determined from microscopic stress-strain analysis. The reason why Eq (3.1) and $\mathrm{Eq}(3.2)$ are applied to different regions is that above the $0.2 \%$ offset the material yields with extensive dislocation multiplication, forming a dislocation network within the material. Therefore, the stress-strain relation of the TMW model based on the formation of dislocation pile-up only on the maximum slip plane is not realistic for real materials. Instead, the Ramberg-Osgood equation [27] represents the true stress-strain relationship for the materials.

Figure 3.1 shows the comparison of $\mathrm{Eq}(3.1)\left(R_{s} \approx 1 / 3\right)$ with the Coffin-Manson relation for Al 7075-T6. The agreement between Eq (3.1) and the experimental data regression line appears good, especially at plastic strain amplitude above 0.001 . The discrepancy between $\mathrm{Eq}$ (3.1) and the Coffin-Manson relation is not totally unexpected. Since the "experimental behavior" below 0.001 is actually the extrapolation of the Coffin-Manson correlation for large strain fatigue data, any small variation in the slope of linear regression would result in large deviation in the extrapolated behavior. It is well known that the slope and intercept of linear regression in a log-log scale depend on the dataset (the number of tests and scatter). Even though Eq (3.1) does not evaluate scatter directly, by comparison it provides a baseline to which the effects of other factors, e.g., surface finish, can be evaluated. 


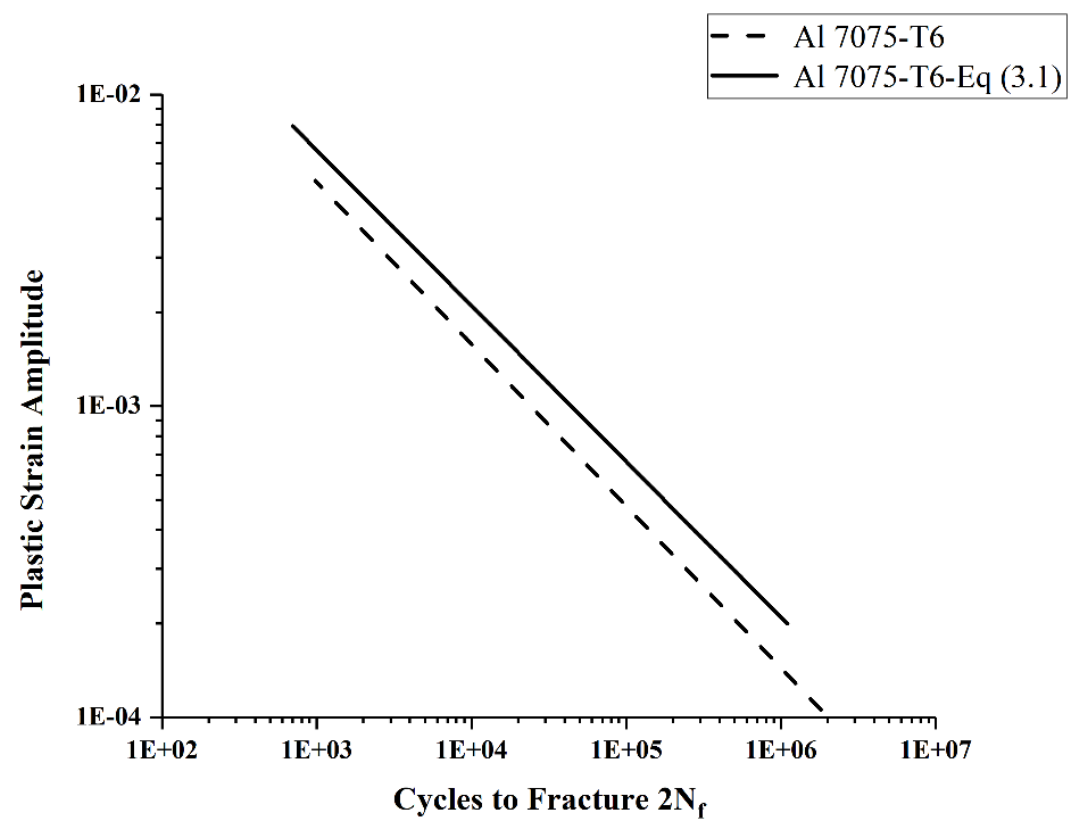

Figure 3. 1 Comparison between theoretical prediction of Eq (3.1) and Coffin-Manson curve for Al 7075-T6.

Figure 3.2 shows the comparison of Eq (3.2) and Basquin equation, Eq (3.4), for $\mathrm{HCF}$ below the yield strength, and the predictions of Eq (3.2) well coincide with the Basquin curve in the HCF region $\left(N_{f}>10^{5}\right.$ cycles $)$ with a typical scatter factor of 2. 


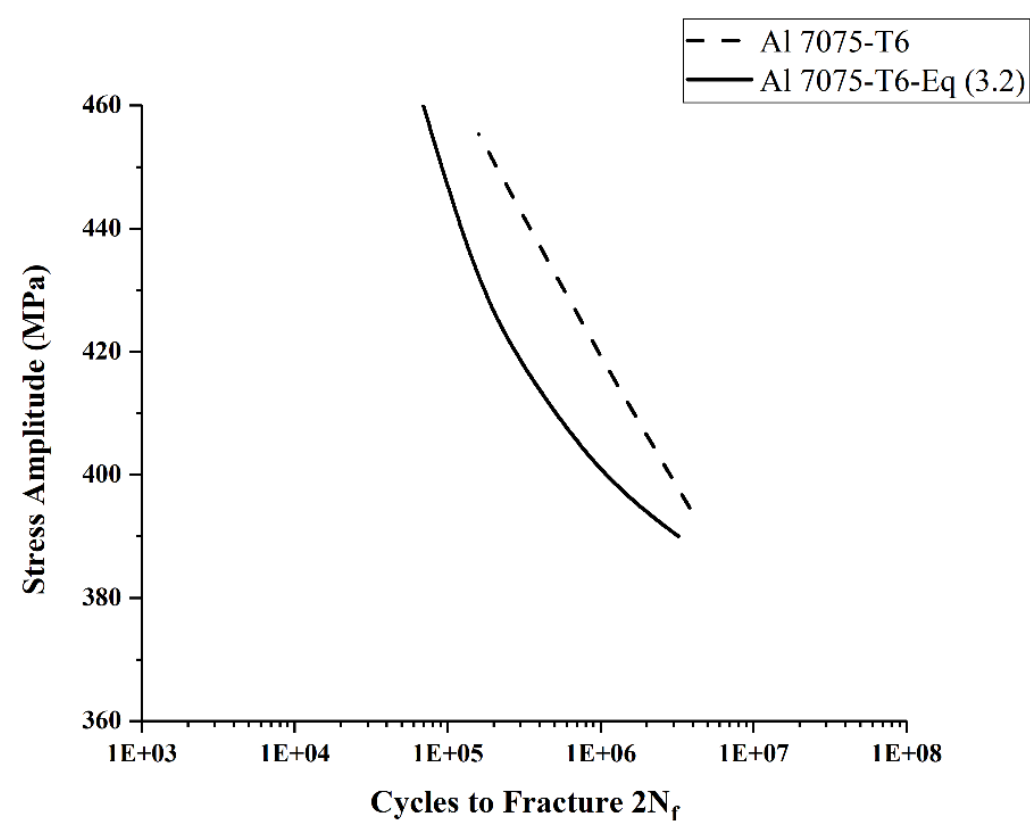

Figure 3. 2 Comparison between theoretical prediction of Eq (3.2) and Basquin curve for Al 7075-T6.

Finally, the predictions of Eq (3.1) for the LCF region (plastic strain amplitude greater than 0.001) and predictions of Eq (3.2) for the HCF region are illustrated together in a total strain-life plot in Figure 3.3, in comparison with the combined Coffin-Manson-Basquin curve. The overall validity of the TMW model for Al 7075-T6 has been demonstrated as the prediction is very close the experimental curve, given the assumed roughness condition. Particularly, Eq (3.1) is effective for the LCF region (plastic strain above 0.001); while for the HCF region where deformation is macroscopically elastic below the yield point, Eq (3.2) is effective once $\sigma_{0}$ is calibrated with one fatigue test. The TMW model has shown to be capable of predicting the fatigue life of Al 7075-T6 over the entire LCF + HCF region. 


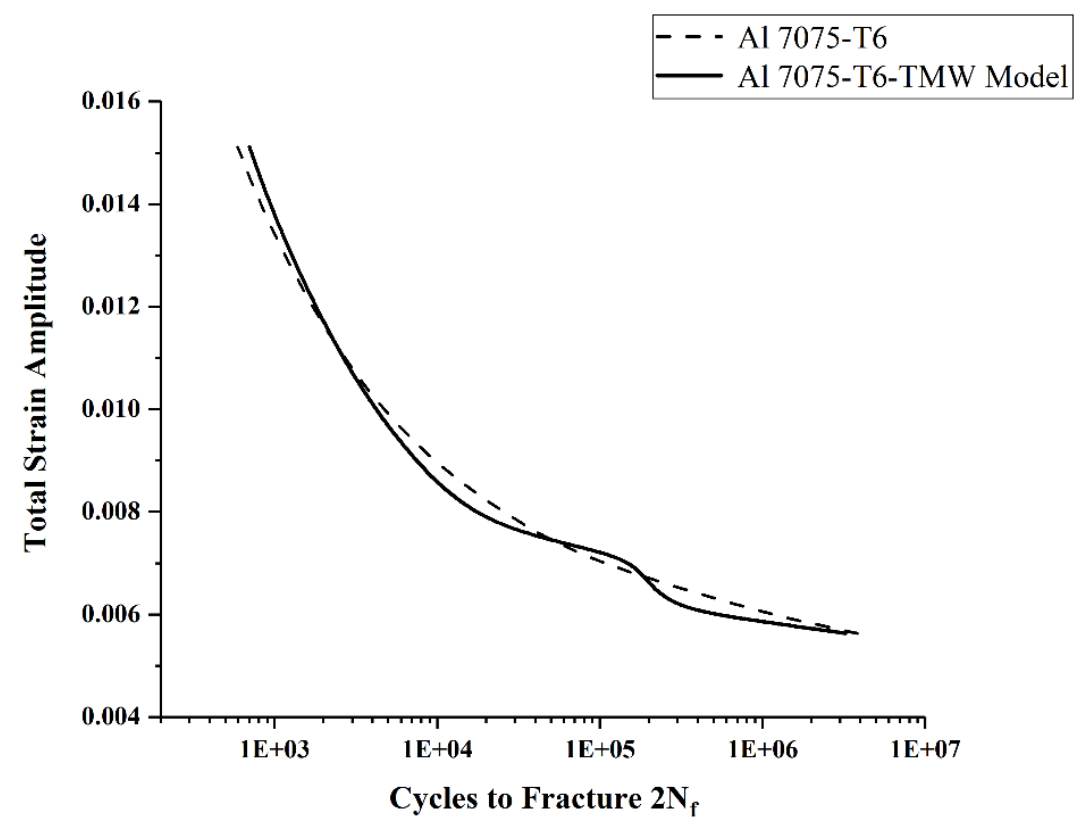

Figure 3. 3 Comparison between combined theoretical prediction of Eq (3.1) and Eq (3.2) and combined Coffin-Manson-Basquin curve for Al 7075-T6.

For Al 2024-T3, the predicted fatigue life by Eq $(3.1)\left(R_{s} \approx 1 / 3\right)$ and the Coffin-Manson relation are compared in Figure 3.4. It is observed that the agreement between $\mathrm{Eq}$ (3.1) and the Coffin-Manson (experimental) relation is reasonably good in the LCF region (plastic strain above 0.001$)$. The reason why Eq (3.1) overestimates the fatigue life could be attributed to the existence of crashed inclusions in the Al 2024-T3 plate during manufacturing [90]. Again, for the same reason as discussed for Al 7075-T6, the CoffinManson relation and Eq (3.1) depart further away at plastic strain $<0.001$. 


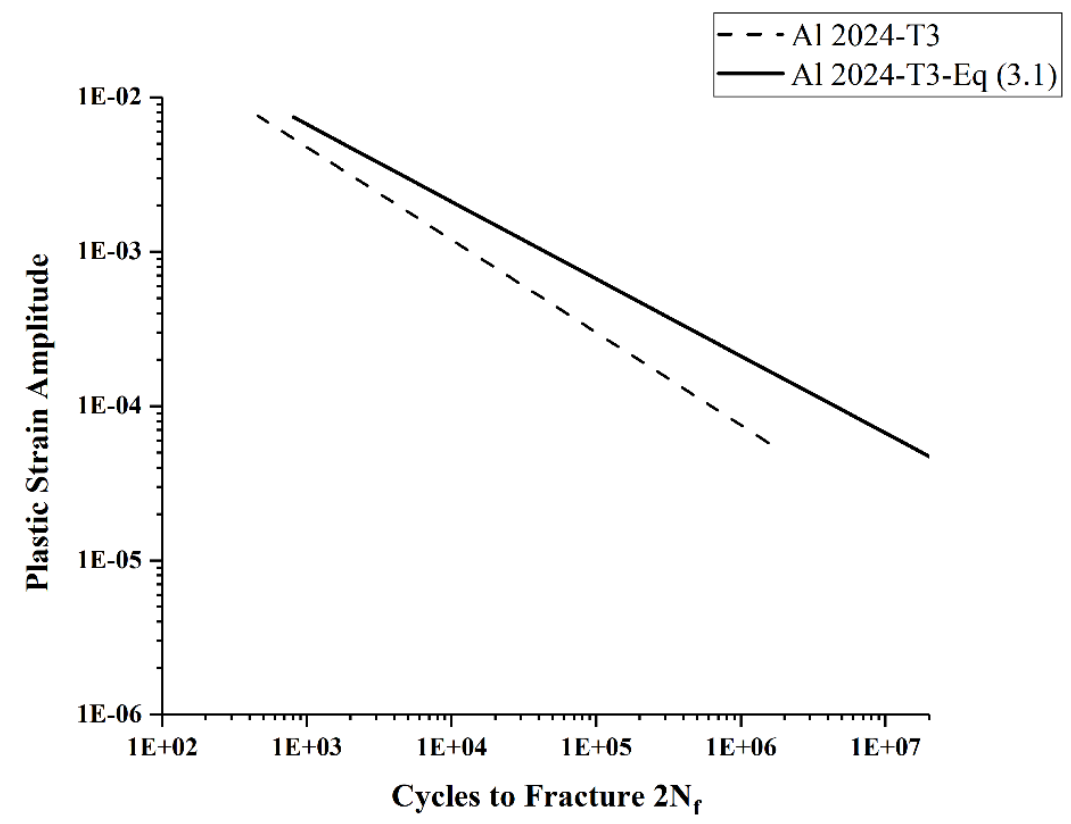

Figure 3. 4 Comparison between theoretical prediction of Eq (3.1) and Coffin-Manson curve for Al 2024-T3.

Similar to the case of Al 7075-T6, the predictions of Eq (3.2) achieve a good agreement with Basquin curve in the HCF region $\left(N_{f}>10^{4}\right.$ cycles) for Al 2024-T3, as shown in Figure 3.5. Once again, Eq (3.1) for the LCF region and Eq (3.2) for the HCF region are plotted together in a total strain-life plot in Figure 3.6, in comparison with the combined CoffinManson-Basquin curve. The prediction appears to well coincide with the experimental fitting. Particularly, Eq (3.1) is effective for the LCF region (plastic strain above 0.001), while for the HCF region where deformation is macroscopically elastic below the yield point, Eq (3.2) is effective once $\sigma_{0}$ is calibrated with one fatigue test. The TMW model has shown to be capable of predicting fatigue life for Al 2024-T3. 


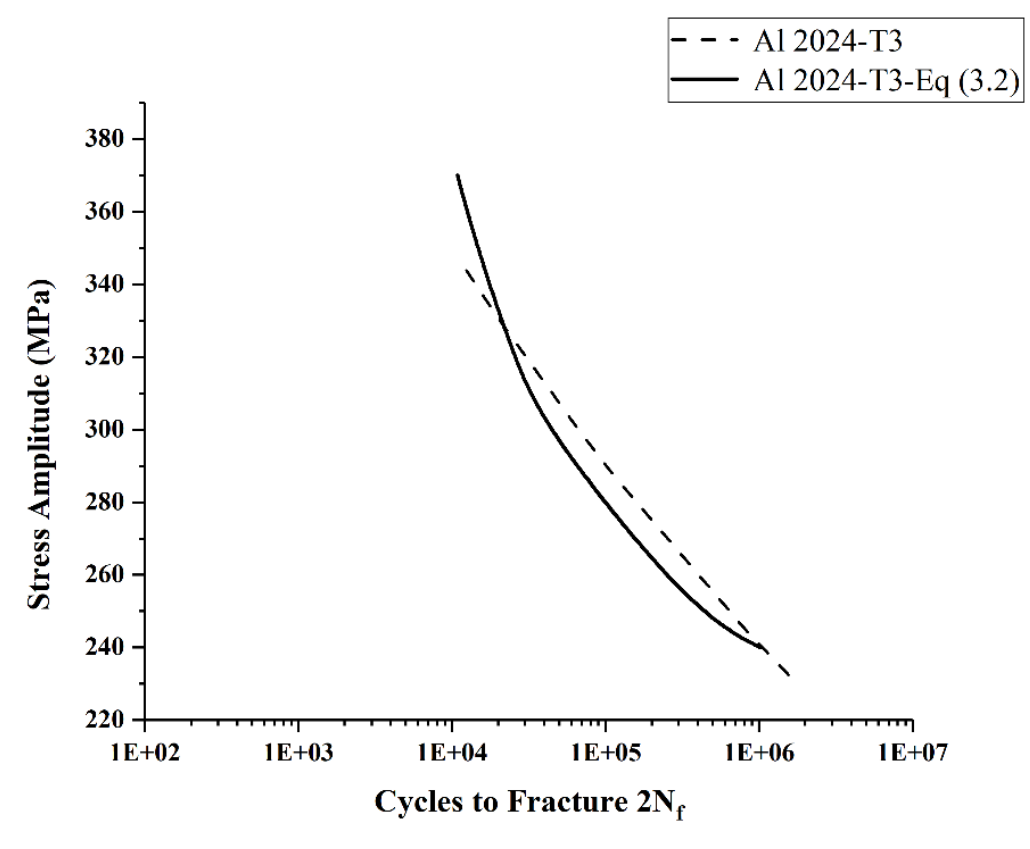

Figure 3. 5 Comparison between theoretical prediction of Eq (3.2) and Basquin curve for Al 2024-T3.

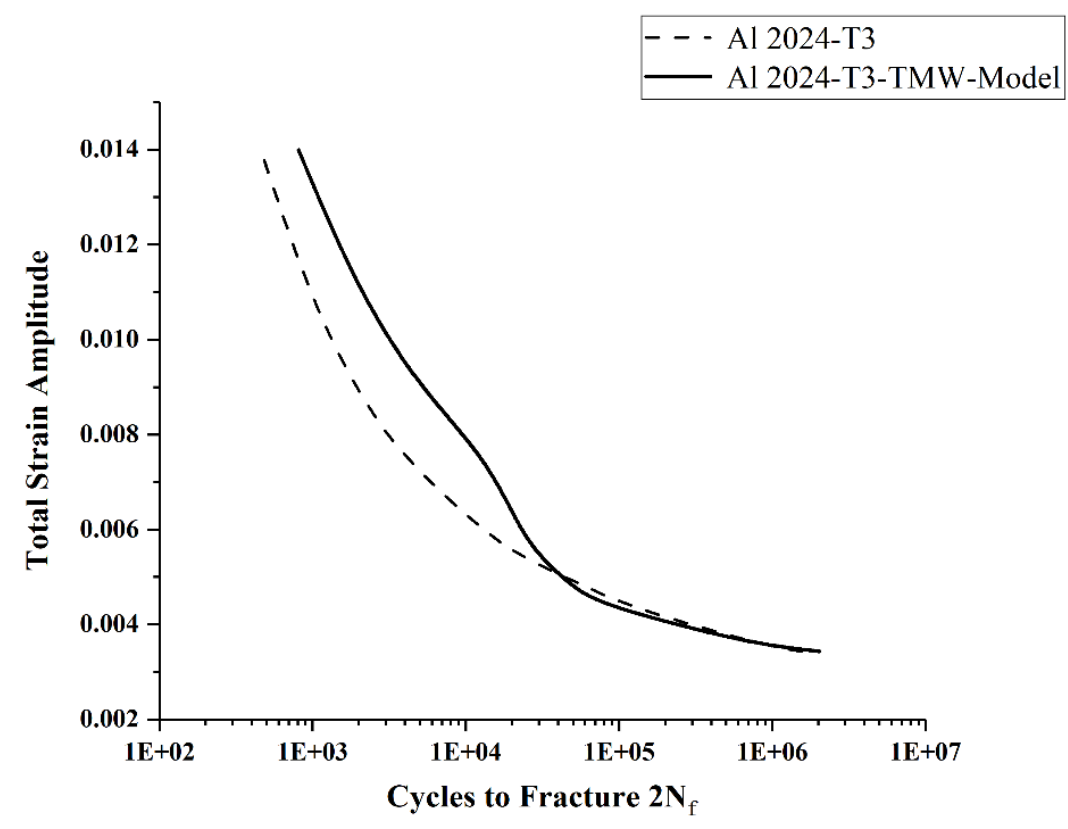

Figure 3. 6 Comparison between combined theoretical prediction of equation (3.1) and (3.2) and combined Coffin-Manson-Basquin curve for Al 2024-T3. 
Similar results are found for the case of SAE 1020, Eq (3.1) is effective for the LCF region (plastic strain amplitude above 0.001) and Eq (3.2) for the HCF region where deformation is macroscopically elastic below the yield point, once $\sigma_{0}$ is calibrated to the fatigue life of around $10^{7}$ cycles, as shown in Figure 3.7 and 3.8, respectively.

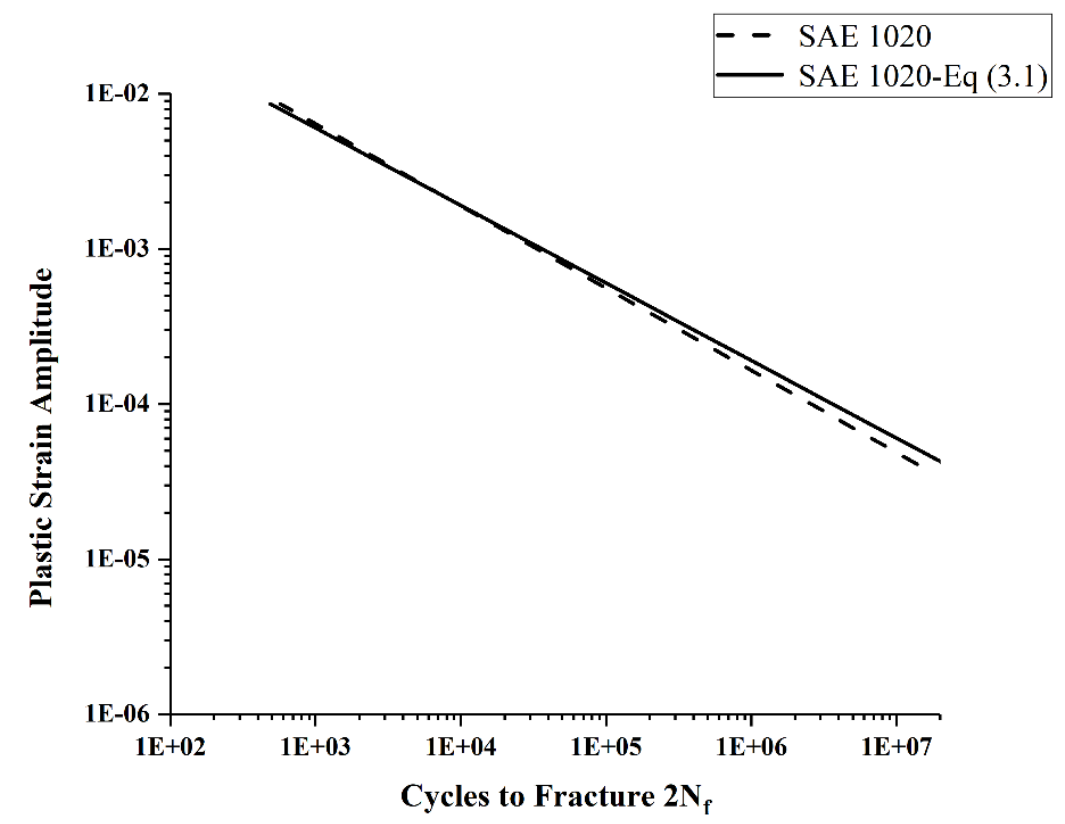

Figure 3. 7 Comparison between theoretical prediction of Eq (3.1) and Coffin-Manson curve for SAE 1020. 


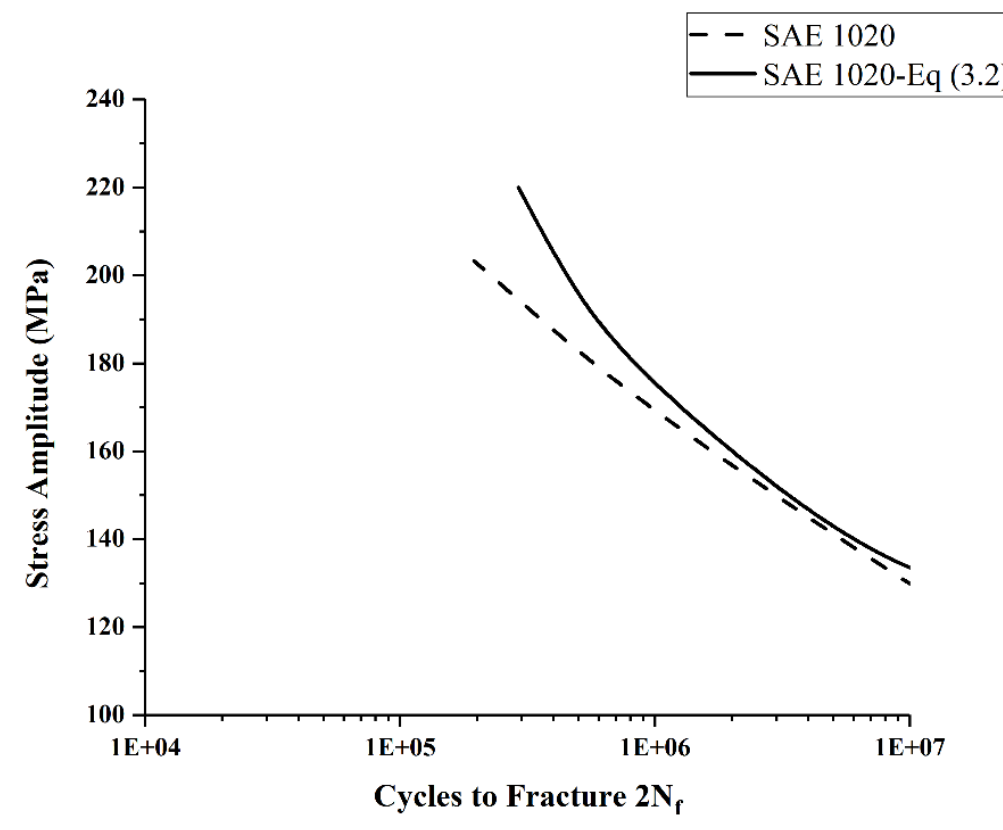

Figure 3. 8 Comparison between theoretical prediction of Eq (3.2) and Basquin curve for SAE 1020 .

The predictions of Eq (3.1) for the LCF region (plastic strain amplitude greater than 0.001) and predictions of $\mathrm{Eq}$ (3.2) for the HCF region are shown together in a total strain-life plot for SAE 1020 in Figure 3.9, in comparison with the combined Coffin-Manson-Basquin curve. The TMW model has shown to be valid for the fatigue life prediction of SAE 1020 . 


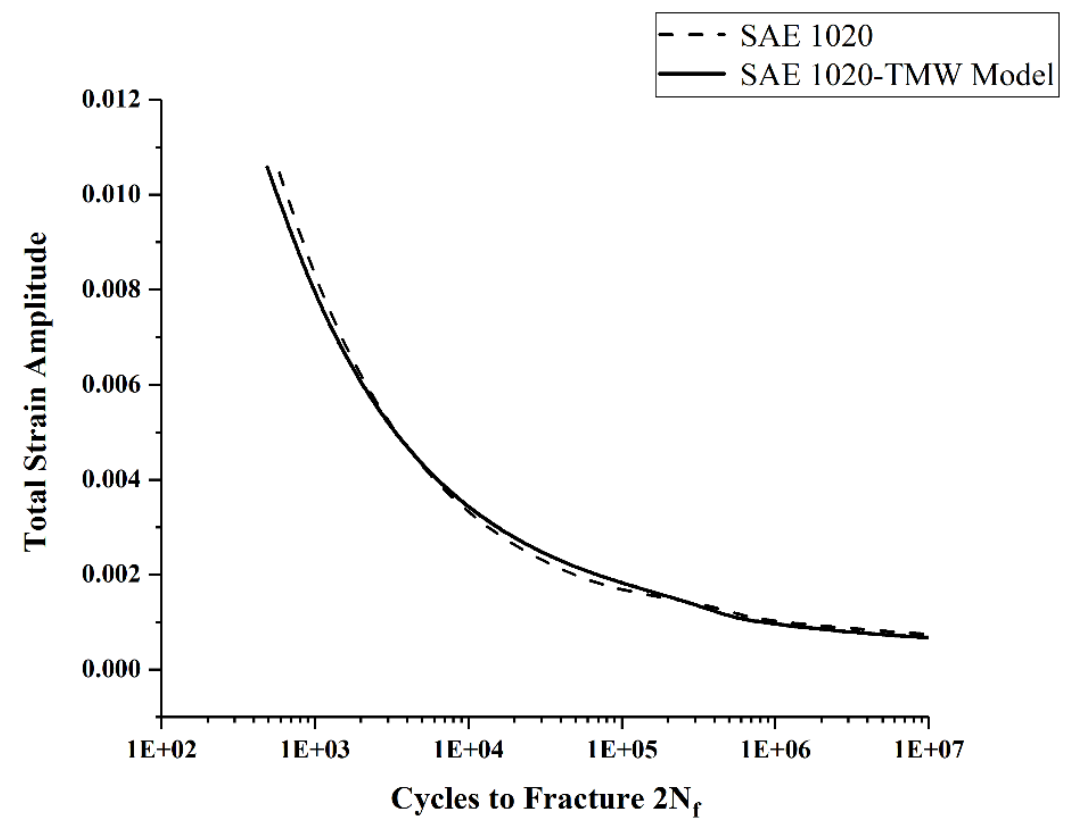

Figure 3. 9 Comparison between combined theoretical prediction of Eq (3.1) and Eq (3.2) and combined Coffin-Manson-Basquin curve for SAE 1020.

The predicted fatigue life curves by $\mathrm{Eq}(3.1)\left(R_{S} \approx 1 / 3\right)$ and the Coffin-Manson relation for SAE 4340 are shown in Figure 3.10 for comparison. Similar to the previous cases, Eq (3.1) provides accurate predictions of fatigue life in the LCF region at plastic strain amplitude above 0.001 . The stress-life relations by $\mathrm{Eq}$ (3.2) and Basquin equation, Eq (3.4), are shown in Figure 3.11 for the HCF region below the yield strength $\sigma_{y s}$. The predictions of Eq (3.2) well coincide with the Basquin curve in the HCF region $\left(N_{f}>10^{4}\right.$ cycles) with a scatter factor of 2 . 


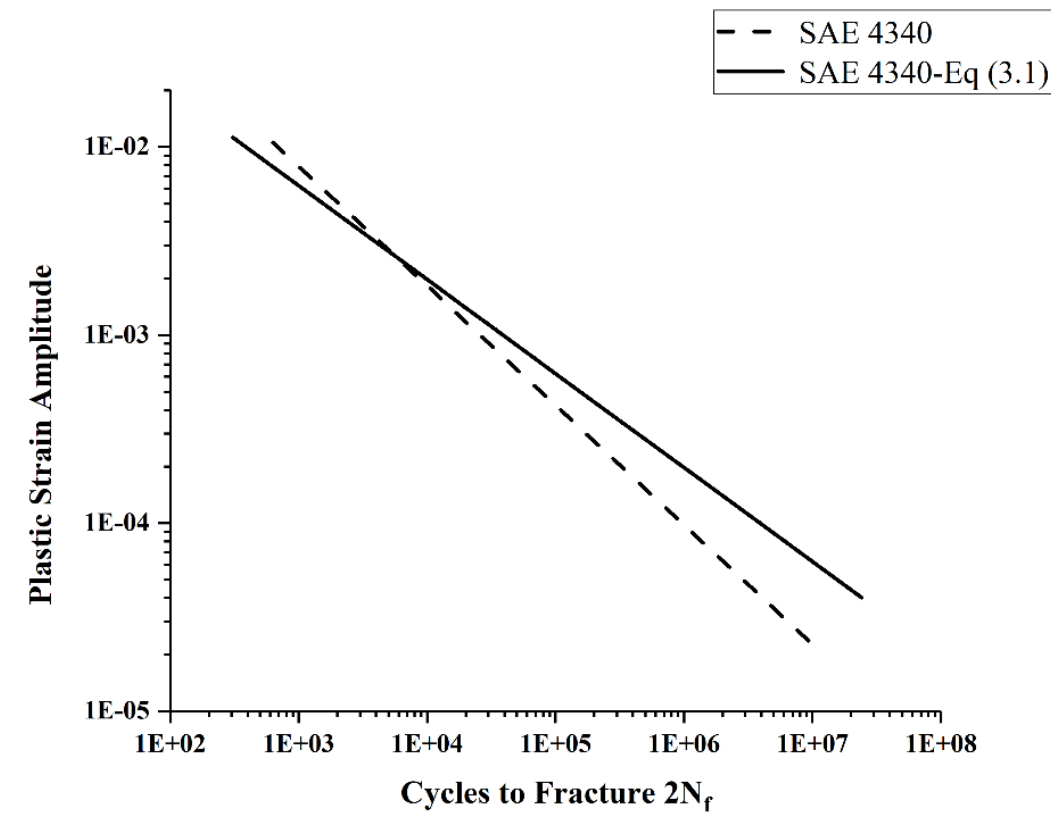

Figure 3. 10 Comparison between theoretical prediction of Eq (3.1) and Coffin-Manson curve for SAE 4340.

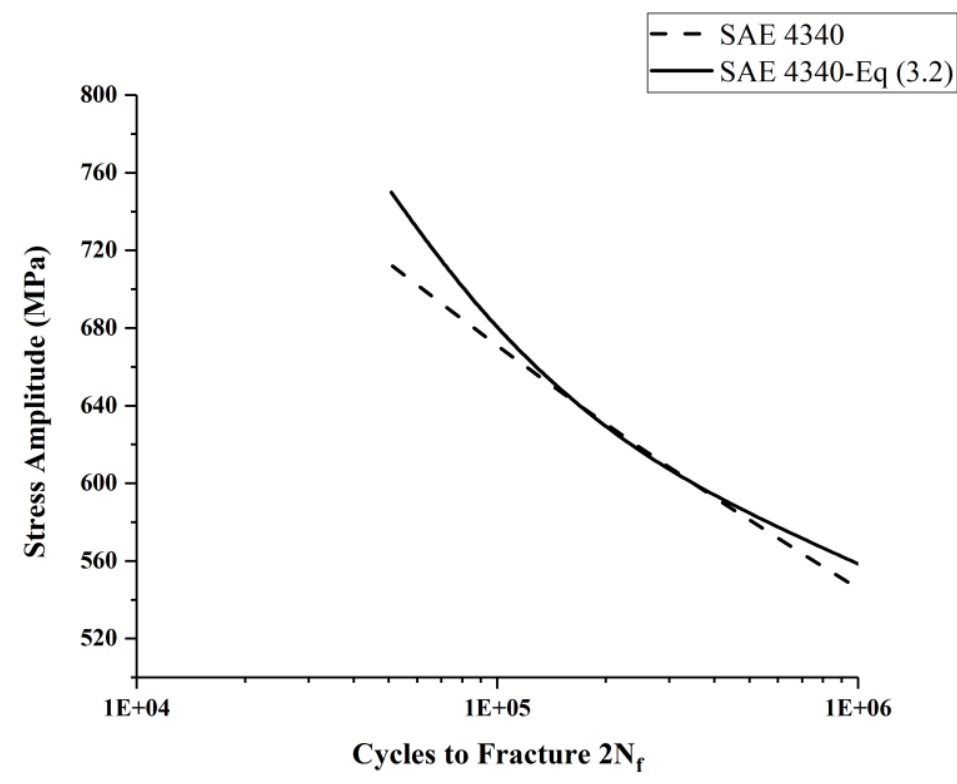

Figure 3. 11 Comparison between theoretical prediction of $\mathrm{Eq}$ (3.2) and Basquin curve for SAE 4340 .

The predictions of Eq (3.1) for the LCF region (plastic strain greater than 0.001) and the 
predictions of Eq (3.2) for the HCF region are shown together in a total strain-life plot for SAE 4340 in Figure 3.12, in comparison with the combined Coffin-Manson-Basquin curve. The overall validity of the TMW model for SAE 4340 has been proven as the prediction is very close to the experimental curve, given the assumed roughness condition. Particularly, Eq (3.1) is effective for the LCF region (plastic strain amplitude above 0.001), while for the HCF region where deformation is macroscopically elastic below the yield point, Eq (3.2) is effective once $\sigma_{0}$ is calibrated with the one fatigue test. The TMW model has shown to be capable of predicting fatigue life for SAE 4340 .

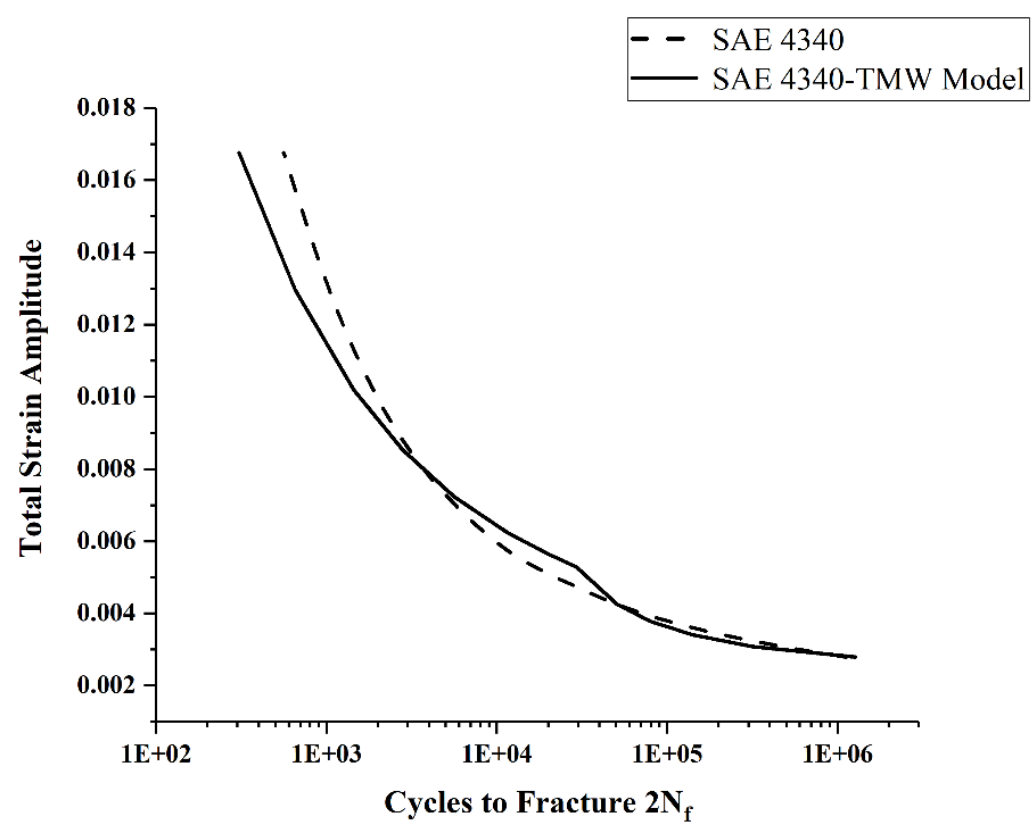

Figure 3. 12 Comparison between combined theoretical prediction of $\mathrm{Eq}$ (3.1) and $\mathrm{Eq}$ (3.2) and combined Coffin-Manson-Basquin curve for SAE 4340.

The predicted fatigue life for Ti-6Al-4V by Eq $(3.1)\left(R_{s} \approx 1 / 3\right)$ and the Coffin-Manson relation are shown in Figure 3.13 for comparison. Again, fairly good agreement is found 
between Eq (3.1) and Coffin-Manson curve in the LCF region (plastic strain amplitude above 0.001 ). As to the stress-based $\mathrm{Eq}(3.2)$, the stress-life relations by $\mathrm{Eq}(3.2)$ and Basquin equation, Eq (3.4), are shown in Figure 3.14 for the HCF region below the yield strength $\sigma_{y s}$. In the HCF region $\left(N_{f}>10^{4}\right.$ cycles), the predictions of Eq (3.2) well agree with the Basquin curve.

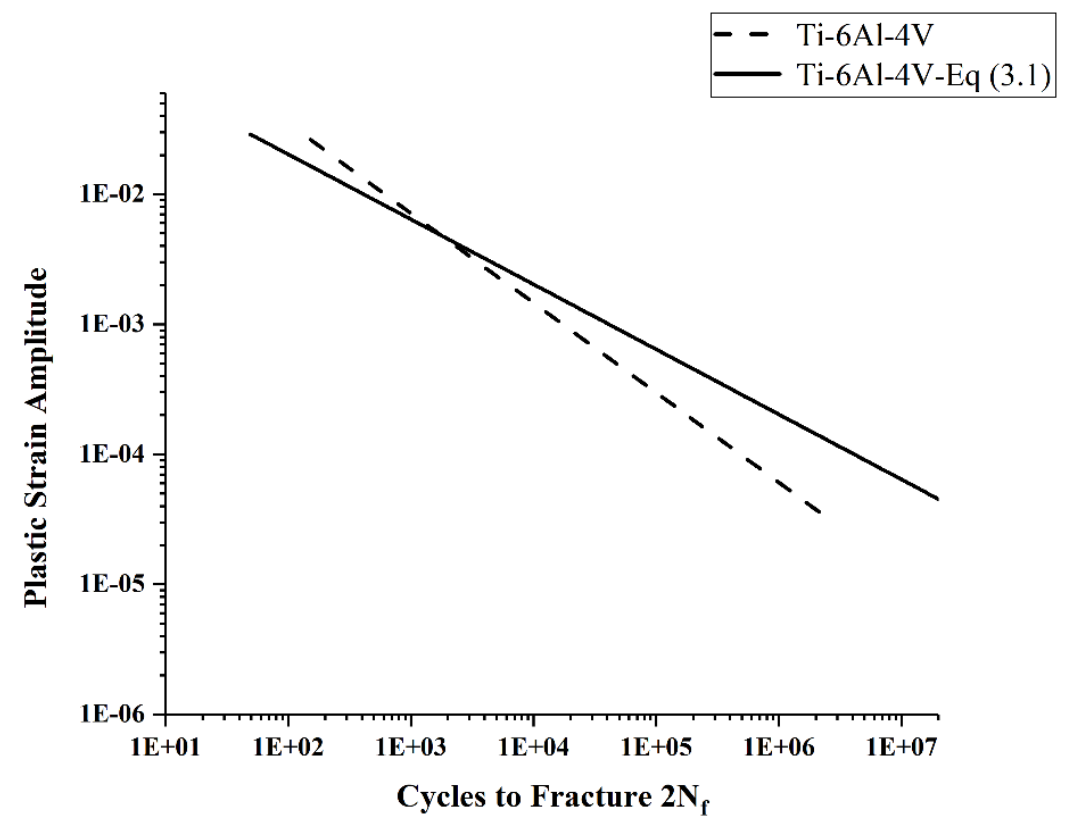

Figure 3. 13 Comparison between theoretical prediction of Eq (3.1) and Coffin-Manson curve for Ti-6Al-4V. 


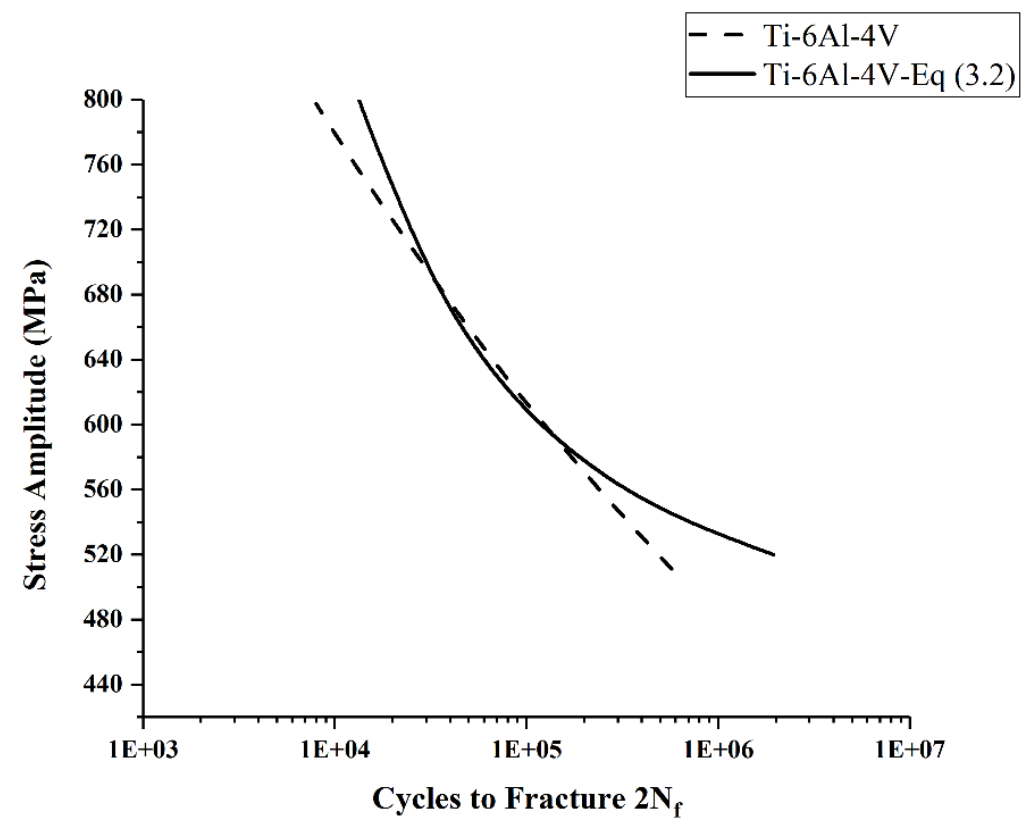

Figure 3. 14 Comparison between theoretical prediction of $\mathrm{Eq} \mathrm{(3.2)} \mathrm{and} \mathrm{Basquin} \mathrm{curve} \mathrm{for}$ Ti-6Al-4V.

The predictions of Eq (3.1) for the LCF region (plastic strain greater than 0.001) and the predictions of $\mathrm{Eq}$ (3.2) for the HCF region are shown together in a total strain-life plot for Ti-6Al-4V in Figure 3.15, in comparison with the combined Coffin-Manson-Basquin curve. The overall validity of the TMW model for Ti-6Al-4V can be proven as the predictions are generally consistent to the experimental curve, given the assumed roughness condition. The Eq (3.1) predictions are effective for the LCF region (plastic strain above 0.001), while for the HCF region where deformation is macroscopically elastic below the yield point, Eq. (3.2) is effective once $\sigma_{0}$ is calibrated with one fatigue test. The TMW model has demonstrated to be capable of predicting fatigue life for Ti-6Al-4V. 


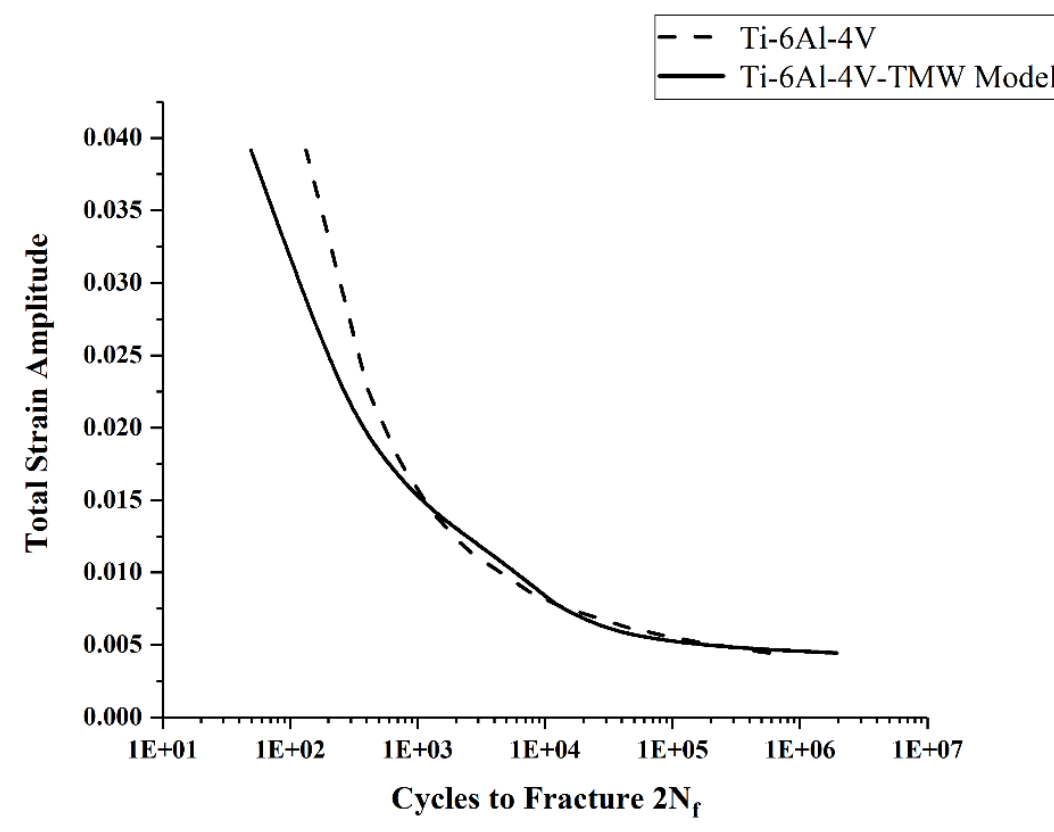

Figure 3. 15 Comparison between combined theoretical prediction of Eq (3.1) and Eq (3.2) and combined Coffin-Manson-Basquin curve for Ti-6Al-4V.

The predicted fatigue life for Inconel 617 by Eq $(3.1)\left(R_{S} \approx 1 / 3\right)$ and the Coffin-Manson relation are shown in Figure 3.16 for comparison, the agreement between Eq (3.1) and Coffin-Manson curve in the LCF region (plastic strain amplitude above 0.001) is reasonably good. As to the stress-based Eq (3.2), the stress-life relations by Eq (3.2) and Basquin equation, $\mathrm{Eq}$ (3.4) are illustrated in Figure 3.17 for the HCF region below the yield strength $\sigma_{y s}$. In this region ( $N_{f}>10^{5}$ cycles), the predictions of Eq (3.2) well agree with the Basquin curve. 


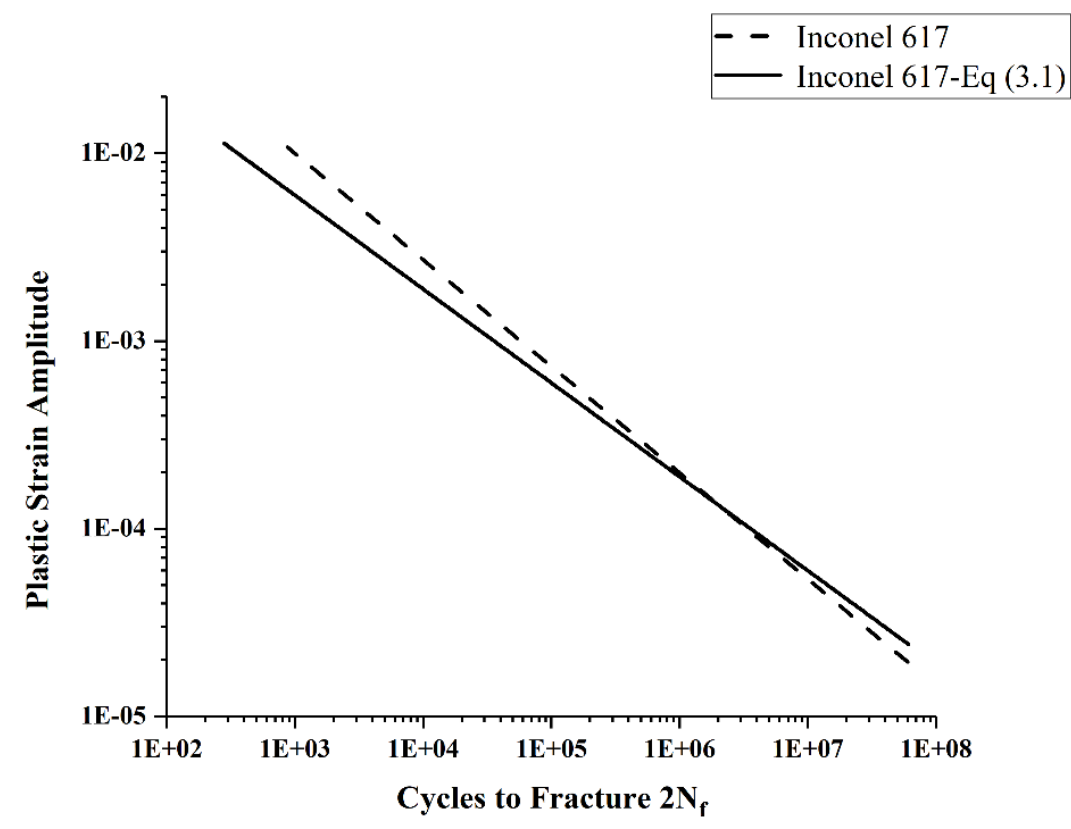

Figure 3. 16 Comparison between theoretical prediction of Eq (3.1) and Coffin-Manson curve for Inconel 617.

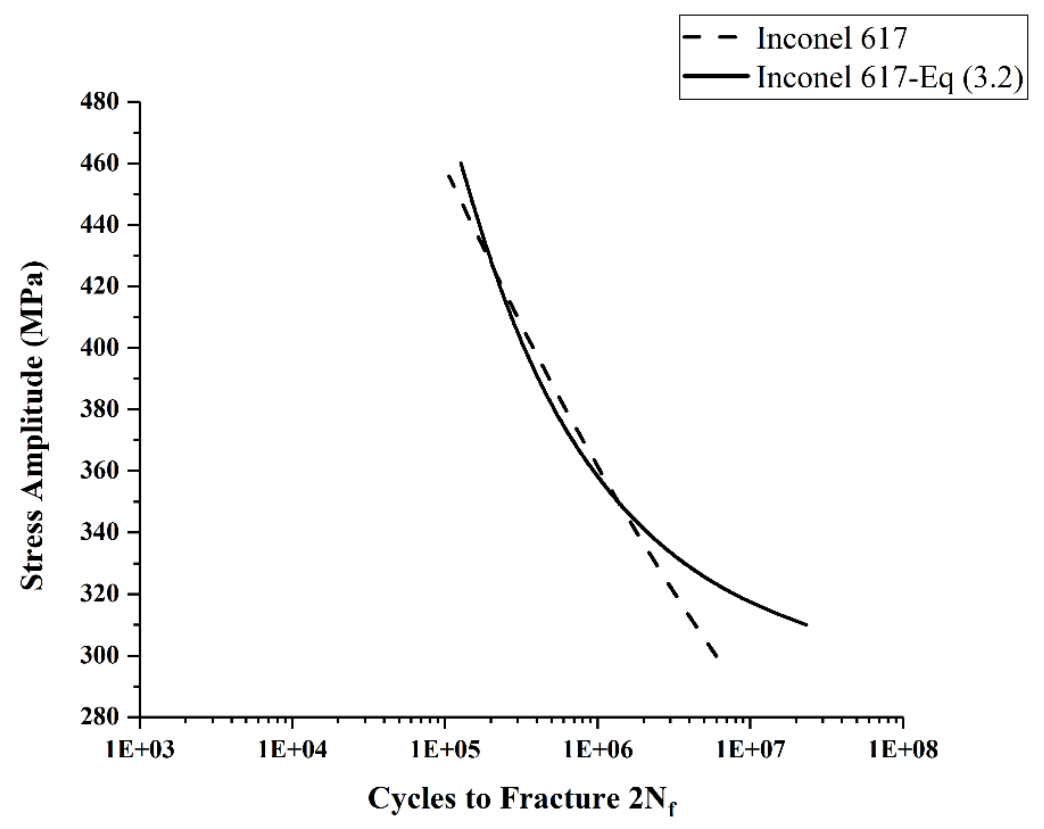

Figure 3. 17 Comparison between theoretical prediction of Eq (3.2) and Basquin curve for Inconel 617. 
Similar to previous cases, the predictions of Eq (3.1) for the LCF region (plastic strain amplitude greater than 0.001) and the predictions of Eq (3.2) for the HCF region for Inconel 617 are shown together in a total strain-life plot in Figure 3.18, in comparison with the combined Coffin-Manson-Basquin curve. The TMW model has shown to be capable of predicting fatigue life for Inconel 617.

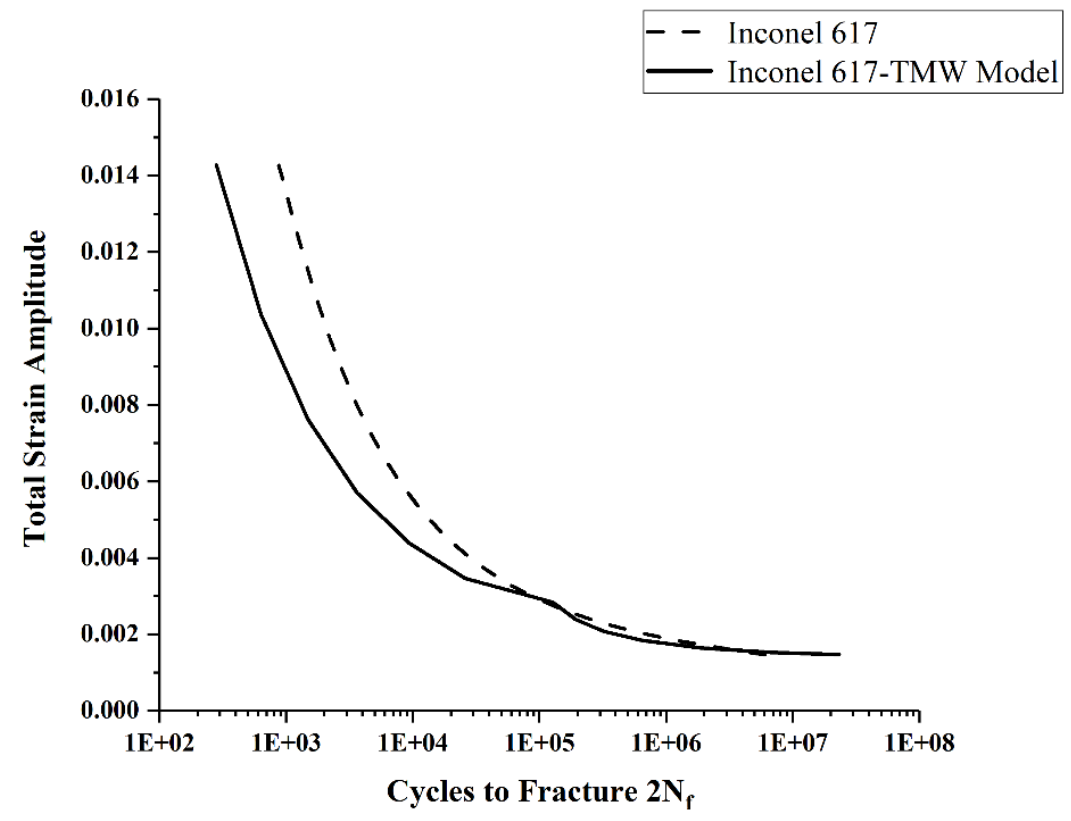

Figure 3. 18 Comparison between combined theoretical prediction of $\mathrm{Eq}(3.1)$ and $\mathrm{Eq}$ (3.2) and combined Coffin-Manson-Basquin curve for Inconel 617.

The predicted fatigue life of Inconel 718 by Eq $(3.1)\left(R_{S} \approx 1 / 3\right)$ and the Coffin-Manson curve are shown in Figure 3.19 for comparison. Good agreement is observed between Eq (3.1) and the experimental Coffin-Manson curve in the LCF region (at plastic strain amplitude above 0.001), despite larger deviations at the plastic strain amplitude below 
0.001. As to the stress-based Eq (3.2), the stress-life relations by Eq. (3.2) and Basquin equation, $\mathrm{Eq}$ (3.4), are illustrated in Figure 3.20 for the HCF region below the yield strength $\sigma_{y s}$. In this region $\left(N_{f}>10^{4}\right.$ cycles), the predictions of $\mathrm{Eq}$ (3.2) are very close to the Basquin curve.

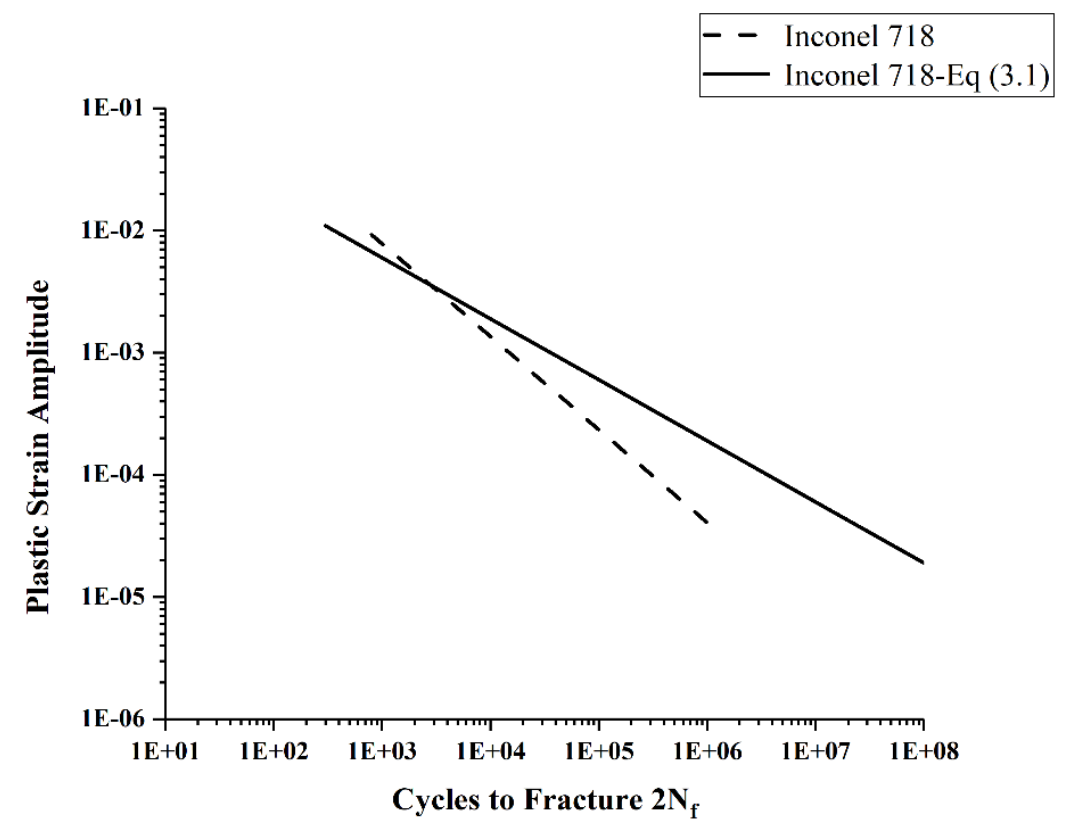

Figure 3. 19 Comparison between theoretical prediction of Eq (3.1) and Coffin-Manson curve for Inconel 718. 


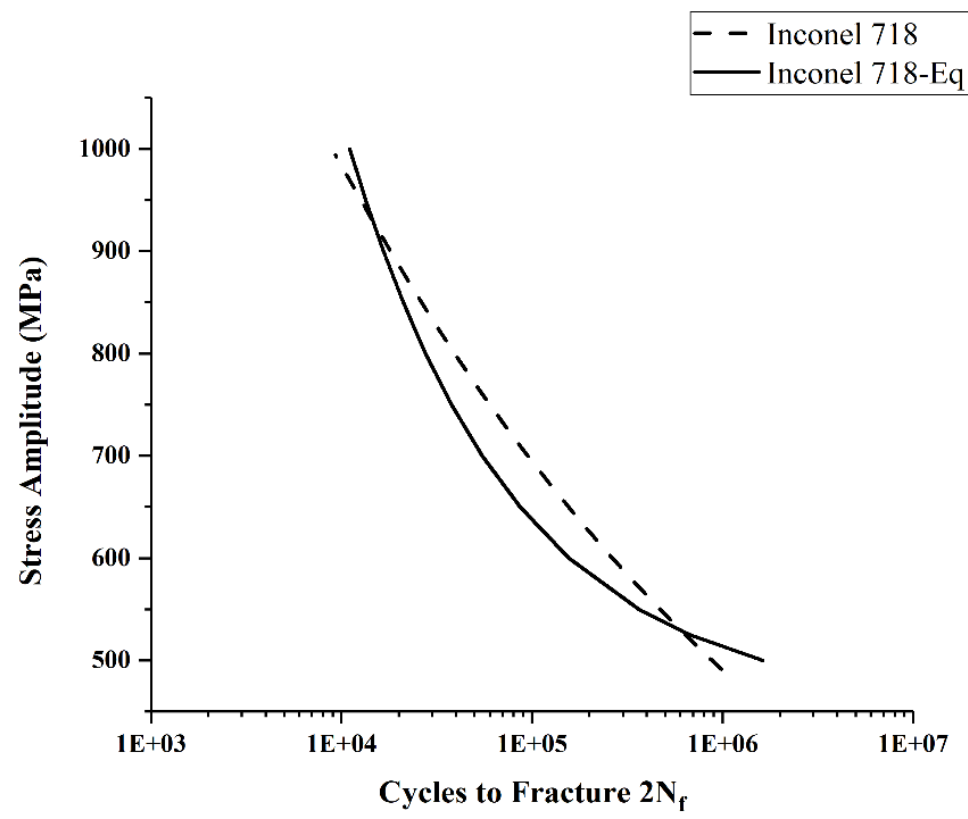

Figure 3. 20 Comparison between theoretical prediction of Eq (3.2) and Basquin curve for Inconel 718.

Finally, the predictions of Eq (3.1) for the LCF region (plastic strain greater than 0.001) and the predictions of Eq (3.2) for the HCF region are shown together in a total strain-life plot for Inconel 718 in Figure 3.21, in comparison with the combined Coffin-MansonBasquin curve. The TMW model has demonstrated to be capable of predicting the fatigue life of Inconel 718. 


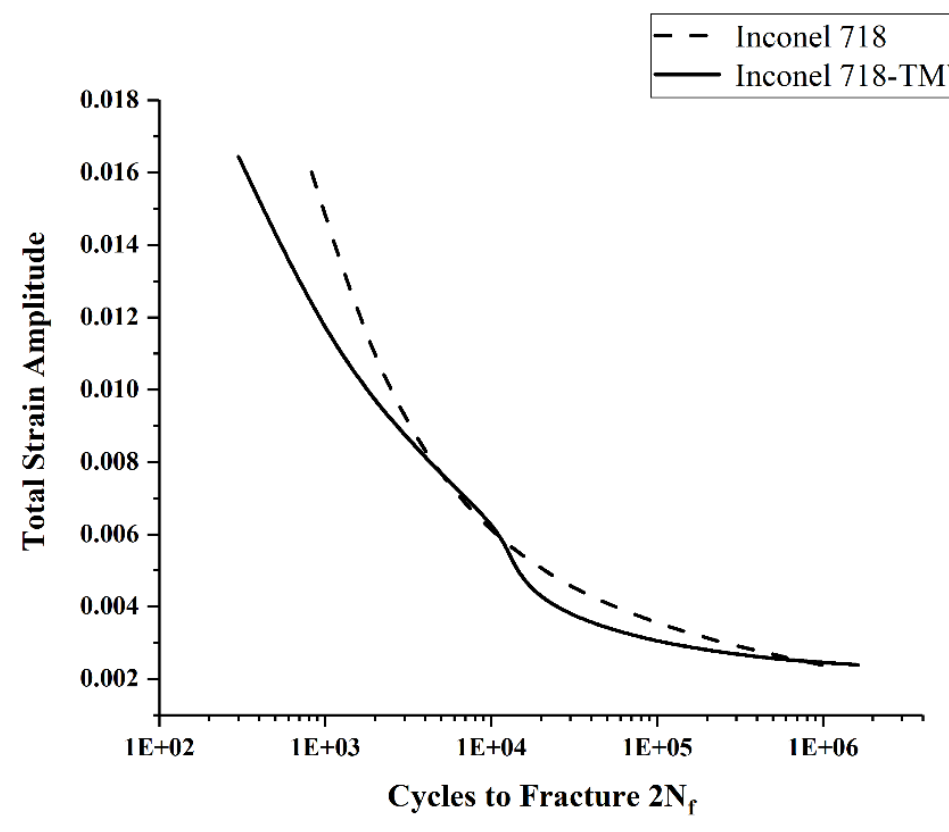

Figure 3. 21 Comparison between combined theoretical prediction of Eq (3.1) and Eq (3.2) and combined Coffin-Manson-Basquin curve for Inconel 718.

In terms of Haynes 282, the predictions of Eq (3.1) is compared with the experimental data from literature [84] in Figure 3.22. Similar to the previous cases, the predictions of Eq (3.1) agree very well experimental data in the LCF region (plastic strain smaller than 0.001 ). In Figure 3.23, the predictions of Eq (3.2) are found to perform well when applied stress is below the yielding strength of Haynes 282 as compared to the experimental data. Figure 3.24 shows the comparison between the total strain-life plot consisting of predictions of Eq (3.1) for the LCF region (plastic strain greater than 0.001) and the predictions of Eq (3.2) for the HCF region, and experimental data, which validates the TMW model for estimating the fatigue life of Haynes 282. 


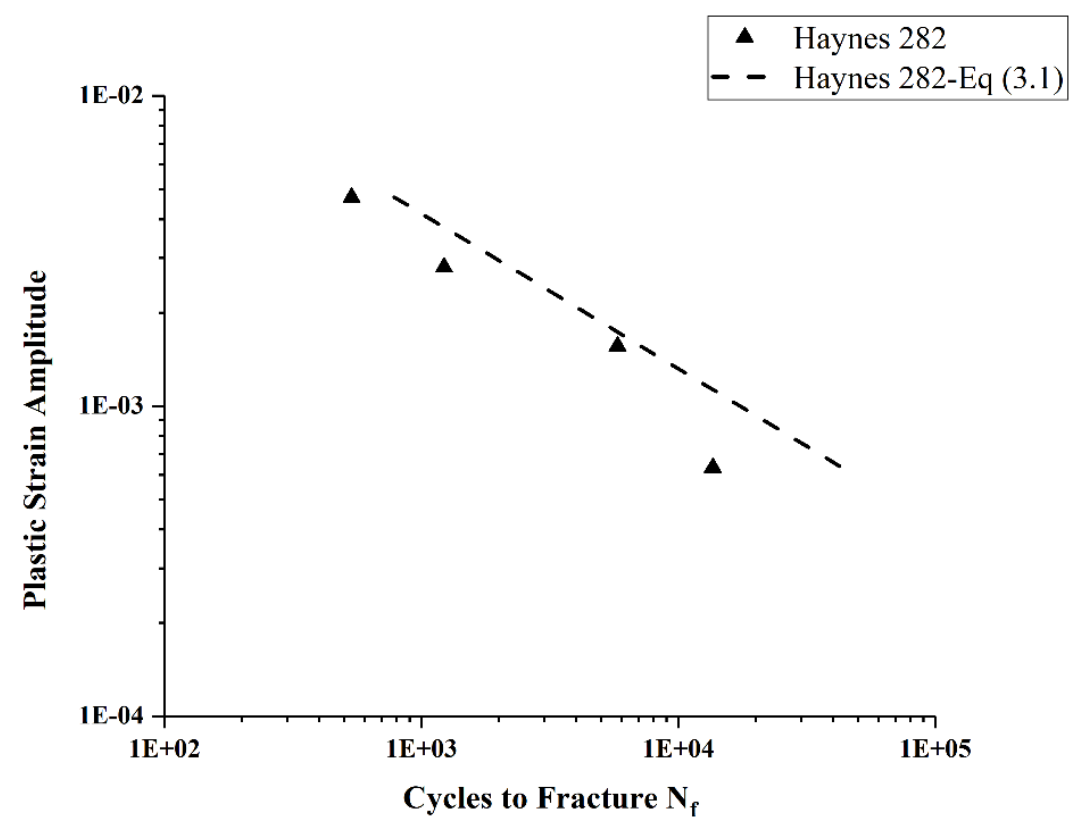

Figure 3. 22 Comparison between theoretical prediction of Eq (3.1) and Coffin-Manson curve for Haynes 282.

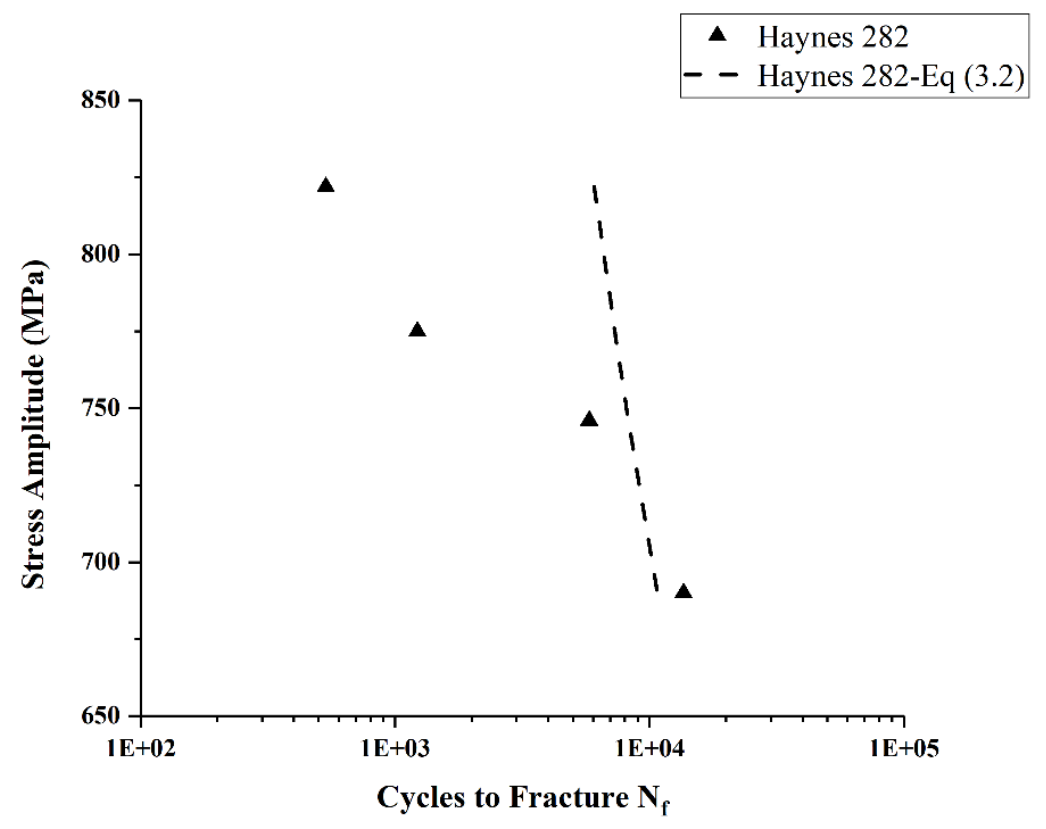

Figure 3. 23 Comparison between theoretical prediction of Eq (3.2) and Basquin curve for Inconel Haynes 282. 


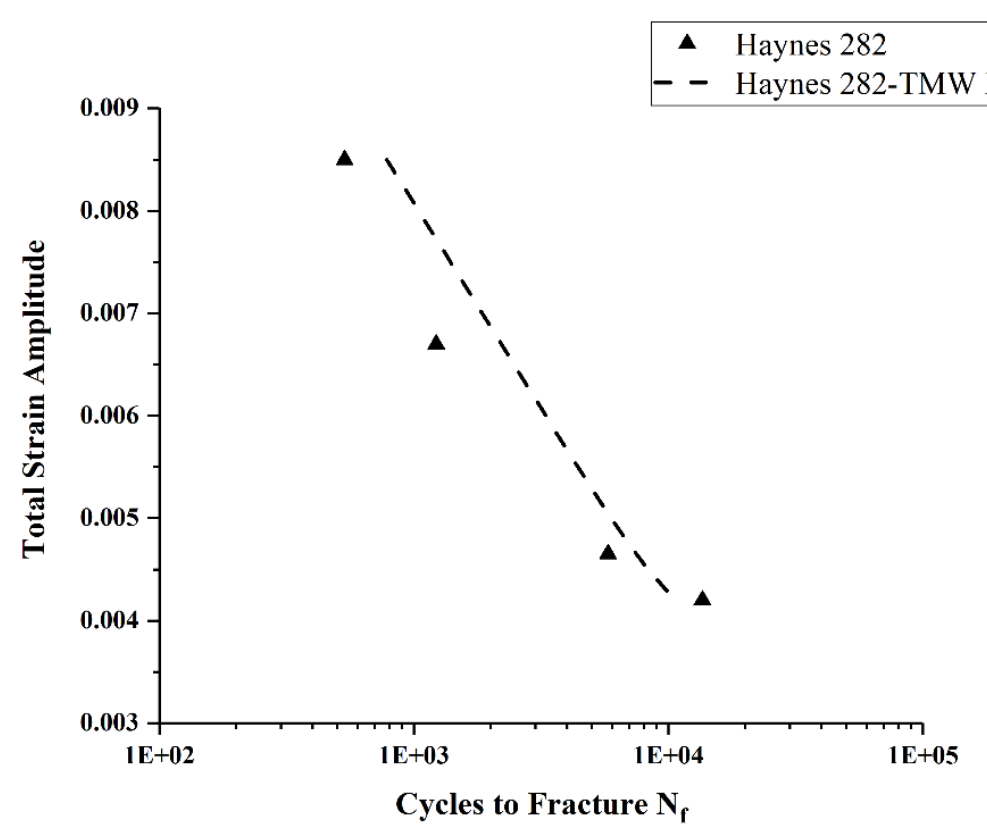

Figure 3. 24 Comparison between combined theoretical prediction of Eq (3.1) and Eq (3.2) and combined Coffin-Manson-Basquin curve for Haynes 282.

\subsection{Surface Roughness Factor in the TMW Model}

\subsubsection{Surface roughness parameters}

Surface roughness, as one of the important characteristics for surface topography, has significant effect on the fatigue crack nucleation life of structrual materials in engineering. The reason is that cracks are prone to appear at free surfaces of cyclically loaded materials where plastic strain is always concentrated. In the TMW model, roughness effect has been taken into account by introducing a roughess factor $R_{S}$ to $\mathrm{Eq}$ (3.1) and Eq (3.2). In the previous section, $R_{S}$ has been assumed to be $1 / 3$ and 1 in $\mathrm{Eq}(3.1)$ and $\mathrm{Eq}(3.2)$ respectively, for the fatigue life predictions of the selected eight materials. In addition, the microstructure-dependent parameter $\sigma_{0}$ in $\mathrm{Eq}$ (3.2) is also affected by suface roughness. 
However, in engineering surface roughness is always chracterized by various parameters such as arithmetical mean deviation of the assessed profile $R_{a}$, root mean squared $R_{q}$ and the maximum peak height $R_{p}$ through experimental measurement. Therefore, a methematical relationship between profile roughness parameters and roughnes factor $R_{S}$ needs to be derived so that the effect of surface roughness in predicted fatigue life by either $\mathrm{Eq}$ (3.1) or Eq (3.2) can be quantified. In this section, the relationship between the surface roughness factor $R_{s}$ and the profile roughness parameter $R_{a}$ is developed for an aluminum alloy and a medium-carbon steel based on the available fatigue data in literature [19] [20]. It illustrates an engineering approach to determining $R_{S}$ for fatigue design of structures with different surface finish.

\subsubsection{Influence of surface roughness on fatigue life}

The mechanical properties of the aluminum alloy and medium-carbon steel that are used in the previous research reported in literature are given in Table 3.5. Figure 3.25 and Figure 3.26 show the experimental fatigue lives with different values of $R_{a}$ plotted against the applied stress amplitude for aluminum alloy and medium-carbon steel. It can be clearly seen that for both materials, at all stress amplitudes the larger the arithmetical mean deviation of the assessed profile $R_{a}$ value, the shorter the fatigue life is. Additionally, it seems that in the LCF region where the stress amplitude is well above the yield strength of the material the logarithmic fatigue life for different $R_{a}$ appears much more dispersed than that in the HCF region for both materials. This indicates that surface roughness effect is 
more pronounced in the LCF region than in the HCF region for the aluminum alloy and medium-carbon steel. Consequently, the experimental data are fitted by Eq (3.2) separately for the LCF and HCF regions to analyze the correlation between $R_{s}$ and $R_{a}$.

Table 3. 5 Monotonic mechanical properties of materials for surface roughness factor study [19] [20]

\begin{tabular}{|c|c|c|c|c|}
\hline Material & $\mathrm{E}(\mathrm{GPa})$ & $\sigma_{y s}(\mathrm{MPa})$ & $\sigma_{\mathrm{u}}(\mathrm{MPa})$ & $\mathrm{EI}(\%)$ \\
\hline Aluminum alloy & 71 & 277 & 315 & 11 \\
\hline Medium-carbon steel & 200 & 490 & 710 & 19.17 \\
\hline
\end{tabular}

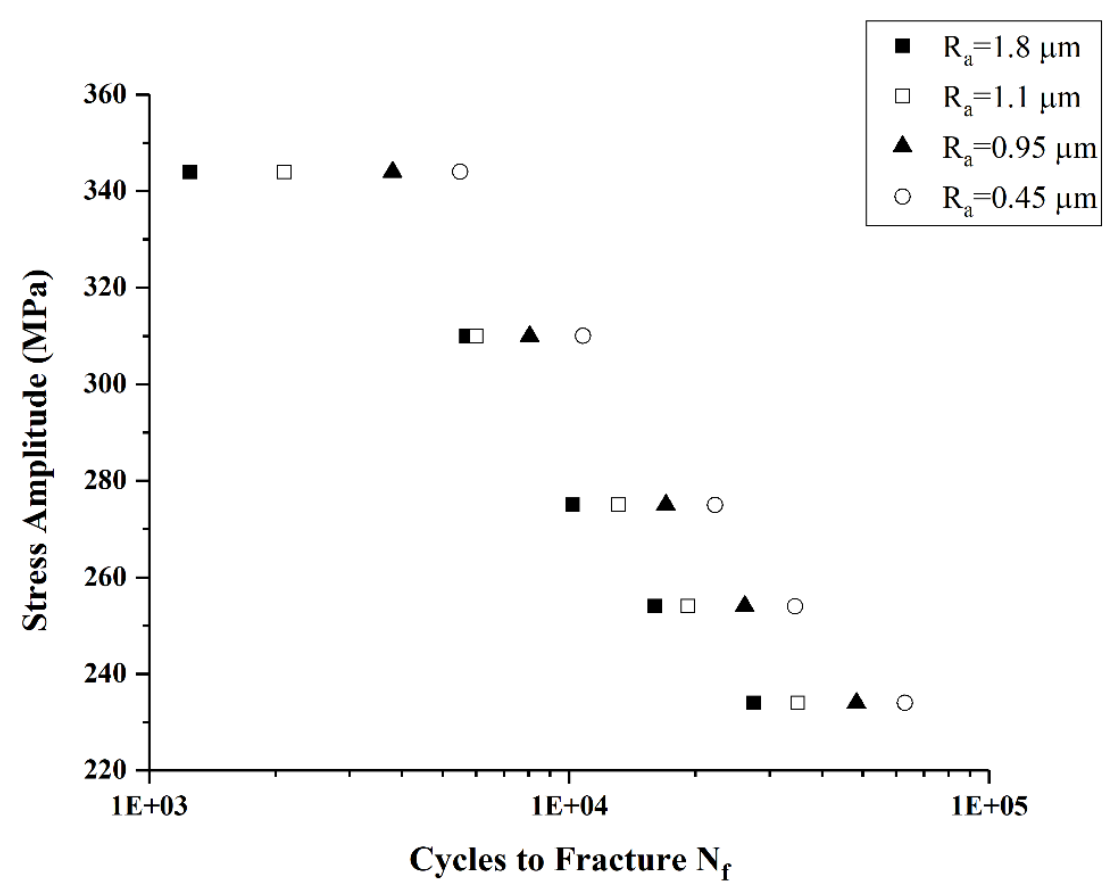

Figure 3. 25 Experimental fatigue life of aluminum alloy with different arithmetical mean deviation of the assessed profile $R_{a}$ values against stress amplitude [103]. 


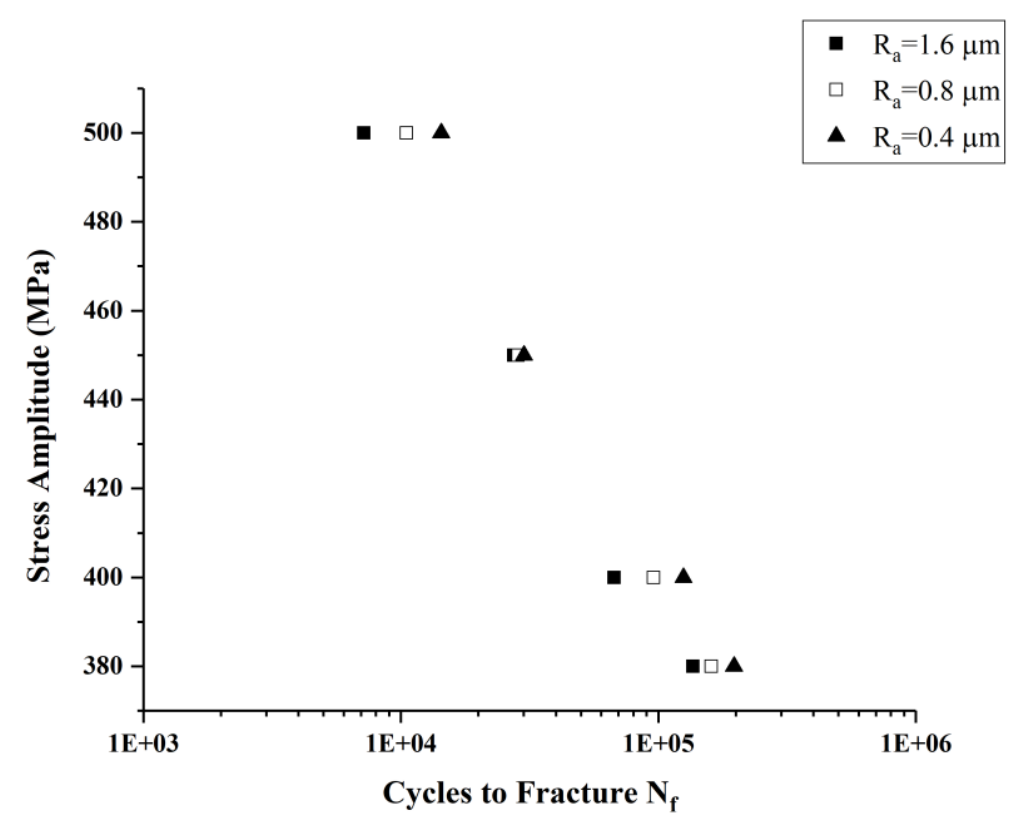

Figure 3. 26 Experimental fatigue life of medium-carbon steel with different arithmetical mean deviation of the assessed profile $R_{a}$ values against stress amplitude [102].

\subsubsection{Surface roughness effects on $\mathrm{HCF}$}

For the HCF cases of aluminum alloy (stress amplitudes of $234 \mathrm{MPa}, 254 \mathrm{MPa}, 275 \mathrm{MPa}$ and $310 \mathrm{MPa}$ ) and medium-carbon steel (stress amplitudes of $380 \mathrm{MPa}, 400 \mathrm{MPa}$ and 450 $\mathrm{MPa}$ ), analytical roughness factor $R_{S}$ and lattice resistance $\sigma_{0}$ with roughness parameter $R_{a}$ ranging from 0.45 to $1.80 \mu \mathrm{m}$ are obtained by fitting the experimental fatigue lives using Eq (3.2). As presented in Table 3.6, the related parameters for the materials are obtained similarly as described in subsection 3.2.2. 
Table 3. 6 Fatigue coefficients of the TMW model for the materials used in surface roughness analysis

\begin{tabular}{|c|c|c|c|c|}
\hline Material & $v$ & $\mu(\mathrm{GPa})$ & $\mathrm{w}_{\mathrm{s}}\left(\mathrm{J} / \mathrm{m}^{2}\right)$ & $\mathrm{b}_{\mathrm{v}}\left(10^{-10} \mathrm{~m}\right)$ \\
\hline Aluminum alloy & 0.32 & 26.89 & 1.121 & 2.86 \\
\hline Medium-carbon steel & 0.3 & 76.92 & 2.373 & 2.48 \\
\hline
\end{tabular}

The first step is to compute "analytical fatigue life" using the fatigue coefficients given in Table 3.6 and assumed analytical roughness factor $R_{S}$ and lattice resistance $\sigma_{0}$. Through adjustment of $R_{S}$ and $\sigma_{0}$, the analytical life is determined to be the best fitting for experimental data of two selected materials, as shown in Figure 3.27 and Figure 3.28. Then the analytical roughness factor $R_{s}$ and lattice resistance $\sigma_{0}$ with different values of $R_{a}$ are derived and presented in Table 3.7 and Table 3.8.

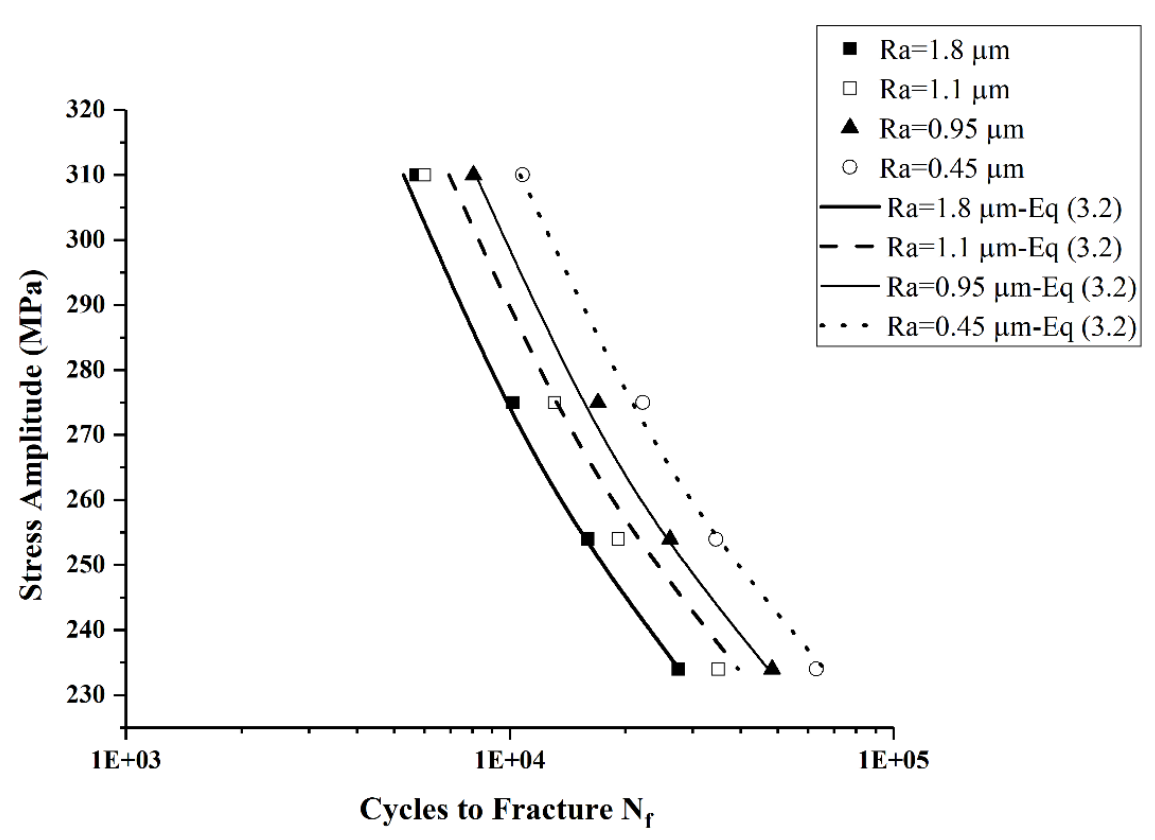

Figure 3. 27 Comparison of experimental fatigue life with analytical fatigue life by Eq (3.2) for aluminum alloy with different values of $R_{a}$. 


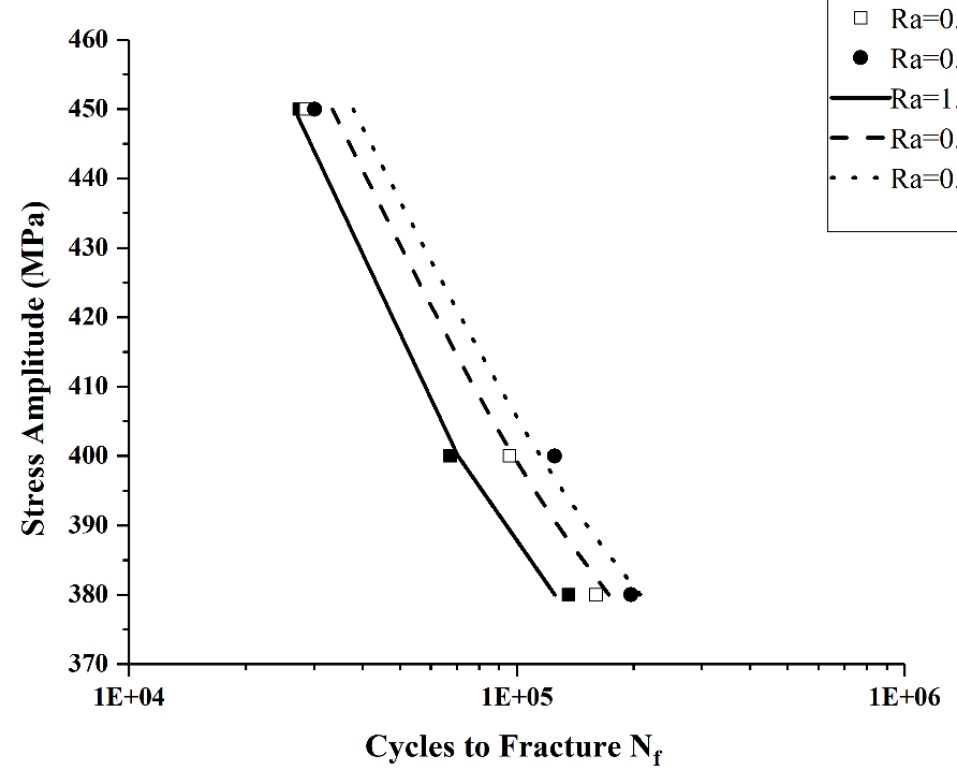

Figure 3. 28 Comparison of experimental fatigue life with analytical fatigue life by Eq (3.2) for medium-carbon steel with different values of $R_{a}$.

Table 3. 7 Analytical roughness factor $R_{S}$ and lattice resistance $\sigma_{0}$ for aluminum alloy

\begin{tabular}{|c|c|c|}
\hline $\mathrm{R}_{\mathrm{a}}(\mu \mathrm{m})$ & $\mathrm{R}_{\mathrm{s}}$ & $\sigma_{0}(\mathrm{MPa})$ \\
\hline 1.8 & 0.42 & 175 \\
\hline 1.1 & 0.52 & 179 \\
\hline 0.95 & 0.6 & 180 \\
\hline 0.45 & 0.75 & 183 \\
\hline
\end{tabular}

Table 3. 8 Analytical roughness factor $R_{S}$ and lattice resistance $\sigma_{0}$ for medium-carbon steel

\begin{tabular}{|c|c|c|}
\hline $\mathrm{R}_{\mathrm{a}}(\mu \mathrm{m})$ & $\mathrm{R}_{\mathrm{s}}$ & $\sigma_{0}(\mathrm{MPa})$ \\
\hline 1.8 & 0.74 & 320 \\
\hline 1.1 & 0.86 & 325 \\
\hline 0.95 & 0.92 & 328 \\
\hline
\end{tabular}

Then considering that the roughness factor $R_{s}$ should be 1 with ideal surface finish $\left(R_{a}=\right.$ 0 ), an exponential function curve is used to fit the data of $R_{S}$. As a result, the curve for the 
aluminum alloy is expressed as

$$
R_{S}=e^{-0.54 R_{a}}
$$

and for the medium-carbon steel:

$$
R_{S}=e^{-0.185 R_{a}}
$$

The exponential curves along with the analytical roughness factor $R_{S}$ for the aluminum alloy and medium-carbon steel are shown in Figure 3.29. It can be seen that the exponential fitting curves well reflect the correlation between $R_{s}$ and $R_{a}$.

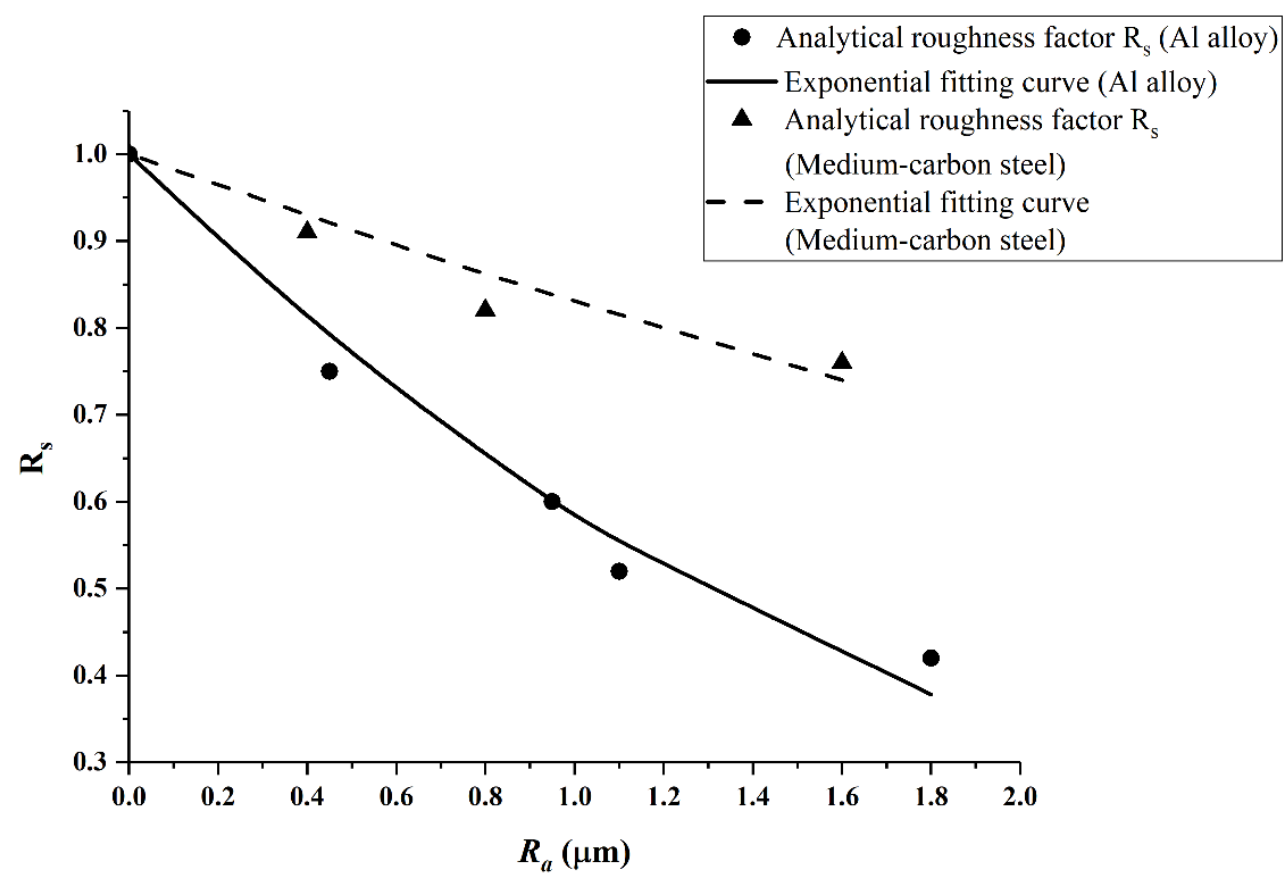

Figure 3. 29 Relationship between roughness amplitude parameter $R_{a}$ and roughness factor $R_{S}$ for aluminum alloy and medium-carbon steel. 
Likewise, according to data given in Table 3.7 and 3.8, the effect of roughness on lattice resistance $\sigma_{0}$ can be described by a linear relationship for the aluminum alloy:

$$
\sigma_{0}=-5.9249 R_{a}+185.62
$$

and for the medium-carbon steel:

$$
\sigma_{0}=-6.6071 R_{a}+330.50
$$

The linear fitting curve and analytical lattice resistance $\sigma_{0}$ against $R_{a}$ for the aluminum alloy and medium-carbon steel are presented in Figure 3.30. The comparisons display good agreement between linear fitting curve and analytical data of $\sigma_{0}$. Therefore, Eq (3.9) and Eq (3.10) have been proven to be effective expressions for $\sigma_{0}$ in terms of $R_{a}$.

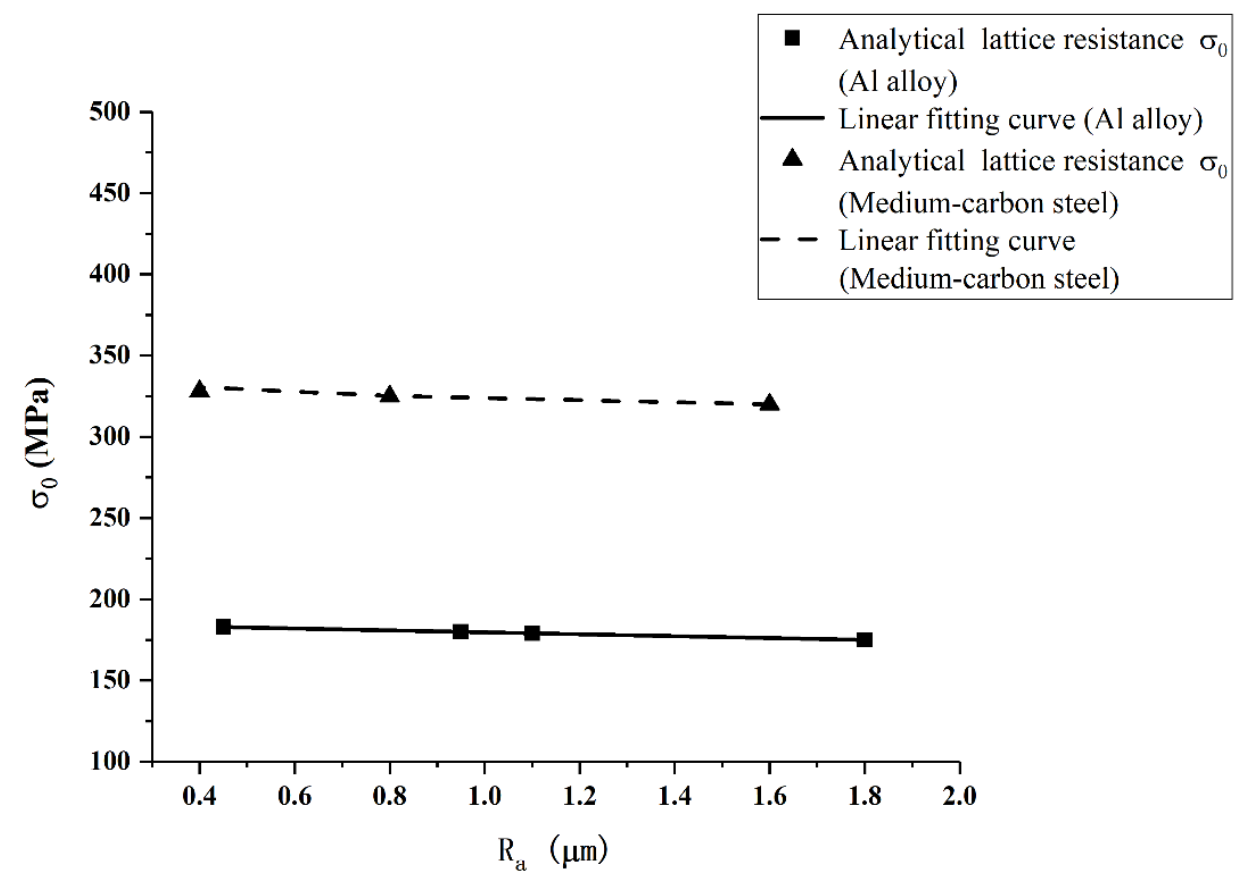

Figure 3. 30 Relationship between roughness amplitude parameter $R_{a}$ and lattice resistance $\sigma_{0}$ for aluminum alloy and medium-carbon steel. 
Finally, Eq (3.7) to Eq (3.10) are substituted into Eq (3.2), which can revise the fatigue life formula for HCF of the aluminum alloy and medium-carbon steel.: The revised figure life equation of the aluminum alloy in the HCF region is expressed as

$$
N_{c}=\frac{6 \mu w_{s} e^{-0.54 R_{a}}}{(1-v)\left(\Delta \sigma-2\left(-5.9249 R_{a}+185.62\right)\right)^{2} b_{v}}
$$

and the revised figure life of the medium-carbon steel in the HCF region is expressed as

$$
N_{c}=\frac{6 \mu w_{s} e^{-0.185 R_{a}}}{(1-v)\left(\Delta \sigma-2\left(-6.6071 R_{a}+330.50\right)\right)^{2} b_{v}}
$$

\subsubsection{Surface roughness effects on LCF}

For the remaining LCF case, as suggested in section 3.1, the strain-based formula Eq (3.1) is used to calculate the analytical fatigue life. Given that no stress-strain curves are available in literature, the effect of surface roughness on LCF is purely analyzed from the experimental data given in Table 3.9 and Table 3.10.

Table 3. 9 Experimental fatigue life data in different roughness conditions for aluminum alloy (stress amplitude of $344 \mathrm{MPa}$ )

\begin{tabular}{|c|c|}
\hline $\mathrm{R}_{\mathrm{a}}(\mu \mathrm{m})$ & $\mathrm{N}_{\mathrm{f}}$ \\
\hline 1.8 & 1250 \\
\hline 1.1 & 2100 \\
\hline 0.95 & 3800 \\
\hline 0.45 & 5500 \\
\hline
\end{tabular}


Table 3. 10 Experimental fatigue life data in different roughness conditions for mediumcarbon steel (stress amplitude of $500 \mathrm{MPa}$ )

\begin{tabular}{|c|c|}
\hline $\mathrm{R}_{\mathrm{a}}(\mu \mathrm{m})$ & $\mathrm{N}_{\mathrm{f}}$ \\
\hline 1.6 & 7174 \\
\hline 0.8 & 10498 \\
\hline 0.4 & 14348 \\
\hline
\end{tabular}

In section 3.3.3, exponential function has been found to be effective to represent the effect of roughness factor $R_{S}$ on HCF for the aluminum alloy and medium-carbon steel. It can be assumed that this function may also be applicable to the case of LCF. Thus, the expression for roughness factor $R_{S}$ can be assumed to be

$$
R_{s}=e^{\lambda R_{a}}
$$

where $\lambda$ is the exponential factor, which is derived by evaluating the relative effect of $R_{a}$ on fatigue life. This process is illustrated as follows: suppose there are two fatigue lives $N_{f}{ }^{\alpha}$ and $N_{f}{ }^{\beta}$ in different roughness conditions $R_{a}{ }^{\alpha}$ and $R_{a}{ }^{\beta}$ respectively, the relative effect of $R_{a}$ on $N_{f}$ can be evaluated:

$$
\frac{N_{f}^{\alpha}}{N_{f}{ }^{\beta}}=\frac{R_{S}^{\alpha}}{R_{S}{ }^{\beta}}=e^{\lambda\left(R_{a}^{\alpha}-R_{a}^{\beta}\right)}
$$

Then the idea is to apply multiple sets of 'relative values', $\frac{N_{f}^{\alpha}}{N_{f}{ }^{\beta}}$ and $R_{a}{ }^{\alpha}-R_{a}{ }^{\beta}$ to Eq (3.14) so that the exponent $\lambda$ can be obtained by fitting with the potential exponential relationship. Before calculation of relative values, there is a need to choose one set of data from Table 3.9 and Table 3.10 as reference data $\left(R_{a}{ }^{\beta}\right.$ and $\left.N_{f}^{\beta}\right)$. Due to the fact that the relative effect of $R_{a}$ on fatigue life $N_{f}$ is not affected by selection of reference data, in this case $R_{a}{ }^{\beta}=$ 
$0.45, N_{f}^{\beta}=5500 . R_{a}^{\alpha}$ and $N_{f}^{\alpha}$ represent either set of data from Table 3.9 and Table 3.10. Take an example for illustration, when $R_{a}{ }^{\alpha}=0.45$ and ${N_{f}}^{\alpha}=5500$, the 'relative values' $\frac{N_{f}^{\alpha}}{N_{f}{ }^{\beta}}$ and $R_{a}^{\alpha}-R_{a}^{\beta}$ for the aluminum alloy can be calculated to be 1 and 0 , respectively. Following the procedures described above, 4 and 3 sets of 'relative values' for aluminum alloy and medium-carbon steel are calculated and presented in Table 3.11 and Table 3.12, respectively.

Table 3. 11 Relative values used for determination of $\lambda$ for aluminum alloy

\begin{tabular}{|c|c|c|c|c|c|}
\hline $\mathrm{R}_{\mathrm{a}}^{\alpha}(\mu \mathrm{m})$ & $\mathrm{R}_{\mathrm{a}}^{\beta}(\mu \mathrm{m})$ & $\mathrm{N}_{\mathrm{f}}^{\alpha}$ & $\mathrm{N}_{\mathrm{f}}^{\beta}$ & $\mathrm{N}_{\mathrm{f}}^{\alpha} / \mathrm{N}_{\mathrm{f}}^{\beta}$ & $\mathrm{R}_{\mathrm{a}}^{\alpha}-\mathrm{R}_{\mathrm{a}}{ }^{\beta}$ \\
\hline 0.45 & \multirow{4}{*}{0.45} & 5500 & \multirow{4}{*}{5500} & 1 & 0 \\
\hline 0.95 & & 3800 & & 0.691 & 0.5 \\
\hline 1.1 & & 2100 & & 0.382 & 0.65 \\
\hline 1.8 & & 1250 & & 0.227 & 1.35 \\
\hline
\end{tabular}

Table 3. 12 Relative values used for determination of $\lambda$ for medium-carbon steel

\begin{tabular}{|c|c|c|c|c|c|}
\hline $\mathrm{R}_{\mathrm{a}}^{\alpha}(\mu \mathrm{m})$ & $\mathrm{R}_{\mathrm{a}}^{\beta}(\mu \mathrm{m})$ & $\mathrm{N}_{\mathrm{f}}^{\alpha}$ & $\mathrm{N}_{\mathrm{f}}^{\beta}$ & $\mathrm{N}_{\mathrm{f}}^{\alpha} / \mathrm{N}_{\mathrm{f}}^{\beta}$ & $\mathrm{R}_{\mathrm{a}}{ }^{\alpha}-\mathrm{R}_{\mathrm{a}}{ }^{\beta}$ \\
\hline 0.4 & \multirow{3}{*}{0.4} & 14348 & \multirow{3}{*}{14348} & 1 & 0 \\
\hline 0.8 & & 10498 & & 0.732 & 0.4 \\
\hline 1.6 & & 7174 & & 0.5 & 1.2 \\
\hline
\end{tabular}

All relative values are then fitted by $\mathrm{Eq}(3.14)$ and $\lambda$ is determined to be -1.13 for the aluminum alloy and -0.56 for medium-carbon steel. The effectiveness of fitting is examined in Figure 3.31. The results show that the exponential fitting well coincides with the analytical data, hence, the expression of $R_{S}$ for LCF of the aluminum alloy can be given as:

$$
R_{S}=e^{-1.13 R_{a}}
$$


and for the medium-carbon steel:

$$
R_{S}=e^{-0.56 R_{a}}
$$

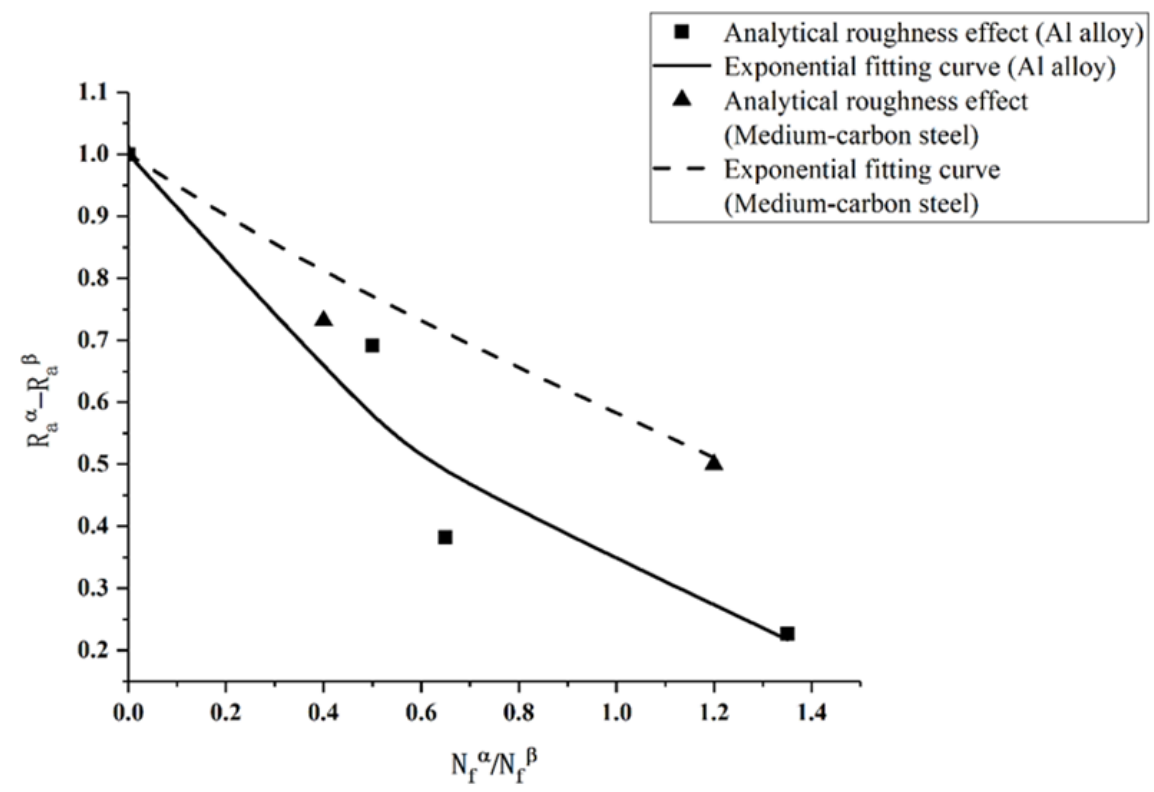

Figure 3. 31 Determination of exponential factor $\lambda$ for aluminum alloy and mediumcarbon steel.

Once again, Eq (3.1) can be revised by substituting Eq (3.15) and Eq (3.16) into Eq (3.1)

for aluminum alloy as

$$
N_{c}=\frac{8(1-v) w_{S} e^{-1.13 R a}}{3 \mu b_{v}} \frac{1}{\Delta \varepsilon_{p}{ }^{2}}
$$

Similarly, for medium-carbon steel, the revised figure life equation of the medium-carbon steel in the LCF region is expressed as 


$$
N_{c}=\frac{8(1-v) w_{S} e^{-0.56 R a}}{3 \mu b_{v}} \frac{1}{\Delta \varepsilon_{p}^{2}}
$$

The results shown in section 3.3 provide some reference significance for engineering application since surface roughness of most engineering components belongs to the range of 0.2 to $1.8 \mu \mathrm{m}$, which has been covered in this study. However, it is noteworthy that the surface roughness effect on fatigue life can vary from material to material. The meaning of the correlation between surface roughness and the TMW model parameters proposed in this study is to give an idea for quantification of component surface roughness effect. 


\section{Chapter 4 FEA Simulation of Fatigue Crack Nucleation}

In Chapter 3, the Tanaka-Mura-Wu (TMW) model has shown to be capable of predicting the fatigue life of engineering materials with known elastic modulus, Burgers vector, surface energy, and cyclic plastic strain range under coupon testing conditions. However, microstructural inhomogeneity in grain size and orientation distribution always exist in real engineering materials, which may contribute to fatigue life scatter. The assessment of fatigue life scatter is a significant part of fatigue design, in order to keep the probability of fatigue life prediction failure low. This requires a large amount of fatigue testing every time when the material manufacturing process (or vendor) is changed. Therefore, the incentive of understanding the effects of material microstructural variability is very high through numerical simulation and analysis, which will save huge amounts of money and time for product design with assured durability.

In this chapter, finite element microstructure modeling is conducted to assess the fatigue crack nucleation life of Haynes 282 alloy at different strain amplitudes from LCF to HCF. A material representative volume element (RVE) is created using Voronoi tessellation method, and 20 random grain orientation distribution functions are assigned to the material RVE to evaluate the variation of fatigue crack nucleation life in both HCF and LCF simulations. Finally, the predicted fatigue crack nucleation life of Haynes 282 and the scattering of the simulated life are compared with the experimental data from literature for 
Haynes 282 alloy. The effect of grain orientation on the fatigue crack nucleation life is thus evaluated.

This chapter includes all aspects of the microstructural Finite Element Analysis (FEA) modeling for Haynes 282 alloy. First, the microstructural characteristics of the objective material for LCF and HCF simulations are introduced in section 4.1. Then, the details of microstructure modeling are presented in section 4.2 and the FEM analysis techniques associated with finite element code-Abaqus/Standard are introduced in section 4.3. Section 4.4 describes the material properties of the FEA model including anisotropic elasticity and plasticity constants. Simulation of hysteresis behavior of Haynes 282 alloy is also analyzed in this section. The procedures of fatigue nucleation life prediction for Haynes 282 alloy using the combined approach of the TMW model with the microstructural FEA simulation are given in section 4.5. Finally, the results of the LCF and HCF life predictions of Haynes 282 alloy are analyzed and discussed in section 4.6.

\subsection{Objectives of FEA Simulation}

In this study, Ni-base superalloy Haynes 282 is chosen as the objective material of FEA simulation, because it is a promising candidate material for A-USC steam turbines and its fatigue test data are available in literature. The details of the simulation model such as material properties, microstructure and loading conditions are required to be in consistency with that used in the previous studies [84] [86] so that the predicted LCF life and HCF life 
of Haynes 282 can be comparable with the experimental data reported from the previous studies.

\subsubsection{Reference for LCF simulation}

The LCF simulation aims at the LCF testing conducted on Haynes 282 by He et al. [84]. The Haynes 282 material used in their work was extracted from the rim and core sections of a large forged ingot. The chemical composition of the material is the same as given in Table 2.3. The SEM image of fatigue cracks of the material in its peak-aged condition is shown in Figure 4.1. Four fatigue tests were performed on the alloy at room temperature according to the ASTM E606 [6] and ISO 12106 [104] in strain-controlled mode with a triangular wave loading. The strain ranges were full reversed with magnitudes of $0.84 \%$, $0.93 \%, 1.34 \%$ and $1.70 \%$, but only strain ranges of $0.84 \%$ and $0.93 \%$ are considered for LCF simulation in the current study. The stabilized hysteresis loops of LCF at room temperature for the rim material are shown in Figure 4.2 and they are used to calibrate the material behavior model of Haynes 282 for LCF case study. 


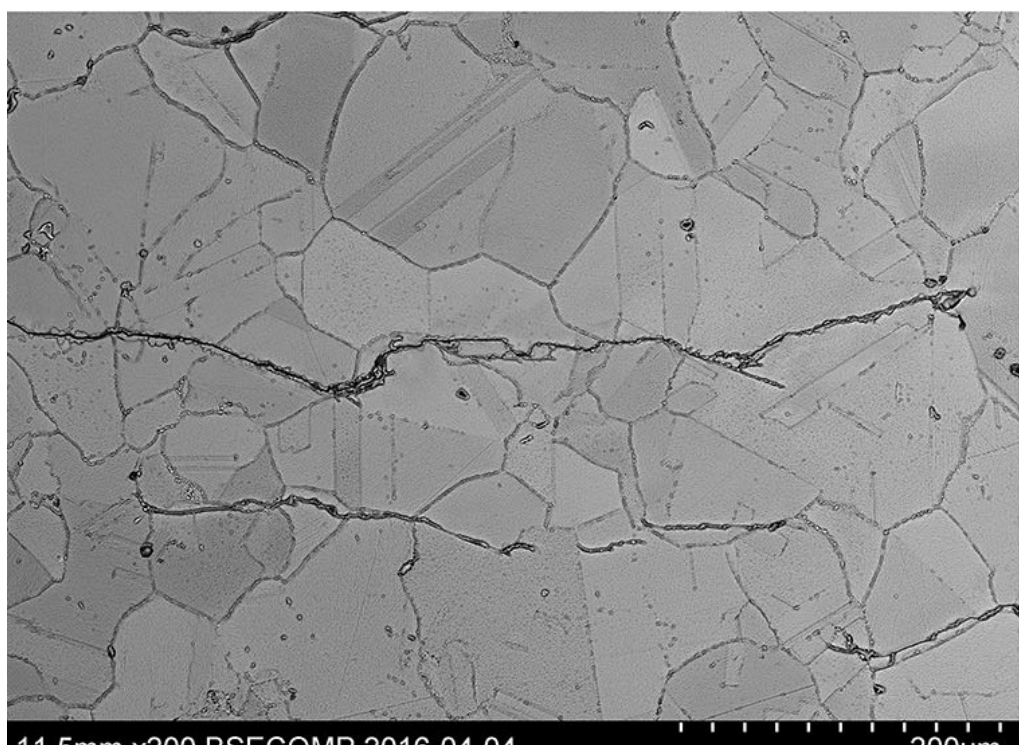

11.5mm x200 BSECOMP 2016-04-04

200um

Figure 4. 1 SEM image of fatigue cracks in Haynes 282 microstructure for LCF simulation [84].

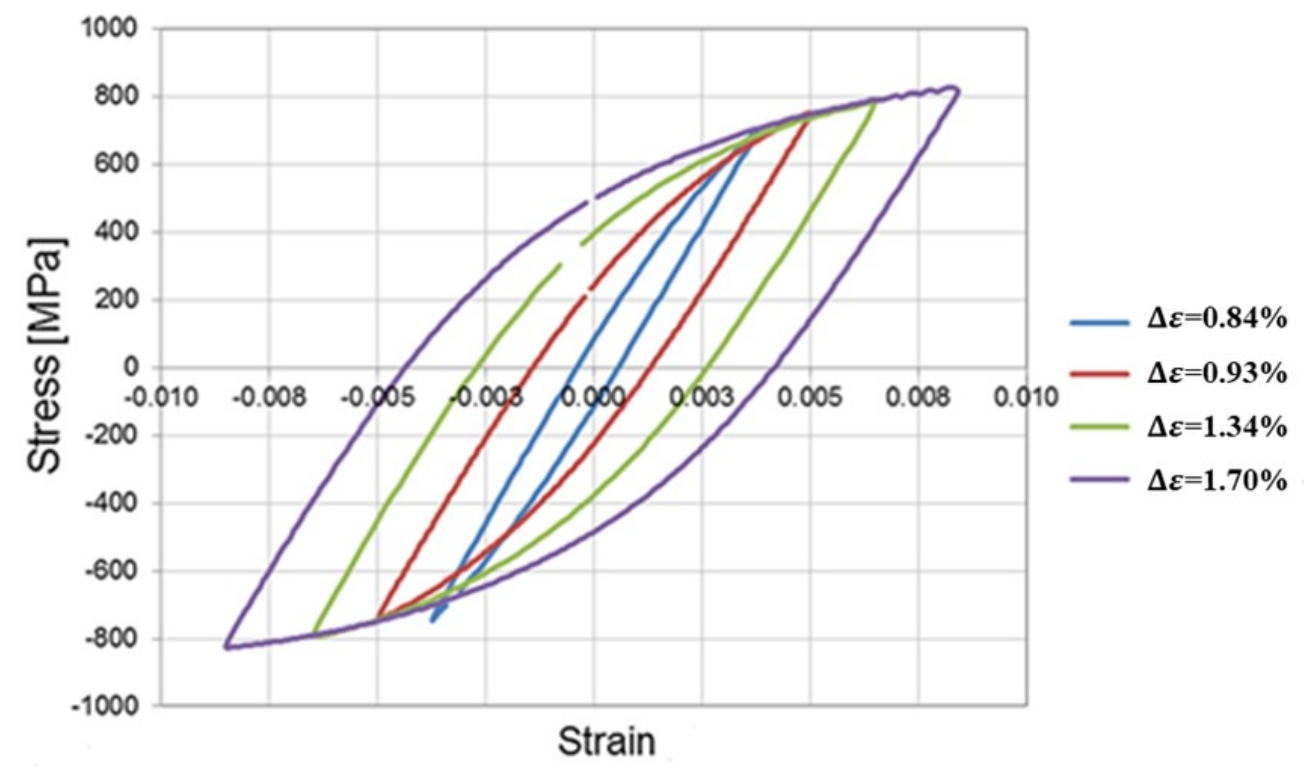

Figure 4. 2 Stabilized hysteresis loops of Haynes 282 under LCF at room temperature [84]. 


\subsubsection{Reference for HCF simulation}

The samples of Haynes 282 for HCF simulation are rods processed by standard heat treatment [86]: solution treatment at $1120-1150{ }^{\circ} \mathrm{C}$ followed by water cooling; first aging at $1010{ }^{\circ} \mathrm{C}$ for 2 hours followed by air cooling; second aging at $788{ }^{\circ} \mathrm{C}$ for 8 hours followed by air cooling. The optical microstructure of the material is shown in Figure 4.3. Stresscontrolled HCF tests were conducted at room temperature based on the Chinese standard GB/T 3075 [105]. The stresses were fully reversed with ranges of $415 \mathrm{MPa}, 400 \mathrm{MPa}, 380$ $\mathrm{MPa}$ and $370 \mathrm{MPa}$, but only stress ranges of $415 \mathrm{MPa}$ and $400 \mathrm{MPa}$ are considered for FEA simulation of the HCF case in the present study.

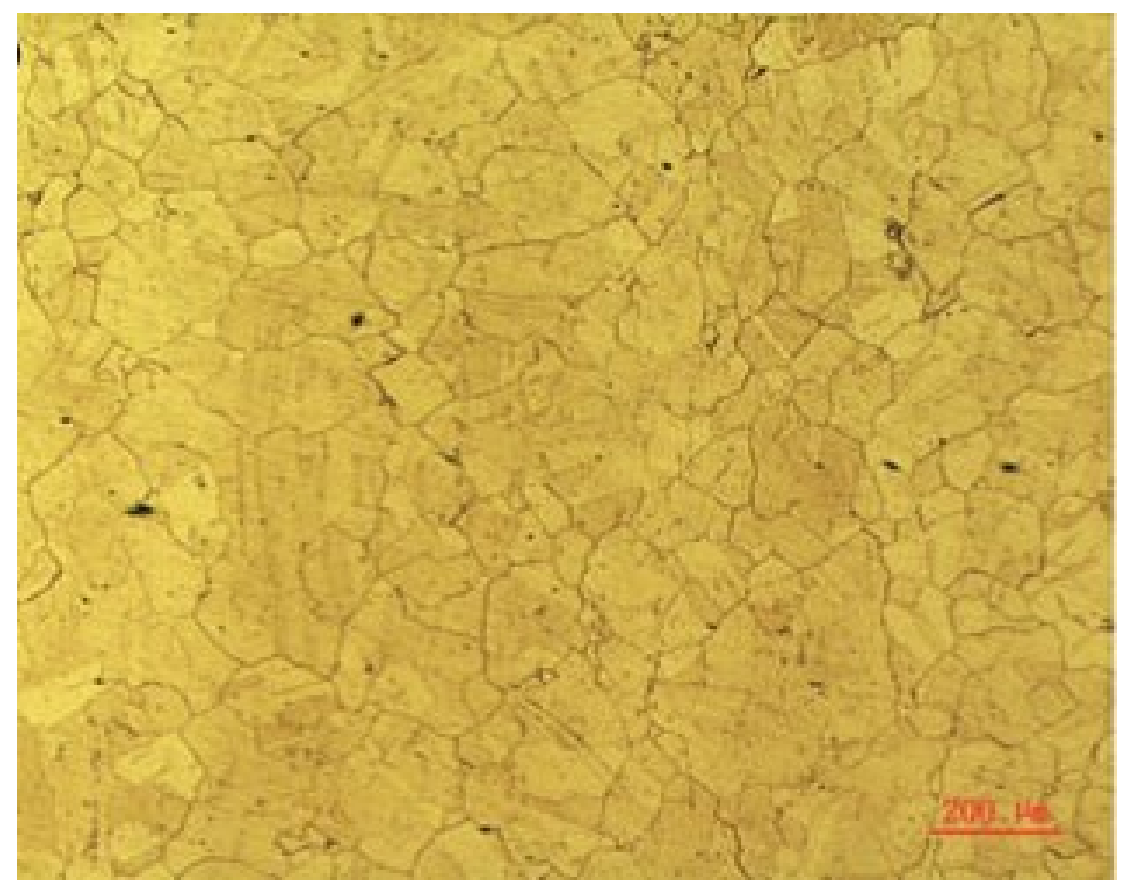

Figure 4. 3 Optical image of Haynes 282 microstructure for HCF simulation [86]. 


\subsection{Microstructure Model}

It is widely recognized that the properties of polycrystalline materials highly depend on the microstructural characteristics at grain level such as grain size, grain shape and grain orientation. The heterogeneous nature of grain microstructure enables polycrystalline materials to exhibit anisotropic response during cyclic deformation. In order to appropriately simulate the fatigue crack nucleation of Haynes 282 alloy, a three dimensional (3D) representative volume element (RVE) is constructed in this study to represent the microstructure model. In this section, the procedures to generate the 3D RVE model for fatigue nucleation simulation are described in detail.

\subsubsection{D RVE model}

As introduced in Chapter 2, an RVE is a periodic unit cell taken from polycrystalline materials and it possesses the same grain structure as the bulk materials. RVEs have been widely used in numerical analyses at mesoscopic level, because they represent the heterogeneous materials in a simplified geometry. The 3D RVE model used in this study is to represent a grain aggregate from a fatigue coupon of Haynes 282, as shown in Figure 4.4. It is generated through Voronoi tessellation, which is performed using Python Scripts, as given in Appendix A. The general procedures to generate 3D RVE model are divided into three main steps: i) Determine RVE size; ii) Locate Voronoi generators; iii) Process the model. 


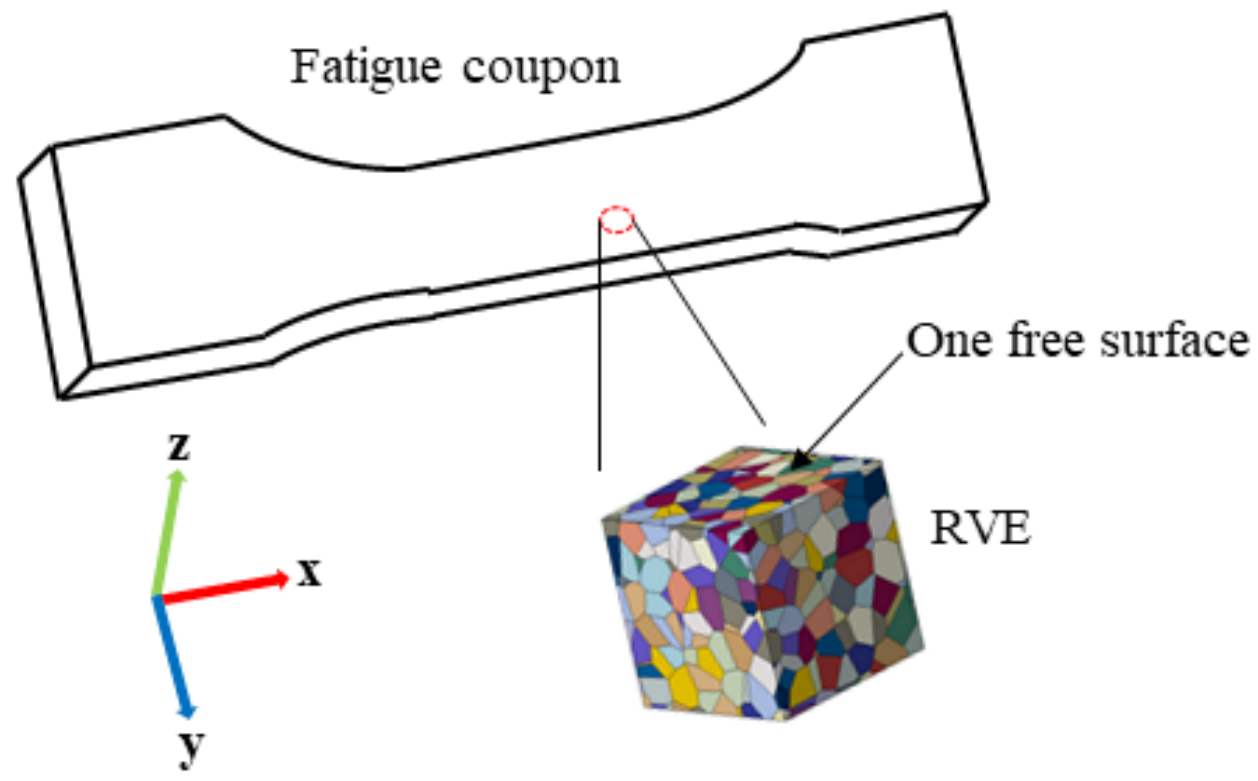

Figure 4. 4 Illustration of RVE for Haynes 282.

In general, a grain structure generated by Voronoi tessellation can be extended to infinity in $3 \mathrm{D}$ space. Therefore, the size of a cubic RVE needs to be determined for the interest of the study. There are two considerations with respect to the RVE size: One is that the RVE size needs to be large enough so that it includes a sufficient number of grains to represent the global material behavior, the other is that it should also be small enough for computational efficiency. According to the study by Brommesson et al. [106], 500 grains were found to be fairly good to model the stress response of Haynes 282 alloy under uniaxial loading as observed in the experiment. Consequently, the number of grains contained in the 3D RVE model is not less than 500, thus, 625 grains are used in the RVE model. Grain size is another factor influencing the size of an RVE model. The grain size of the RVE model in the present study is taken according to the SEM and optical images, 
Figure 4.1 for LCF [84] and Figure 4.3 for HCF [86]. By applying the intercept procedure [107], the approximate average diameter of grains in Figure 4.1 and Figure 4.3 are estimated to be $82.18 \mu \mathrm{m}$ and $71.78 \mu \mathrm{m}$, respectively. Thus, the average grain size is determined to be approximately $73 \mu \mathrm{m}$ for Haynes 282 alloy in the present study. Based on all above considerations, the RVE size is finally decided to be $500 \times 500 \times 500 \mu \mathrm{m}^{3}$.

For tessellation, the Voronoi generators need to be located within the domain of an RVE first, to form polyhedrons which are also known as Voronoi regions. Each Voronoi generator corresponds to one Voronoi region in the RVE model so that the total number of Voronoi generators should be equal to the number of grains in the RVE domain. In this case, 625 Voronoi generators are randomly distributed in the RVE model. Actually, in order to fill the RVE without voids, some Voronoi generators are placed outside of the RVE boundary and also inside, then the RVE model is trimmed to remove the irregular boundary cells. All aforementioned steps for building the 3D RVE model are programmed into Python Scripts. Finally, as presented in Figure 4.5, a cubic $\left(500 \times 500 \times 500 \mu \mathrm{m}^{3}\right)$ 3D RVE model with total 625 grains are created by running the Python Scripts (given in Appendix A). 


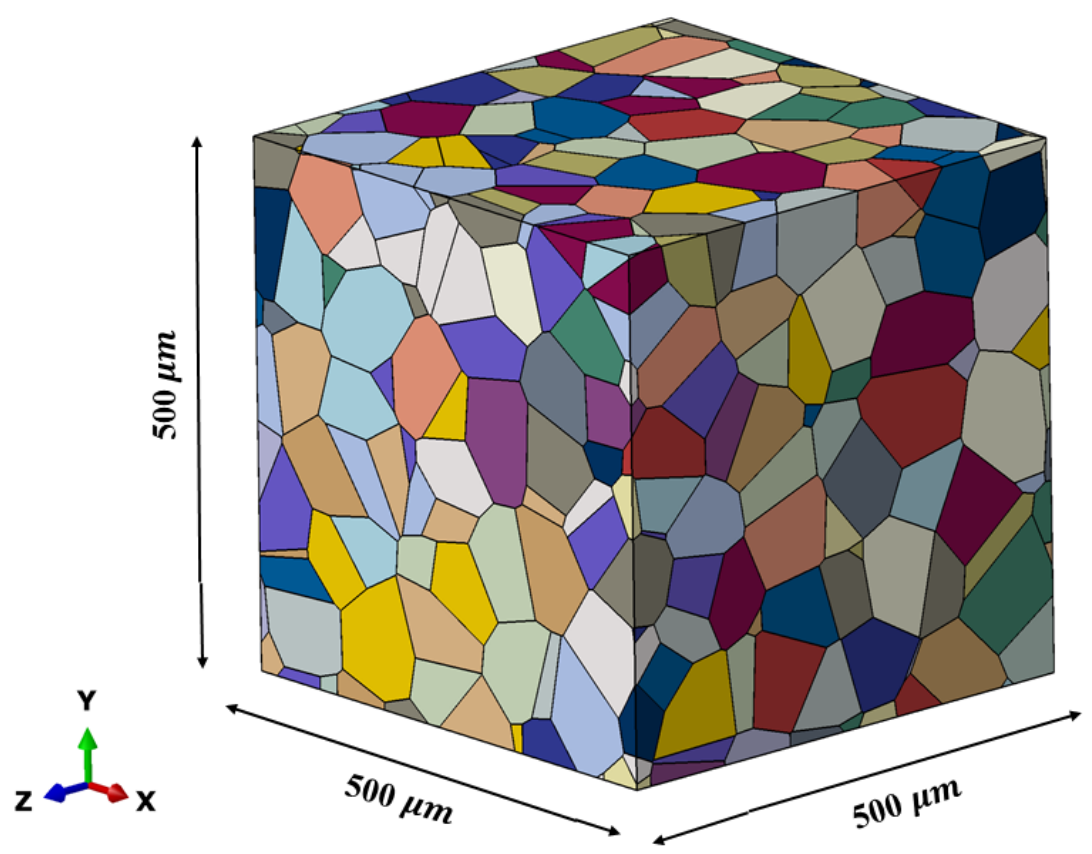

Figure 4. 5 3D RVE model of Haynes 282 for microstructure FEA simulation.

\subsubsection{Grain orientation and inverse pole figure}

In order to characterize the effect of grain orientation in the fatigue life of Haynes 282, grain orientations with 20 random distribution functions are assigned to individual grains in the 3D RVE model for $20 \mathrm{LCF}$ and $20 \mathrm{HCF}$ simulations. In Abaqus, the grain orientation is created by introducing a local coordinate system within each grain. As illustrated in Figure 4.6, the procedure includes to define an origin $\mathrm{O}$ of local coordinate first, followed by assigning a location of point $A\left(x_{1}, y_{1}, z_{1}\right)$ with $O A$ as the $x^{\prime}$ axis, and location of another point $B\left(\mathrm{x}_{2}, \mathrm{y}_{2}, \mathrm{z}_{2}\right)$ to define the $x^{\prime} y^{\prime}$ plane in the local coordinate system. The points of $A$ $\left(x_{1}, y_{1}, z_{1}\right)$ and point $B\left(\mathrm{x}_{2}, \mathrm{y}_{2}, \mathrm{z}_{2}\right)$ are randomly selected in the range of $[-1,1]$ by using the Python Scripts given in Appendix B. Then, the directions of $x^{\prime}, y^{\prime}, z^{\prime}$ axes of local coordinate system are determined, and their miller index notations are recorded. 


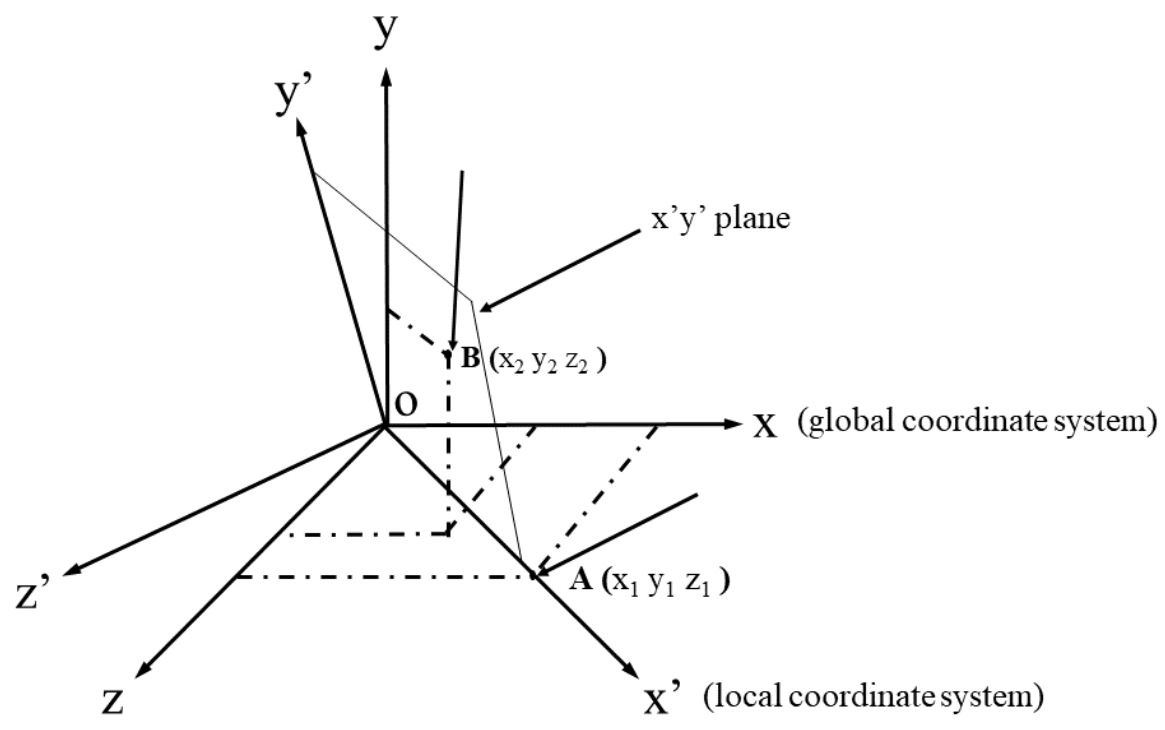

Figure 4. 6 Definition of grain orientation.

The grain orientation in a 3D space is usually represented by the Bunge Euler angles $\varphi, \theta$, and $\psi$, which are defined through the following sequence of counterclockwise rotations of the crystal frame (local coordinate system) directions: first around the fixed $z$ ' axis, second around the rotated $x^{\prime}$ axis and finally around the rotated $z^{\prime}$ axis, as illustrated in Figure 4.7.

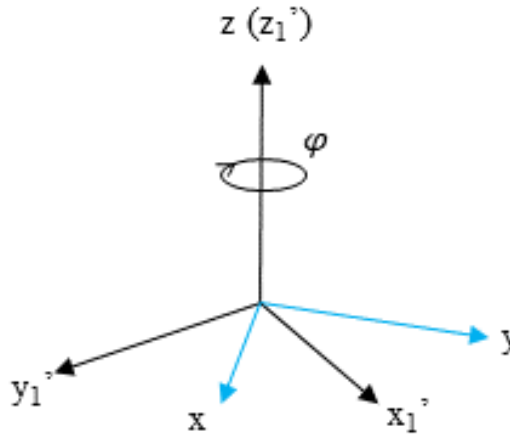

(a)

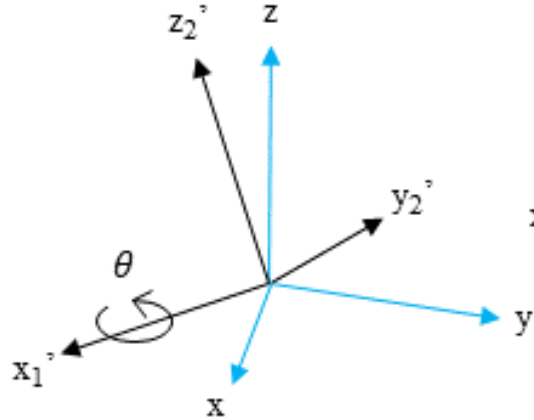

(b)

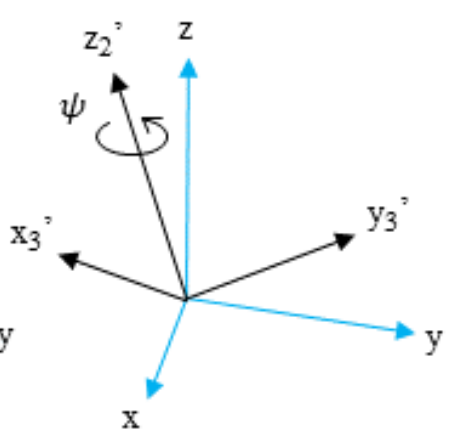

(c)

Figure 4. 7 Illustration of crystal frame rotation about (a) $z$ axis (first), (b) rotated $x$ axis (second) and (c) rotated $z$ axis (third). 
Grain orientations are also often presented in inverse pole figure, as experimentally determined using electron backscatter diffraction (EBSD) technique. A Matlab program using a toolbox named mtex has been developed to analyze grain orientations, as given in Appendix C. The description of grain orientation by Matlab code requires input of three Euler angles. Therefore, the directions of $x^{\prime}, y^{\prime}$ and $z^{\prime}$ axes of the local coordinate system in Abaqus are introduced to Matlab to calculate the corresponding Euler angles. Finally, grain orientations are automatically plotted in the inverse pole figures using mtex.

\subsection{FEA Model}

Once the Voronoi tessellation is implemented and grain orientations are assigned within the RVE, the next step is to impose appropriate boundary conditions and develop meshing for the FEA simulation. Boundary conditions, element type and mesh density are selected to truthfully represent the material element behavior, yet computationally efficient to achieve the required results.

\subsubsection{Boundary conditions}

The boundary conditions are applied to the REV model to simulate the meso-scale uniform strain condition, i.e., the entire RVE deforms with a uniform strain. Within the RVE, however, the stress-strain distribution may not be uniform at the microstructure scale, within each grain and between neighboring grains. For uniform meso-strain, usually, a 
linear displacement boundary condition is applied at one end of the RVE [68].

For simplicity, the boundary condition of uniaxial loading is used in the present study. The displacements of nodes in the $x$ direction on the plane $x=0$ are all constrained to zero, while the traction stresses in other directions are $0\left(t_{y}=t_{z}=0\right)$ on the surface of the RVE. The displacement of uniform amplitude in the $x$ direction is applied to all nodes on the boundary plane $x=500 \mu \mathrm{m}$. For the LCF case study, the displacement amplitudes are limited to $4.2 \mu \mathrm{m}$ and $4.65 \mu \mathrm{m}$ thus to obtain the strain amplitudes of $0.84 \%$ and $0.93 \%$. The HCF strain amplitude is calculated as the applied stress (415 MPa and $400 \mathrm{MPa}$ ) is divided by the Young's modulus of Haynes 282. To eliminate rigid motion, the node with $x=0, y=0, z=500(\mu \mathrm{m})$ is completely fixed and the node with $x=y=z=0$ is only allowed to move along the $z$ axis. In addition, the nodes on other surfaces are all traction-free ( $t=$ 0). The boundary conditions can be expressed by a piecewise equation:

$$
\left\{\begin{array}{c}
d_{x}=0, \quad t_{y}=t_{z}=0(\mathrm{x}=0) \\
d_{x}=\frac{\Delta \varepsilon d_{R}}{2}, t_{y}=t_{z}=0(\mathrm{x}=500) \\
d_{x}=d_{y}=d_{z}=0(\mathrm{x}=\mathrm{y}=0, \mathrm{z}=500) \\
d_{x}=d_{y}=0(\mathrm{x}=\mathrm{y}=\mathrm{z}=0) \\
t=0(\text { Otherwise })
\end{array}\right.
$$

where $d$ represents displacement, $t$ represents traction, $\Delta \varepsilon$ is strain range and $d_{R}$ is the edge length of RVE. Among the applied boundary conditions, there are four free surfaces of the RVE. In a real engineering component, a small material element such as a RVE can only have one free surface and the other three surfaces are supposed to be connected to the bulk 
materal. However, this cannot be realized for a stand alone RVE because the connecting boundary conditions are difficult to prescribe, truthfully. Therefore, for the purpose of comparison in evaluating grain orientation effects while keeping the same geometrical grain structure, one free surface, $z=0$ plane, is selected as the representative free surface of the material.

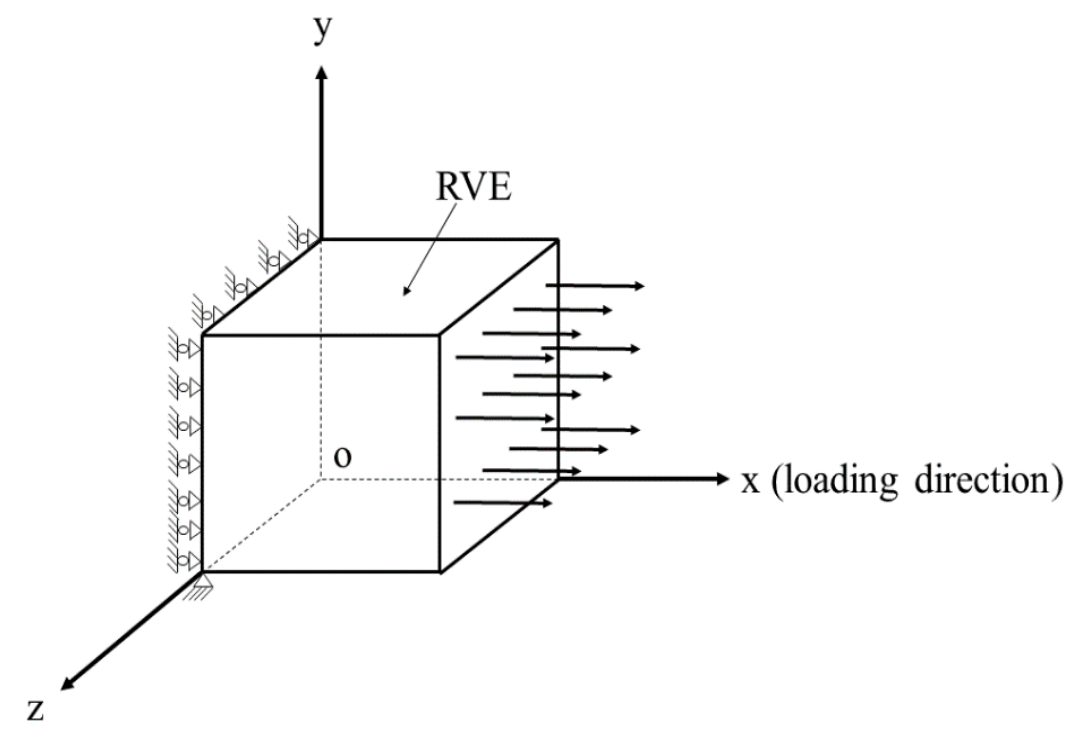

Figure 4. 8 Illustration of boundary conditions of the RVE model.

\subsubsection{Element and mesh}

The meshing strategy includes selecting appropriate element type and meshing density for the FEM model, which has a considerable influence on the simulation results. Due to the geometrical complexity of the Voronoi grain structure, it is extremely difficult to mesh the RVE model with hexahedral elements. Therefore, free meshing technique is employed with the 4-node linear tetrahedral elements. 
Normally, the accuracy of numerical results can be improved with increasing mesh density, but a very fine mesh will introduce an enormous amount of degrees of freedom, which requires a large computing power. To reach an optimal compromise of computing accuracy and time, a convergence study has been carried out. Four different meshes are used for elastic stress analyses of the RVE. The number of elements used in these meshes are $252571,511658,1346296$, and 2596633 with corresponding meshing sizes of $\sim 17,13,9$ and $7 \mu \mathrm{m}$. Figure 4.9 shows the variation of Von Mises stress along the $x$-axis at the edge of $y=z=500 \mu \mathrm{m}$, obtained from the four meshes. It can be seen that large difference exists between the results from coarser meshes with 252571 and 511658 elements and fine meshes with 1346296 and 2596633 elements. When the number of elements is increased to 1346296 , the Von Mises stresses start to converge at almost all nodal positions. Beyond this point, extra computing cost due to doubling of element quantities from 1346296 to 2596633 does not seem to be paid off with any significant increase in accuracy. Consequently, the 1346296-element mesh is used in the subsequent fatigue crack nucleation simulations of Haynes 282. The mesh of the RVE is shown in Figure 4.10. 


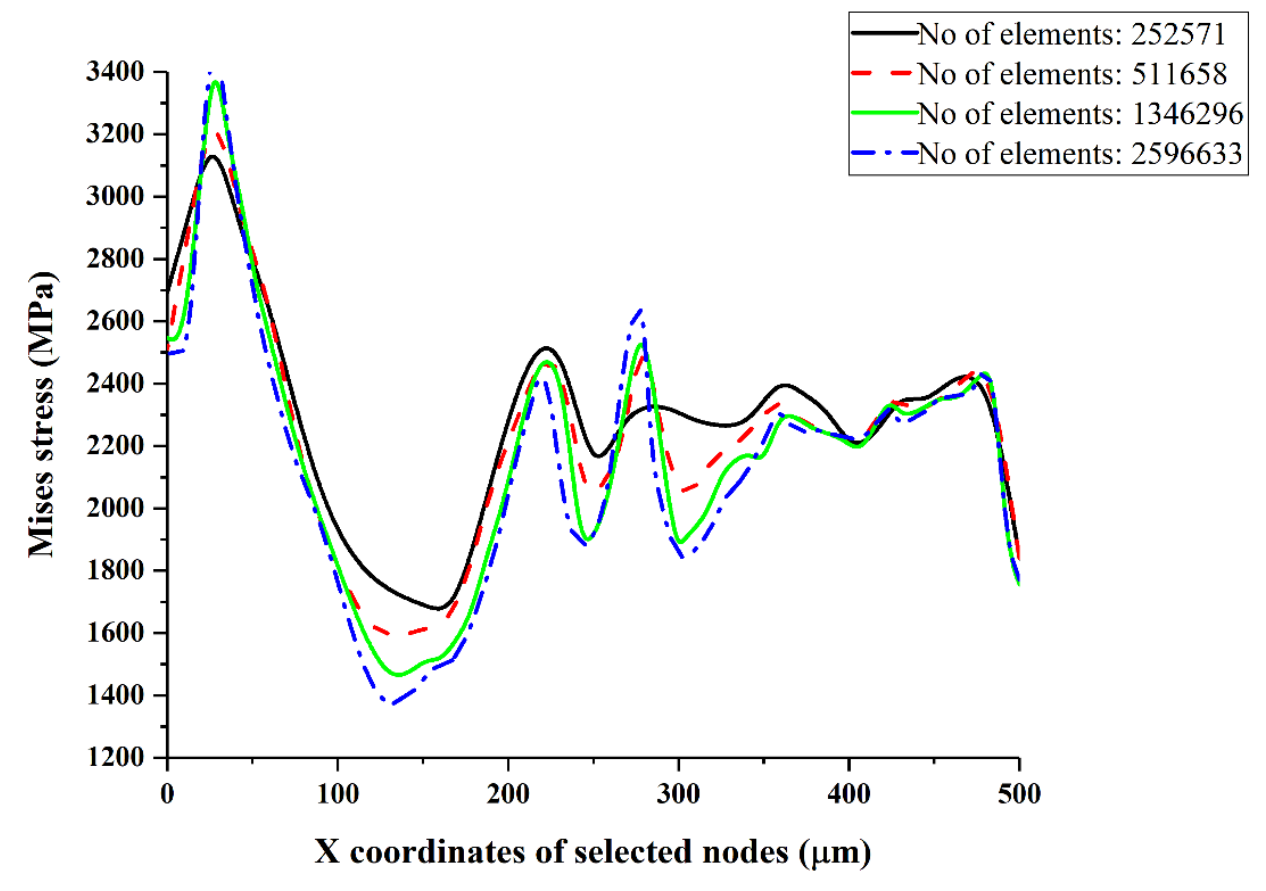

Figure 4. 9 Convergence study with four different meshes.

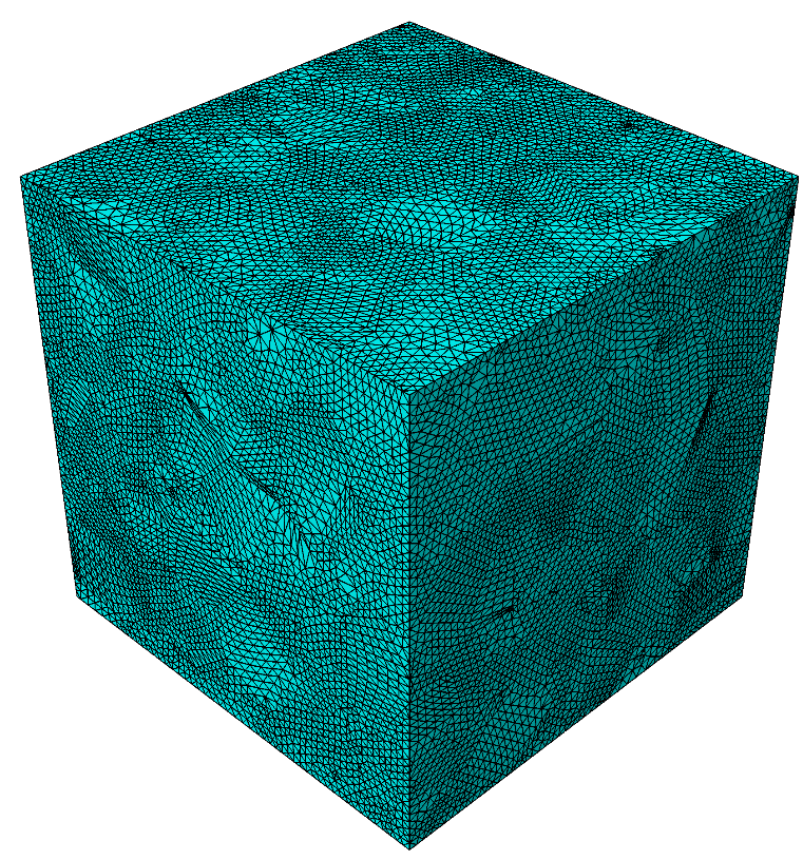

Figure 4. 10 Meshed model of the RVE. 


\subsection{Material Properties}

In an RVE, material behavior is modeled at grain level to faithfully represent the heterogeneous nature of the microstructure. Crystallites in a polycrystalline material possess anisotropic properties including anisotropic elasticity and anisotropic yielding, which highly depend on grain orientation. In the present study, anisotropic elasticity is considered for the elastic behavior, while Hill's yielding surface and kinematic hardening are employed to describe the plastic behavior of Haynes 282 .

\subsubsection{Anisotropic elastic constants}

Since Haynes 282 is a nickel-based superalloy with a crystalline structure of cubic symmetry, the anisotropic elasticity tensor can reduce from 36 constants to 3 for orthotropic elasticity:

$$
\left[\begin{array}{l}
\sigma_{11} \\
\sigma_{22} \\
\sigma_{33} \\
\sigma_{23} \\
\sigma_{12} \\
\sigma_{13}
\end{array}\right]=\left[\begin{array}{cccccc}
C_{11} & C_{12} & C_{13} & 0 & 0 & 0 \\
C_{12} & C_{22} & C_{23} & 0 & 0 & 0 \\
C_{13} & C_{23} & C_{33} & 0 & 0 & 0 \\
0 & 0 & 0 & C_{44} & 0 & 0 \\
0 & 0 & 0 & 0 & C_{55} & 0 \\
0 & 0 & 0 & 0 & 0 & C_{66}
\end{array}\right] *\left[\begin{array}{c}
\varepsilon_{11} \\
\varepsilon_{22} \\
\varepsilon_{33} \\
\varepsilon_{23} \\
\varepsilon_{12} \\
\varepsilon_{13}
\end{array}\right]
$$

with $C_{11}=C_{22}=C_{33}, C_{12}=C_{13}=C_{23}$, and $C_{44}=C_{55}=C_{66}$.

Considering that the elastic constants of nickel-based single crystal superalloys are almost identical, the values for nickel-based single crystal PWA 1480 at $100{ }^{\circ} \mathrm{F}$ [68] are assumed suitable for Haynes 282 grains, i.e. $C_{11}=C_{22}=C_{33}=250 \mathrm{GPa}, C_{12}=C_{13}=C_{23}=163 \mathrm{GPa}$, 
$C_{44}=C_{55}=C_{66}=129 \mathrm{GPa}$.

\subsubsection{Plasticity parameters}

Metallic materials often exhibit nonlinear stress-strain behaviors, e.g., tensile curves. The turning point from the initial linear elastic behavior defines the yielding stress, beyond which, the material enters plastic behavior regime. In this subsection, the anisotropic plasticity parameters-Hill potential constants will be determined.

\subsubsection{Transformation of stresses}

To determine the Hill's potential constants from uniaxial tensile tests, first, the global stress needs to be transformed into the material's cubic axis system. Let the $\{\mathrm{x}, \mathrm{y}, \mathrm{z}\}$ represents the global coordinate system, as in the FEM or fatigue testing, and $\left\{\mathrm{x}^{\prime}, \mathrm{y}^{\prime}, \mathrm{z}^{\prime}\right\}$ represents the local coordinate system. The relation between the two coordinate systems is illustrated in Figure 4.11. 


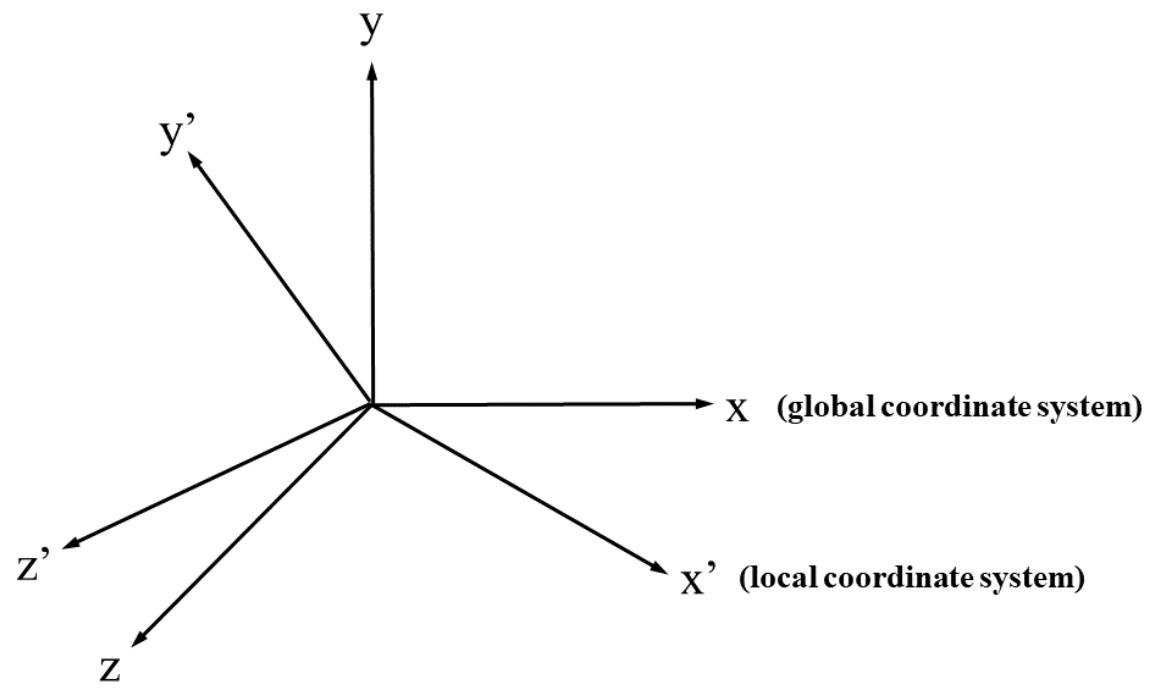

Figure 4. 11 Definitions of two coordinate systems.

By the Cartesian coordinate transformation rule, the relation of the stress in local coordinate system to the global coordinate system can be expressed in matrix form:

$$
\left[\sigma^{\prime}\right]=[Q][\sigma]
$$

where $[\sigma]$ and $\left[\sigma^{\prime}\right]$ are stress vectors in the global and local coordinate system, respectively, and $[Q]$ is the transformation matrix. In a $3 \mathrm{D}$ space, they are often expressed in the following form:

$$
[\sigma]=\left[\begin{array}{c}
\sigma_{x} \\
\sigma_{y} \\
\sigma_{z} \\
\sigma_{x y} \\
\sigma_{y z} \\
\sigma_{x z}
\end{array}\right] \text { and }\left[\sigma^{\prime}\right]=\left[\begin{array}{c}
\sigma_{x^{\prime}} \\
\sigma_{y^{\prime}} \\
\sigma_{z^{\prime}} \\
\sigma_{x y^{\prime}} \\
\sigma_{y z^{\prime}} \\
\sigma_{x z^{\prime}}
\end{array}\right]
$$

The transformation matrix $[Q]$ is given by 


$$
[Q]=\left[\begin{array}{cccccc}
\alpha_{1}{ }^{2} & \alpha_{2}{ }^{2} & \alpha_{3}{ }^{2} & 2 \alpha_{3} \alpha_{2} & 2 \alpha_{1} \alpha_{3} & 2 \alpha_{2} \alpha_{1} \\
\beta_{1}{ }^{2} & \beta_{2}{ }^{2} & \beta_{3}{ }^{2} & 2 \beta_{3} \beta_{2} & 2 \beta_{1} \beta_{3} & 2 \beta_{2} \beta_{1} \\
\gamma_{1}{ }^{2} & \gamma_{2}{ }^{2} & \gamma_{3}{ }^{2} & 2 \gamma_{3} \gamma_{2} & 2 \gamma_{1} \gamma_{3} & 2 \gamma_{2} \gamma_{1} \\
\beta_{1} \gamma_{1} & \beta_{2} \gamma_{2} & \beta_{3} \gamma_{3} & \left(\beta_{2} \gamma_{3}+\beta_{3} \gamma_{2}\right) & \left(\beta_{1} \gamma_{3}+\beta_{3} \gamma_{1}\right) & \left(\beta_{1} \gamma_{2}+\beta_{2} \gamma_{1}\right) \\
\gamma_{1} \alpha_{1} & \gamma_{2} \alpha_{2} & \gamma_{3} \alpha_{3} & \left(\gamma_{2} \alpha_{3}+\gamma_{3} \alpha_{2}\right) & \left(\gamma_{1} \alpha_{3}+\gamma_{3} \alpha_{1}\right) & \left(\gamma_{1} \alpha_{2}+\gamma_{2} \alpha_{1}\right) \\
\alpha_{1} \beta_{1} & \alpha_{2} \beta_{2} & \alpha_{3} \beta_{3} & \left(\alpha_{2} \beta_{3}+\alpha_{3} \beta_{2}\right) & \left(\alpha_{1} \beta_{3}+\alpha_{3} \beta_{1}\right) & \left(\alpha_{1} \beta_{2}+\alpha_{2} \beta_{1}\right)
\end{array}\right]
$$

where $\alpha, \beta$ and $\gamma$ are direction cosines between the axes in two coordinate systems. For a single crystal under uniaxial tensile loading in a direction of Miller indices $[h k l]$, as defined in local coordinate system, the global stress has only one components, $\sigma_{x}\left(\sigma_{y}=\right.$ $\left.\sigma_{z}=\sigma_{x y}=\sigma_{y z}=\sigma_{x z}=0\right)$ so that the stress in the local $\left\{\mathrm{x}^{\prime}, \mathrm{y}^{\prime}, \mathrm{z}^{\prime}\right\}$ coordinate system is obtained by the transformation of Eq (4.3):

$$
[\sigma]=\left[\begin{array}{c}
\sigma_{x \prime} \\
\sigma_{y \prime} \\
\sigma_{z \prime} \\
\sigma_{x y \prime} \\
\sigma_{y z \prime} \\
\sigma_{x z \prime}
\end{array}\right]=\left[\begin{array}{c}
\alpha_{1}{ }^{2} \sigma_{x} \\
\beta_{1}{ }^{2} \sigma_{x} \\
\gamma_{1}{ }^{2} \sigma_{x} \\
\beta_{1} \gamma_{1} \sigma_{x} \\
\gamma_{1} \alpha_{1} \sigma_{x} \\
\alpha_{1} \beta_{1} \sigma_{x}
\end{array}\right]
$$

Then since direction cosines between the two coordinate systems are calculated as: $\alpha_{1}=$ $\frac{h}{\sqrt{h^{2}+k^{2}+l^{2}}}, \beta_{1}=\frac{k}{\sqrt{h^{2}+k^{2}+l^{2}}}, \gamma_{1}=\frac{l}{\sqrt{h^{2}+k^{2}+l^{2}}}$, finally Eq. (4.6) can be expressed as

$$
\left[\sigma^{\prime}\right]=\left[\begin{array}{c}
\frac{h^{2}}{h^{2}+k^{2}+l^{2}} \sigma_{x} \\
\frac{k^{2}}{h^{2}+k^{2}+l^{2}} \sigma_{x} \\
\frac{l^{2}}{h^{2}+k^{2}+l^{2}} \sigma_{x} \\
\frac{k l}{h^{2}+k^{2}+l^{2}} \sigma_{x} \\
\frac{u w}{h^{2}+k^{2}+l^{2}} \sigma_{x} \\
\frac{h k}{h^{2}+k^{2}+l^{2}} \sigma_{x}
\end{array}\right]
$$

Thus Eq (4.7) can be used to resolve the uniaxial tensile stress in any direction with respect 
to the local coordinate system for single crystallites in Haynes 282.

\subsubsection{Anisotropic yield stress ratios}

The Hill potential function can be used to describe anisotropic yielding of single grains in Haynes 282, which takes the form [62]:

$$
\mathrm{f}(\sigma)=\sqrt{F\left(\sigma_{22}-\sigma_{33}\right)^{2}+G\left(\sigma_{33}-\sigma_{11}\right)^{2}+H\left(\sigma_{11}-\sigma_{22}\right)^{2}+2 L{\sigma_{23}}^{2}+2 M{\sigma_{31}}^{2}+2 N{\sigma_{12}}^{2}}
$$

where $F, G, H, L, M, N$ are material constants that can be determined via tensile tests of the material in different directions. In $\mathrm{Eq}(4.8), \sigma_{\mathrm{ij}}$ are the stress components in the local crystallographic system, i.e., $\left[\sigma^{\prime}\right]$ through transformation from uniaxial test coordinate to the $3 \mathrm{D}$ coordinate system. To facilitate the evaluation, these constants are often expressed in terms of the yield stress ratio, $R_{i j}$, as

$$
\begin{gathered}
F=\frac{\left(\sigma^{0}\right)^{2}}{2}\left(\frac{1}{\bar{\sigma}_{22}^{2}}+\frac{1}{\bar{\sigma}_{33}^{2}}-\frac{1}{\bar{\sigma}_{11}^{2}}\right)=\frac{1}{2}\left(\frac{1}{R_{22}{ }^{2}}+\frac{1}{R_{33}{ }^{2}}-\frac{1}{R_{11}{ }^{2}}\right) \\
G=\frac{\left(\sigma^{0}\right)^{2}}{2}\left(\frac{1}{\bar{\sigma}_{22}^{2}}+\frac{1}{\bar{\sigma}_{33}^{2}}-\frac{1}{\bar{\sigma}_{11}^{2}}\right)=\frac{1}{2}\left(\frac{1}{R_{33}{ }^{2}}+\frac{1}{R_{11}{ }^{2}}-\frac{1}{R_{22}{ }^{2}}\right) \\
H=\frac{\left(\sigma^{0}\right)^{2}}{2}\left(\frac{1}{\bar{\sigma}_{11}^{2}}+\frac{1}{\bar{\sigma}_{22}^{2}}-\frac{1}{\bar{\sigma}_{33}^{2}}\right)=\frac{1}{2}\left(\frac{1}{R_{11}{ }^{2}}+\frac{1}{R_{22}{ }^{2}}-\frac{1}{R_{33}{ }^{2}}\right) \\
L=\frac{3}{2}\left(\frac{\tau^{0}}{\bar{\sigma}_{23}}\right)^{2}=\frac{3}{2 R_{23}{ }^{2}} \\
M=\frac{3}{2}\left(\frac{\tau^{0}}{\bar{\sigma}_{13}}\right)^{2}=\frac{3}{2 R_{13}{ }^{2}} \\
N=\frac{3}{2}\left(\frac{\tau^{0}}{\bar{\sigma}_{12}}\right)^{2}=\frac{3}{2 R_{12}{ }^{2}}
\end{gathered}
$$

where every $\bar{\sigma}_{i j}$ is the measured yield stress when $\bar{\sigma}_{i j}$ is applied as the only nonzero stress 
component; $\sigma^{o}$ is the reference yield stress; and $\tau^{0}=\frac{\sigma^{0}}{\sqrt{3}}$.

Considering the symmetry of a cubic FCC crystal structure of nickel based single crystal, it can be ascertained that: $\bar{\sigma}_{11}=\bar{\sigma}_{22}=\bar{\sigma}_{33}$ and $\bar{\sigma}_{12}=\bar{\sigma}_{23}=\bar{\sigma}_{13}$ such that $F=G=H$ and $L=M=N$. Hence, the anisotropic yield stress ratios also follow the rule of symmetry: $R_{11}=R_{22}=R_{33}$ and $R_{23}=R_{13}=R_{12}$. Consequently, uniaxial tensile tests in two directions are enough to generate material yielding data for solving Eq (4.8) and obtain the anisotropic yield stress ratios $\left(R_{11}, R_{22} R_{33}, R_{23}, R_{13}, R_{12}\right)$. Since the yield stress ratio $R_{i j}$ are the properties of the crystalline structure, not the absolute magnitude of yield stress, other Ni-base single crystal data, e.g., PWA 1480, can be used to determine the values of $R_{i j}$ for individual grains in Haynes 282. The tensile yield stress data of PWA 1480 in three

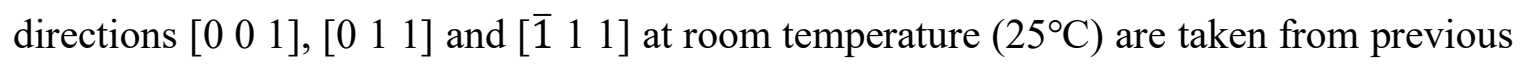
research [108].

Table 4. 1 The yielding strength of PWA 1480 in three directions at room temperature [108]

\begin{tabular}{|c|c|c|c|}
\hline Direction & {$\left[\begin{array}{lll}0 & 0 & 1\end{array}\right]$} & {$\left[\begin{array}{lll}0 & 1 & 1\end{array}\right]$} & {$\left[\begin{array}{lll}\overline{1} & 1 & 1\end{array}\right]$} \\
\hline $0.2 \%$ yielding $(\mathrm{MPa})$ & 1156 & 1020 & 1097 \\
\hline
\end{tabular}

The stress components in the local cubic coordinate system are calculated using Eq (4.7). The reference yield stress is chosen to be $1156 \mathrm{MPa}$ (Table 4.1). By substituting yield strength in any two directions in Table 4.1 to Eq (4.8), in total 3 sets of $R_{i j} \mathrm{~s}$ can be obtained 
from mathematical calculation. The results of calculated stress components $\sigma_{i j}$ for every yielding stress are reported in Table 4.2 while anisotropic yielding stress ratios $R_{i j}$ along with material constants $F=G=H$ and $L=M=N$ are presented in Table 4.3.

Table 4.2 Calculated stress components referring to local coordinate

\begin{tabular}{|c|c|c|c|c|c|c|}
\hline Direction & $\sigma_{11}(\mathrm{MPa})$ & $\sigma_{22}(\mathrm{MPa})$ & $\sigma_{33}(\mathrm{MPa})$ & $\sigma_{12}(\mathrm{MPa})$ & $\sigma_{23}(\mathrm{MPa})$ & $\sigma_{13}(\mathrm{MPa})$ \\
\hline$\left[\begin{array}{lll}0 & 0 & 1\end{array}\right]$ & 0 & 0 & 1156 & 0 & 0 & 0 \\
\hline$\left[\begin{array}{lll}0 & 1 & 1\end{array}\right]$ & 0 & 510 & 510 & 0 & 0 & 510 \\
\hline$\left[\begin{array}{lll}1 & 1 & 1\end{array}\right]$ & 365.67 & 365.67 & 365.67 & -365.67 & -365.67 & 365.67 \\
\hline
\end{tabular}

Table 4.3 Calculated yielding stress ratios and material constants by Hill potential function

\begin{tabular}{|c|c|c|c|c|c|c|}
\hline $\begin{array}{c}\text { Loading } \\
\text { Direction 1 }\end{array}$ & $\begin{array}{c}\text { Loading } \\
\text { Direction 2 }\end{array}$ & $\begin{array}{c}\sigma^{0} \\
(\mathrm{MPa})\end{array}$ & $\mathrm{R}_{11}$ & $\mathrm{R}_{12}$ & $\mathrm{~F}, \mathrm{G}, \mathrm{H}$ & $\mathrm{L}, \mathrm{M}, \mathrm{N}$ \\
\hline$\left[\begin{array}{lll}0 & 0 & 1\end{array}\right]$ & {$\left[\begin{array}{lll}0 & 1 & 1\end{array}\right]$} & 1156 & 1 & 0.8515 & 0.5 & 2.0689 \\
\hline$\left[\begin{array}{lll}0 & 0 & 1\end{array}\right]$ & {$\left[\begin{array}{lll}\overline{1} & 1 & 1\end{array}\right]$} & 1156 & 1 & 0.949 & 0.5 & 1.6657 \\
\hline$\left[\begin{array}{lll}0 & 1 & 1\end{array}\right]$ & {$\left[\begin{array}{lll}\overline{1} & 1 & 1\end{array}\right]$} & 1156 & 0.672 & 0.949 & 0.9032 & 1.6657 \\
\hline
\end{tabular}

As shown in Table 4.3, the calculated yielding stress ratios and material constants vary with selections of loading directions. Since only one set of yielding stress ratios is required to be the input of the FEA model using Abaqus package, the three sets of calculated material constants $F, G, H$ and $L, M, N$ are averaged respectively: $F=G=H=0.6344$, $L=M=N=1.8$. From $\mathrm{Eq}(4.9)$ to $\mathrm{Eq}(4.14)$, the final yielding stress ratios are determined 


$$
\begin{aligned}
& R_{11}=R_{22}=R_{33}=0.8878 \\
& R_{23}=R_{13}=R_{12}=0.9128
\end{aligned}
$$

\subsubsection{Hysteresis behavior}

\subsubsection{Kinematic hardening parameters for LCF simulation}

When the deformation of cyclically loaded material enters plastic regime, it may exhibit strain hardening. An appropriate hardening law is required to describe the progression of yield surface for the material during fatigue. In the Abaqus package, there are three hardening models available to simulate the behavior of metals that are subjected to cyclic loading: isotropic hardening, kinematic hardening and combined isotropic/kinematic hardening. Generally, the combined hardening model provides more accurate description of the stress-strain relation in the plastic regime. Unfortunately, the Abaqus/Standard used in the present study does not provide combined hardening and multilinear kinematic hardening in conjunction with the Hill yield surface (it does for isotropic materials though), therefore linear kinematic hardening is considered to represent the uniaxial behavior in the plastic regime. The reference yield stress and linear kinematic hardening properties will be determined by calibration to the macroscopic stress-strain behavior of Haynes 282 .

To perform elastoplastic analysis, the Abaqus package requires input of the material reference yield stress and linear kinematic hardening rule. These parameters govern the 
material behavior at the microstructural level, but the overall response of the RVE has to match the material coupon behavior. Therefore, at first, an iterative calibration has to be performed with the reference yield stress and linear hardening properties given in Table 4.4 for LCF simulation. The simulation results are compared with experimental hysteresis loops in Figure 4.12 to Figure 4.15. It can be seen that the simulated loops match with the experimental loops reasonably well for $\Delta \varepsilon=0.84 \%, \Delta \varepsilon=0.93 \%$ and $\Delta \varepsilon=1.34 \%$ in both strain and stress ranges. The simulated loop for $\Delta \varepsilon=1.70 \%$ has a higher stress range than the experimental one. This is because the linear kinematic hardening model does have limitations in describing the large strain behavior, and multilinear or other nonlinear hardening is not provided by Abaqus package to use in conjunction with Hill's surface. The significance of this simulation is that by modeling the behavior of each grain with anisotropic elasticity, Hill's yielding and linear kinematic hardening, the overall cyclic behavior of Haynes 282 can be described for its overall elastic modulus, yield stress and hardening behavior. Subsequently, the strain range of $0.84 \%$ and $0.93 \%$ are considered for LCF simulation.

Table 4. 4 Strain hardening parameters for LCF of Haynes 282

\begin{tabular}{|c|c|}
\hline Stress $\sigma(\mathrm{MPa})$ & Plastic strain $\varepsilon_{p}$ \\
\hline 670 & 0.0 \\
\hline 950 & 0.0025 \\
\hline
\end{tabular}




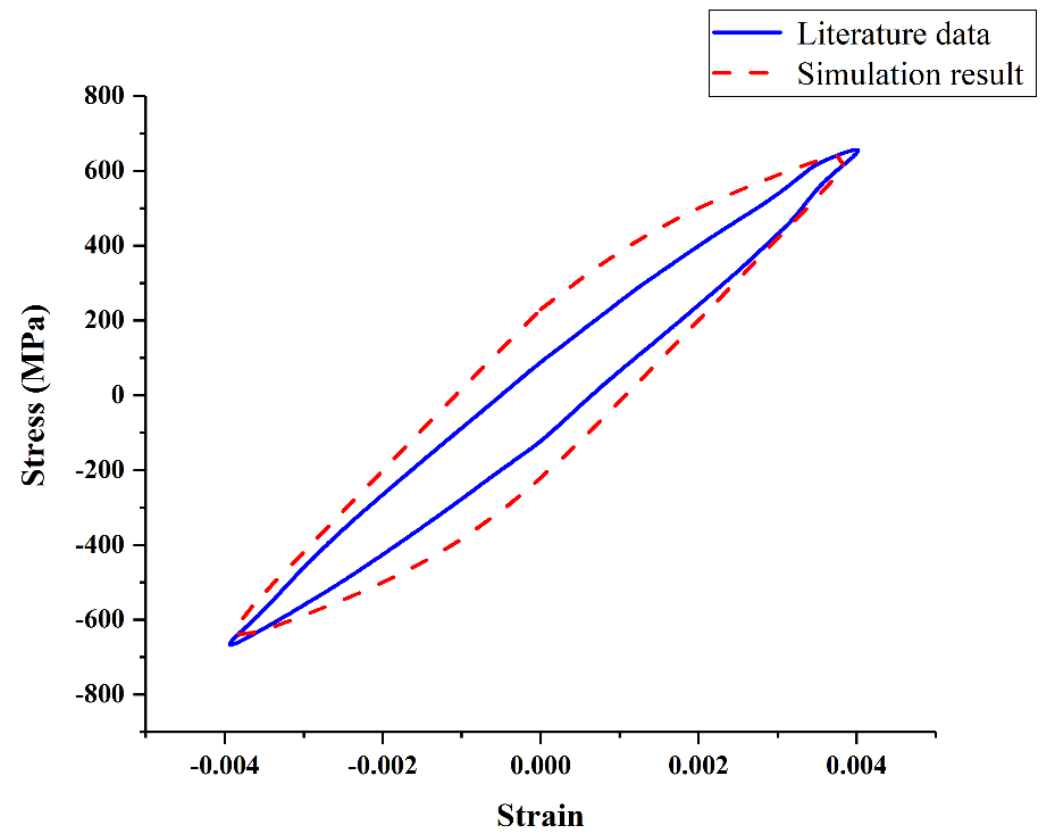

Figure 4. 12 Stabilized hysteresis loops of Haynes 282 at strain range of $0.84 \%$.

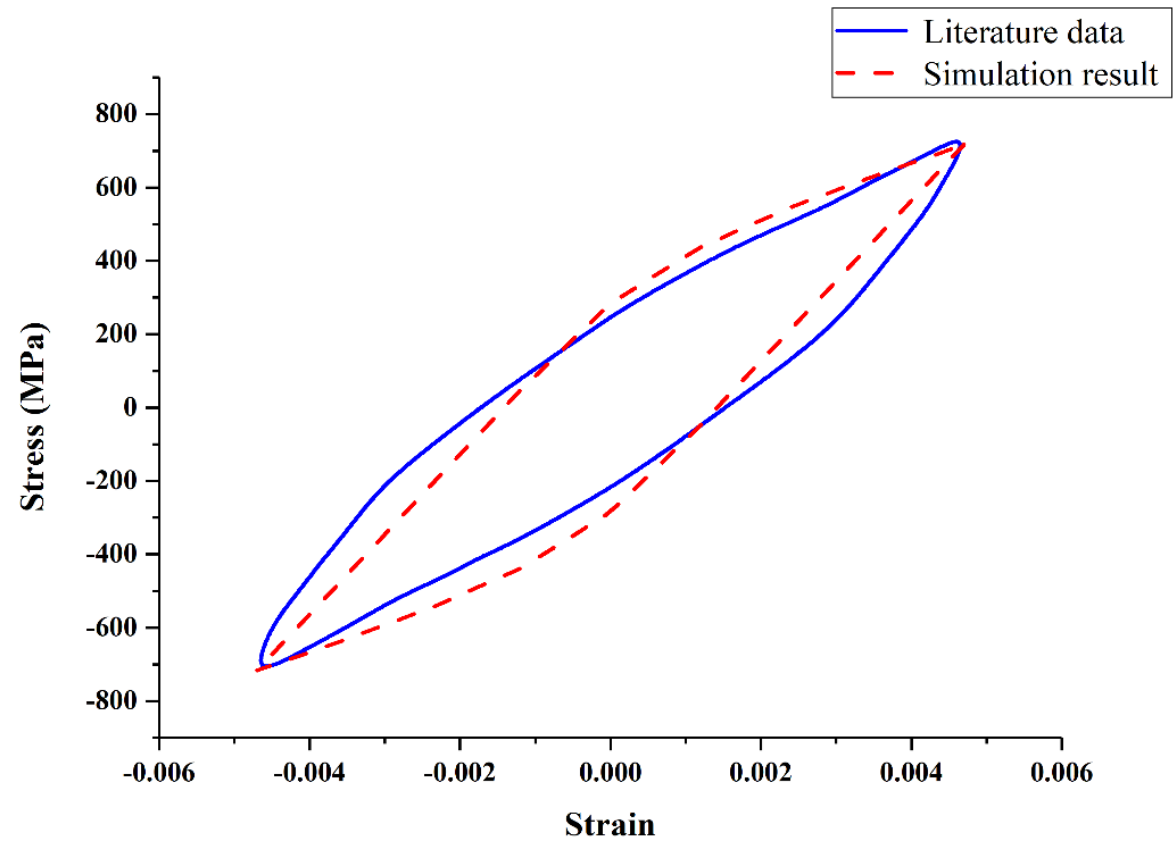

Figure 4. 13 Stabilized hysteresis loops of Haynes 282 at strain range of $0.93 \%$. 


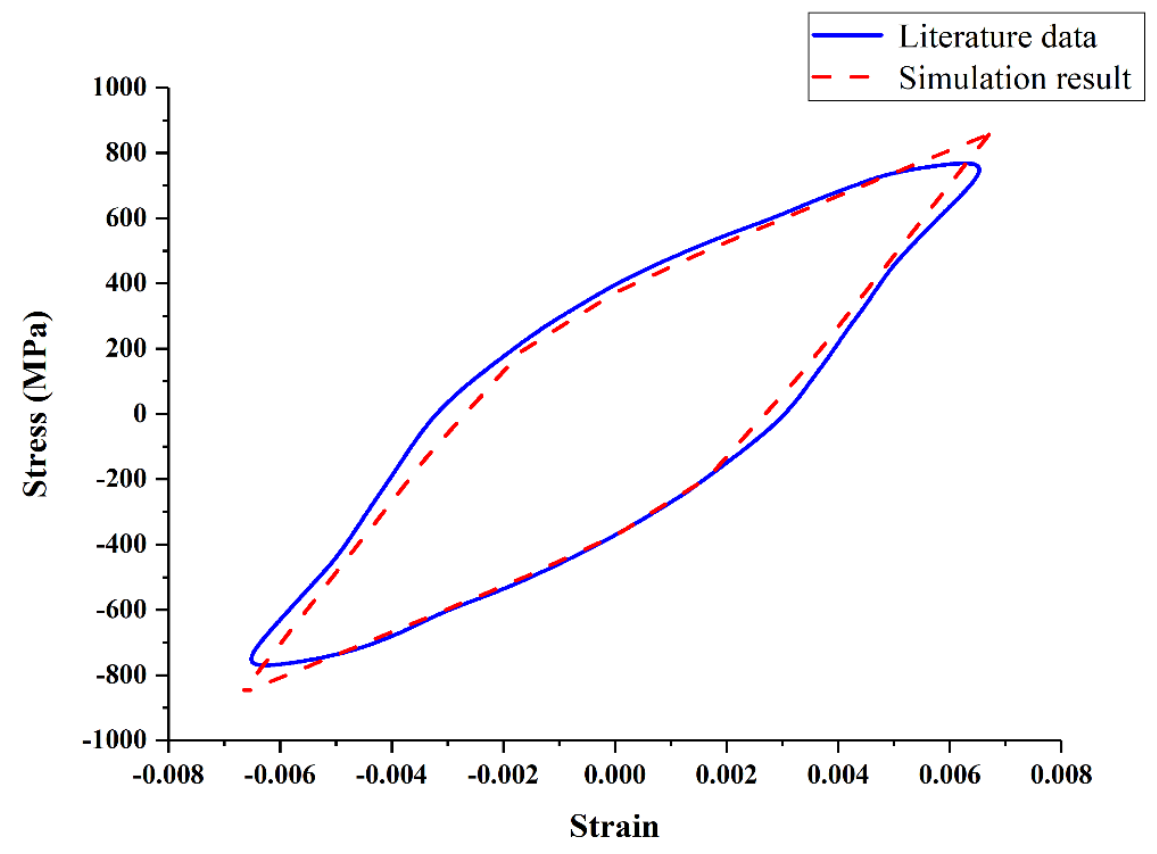

Figure 4. 14 Stabilized hysteresis loops of Haynes 282 at strain range of 1.34\%.

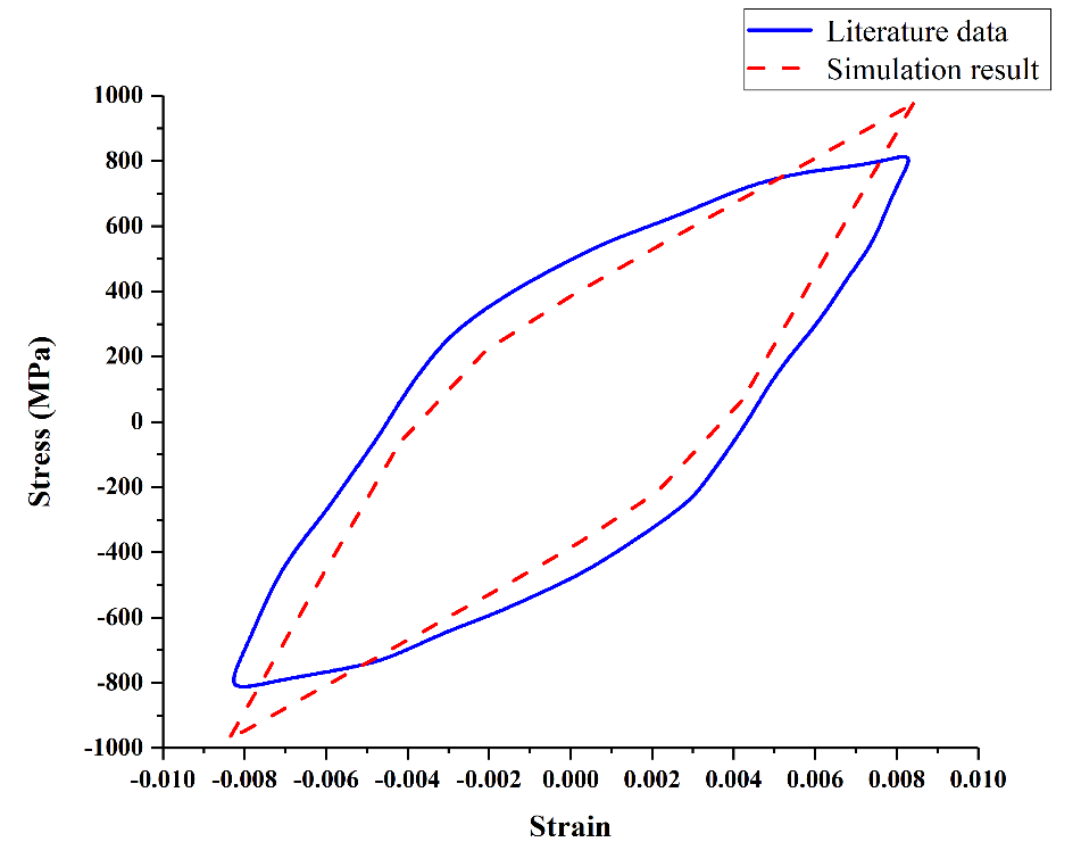

Figure 4. 15 Stabilized hysteresis loops of Haynes 282 at strain range of $1.70 \%$ 


\subsubsection{Kinematic hardening parameters for HCF simulation}

For the HCF simulation, since no hysteresis loops for Haynes 282 are available in literature, yield and strain hardening parameters are determined based on the tensile stress-strain curve from the studies by Ugodilinwa [79], because the material used in this study was in the same heat treatment condition (solution treatment + standard aging) as that used by M. Yang et al. [86]. The calibrated parameters are presented in Table 4.5 and the effectiveness of calibration is verified in Figure 4.16, which shows a good agreement between the simulated tensile curve and the experimental observation.

Table 4. 5 Strain hardening parameters for HCF of Haynes 282

\begin{tabular}{|c|c|}
\hline Stress $\sigma(\mathrm{MPa})$ & Plastic strain $\varepsilon_{p}$ \\
\hline 750 & 0.0 \\
\hline 950 & 0.0088 \\
\hline
\end{tabular}

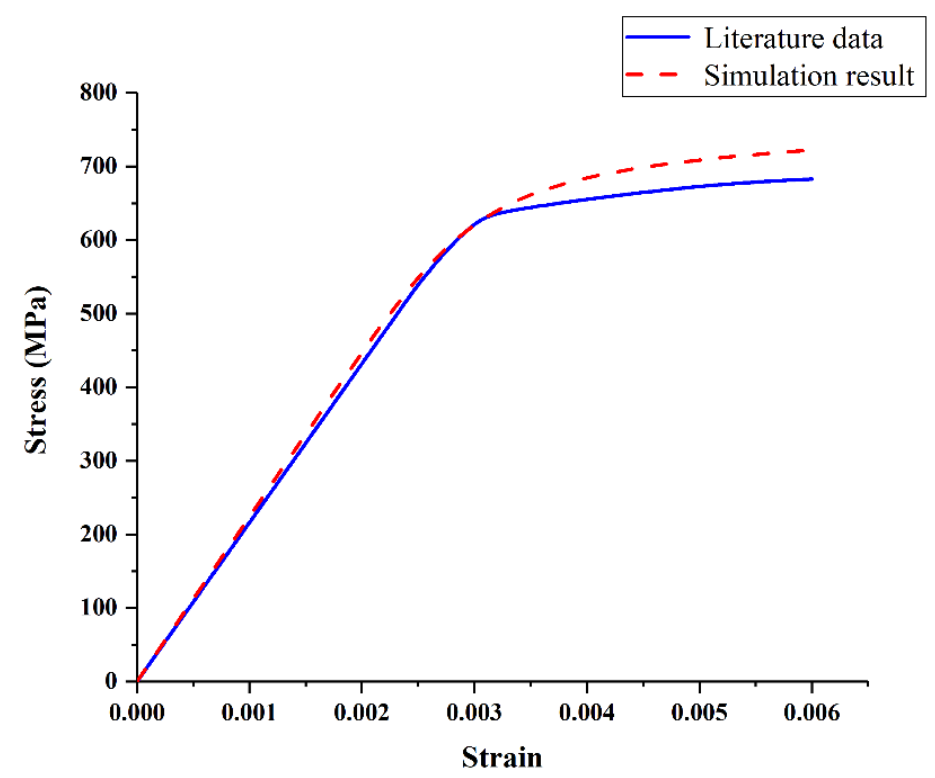

Figure 4. 16 Tensile curves for HCF of Haynes 282. 


\subsection{Fatigue Nucleation Life Prediction of Haynes 282}

\subsubsection{Fatigue at microstructure level}

When an engineering component/structure is under cyclic loading, stress concentrations may be raised by geometrical features of the component/structure to cause cyclic damage associated with local plasticity. This is true at microstructure level, because the Voronoi grain structure represents a significant degree of material heterogeneity. The TMW model allows the prediction of fatigue nucleation life with given material properties and plastic strain range.

In Chapter 3, the strain-based formula Eq (3.1) has shown to be able to predict the macroscopic coupon LCF life for many engineering alloys. In this section Eq (3.1) will be applied at microstructure level to estimate the fatigue nucleation life of Haynes 282. Here "to estimate" means to obtain estimation in the order-of-magnitude sense as the microstructure is randomly generated and the grain orientation is randomly assigned, which are not necessarily true, but the estimation provides a way to assess the possible fatigue scatter due to microstructural effects, analytically.

\subsubsection{Plastic strain range}

For microstructural fatigue analysis using the TMW model, it is important to define the microstructural plastic strain range. In Abaqus the definition of 3D plastic strain magnitude (PEMAG) $\varepsilon^{p}$ is given by 


$$
\varepsilon^{p}=\sqrt{\frac{2}{3} \varepsilon^{p l}: \varepsilon^{p l}}
$$

where $\varepsilon^{p l}$ is a $3 \times 3$ strain tensor that contains all plastic strain components $\varepsilon_{i j}^{p}, \gamma_{i j}^{p}$ :

$$
\varepsilon^{p l}=\left[\begin{array}{ccc}
\varepsilon_{11}^{p} & \frac{1}{2} \gamma_{12}^{p} & \frac{1}{2} \gamma_{13}^{p} \\
\frac{1}{2} \gamma_{21}^{p} & \varepsilon_{22}^{p} & \frac{1}{2} \gamma_{23}^{p} \\
\frac{1}{2} \gamma_{31}^{p} & \frac{1}{2} \gamma_{32}^{p} & \varepsilon_{33}^{p}
\end{array}\right]
$$

Thus, PEMAG can also be expressed as

$$
\varepsilon^{p}=\sqrt{\frac{2}{3}\left[\left(\varepsilon_{11}^{p}\right)^{2}+\left(\varepsilon_{22}^{p}\right)^{2}+\left(\varepsilon_{33}^{p}\right)^{2}+0.5\left(\gamma_{12}^{p}\right)^{2}+0.5\left(\gamma_{13}^{p}\right)^{2}+0.5\left(\gamma_{23}^{p}\right)^{2}\right]}
$$

From Eq (4.19), PEMAG describes the instantaneous magnitude of plastic strain in positive scalar measure. For the RVE under loading in tension, PEMAG reaches the maximum per node when the maximum boundary displacement is applied. Similarly, when the RVE is under compression in the same magnitude, the same (positive) value of PEMAG will be attained. Hence, the total plastic strain range $\Delta \varepsilon_{p}$ experienced by the microstructure through the entire reversed load cycle is

$$
\Delta \varepsilon_{p}=\varepsilon_{t}^{p}+\varepsilon_{c}^{p}
$$

where $\varepsilon_{t}^{p}$ and $\varepsilon_{c}^{p}$ are PEMAG at the maximum strain amplitude during tension and compression. It should be noted that the material would always experience a few cycles of plastic shaken down, which redistributes the stress-strain in the initial microstructure to a plastically deformed microstructure. The shaken-down process is shown in Figure 4.17, where PEMAG is seen to stabilize at a constant level after a few cycles. Thus, the values of $\varepsilon_{t}^{p}$ and $\varepsilon_{c}^{p}$ in stabilized period are used to calculate the plastic strain range $\Delta \varepsilon_{p}$. 
Stabilized PEMAG values for all elements are extracted by using Python Scripts given in Appendix D.

Since crack nucleation preferably occurs at the locations with high localized plastic strain, the element with the maximum value of plastic strain range in the RVE is assumed to be the first crack nucleation site, and the maximum value of PEMAG from Abaqus is input to Eq (3.1) to calculate the fatigue crack nucleation life. The elements on the loaded and constrained planes $(x=500 \mu \mathrm{m})$ and $(x=0 \mu \mathrm{m})$ as well as three unselected free surfaces $(z=500 \mu \mathrm{m}),(y=500 \mu \mathrm{m})$ and $(y=0 \mu \mathrm{m})$ are excluded because of the boundary effect.

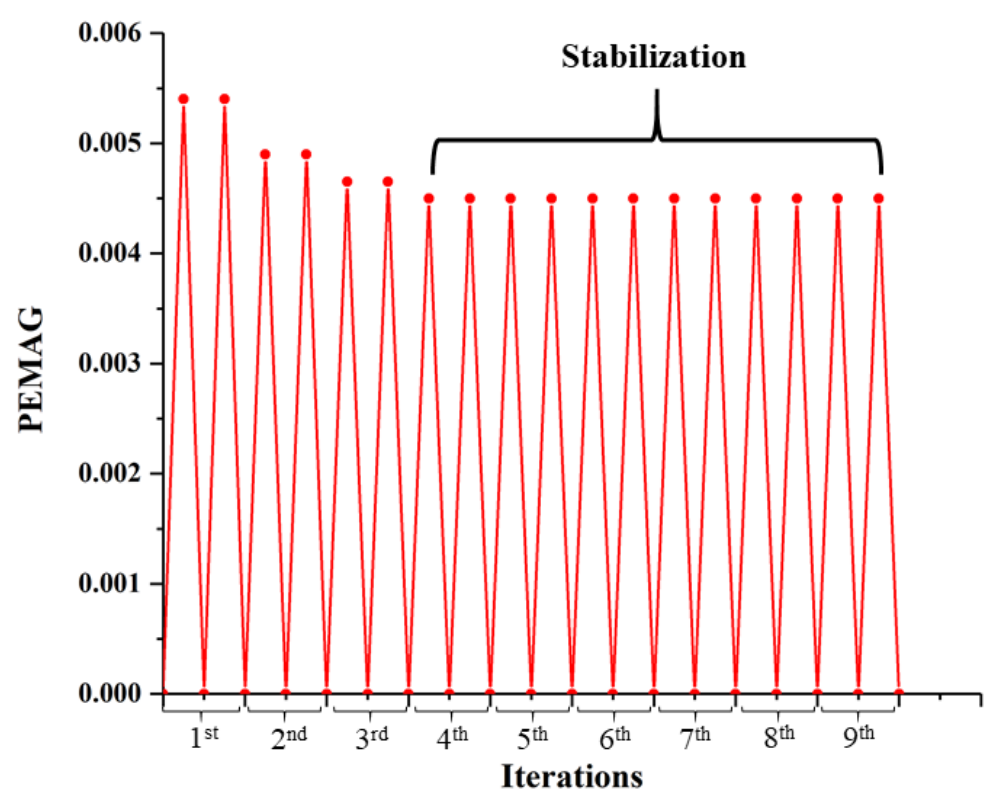

Figure 4. 17 Stabilization of plastic strain magnitude with the number of iteration increased. 


\subsection{Simulation Results and Discussion}

\subsubsection{LCF life prediction}

Twenty (20) LCF simulations are performed for both total strain ranges of $0.84 \%$ and $0.93 \%$, which are denotated as $\operatorname{LCF}_{-} \mathrm{T}_{\mathrm{i}}(\mathrm{i}=1,2,3 \ldots 20)$, to evaluate the localized plastic strain and stress, the fatigue nucleation life and grain orientation effect of Haynes 282.

\subsubsection{Localized plasticity and stress under LCF}

The contour plots of stabilized plastic strain magnitude and Von Mises stress of Haynes 282 at the maximum strain level for LCF- $\mathrm{T}_{14}$ at total strain range of $0.84 \%$ are shown in Figure 4.18 and Figure 4.19, respectively. The heterogeneous plastic strain and stress are localized in different grains in the microstructures, which is a common feature for cyclically loaded polycrystalline metals. The levels of plastic strain concentration and stress concentration vary in different grains, which is due to grain orientation or grain morphology. In addition, it can be observed that the zones of high plasticity are always consistent with that of high localized stress, which well reflects the positive correlation between stress concentration and plastic deformation. Furthermore, large plasticity area is found to preferably occur right on the free surface of the RVE (plane $z=0$ ) or areas close to the surface, which also shows a good agreement with the fact that fatigue crack is prone to nucleate at free surface of a material due to low constraints (The surface roughness cannot be reflected in the FEM simulation but its effect is accounted in Eq (3.1)). The 
distribution of localized plastic flow at free surface is shown in Figure 4.20 where four regions of high plastic strain magnitude are marked with red loops (region 1, 2, 3 and 4) while two regions (region 5 and 6) of low plastic strain magnitude are marked with yellow loops. It has been verified in Figure 4.21 that the large plastic regions are mostly located at the grain boundaries in the microstructure, which are typical geometric discontinuities that induce stress concentration. Moreover, the shape and size of low plastic regions seem to approximately coincide with that of the grains in the microstructure, which can be caused by the effect of particular orientations assigned to these grains.

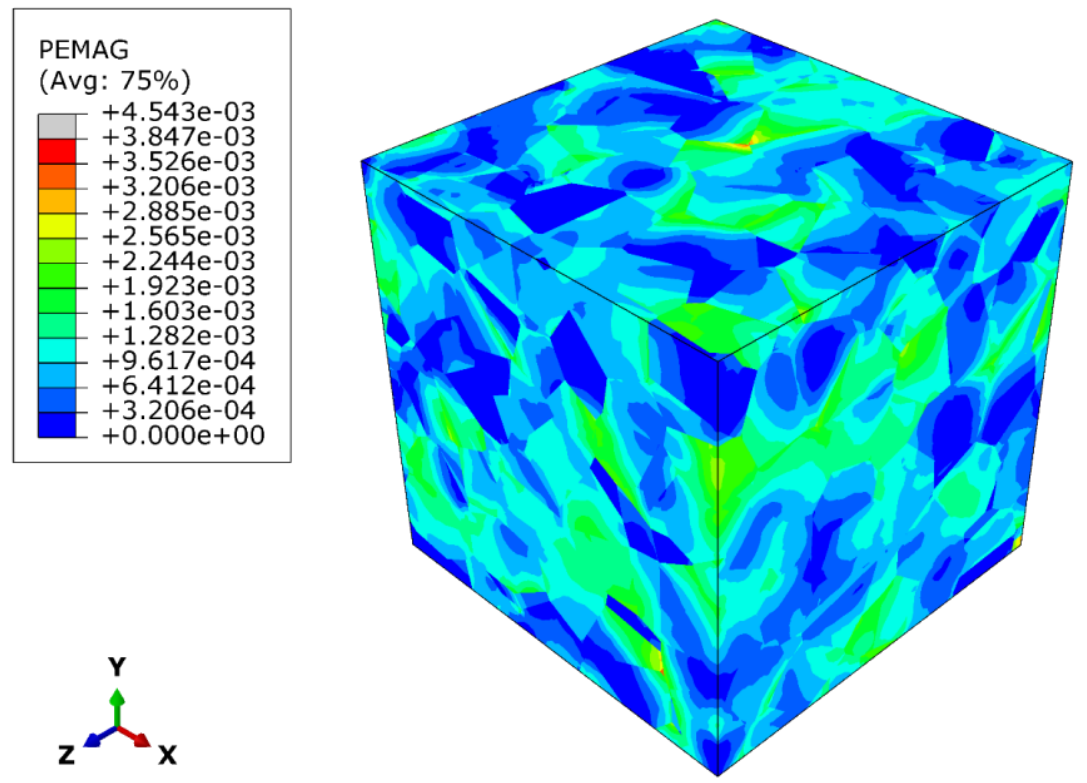

Figure 4. 18 Contour plot of plastic strain magnitude (PEMAG) at the maximum strain level of 4 th cycle for $\mathrm{LCF}_{-\mathrm{T}}{ }_{14}$ (total strain range $\Delta \varepsilon=0.84 \%$ ). 

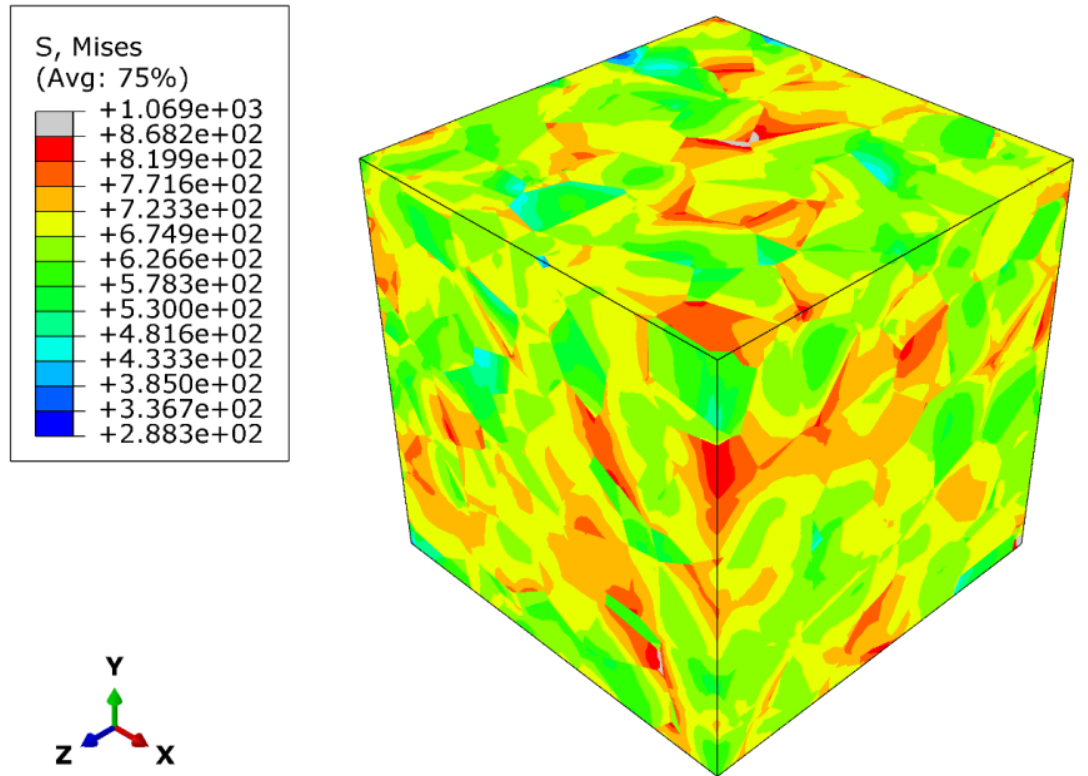

Figure 4. 19 Contour plot of Von Mises stress (S) at the maximum strain level of 4th cycle for LCF-T 14 (total strain range $\Delta \varepsilon=0.84 \%$ ).
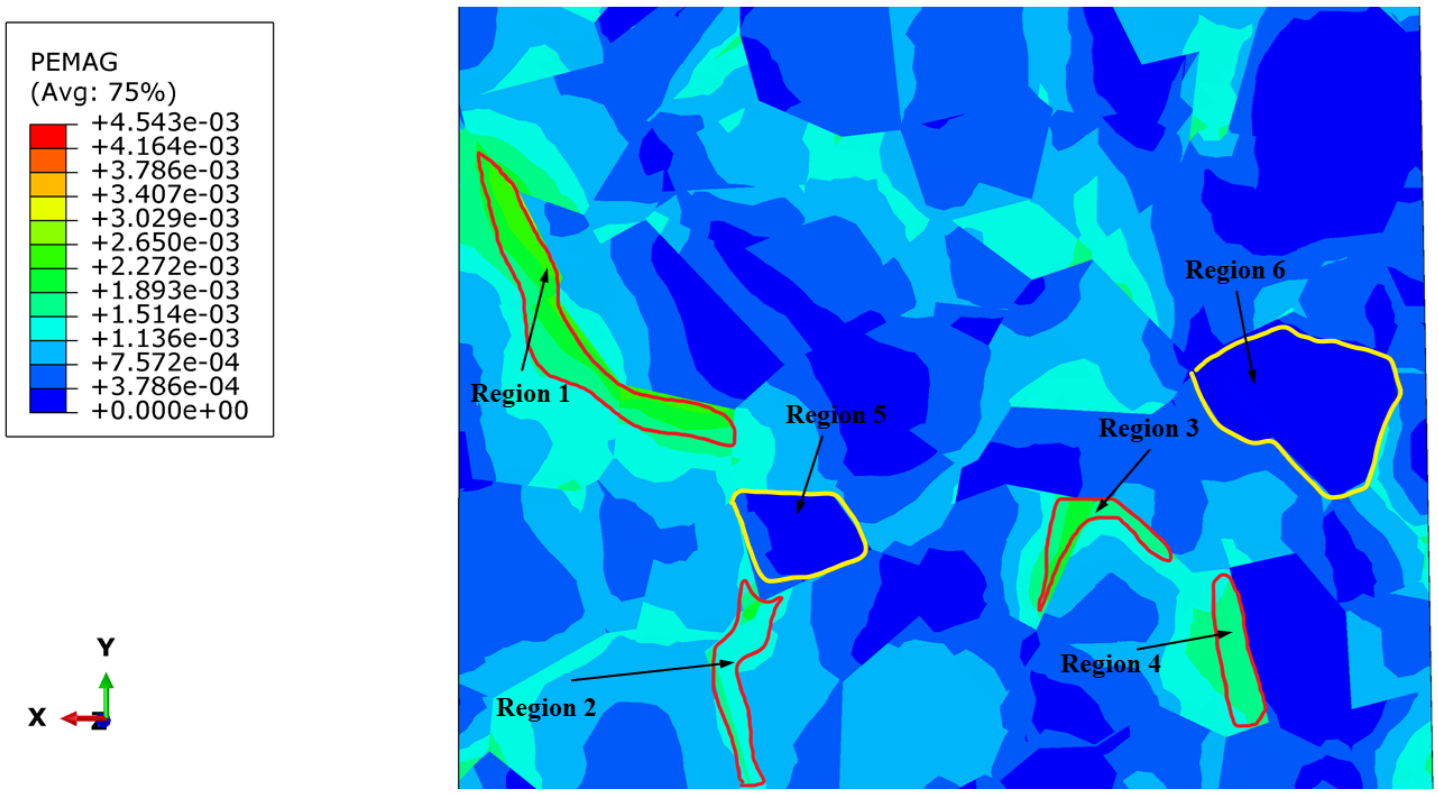

Figure 4. 20 Distribution of localized plastic flow at free surface $(\mathrm{z}=0)$ for LCF-T 14 (total strain range $\Delta \varepsilon=0.84 \%)$. 


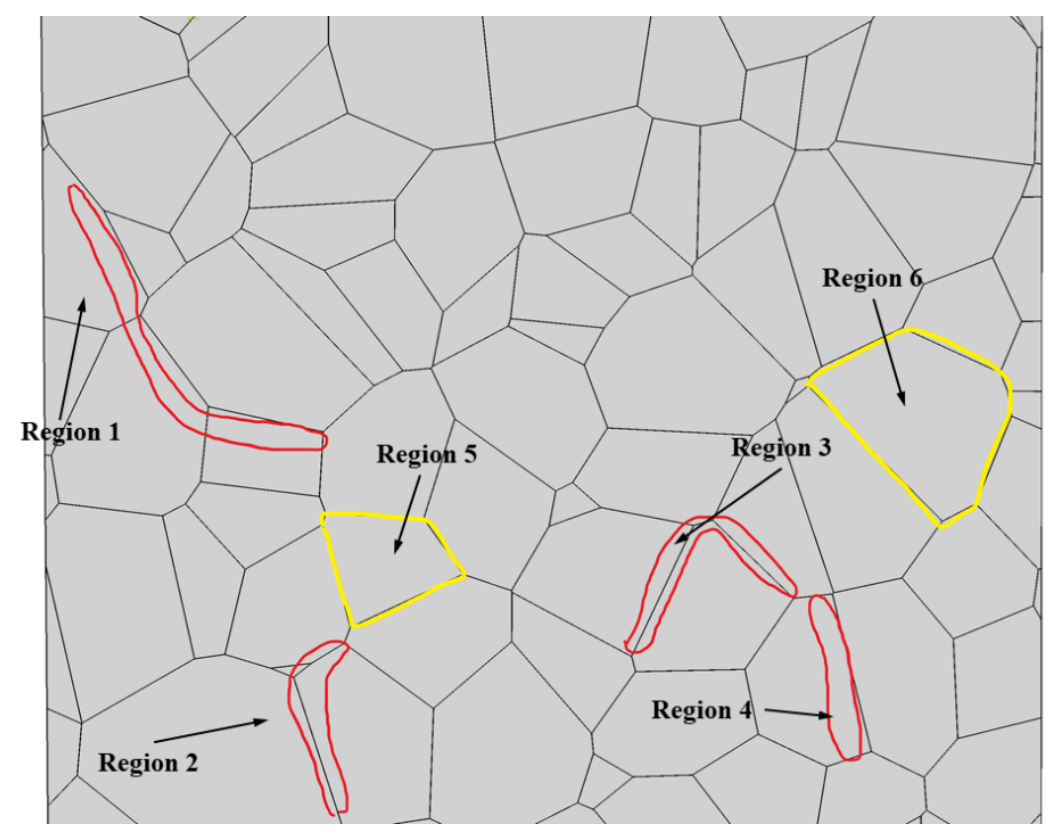

Figure 4. 21 Demonstration of locations of large and small plastic regions.

In the present study, it is assumed that fatigue crack is first nucleated at the place with the largest local plastic strain. Therefore, the first concern in this analysis is to extract the highest value of plastic strain magnitude for each simulation so that the TMW model Eq (3.1) can be used to estimate the fatigue life of Haynes 282. One example is illustrated in this chapter. As shown in Figure 4.22 and Figure 4.23, the highlighted elements have the highest plastic strain magnitude at the maximum strain $\left(\varepsilon_{t}^{p}=0.003315\right)$ under tension and the maximum strain $\left(\varepsilon_{c}^{p}=0.003314\right)$ under compression for LCF-T 6 (strain range $\Delta \varepsilon=$ $0.84 \%)$. Thus, according to Eq (4.21), microscopic plastic strain range $\Delta \varepsilon_{p}=\varepsilon_{t}^{p}+\varepsilon_{c}^{p}=$ $0.003315+0.003314=0.006629$. Then, the maximum plastic strain range $\Delta \varepsilon_{p}$ for all LCF simulations are extracted for the LCF life calculation of Haynes 282. 


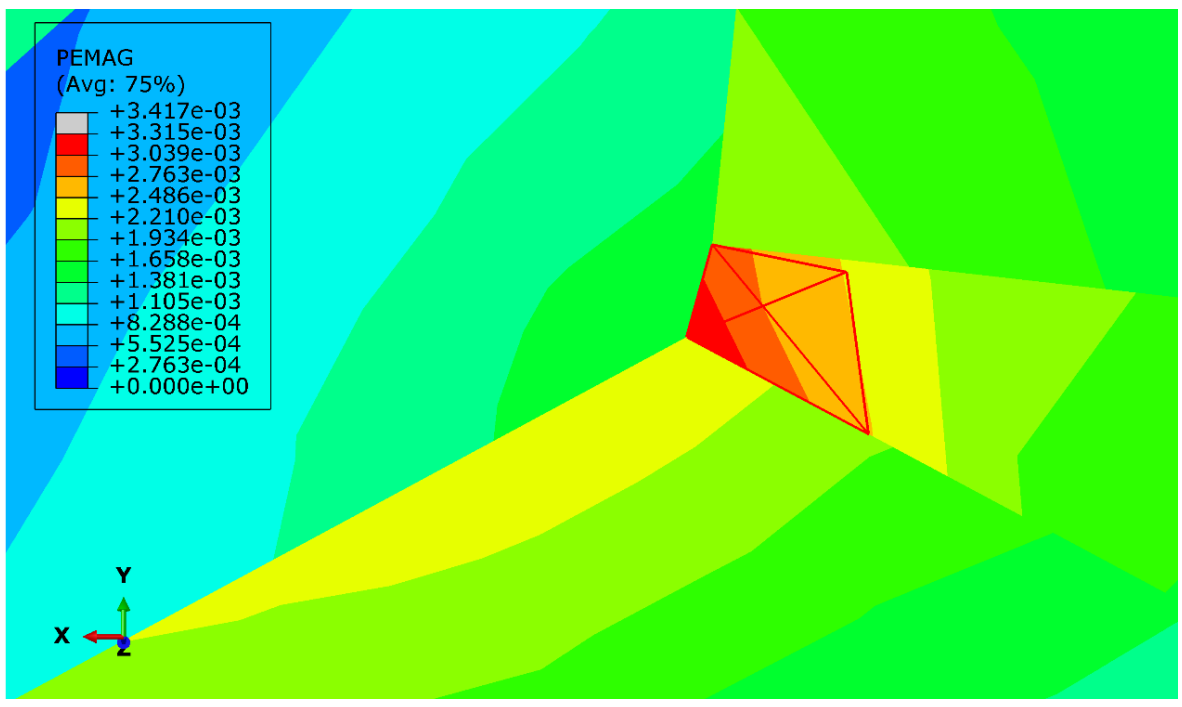

Figure 4. 22 Element with the maximum plastic strain magnitude at the maximum strain of loading for $\mathrm{LCF}_{-} \mathrm{T}_{6}$ (total strain range $\Delta \varepsilon=0.84 \%$ ).

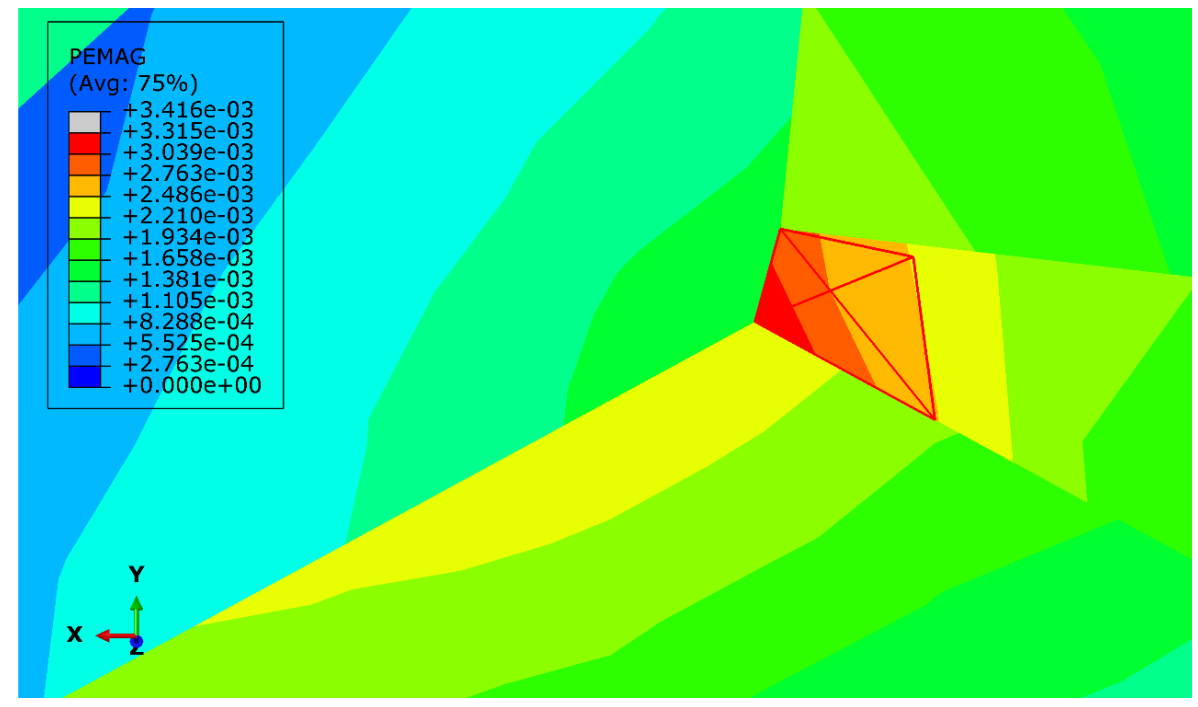

Figure 4. 23 Element with the maximum plastic strain magnitude at the maximum strain of reverse loading for LCF-T 6 (total strain range $\Delta \varepsilon=0.84 \%$ ).

\subsubsection{LCF crack nucleation life}

After the maximum plastic strain range is computed from the FEA simulation, the LCF life of Haynes 282 is estimated using Eq (3.1) with the material properties obtained in section 
3.2.2. The calculated LCF fatigue life of Haynes 282 for all 20 LCF cases along with corresponding maximum plastic strain range $\Delta \varepsilon_{p}$, element number, global plastic strain range and experimental LCF life for total strain range $\Delta \varepsilon=0.84 \%$ and $0.93 \%$ are summarized in Table 4.6 and Table 4.7 respectively. For the strain range of $0.84 \%$, the maximum local plastic strain calculated from the FEA simulation ranges from 0.004953 to 0.006629, which is about five times higher than global plastic strain range reported from previous research [84]. This indicates that the localized plasticity concentration of Haynes 282 is very high at this total strain level. The calculated LCF life varies between 4746.76 to 8501.43 , showing a scattering band of 1.79 , which is reasonably close to the experimental scatter band of 2 for LCF life. In terms of total strain range at $0.93 \%$, similar results are obtained, with maximum plastic strain range spanning from 0.006870 to 0.008968. However, the ratio of microscopic plastic strain range and macroscopic plastic strain range has decreased to approximately 3 times. The scattering of the calculated LCF life for this strain level is ascertained to be around 1.7 , which is slightly smaller than that for $0.84 \%$ strain level. This result conforms to the fact that for the same material, LCF life scattering is mostly smaller than HCF life scattering.

In order to check the validity of predicted LCF life of Haynes 282 based on the FEA and TMW model, the LCF life results are further compared with the experimental data obtained by He et al. [84] in Figure 4.24. The comparison has shown that for both strain ranges, the predictions are in reasonably good agreement with the experimental results, with the error 
rates controlled less than $50 \%$ for the strain range of $0.84 \%$ and about $33.8 \%$ for the strain range of $0.93 \%$. The difference between the predictions and experimental data can be attributed to two reasons. One is that the experimental "LCF life" actually consists of fatigue crack nucleation life and crack propagation life while the predicted microstructural LCF life refers to purely nucleation fraction. Considering the Makkonen's suggestion that crack nucleation consumes $40 \%$ to $90 \%$ of total fatigue life [34], this gap is acceptable. The other could be that the calculated local plastic strain may be overestimated due to the computational error of the FEM software.

Table 4. 6 Calculations of LCF life of Haynes 282 for strain range $\Delta \varepsilon=0.84 \%$

\begin{tabular}{|c|c|c|c|c|c|}
\hline $\begin{array}{c}\text { Simulation } \\
\text { No }\end{array}$ & $\begin{array}{c}\text { Element } \\
\text { No }\end{array}$ & $\begin{array}{l}\text { Maximum local } \\
\text { plastic strain } \\
\text { range }\end{array}$ & $\begin{array}{l}\text { Global } \\
\text { plastic } \\
\text { strain }\end{array}$ & $\begin{array}{l}\text { Calculated } \\
\text { LCF life }\end{array}$ & $\begin{array}{l}\text { Experimental } \\
\text { LCF life }\end{array}$ \\
\hline 1 & 391059 & 0.005273 & \multirow{20}{*}{0.001004} & 7502.02 & \multirow{20}{*}{13414} \\
\hline 2 & 675852 & 0.006032 & & 5732.86 & \\
\hline 3 & 285392 & 0.005886 & & 6020.79 & \\
\hline 4 & 940008 & 0.005055 & & 8163.03 & \\
\hline 5 & 947844 & 0.004943 & & 8536.68 & \\
\hline 6 & 655280 & 0.006629 & & 4746.76 & \\
\hline 7 & 713650 & 0.005068 & & 8121.20 & \\
\hline 8 & 929605 & 0.005675 & & 6475.84 & \\
\hline 9 & 1003581 & 0.005369 & & 7236.14 & \\
\hline 10 & 668319 & 0.005347 & & 7295.81 & \\
\hline 11 & 711169 & 0.005872 & & 6049.53 & \\
\hline 12 & 911846 & 0.006089 & & 5626.03 & \\
\hline 13 & 280279 & 0.004953 & & 8501.43 & \\
\hline 14 & 871498 & 0.006009 & & 5776.83 & \\
\hline 15 & 930491 & 0.005701 & & 6417.88 & \\
\hline 16 & 765889 & 0.005551 & & 6769.42 & \\
\hline 17 & 826302 & 0.005721 & & 6373.09 & \\
\hline 18 & 902989 & 0.005978 & & 5836.89 & \\
\hline 19 & 97132 & 0.005313 & & 7389.48 & \\
\hline 20 & 450313 & 0.005459 & & 6999.46 & \\
\hline
\end{tabular}


Table 4. 7 Calculations of LCF life of Haynes 282 for strain range $\Delta \varepsilon=0.93 \%$

\begin{tabular}{|c|c|c|c|c|c|}
\hline $\begin{array}{l}\text { Simulation } \\
\text { No }\end{array}$ & $\begin{array}{l}\text { Element } \\
\text { No }\end{array}$ & $\begin{array}{l}\text { Maximum local } \\
\text { plastic strain }\end{array}$ & $\begin{array}{l}\text { Global } \\
\text { plastic } \\
\text { strain }\end{array}$ & $\begin{array}{l}\text { Calculated } \\
\text { LCF life }\end{array}$ & $\begin{array}{l}\text { Experimental } \\
\text { LCF life }\end{array}$ \\
\hline 1 & 391059 & 0.007023 & \multirow{20}{*}{0.002892} & 4229.12 & \multirow{20}{*}{5439} \\
\hline 2 & 675852 & 0.008036 & & 3230.08 & \\
\hline 3 & 285392 & 0.007896 & & 3345.64 & \\
\hline 4 & 940008 & 0.007030 & & 4220.69 & \\
\hline 5 & 947844 & 0.006870 & & 4419.57 & \\
\hline 6 & 655280 & 0.008968 & & 2593.60 & \\
\hline 7 & 713650 & 0.007062 & & 4182.52 & \\
\hline 8 & 929605 & 0.007780 & & 3446.15 & \\
\hline 9 & 1003581 & 0.007496 & & 3712.23 & \\
\hline 10 & 668319 & 0.007370 & & 3840.24 & \\
\hline 11 & 711169 & 0.007878 & & 3360.95 & \\
\hline 12 & 911846 & 0.008290 & & 3035.18 & \\
\hline 13 & 280279 & 0.007336 & & 3875.92 & \\
\hline 14 & 871498 & 0.008162 & & 3131.13 & \\
\hline 15 & 930491 & 0.007788 & & 3439.08 & \\
\hline 16 & 765889 & 0.007658 & & 3556.83 & \\
\hline 17 & 826302 & 0.007800 & & 3428.50 & \\
\hline 18 & 902989 & 0.008036 & & 3230.08 & \\
\hline 19 & 97132 & 0.007464 & & 3744.13 & \\
\hline 20 & 450313 & 0.007440 & & 3768.32 & \\
\hline
\end{tabular}




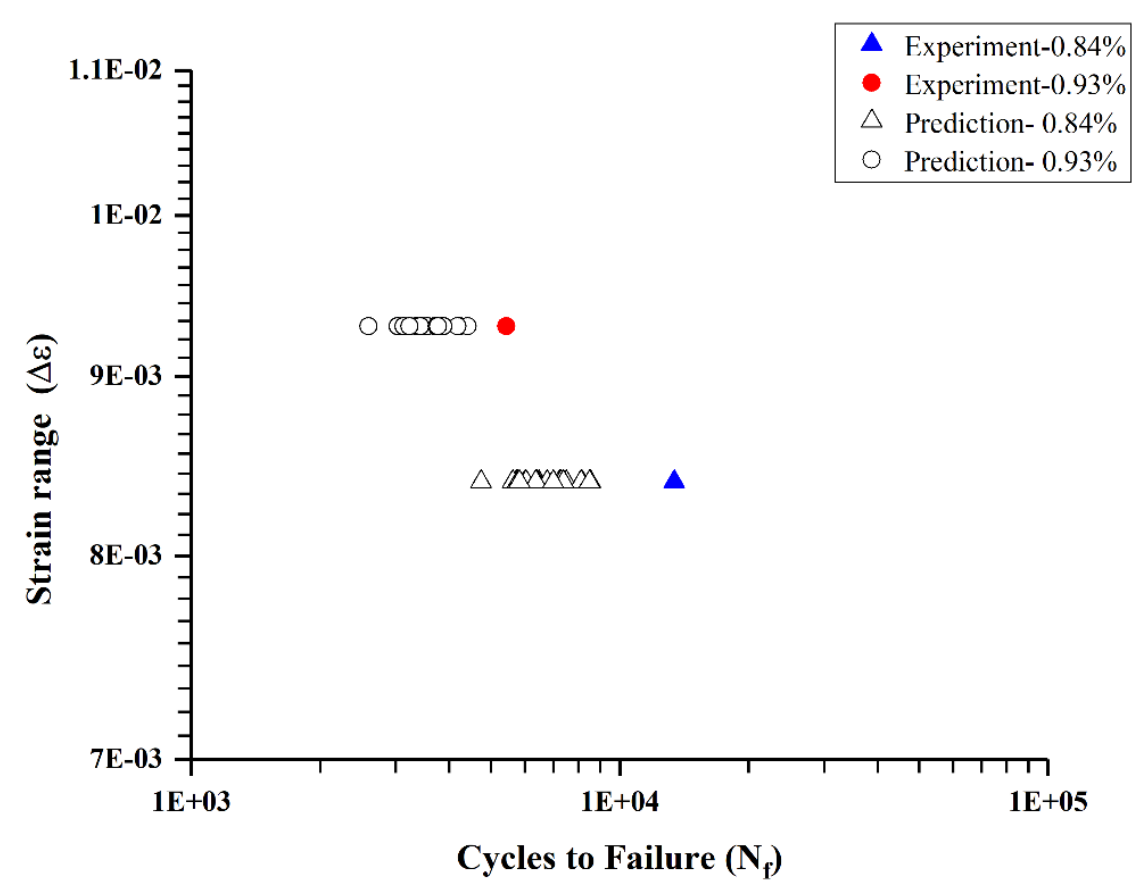

Figure 4. 24 Comparison between calculated LCF crack nucleation life and experimental total-life for stain ranges of $84 \%$ and $93 \%$.

\subsubsection{Grain orientation effect on LCF}

The effect of grain orientation on the LCF life of Haynes 282 is investigated in this section. For each LCF simulation, the grain containing the elements with maximum local plastic strain range and three grains with zero local plastic strain range are selected. The Miller Indices $h, k, l$ and three Bunge Euler angles of orientations for these grains $\varphi, \theta, \psi$ are identified and presented in the inverse pole figure through Matlab coding. According to Figure 4.25 and Figure 4.26, the grains with the maximum plastic strain range are mostly oriented towards octahedral direction $\left[\begin{array}{lll}\overline{1} & 1 & 1\end{array}\right]$ and only three grains locate towards direction [0 $\left.\begin{array}{ll}0 & 1\end{array}\right]$. Figure 4.27 and Figure 4.28 show that the grains with zero plastic strain range tend to locate towards cubic direction $\left[\begin{array}{lll}0 & 0 & 1\end{array}\right]$. This phenomenon can be rationalized by stress 
distribution in "hard" grains and "soft" grains. In FCC materials, [ $\left[\begin{array}{lll}1 & 1 & 1\end{array}\right]$ and $\left[\begin{array}{lll}0 & 1 & 1\end{array}\right]$ grains have high elastic modulus and [0 0 1] grains have low elastic modulus [109]. When strain

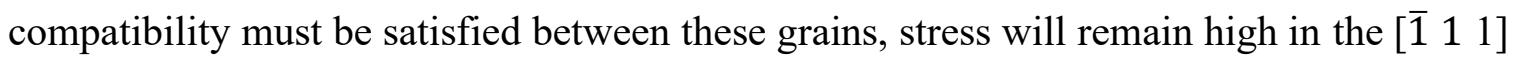
and [ $\left.\begin{array}{lll}0 & 1 & 1\end{array}\right]$ grains, and when the stress goes above the yield, plastic strain must be generated to maintain the continuum strain compatibility. Similar observation on another Ni-base superalloy has been reported [110]. Also, it was found that at room temperature Ni-based single crystal superalloys had much lower yield strength in family of [ $\left[\begin{array}{lll}1 & 1 & 1\end{array}\right]$ than that in family of [l $\left.\begin{array}{lll}0 & 1\end{array}\right]$ [109] [108]. The above argument, the metallurgical as well as mechanical test evidence all support to the simulation prediction revealing that fatigue crack nucleation is more likely to occur in the grains of [ $\left[\begin{array}{lll}1 & 1 & 1\end{array}\right]$ family of Haynes 282.

Moreover, high misalignment of adjacent grains is found to be partially responsible for large plasticity region at grain boundary. Four adjacent grains in one large plasticity region (region 1 marked with red line in Figure 4.21) at grain boundary are selected, as shown in Figure 4.29. The inverse pole figure of selected grains in Figure 4.30 shows that the orientations of these grains depart from each other. 
Table 4. 8 Orientations of grains with maximum plastic strain range for LCF simulations

\begin{tabular}{|c|c|c|c|c|c|c|c|}
\hline Simulation & Grain No. & $\mathrm{h}$ & $\mathrm{k}$ & 1 & $\varphi\left(^{\circ}\right)$ & $\theta\left(^{\circ}\right)$ & $\psi\left({ }^{\circ}\right)$ \\
\hline 1 & 237 & -9 & 9 & 11 & 216.226 & 64.2595 & 212.765 \\
\hline 2 & 338 & -3 & 8 & 9 & 344.617 & 67.1098 & 137.421 \\
\hline 3 & 189 & -8 & 10 & 11 & 283.586 & 142.572 & 323.399 \\
\hline 4 & 433 & -5 & 6 & 7 & 137.138 & 101.385 & 129.91 \\
\hline 5 & 436 & 0 & 5 & 6 & 1.4442 & 118.292 & 40.4199 \\
\hline 6 & 331 & -8 & 10 & 11 & 111.268 & 136.446 & 65.4289 \\
\hline 7 & 350 & -5 & 6 & 9 & 51.898 & 105.863 & 158.001 \\
\hline 8 & 430 & -6 & 7 & 8 & 213.754 & 63.5522 & 212.387 \\
\hline 9 & 465 & -6 & 7 & 8 & 41.9022 & 98.8942 & 146.608 \\
\hline 10 & 335 & -3 & 9 & 10 & 247.683 & 135.644 & 44.152 \\
\hline 11 & 349 & -7 & 8 & 8 & 74.3891 & 142.378 & 29.4358 \\
\hline 12 & 420 & -5 & 5 & 9 & 209.079 & 112.582 & 130.636 \\
\hline 13 & 286 & -7 & 7 & 9 & 312.89 & 112.248 & 21.422 \\
\hline 14 & 407 & -5 & 5 & 6 & 281.827 & 138.516 & 150.019 \\
\hline 15 & 431 & -8 & 9 & 10 & 260.521 & 35.9404 & 62.8624 \\
\hline 16 & 372 & -7 & 8 & 10 & 148.65 & 70.4539 & 63.8928 \\
\hline 17 & 395 & -7 & 10 & 11 & 335.173 & 93.9504 & 220.381 \\
\hline 18 & 291 & -3 & 5 & 6 & 131.985 & 104.081 & 133.185 \\
\hline 19 & 110 & -9 & 10 & 11 & 219.277 & 89.6772 & 318.726 \\
\hline 20 & 256 & -3 & 3 & 5 & 330.647 & 68.7367 & 69.7864 \\
\hline
\end{tabular}


Table 4. 9 Orientations of grains with zero plastic strain range for LCF simulations

\begin{tabular}{|c|c|c|c|c|c|c|c|}
\hline Simulation & Grain No & $\mathrm{h}$ & $\mathrm{k}$ & 1 & $\varphi\left(^{\circ}\right)$ & $\theta\left(^{\circ}\right)$ & $\psi\left({ }^{\circ}\right)$ \\
\hline \multirow{3}{*}{1} & 514 & 0 & 1 & 9 & 2.85785 & 152.045 & 85.9858 \\
\hline & 536 & -1 & 3 & 11 & 319.222 & 156.038 & 327.268 \\
\hline & 318 & -1 & 2 & 9 & 79.8326 & 99.2582 & 107.536 \\
\hline \multirow{3}{*}{2} & 180 & -1 & 2 & 12 & 348.709 & 134.913 & 77.3502 \\
\hline & 388 & -2 & 2 & 11 & 255.584 & 88.3776 & 128.86 \\
\hline & 421 & 0 & 1 & 11 & 2.08501 & 56.2413 & 263.435 \\
\hline \multirow{3}{*}{3} & 183 & 0 & 1 & 6 & 178.946 & 177.453 & 349.825 \\
\hline & 321 & -5 & 8 & 11 & 306.435 & 154.684 & 346.248 \\
\hline & 626 & -4 & 4 & 9 & 213.944 & 44.5357 & 87.6722 \\
\hline \multirow{3}{*}{4} & 268 & -1 & 3 & 12 & 352.027 & 28.2724 & 21.832 \\
\hline & 351 & -3 & 5 & 10 & 153.791 & 87.2943 & 255.259 \\
\hline & 165 & -1 & 1 & 7 & 351.225 & 97.9328 & 260.717 \\
\hline \multirow{3}{*}{5} & 210 & 0 & 1 & 4 & 193.546 & 78.7969 & 85.3147 \\
\hline & 396 & -1 & 2 & 7 & 255.342 & 101.455 & 100.94 \\
\hline & 535 & 0 & 1 & 5 & 177.461 & 139.366 & 76.6755 \\
\hline \multirow{3}{*}{6} & 174 & 0 & 1 & 4 & 357.189 & 54.8677 & 77.7293 \\
\hline & 392 & -2 & 3 & 10 & 333.215 & 27.174 & 97.8306 \\
\hline & 464 & 0 & 6 & 11 & 147.307 & 118.617 & 251.153 \\
\hline \multirow{3}{*}{7} & 293 & -1 & 2 & 12 & 183.671 & 87.3332 & 189.434 \\
\hline & 337 & -2 & 8 & 11 & 237.049 & 74.6231 & 81.1217 \\
\hline & 470 & -1 & 3 & 6 & 82.9385 & 26.9203 & 178.664 \\
\hline
\end{tabular}




\begin{tabular}{|c|c|c|c|c|c|c|c|}
\hline \multirow{3}{*}{8} & 110 & -1 & 1 & 8 & 29.6894 & 163.02 & 201.857 \\
\hline & 332 & -1 & 1 & 10 & 82.9361 & 94.3058 & 181.708 \\
\hline & 479 & 0 & 0 & 1 & 177.913 & 38.2778 & 270.272 \\
\hline \multirow{3}{*}{9} & 189 & -4 & 5 & 11 & 253.756 & 115.064 & 186.286 \\
\hline & 394 & -1 & 2 & 9 & 93.7522 & 102.794 & 310.379 \\
\hline & 553 & 0 & 1 & 9 & 186.655 & 71.636 & 176.856 \\
\hline \multirow{3}{*}{10} & 269 & 0 & 0 & 1 & 269.486 & 92.0158 & 185.108 \\
\hline & 459 & -2 & 5 & 9 & 331.177 & 94.9459 & 74.0576 \\
\hline & 536 & -1 & 2 & 11 & 263.63 & 100.118 & 311.301 \\
\hline \multirow{3}{*}{11} & 167 & -2 & 3 & 10 & 349.092 & 108.247 & 70.4442 \\
\hline & 391 & 0 & 0 & 1 & 170.297 & 10.7257 & 190.589 \\
\hline & 481 & -2 & 5 & 10 & 116.73 & 80.8289 & 220.754 \\
\hline \multirow{3}{*}{12} & 201 & -1 & 1 & 5 & 193.18 & 62.9973 & 342.711 \\
\hline & 385 & 0 & 1 & 12 & 180.994 & 105.568 & 3.35095 \\
\hline & 545 & -2 & 4 & 11 & 297.066 & 22.1553 & 141.388 \\
\hline \multirow{3}{*}{13} & 353 & -2 & 6 & 11 & 226.087 & 41.0949 & 152.854 \\
\hline & 135 & 0 & 1 & 12 & 89.7914 & 85.673 & 338.946 \\
\hline & 526 & 0 & 3 & 10 & 272.162 & 106.724 & 101.684 \\
\hline \multirow{3}{*}{14} & 106 & 0 & 1 & 12 & 175.541 & 129.271 & 359.053 \\
\hline & 7 & 0 & 1 & 3 & 2.41986 & 154.866 & 201.063 \\
\hline & 515 & -2 & 5 & 11 & 189.821 & 114.411 & 69.4479 \\
\hline \multirow[b]{2}{*}{15} & 187 & -1 & 2 & 7 & 73.575 & 83.7134 & 95.4374 \\
\hline & 496 & -1 & 2 & 7 & 310.543 & 159.285 & 231.718 \\
\hline
\end{tabular}




\begin{tabular}{|c|c|c|c|c|c|c|c|}
\hline & 619 & -2 & 5 & 12 & 156.538 & 80.3986 & 264.296 \\
\hline \multirow{3}{*}{16} & 168 & -1 & 4 & 12 & 83.177 & 106.89 & 234.041 \\
\hline & 535 & 0 & 1 & 6 & 2.05123 & 80.8561 & 259.796 \\
\hline & 606 & -2 & 5 & 12 & 156.538 & 80.3986 & 264.296 \\
\hline \multirow{3}{*}{17} & 432 & 0 & 1 & 12 & 350.715 & 26.4377 & 97.1272 \\
\hline & 465 & -1 & 1 & 9 & 189.173 & 141.074 & 90.6354 \\
\hline & 601 & 0 & 1 & 7 & 94.9322 & 96.8264 & 233.295 \\
\hline \multirow{3}{*}{18} & 248 & -2 & 2 & 11 & 87.1252 & 104.129 & 132.211 \\
\hline & 366 & 0 & 2 & 11 & 8.11392 & 16.4991 & 92.123 \\
\hline & 471 & 0 & 1 & 4 & 201.496 & 139.447 & 18.8186 \\
\hline \multirow{3}{*}{19} & 579 & 0 & 1 & 3 & 262.671 & 72.6324 & 205.026 \\
\hline & 402 & 0 & 1 & 12 & 153.195 & 175.85 & 156.381 \\
\hline & 473 & -1 & 4 & 11 & 249.595 & 88.1912 & 340.571 \\
\hline \multirow{3}{*}{20} & 198 & -1 & 2 & 11 & 189.185 & 145.391 & 87.6607 \\
\hline & 324 & -1 & -1 & 2 & 92.9976 & 93.9336 & 278.188 \\
\hline & 338 & 0 & 0 & 1 & 171.676 & 166.416 & 261.246 \\
\hline
\end{tabular}




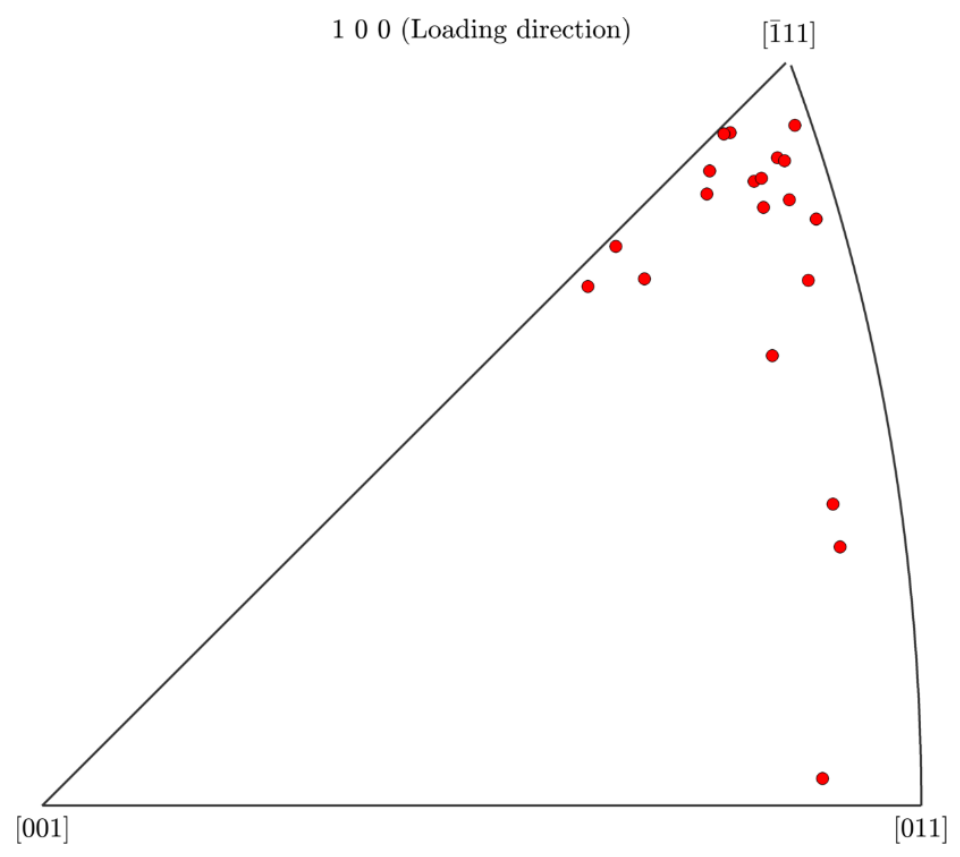

Figure 4. 25 Inverse pole figure of grains with maximum plastic strain range for all LCF simulations.

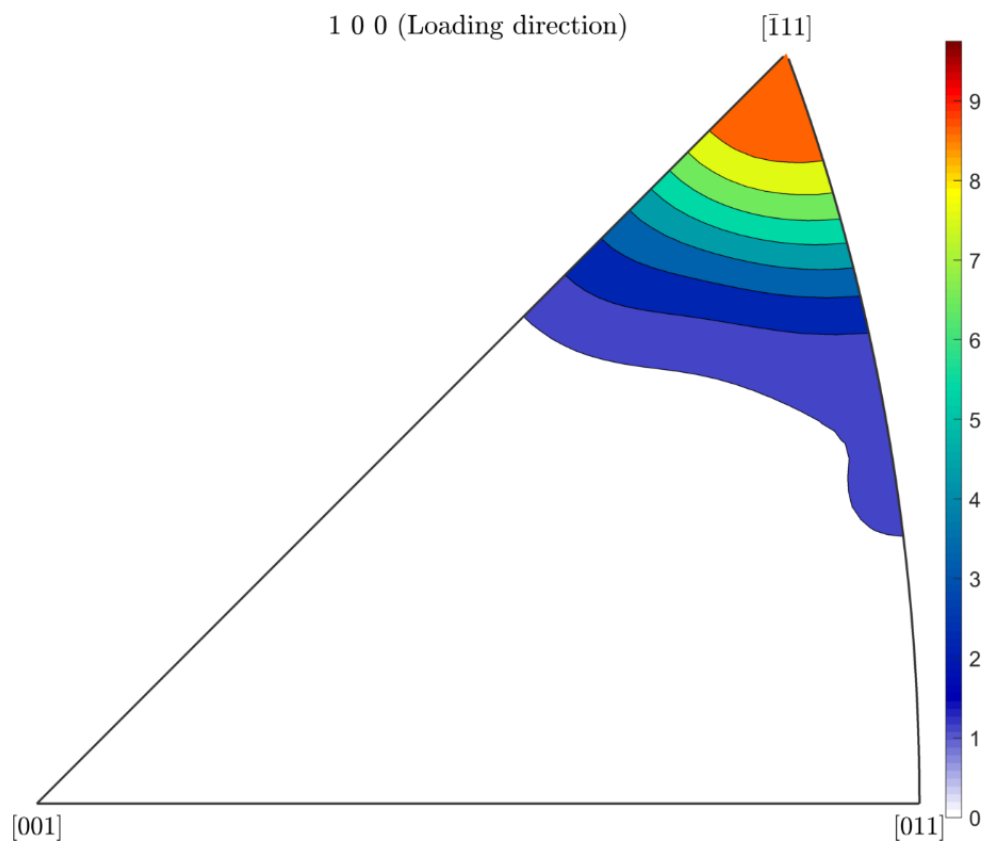

Figure 4. 26 Contour plots of orientations of grains with maximum plastic 


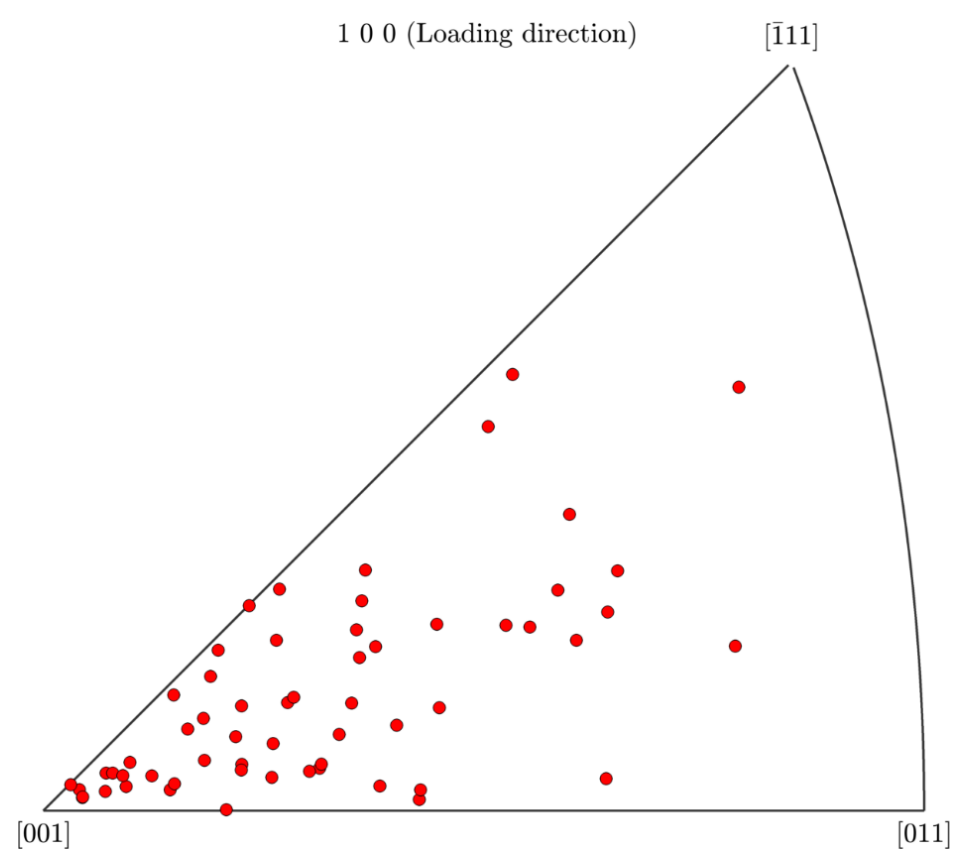

Figure 4. 27 Inverse pole figure for grains with zero plastic strain range for all LCF simulations.

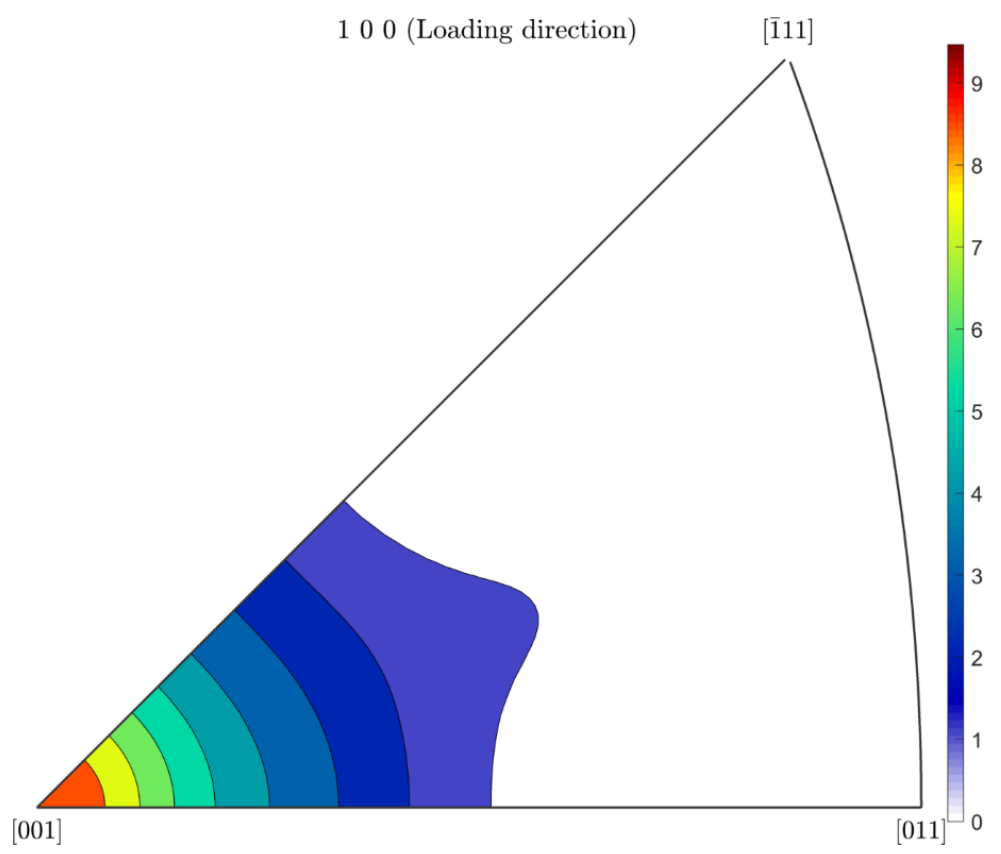

Figure 4. 28 Contour plots of orientations of grains with zero plastic strain range for all LCF simulations. 


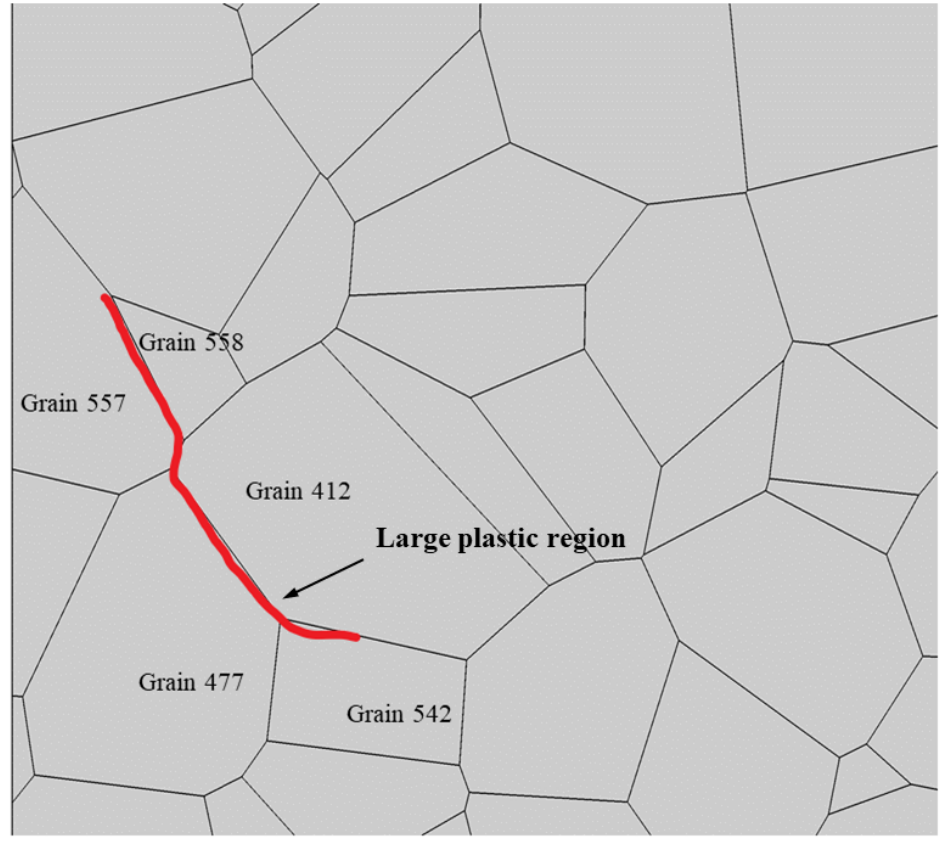

Figure 4. 29 Demonstration of adjacent grains in large plastic region at grain boundary.

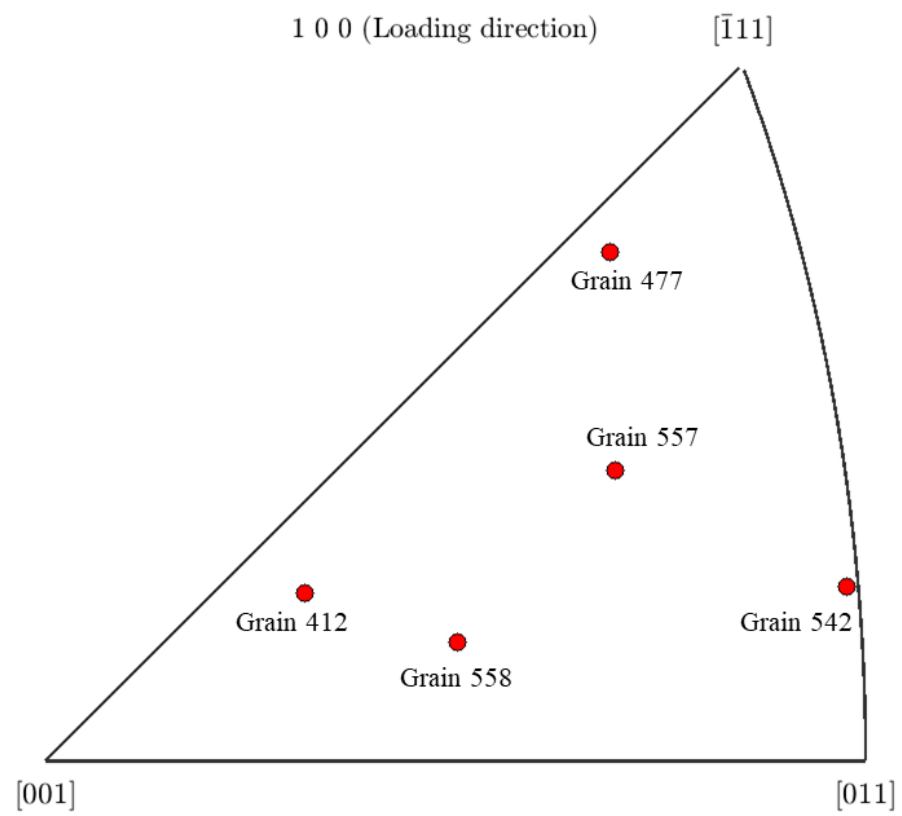

Figure 4. 30 Inverse pole figure of adjacent grains in large plastic region at grain boundary. 


\subsubsection{HCF life prediction}

Similarly, twenty (20) HCF simulations are performed for the HCF life prediction of Haynes 282 under the stress amplitudes of $400 \mathrm{MPa}$ and $415 \mathrm{MPa}$, which are notated as HCF- $\mathrm{T}_{\mathrm{i}}(\mathrm{i}=1,2,3 \ldots 20)$. Using the same procedures to the LCF case analysis, the localized plasticity and stress, the fatigue nucleation life and grain orientation effect are evaluated for the HCF case.

\subsubsection{Localized plasticity and stress under HCF}

The contour plots of stabilized plastic strain magnitude and Von Mises stress at the maximum strain level for $\mathrm{LCF}_{-} \mathrm{T}_{4}$ at the stress amplitude of $400 \mathrm{MPa}$ are shown in Figure 4.31 and Figure 4.32, respectively. It seems that even on the free surface of the RVE, most elements are not plastically deformed with zero value of local plastic strain magnitude. Plasticity is only observable in a few small regions. Such low level of plasticity is reasonable because the stress level of $400 \mathrm{MPa}$ is extremely close to the fatigue strength of Haynes $282(367.7 \mathrm{MPa})$ [4] below which the material is fully elastic at microscopic level. The local stress level is also shown to be significantly lower than that of the LCF case, with about $400 \mathrm{MPa}$ in most regions of the RVE. The maximum PEMAG value at the maximum strain range is found in an element on free surface $(z=0)$, as marked in Figure 4.33. Hence, the plastic strain range can be calculated: $\Delta \varepsilon_{p}=\varepsilon_{t}^{p}+\varepsilon_{c}^{p}=0.000547+0.000547=$ 0.001094. Other higher values displayed in the legend are discarded since the corresponding elements are on or too close to the planes affected by the imposed boundary 
condition.
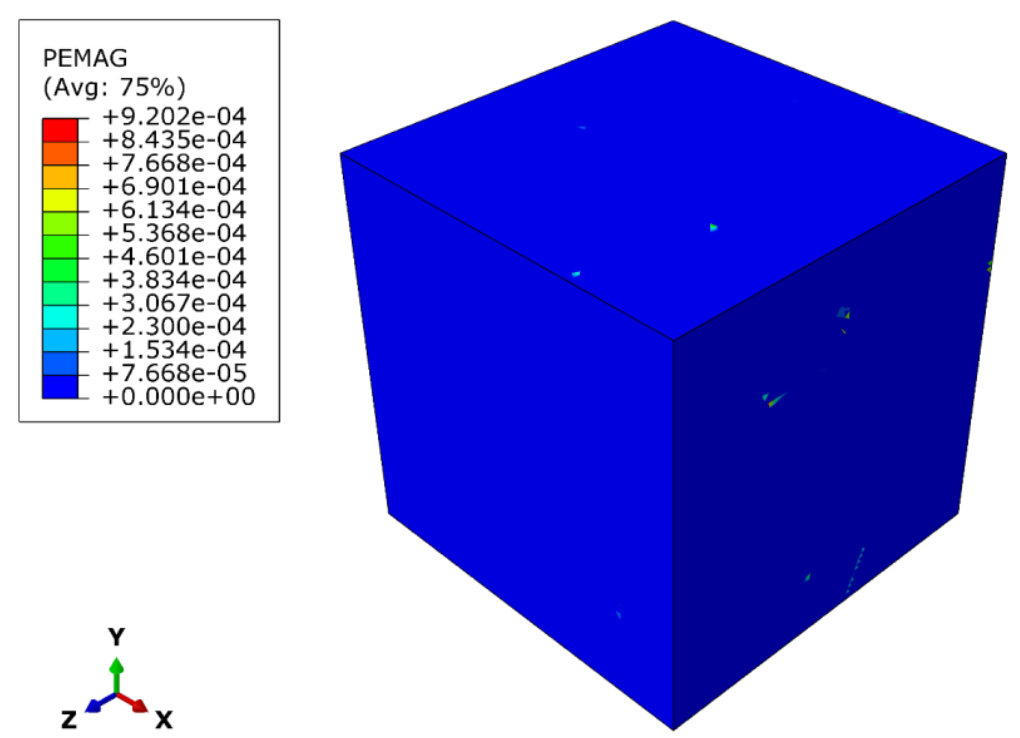

Figure 4. 31 Contour plots of plastic strain magnitude (PEMAG) at maximum strain level of 4 th cycle for $\mathrm{HCF}_{-} \mathrm{T}_{4}$ (stress amplitude $\left.\frac{\Delta \sigma}{2}=400 \mathrm{MPa}\right)$.
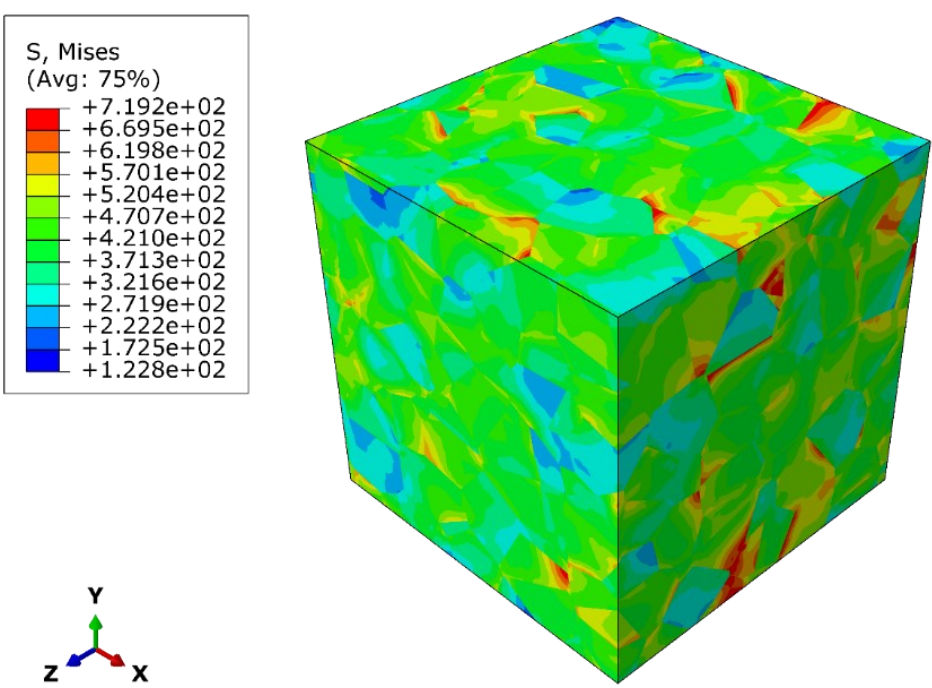

Figure 4. 32 Contour plots of Von Mises stress (S) at maximum strain level of 4th cycle for $\mathrm{HCF}_{-} \mathrm{T}_{4}$ (stress amplitude $\frac{\Delta \sigma}{2}=400 \mathrm{MPa}$ ). 


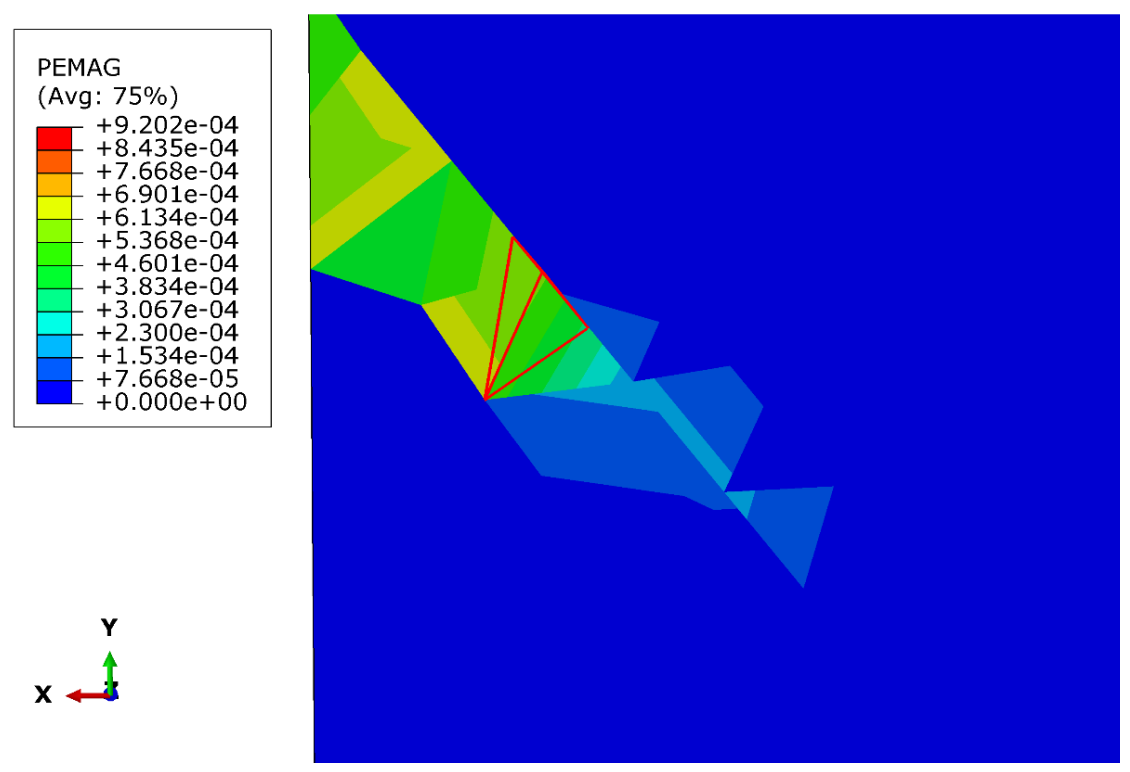

Figure 4. 33 Element with maximum plastic strain magnitude at maximum strain for HCF$\mathrm{T}_{4}$ (stress amplitude $\left.\frac{\Delta \sigma}{2}=400 \mathrm{MPa}\right)$.

\subsubsection{HCF crack nucleation life}

The calculated HCF lives of Haynes 282 for all 20 grain orientation distributions, along with corresponding maximum local plastic strain range $\Delta \varepsilon_{p}$, element number, and experimental HCF lives for stress amplitude $\frac{\Delta \sigma}{2}=415 \mathrm{MPa}$ and $400 \mathrm{MPa}$, are reported in Table 4.10 and Table 4.11, respectively. Figure 4.34 illustrates the comparison between the HCF life predictions and the experimental data of Haynes 282. The maximum local plastic strain range and calculated HCF life vary between 0.000494 to 0.001388 and 108271 to 854751 for the stress amplitude of $415 \mathrm{MPa} ; 0.000404$ to 0.001176 and 150826 to 1278000 for the stress amplitude of $400 \mathrm{MPa}$. Obviously, for both strain amplitudes, generally the maximum local plastic strain range and calculated HCF life are more scattered compared to those of the LCF case. Based on the data reported in Table 4.10 and 4.11, except for 
simulation 4 and simulation 10 with extremely long predicted HCF life, the predicted HCF life scattering, 3.62 and 4.52, for the stress amplitude of $415 \mathrm{MPa}$ and $400 \mathrm{MPa}$, respectively well coincides with that of the experimental data (3.42 and 3.28) [86]. In addition, the predicted HCF lives of Haynes 282 are on average lower than the experimental data by larger extent as compared to that for the LCF case. However, the average difference between the HCF life predictions and the experimental data is around $67.85 \%$ for stress amplitude of $415 \mathrm{MPa}$ and $62.93 \%$ for stress amplitude of $400 \mathrm{MPa}$, which are reasonably desirable results for HCF life prediction of materials.

Table 4. 10 Calculations of HCF life of Haynes 282 for stress amplitude $\frac{\Delta \sigma}{2}=415 \mathrm{MPa}$

\begin{tabular}{|c|c|c|c|c|}
\hline $\begin{array}{c}\text { Simulation } \\
\text { No }\end{array}$ & $\begin{array}{l}\text { Element } \\
\text { No }\end{array}$ & $\begin{array}{c}\text { Maximum local } \\
\text { plastic strain range }\end{array}$ & $\begin{array}{l}\text { Calculated } \\
\text { HCF life }\end{array}$ & $\begin{array}{c}\text { Experimental } \\
\text { HCF life }\end{array}$ \\
\hline 1 & 772162 & 0.001053 & 188236.09 & \multirow{4}{*}{372979} \\
\hline 2 & 63390 & 0.000609 & 562417.84 & \\
\hline 3 & 744461 & 0.001237 & 136417.22 & \\
\hline 4 & 1222575 & 0.001334 & 117177.67 & \\
\hline 5 & 92593 & 0.000729 & 391735.03 & \multirow{4}{*}{651762} \\
\hline 6 & 844747 & 0.001152 & 157126.61 & \\
\hline 7 & 914360 & 0.000989 & 213255.91 & \\
\hline 8 & 890559 & 0.001298 & 123806.74 & \\
\hline 9 & 220594 & 0.000864 & 279425.60 & \multirow{4}{*}{791768} \\
\hline 10 & 402721 & 0.000494 & 854751.32 & \\
\hline 11 & 870503 & 0.001166 & 153343.81 & \\
\hline 12 & 713490 & 0.001388 & 108271.64 & \\
\hline 13 & 695287 & 0.001116 & 167610.19 & \multirow{4}{*}{964966} \\
\hline 14 & 125537 & 0.000830 & 302518.30 & \\
\hline 15 & 986531 & 0.001128 & 163936.43 & \\
\hline 16 & 711451 & 0.001180 & 149806.15 & \\
\hline 17 & 512789 & 0.000777 & 345502.85 & \multirow{4}{*}{1176050} \\
\hline 18 & 671133 & 0.001019 & 200883.98 & \\
\hline 19 & 578269 & 0.000996 & 210268.88 & \\
\hline 20 & 498319 & 0.000892 & 262158.51 & \\
\hline
\end{tabular}


Table 4. 11 Calculations of HCF life of Haynes 282 for stress amplitude $\frac{\Delta \sigma}{2}=400 \mathrm{MPa}$

\begin{tabular}{|c|c|c|c|c|}
\hline $\begin{array}{l}\text { Simulation } \\
\text { No }\end{array}$ & $\begin{array}{l}\text { Element } \\
\text { No }\end{array}$ & $\begin{array}{c}\text { Maximum local } \\
\text { plastic strain range }\end{array}$ & $\begin{array}{l}\text { Calculated } \\
\text { HCF life }\end{array}$ & $\begin{array}{c}\text { Experimental } \\
\text { HCF life }\end{array}$ \\
\hline 1 & 772162 & 0.000892 & 263338.08 & \multirow{4}{*}{450366} \\
\hline 2 & 63390 & 0.000531 & 742573.25 & \\
\hline 3 & 744461 & 0.000998 & 209426.96 & \\
\hline 4 & 1222575 & 0.001094 & 174284.60 & \\
\hline 5 & 92593 & 0.000552 & 689554.02 & \multirow{4}{*}{886360} \\
\hline 6 & 844747 & 0.000994 & 211115.88 & \\
\hline 7 & 914360 & 0.000822 & 310217.27 & \\
\hline 8 & 890559 & 0.001102 & 171763.34 & \\
\hline 9 & 220594 & 0.000734 & 387169.87 & \multirow{4}{*}{1056006} \\
\hline 10 & 402721 & 0.000404 & 1278000.28 & \\
\hline 11 & 870503 & 0.001018 & 201278.84 & \\
\hline 12 & 713490 & 0.001176 & 150826.98 & \\
\hline 13 & 695287 & 0.000948 & 232100.99 & \multirow{4}{*}{1148910} \\
\hline 14 & 125537 & 0.000672 & 461907.62 & \\
\hline 15 & 986531 & 0.000934 & 239111.20 & \\
\hline 16 & 711451 & 0.001018 & 201278.84 & \\
\hline 17 & 512789 & 0.000616 & 549708.24 & \multirow{4}{*}{1475272} \\
\hline 18 & 671133 & 0.000866 & 278136.44 & \\
\hline 19 & 578269 & 0.000843 & 293520.56 & \\
\hline 20 & 498319 & 0.000728 & 393578.09 & \\
\hline
\end{tabular}




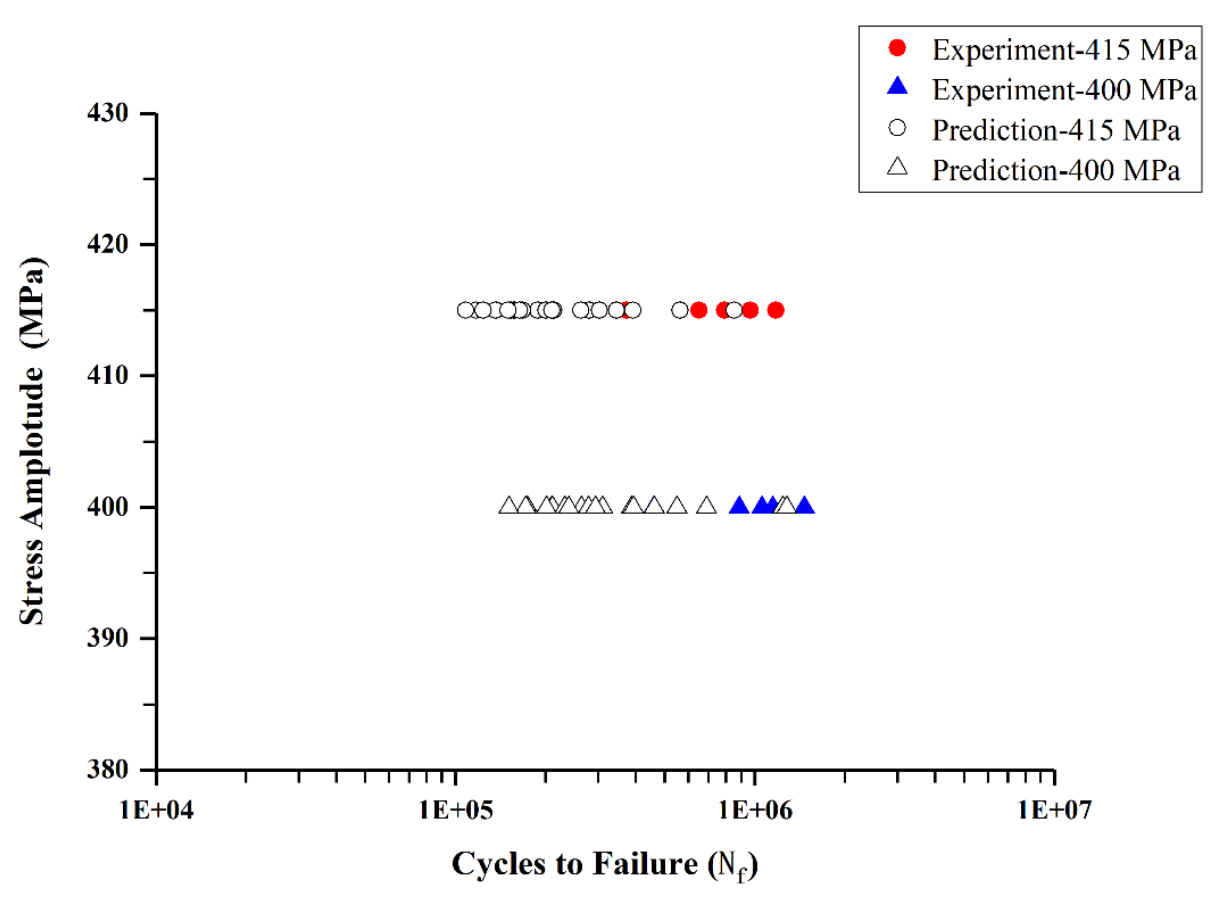

Figure 4. 34 Comparison between calculated HCF crack nucleation life and experimental total life for stress amplitudes of $415 \mathrm{MPa}$ and $400 \mathrm{MPa}$.

\subsubsection{Grain orientation effect on HCF}

The FEA simulation results shown in Figure 4.31 suggest that most elements are in fully elastic regime mainly due to the low global stress applied to the RVE model. Thus, it is pointless to analyze the correlation between the grains with zero plastic strain and their orientations. As a result, only the details of orientations of the grains with the maximum plastic strain range for all HCF simulations are presented in Table 4.12 and corresponding inverse pole figure and contour plots are shown in Figure 4.35 and Figure 4.36. In general, the results follow similar trend to that discovered in the LCF simulations where grains appear to be mainly oriented towards the family of [ [ $\left.\begin{array}{lll}1 & 1 & 1\end{array}\right]$ direction, but the difference is that more grains locate closer to the family of [ $\left[\begin{array}{lll}0 & 1 & 1\end{array}\right]$ direction compared to the LCF case. 
The possible reason for this behavior is that the yielding point of nickel single crystal is higher in $\left[\begin{array}{lll}1 & 1 & 1\end{array}\right]$ direction than in $\left[\begin{array}{lll}0 & 1 & 1\end{array}\right]$ direction. At very low global stress level the local plasticity of nickel single crystal is very sensitive to yield strength, which represents the onset of plastic deformation. 
Table 4. 12 Orientations of grains with maximum plastic strain range for HCF simulations

\begin{tabular}{|c|c|c|c|c|c|c|c|}
\hline Test No & Grain No & $\mathrm{h}$ & $\mathrm{k}$ & 1 & $\varphi\left({ }^{\circ}\right)$ & $\theta\left(^{\circ}\right)$ & $\psi\left({ }^{\circ}\right)$ \\
\hline 1 & 380 & -4 & 5 & 8 & 155.749 & 76.5882 & 127.53 \\
\hline 2 & 99 & -2 & 8 & 9 & 12.3965 & 127.042 & 139.225 \\
\hline 3 & 360 & -6 & 11 & 12 & 223.661 & 82.6965 & 144.128 \\
\hline 4 & 557 & -1 & 12 & 12 & 54.858 & 61.5384 & 320.897 \\
\hline 5 & 107 & -5 & 10 & 11 & 52.4578 & 155.86 & 92.0123 \\
\hline 6 & 401 & -10 & 10 & 11 & 82.6316 & 35.1704 & 230.438 \\
\hline 7 & 274 & -5 & 7 & 9 & 328.834 & 50.3493 & 253.638 \\
\hline 8 & 413 & -2 & 2 & 3 & 311.989 & 99.7592 & 125.412 \\
\hline 9 & 168 & 0 & 1 & 1 & 51.4855 & 113.213 & 27.5563 \\
\hline 10 & 243 & -5 & 6 & 7 & 315.347 & 73.6257 & 144.985 \\
\hline 11 & 407 & -11 & 11 & 12 & 305.464 & 44.2183 & 267.877 \\
\hline 12 & 350 & -4 & 5 & 5 & 37.0043 & 125.046 & 158.67 \\
\hline 13 & 343 & -7 & 9 & 10 & 277.913 & 36.8019 & 114.519 \\
\hline 14 & 123 & -1 & 5 & 6 & 56.8306 & 65.6826 & 69.4932 \\
\hline 15 & 462 & -7 & 9 & 10 & 38.5731 & 69.5719 & 39.4995 \\
\hline 16 & 349 & -4 & 5 & 8 & 50.7841 & 88.0157 & 227.784 \\
\hline 17 & 271 & -4 & 7 & 8 & 311.424 & 70.4106 & 259.312 \\
\hline 18 & 363 & -2 & 5 & 6 & 289.36 & 127.623 & 187.262 \\
\hline 19 & 424 & -11 & 11 & 12 & 73.4222 & 34.8265 & 63.58 \\
\hline 20 & 269 & -6 & 7 & 8 & 305.209 & 126.987 & 359.437 \\
\hline
\end{tabular}




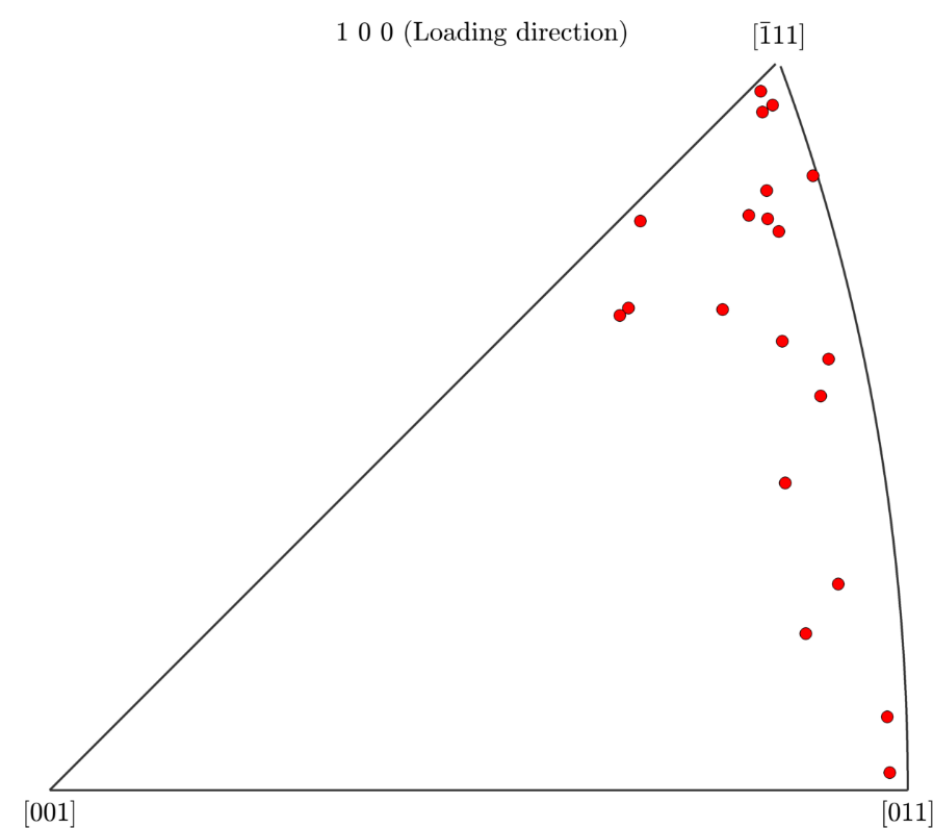

Figure 4. 35 Inverse pole figure of grains with maximum plastic strain range for all HCF simulations.

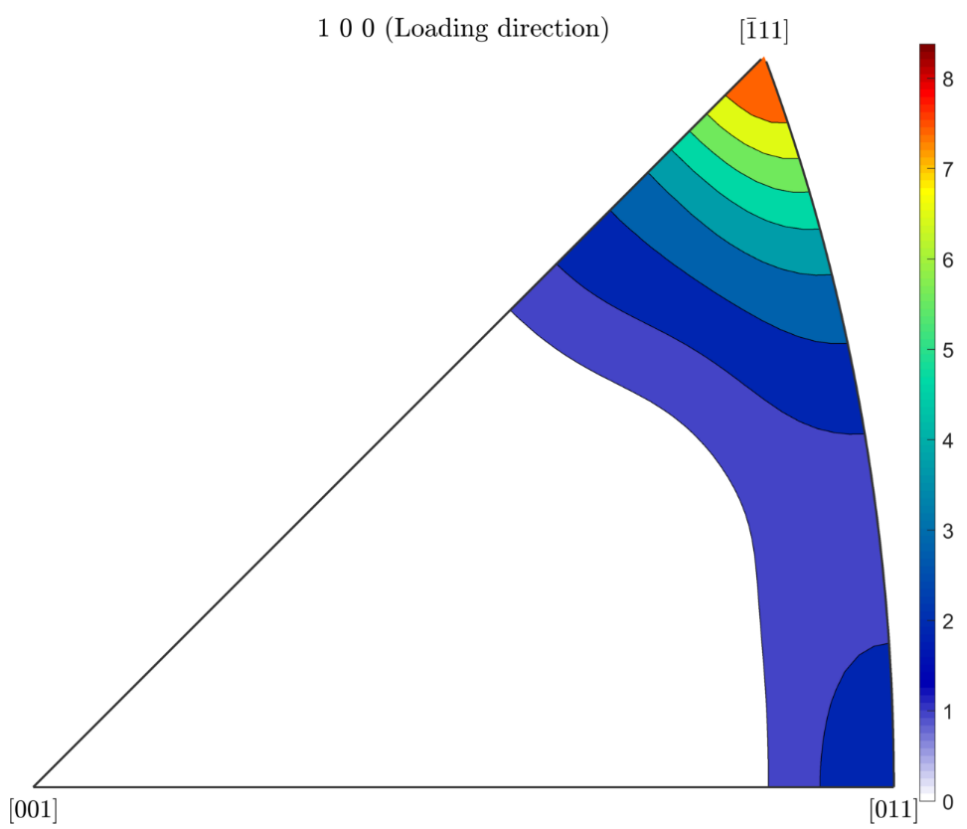

Figure 4. 36 Contour plots of orientations of grains with maximum plastic strain range for all HCF simulations. 


\section{Chapter 5 Conclusions and Future Work}

\subsection{Summary of the Research}

In this research, an analytical fatigue life prediction model, named the TMW model, is applied on eight different structural materials including aluminum alloy, alloy steel, titanium alloy and nickel-based alloy. The predicted fatigue lives from the TMW model for the low cycle fatigue (LCF) region using the strain-based formula, Eq (3.1), and for the high cycle fatigue (HCF) region according to the stress-based formula, Eq (3.2), are compared with the experimental data and results obtained from the Coffin-Manson relation and from the Basquin relation, respectively, which were all derived from experimental data fitting. The required material properties and the TMW parameters for the fatigue life predictions of the eight selected alloys and steels are determined either based on existing experimental data in literature or from the calculations utilizing relevant data obtained from various sources. The validity of the TMW model is analyzed based on the comparison results.

Furthermore, to facilitate the application of the TMW model, an attempt is made to mathematically describe the correlation between the surface roughness factor $R_{S}$ in the TMW model and the profile roughness parameter $R_{a}$ for LCF, Eq (3.1) and HCF, Eq (3.2), separately, because the microstructure-dependent parameter, the lattice resistance $\sigma_{0}$, in Eq (3.2) for HCF is also surface roughness-dependent, using the fatigue testing data of 
aluminum alloy and medium-carbon steel available in literature so that the surface roughness factor $R_{s}$ in the TMW model can be substituted by the profile roughness parameter $R_{a}$ which can be experimentally determined for a given surface. The influence of the surface roughness of aluminum alloy and medium-carbon steel components on their fatigue crack nucleation life is investigated for both LCF and HCF. The revised TMW model, i.e., strain-based fatigue life formula and the stress-based fatigue life formula for LCF and HCF, respectively, is obtained with the surface roughness factor $R_{S}$ replaced by the profile roughness parameter $R_{a}$.

Finally, with the intention to economically assess the fatigue life scattering mainly resulted from microstructural inhomogeneity (grain orientation is considered in this study), FEA microstructure modeling is conducted for fatigue design of structural materials, taking nickel-based alloy Haynes 282 as an example. The FEA simulation on this alloy is performed at multiple strain ranges from LCF to HCF at different strain and stress amplitudes. To simulate the realistic microstructure of Haynes 282, a material representative volume element (RVE) is created using Voronoi tessellation method with Python Scripts. Twenty random grain orientation distribution functions are assigned to the material RVE so as to evaluate the effect of microstructural inhomogeneity on the fatigue crack nucleation life of Haynes 282 in both LCF simulations (strain ranges at $0.84 \%$ and $0.93 \%$ ) and HCF simulations (stress amplitudes at $400 \mathrm{MPa}$ and $415 \mathrm{MPa}$ ). The material properties the FEA model requires including anisotropic elasticity and plasticity constants 
are determined for nickel-based alloy. The hysteresis behavior of the material is considered to determine the reference yielding strength and kinematic hardening of Haynes 282. The FEA simulation is combined with the TMW model to predict the fatigue crack nucleation life of Haynes 282 under LCF or HCF. The predicted results are analyzed and compared with the experimental data of Haynes 282 from literature.

\subsection{Conclusions from Present Work}

From the analysis and application of the TMW model on selected aluminum alloys, alloy steels, titanium alloy and nickel-based alloys, and the fatigue life prediction of Haynes 282 using a combined approach of the FEA simulation for microstructural inhomogeneity (grain orientation) and the TMW model, the following conclusions can be drawn from the this research.

1. The fatigue life predictions from the TMW model on selected alloys and steels reveal that the TMW model, in general, displays effectiveness for fatigue life prediction of common engineering structural materials when compared with the experimental data and results obtained from the Coffin-Manson-Basquin relations. In particular, the strain-based formula $\mathrm{Eq}(3.1)$ is favorable for the LCF region (plastic strain above 0.001) while the stress-based formula Eq (3.2) is suitable for the HCF region where the deformation is macroscopically elastic below the yield point.

2. The mathematical relations are derived to express the surface roughness factor $R_{S}$ in the 
TMW model in terms of the arithmetical mean deviation of the assessed profile $R_{a}$. Since the latter is obtainable experimentally, this substitution makes the TMW model more applicable.

3. Surface roughness of components/structures affects their fatigue life differently for LCF and HCF, but in the TMW model for both the strain-based formula, Eq (3.1), and the stressbased formula Eq (3.2), $R_{S}$ and $R_{a}$ have been proven to be exponentially correlated and the exponent varies with materials. In addition, a linear relation appears to be effective in describing lattice resistance $\sigma_{0}$ with surface roughness profile $R_{a}$ for Eq (3.2).

4. In the FEA simulation, based on the assumption that fatigue crack first nucleates in the regions with the largest plasticity, potential fatigue crack nucleation sites are prone to occur at grain boundaries right on or close to the free surface of the RVE, which coincides with experimental observations.

5. The predicted fatigue nucleation life of Haynes 282 by the TMW model using the maximum plastic strain range determined from the FEA simulation generally agrees well with the experimental data in literature, but discrepancy does exist between the modeling and experimental results, which is lower than $50 \%$ on average for LCF, and about $66.5 \%$ for HCF. One of the reasons for this difference can be attributed to the fact that the testing data refer to the total fatigue life, i.e., the sum of fatigue crack nucleation fraction and fatigue crack propagation fraction, while the TMW model only predicts the fatigue crack 
nucleation life.

6. The TMW model, combined with the microstructure FEA model with varied grain orientation distribution, successfully provides reasonably good predictions of the LCF and HCF life as well as prediction scattering for Haynes 282, which are found to be close to that reported data in literature.

7. The study of grain orientation effect on the fatigue life of Haynes 282 indicates that for both HCF and LCF, the simulated fatigue crack nucleation is more likely to occur in the grains of $\left[\begin{array}{lll}1 & 1 & 1\end{array}\right]$ family. In contrast, the grains with the least probability of fatigue crack nucleation tend to be oriented towards $\left[\begin{array}{lll}0 & 0 & 1\end{array}\right]$ family. This result can be justified by the

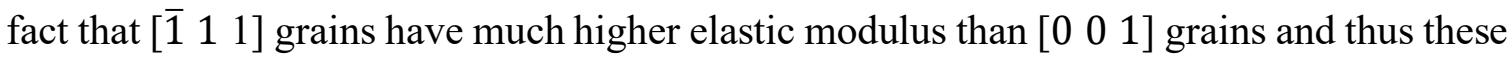
grains endure higher stress than $\left[\begin{array}{lll}0 & 0 & 1\end{array}\right]$ grains, which contributes to plastic deformation. Moreover, the yield strength is lower in [ [ $\left.\begin{array}{lll}1 & 1 & 1\end{array}\right]$ grains than in $\left[\begin{array}{lll}0 & 0 & 1\end{array}\right]$ grains.

8. Fatigue crack nucleation is found to preferentially occur at the grain boundaries with high grain misalignment, which acts as an obstacle of dislocation motion and thus results in stress concentration.

\subsection{Significant Contributions of the Research}

In addition to the conclusions stated above, the significant contributions of this research can be highlighted as follows: 
1. Although the TMW model has been developed, it has only been applied on a few pure metals such as iron and copper and a limited number of alloys for LCF life prediction. The present research extends the TMW model application to a wide range of materials including aluminum alloys, alloy steels, titanium alloy and nickel-based alloy. The most pronounced is that the TMW model is validated for both the strain-based LCF region and the stressbased HCF region by using it to predict the fatigue crack nucleation lives of various materials and comparing the predicted lives with the results calculated using the CoffinManson-Basquin relations which were derived from experimental data fitting.

2. Although the TMW model includes the surface roughness factor, it is unknown and cannot be quantified. As a result, the fatigue life predicted by the TMW model may deviate from the real scenario due to assumed value for the surface roughness factor. In the present study, the mathematical expressions for the surface roughness factor $R_{S}$ in the TMW model, in terms of the arithmetical mean deviation of the assessed profile $R_{a}$ which is experimentally obtainable, are developed for LCF and HCF, separately. This substitution improves the TMW model with increasing applicability and prediction accuracy.

3. Microstructure-based computational fatigue design approach for materials/structures has been developed for years, but the simulation of microstructural inhomogeneity, e.g., grain orientation, is often made in 2D model with a limited number of elements, which definitely cannot reflect the real characteristics of the microstructure. The FEA simulation study in this research uses a 3D microstructure model, i.e., a material representative volume element 
(RVE), to evaluate the effect of grain orientation on the fatigue crack nucleation life of Haynes 282, with 20 random grain orientation distribution functions assigned to the RVE in both HCF and LCF simulations. Since no such research on Haynes 282 has been reported in literature, the outcomes of this research provide valuable technical support for this alloy in potential industrial applications.

\subsection{Suggested Future Work}

The present work has demonstrated that the combination of the TMW model and finite element microstructural modeling is a very promising approach to analytically predicting the fatigue crack nucleation life of structural materials at room temperature. However, in real application situations of many structural materials such as nickel-based alloys, for instance, the service temperature of gas turbine engines can go up to $1000{ }^{\circ} \mathrm{C}$. To simulate the true working condition of Haynes 282 alloy which is a popular material used for gas turbine engine components, there is a need to extend the current fatigue design from room temperature to elevated temperatures. In this case, creep performance of Haynes 282 may also be considered.

In the present work the effect of grain orientation on the fatigue nucleation life of Haynes 282 has been evaluated and discussed. However, there are other microstructural inhomogeneities such as grain size and grain shape that can also affect the fatigue crack nucleation of the material. Therefore, more numerical studies are needed to study the 
effects of other microstructural inhomogeneities, e.g., grain morphology in this scope.

Since it is very difficult to accurately determine the fatigue crack nucleation life of materials experimentally, in the present study, the predicted fatigue crack nucleation lives are simply compared with the total fatigue life determined experimentally to check the validity of the TMW model. In reality, there is also a period of crack growth before fatigue fracture occurs. Therefore, in the future work, microstructure-based crack growth simulation should be performed, linking to the present crack nucleation simulation, thus to achieve the total fatigue life prediction, which is very important for structural integrity assessment rather than coupon analysis only.

Although the TMW model has shown the capability of providing an effective computation method to estimate fatigue life of structural materials, there are still some factors such as loading spectra and residual stress, etc. not accounted in the model. Therefore, in the future work the model will be further improved by taking into account loading condition effect via either physical experiment or theoretical analysis. 


\section{References}

[1] F. C. Campbell, “Chapter 14: Fatigue," Elem. Metall. Eng. Alloy., pp. 243-265, 2008.

[2] Z. Zhang, G. Yang, and K. Hu, "Prediction of fatigue crack growth in gas turbine engine blades using acoustic emission," Sensors (Switzerland), vol. 18, no. 5, 2018, doi: $10.3390 / \mathrm{s} 18051321$.

[3] W. Będkowski, "Assessment of the fatigue life of machine components under service loading - A review of selected problems," J. Theor. Appl. Mech., vol. 52, no. 2, pp. 443-458, 2014.

[4] Flight Safety Foundation, "Fatigue Crack Cited in BA 777 Uncontained Engine Failure, Fire,” 2018. https://flightsafety.org/fatigue-crack-led-to-777-engine-failure-fire/ (accessed Jun. 21, 2018).

[5] B. Zhang, "One person is dead after a major engine failure led a Southwest plane to make a terrifying emergency landing in Philadelphia," 2018. https://www.businessinsider.com/southwest-airlines-engine-failure-terrifying-emergencylanding-2018-4 (accessed Apr. 17, 2018).

[6] A. Drews, "Standard Test Method for Strain-Controlled Fatigue Testing," Man. Hydrocarb. Anal. 6th Ed., pp. 545-545-3, 2008, doi: 10.1520/mnl10913m.

[7] J. Schijve, Fatigue of Structures and Materials, 2nd ed. Springer Science \& Business Media, 2008.

[8] X. Wu, Deformation and Evolution of Life in Crystalline Materials, 1st ed. CRC Press, 2019.

[9] X. Wu, "On Tanaka-Mura's fatigue crack nucleation model and validation," Fatigue Fract. Eng. Mater. Struct., vol. 41, no. 4, pp. 894-899, 2018, doi: $10.1111 /$ ffe. 12736 .

[10] S. Mahajan, Encyclopedia of Materials: Science and Technology, 1st ed. Elsevier, 2001 .

[11] S. R. Yeratapally et al., "Microstructure based fatigue life prediction framework 
for polycrystalline nickel-base superalloys with emphasis on the role played by twin boundaries in crack initiation," Acta Mater., vol. 107, pp. 152-167, 2016, doi: 10.1016/j.actamat.2016.01.038.

[12] G. Olivares, J. F. Acosta, and V. Yadav, “Certification by Analysis I and II,” 2010.

[13] R. I. Stephens et al., Metal Fatigue in Engineering, 2nd ed. New York: John Wiley\&Sons,Inc, 2001.

[14] J. A. Bannantine, J. J. Comer, and J. L. Handrock, Fundamentals of Metal Fatigue Analysis. Englewood Cliffs,N.J.: Prentice Hall, 1990.

[15] ASM International.Handbook Committee, Fatigue and Fracture. Materials Park, OH:ASM International, 1996.

[16] Q. Xin, "Durability and reliability in diesel engine system design," in Diesel Engine System Design, 2011, pp. 113-202.

[17] M. D. Sangid, “The physics of fatigue crack initiation," Int. J. Fatigue, vol. 57, pp. 58-72, 2013, doi: 10.1016/j.ijfatigue.2012.10.009.

[18] S. Kumar and W. A. Curtin, "Crack interaction with microstructure Designing microstructure for damage tolerance requires a detailed," Mater. Today, vol. 10, no. 9, pp. 34-44, 2007, doi: 10.1016/S1369-7021(07)70207-9.

[19] J. Schijve, Fatigue of Structures and Materials, 2nd ed. Springer Science \& Business Media, 2008.

[20] L. Molent and B. Dixon, "Airframe metal fatigue revisited," Int. J. Fatigue, vol. 131, no. October 2019, p. 105323, 2020, doi: 10.1016/j.ijfatigue.2019.105323.

[21] H. J. Grover, Fatigue of Aircraft Structures. 1966.

[22] L. Kunz and P. Luka, "Small cracks - nucleation, growth and implication to fatigue life," vol. 25, pp. 855-862, 2003, doi: 10.1016/S0142-1123(03)00133-6.

[23] C. Li et al., "A Transition Size of Dividing Crack Initiation and Propagation Phases and the Fatigue Total Life Prediction Approach," J. Fail. Anal. Prev., vol. 19, no. 5, pp. 1380-1388, 2019, doi: 10.1007/s11668-019-00726-7.

[24] M. H. El Haddad, K. N. Smith, and T. H. Topper, "Fatigue crack propagation of short cracks," J. Eng. Mater. Technol. Trans. ASME, vol. 101, no. 1, pp. 42-46, 1979, doi: 
10.1115/1.3443647.

[25] R. Buckson, "Evaluation of Fatigue Behaviour of Laser Beam Welded Aerospace Superalloy Haynes 282,” 2015.

[26] J. R. Hook and H. E. Hall, Solid State Physics, 2nd ed. Wiley, 1991.

[27] G. Marquis and J. Solin, Fatigue Design of Components, 1st ed. Elsevier Science, 1997.

[28] M. H. Shen and S. R. Akanda, "An Energy-Based Approach to Determine the Fatigue Strength and Ductility Parameters for Life Assessment of Turbine Materials," $J$. Eng. Gas Turbines Power, vol. 137, no. 7, pp. 1-7, 2015, doi: 10.1115/1.4029204.

[29] Q. Y. Wang et al., "Effect of inclusion on subsurface crack initiation and gigacycle fatigue strength," vol. 24, pp. 1269-1274, 2002.

[30] X. J. Wu, A. K. Koul, and A. S. Krausz, "A transgranular fatigue crack growth model based on restricted slip reversibility," Metall. Trans. A, vol. 24, no. 6, pp. 13731380, 1993, doi: 10.1007/BF02668205.

[31] B. Ma and C. Laird, "Overview of Fatigue Behavior in Copper Single CrystalsIII. Interpretation of Crack Growth Kinetics and a New Approach to Predict Fatigue Life Based on Crack Population Density in Specimens Cycled at Constant Strain Amplitude," vol. 37, no. 2, pp. 349-355, 1989.

[32] M. Makkonen, "Predicting the total fatigue life in metals," Int. J. Fatigue, vol. 31, no. 7, pp. 1163-1175, 2009, doi: 10.1016/j.ijfatigue.2008.12.008.

[33] N. F. Mott, "A Theory of the Origin of Fatigue Cracks," Acta Metall., vol. 6, no. 3, pp. 195-197, 1958, doi: https://doi.org/10.1016/0001-6160(58)90007-5.

[34] E. Santecchia et al., "A Review on Fatigue Life Prediction Methods for Metals," vol. 2016, p. 26, 2016, doi: https://doi.org/10.1155/2016/9573524.

[35] K. Tanaka and T. Mura, "A Dislocation Model for Fatigue Crack Initiation," vol. 48, no. 81, pp. 97-103, 1981.

[36] N. I. Muskhelishvili, Singular Integral Equations. Dover Publications, 2013.

[37] R. G. Tryon and T. A. Cruse, "A reliability-based model to predict scatter in fatigue crack nucleation life," Fatigue Fract. Eng. Mater. Struct., vol. 21, no. 3, pp. 257-267, 1998, 
doi: 10.1046/j.1460-2695.1998.00024.x.

[38] J. Kramberger, N. Jezernik, P. Göncz, and S. Glodež, "Extension of the TanakaMura model for fatigue crack initiation in thermally cut martensitic steels," Eng. Fract. Mech., vol. 77, no. 11, pp. 2040-2050, 2010, doi: 10.1016/j.engfracmech.2009.12.003.

[39] A. Bruckner-Foit and X. Huang, "On the determination of material parameters in crack initiation laws," Fatigue Fract. Eng. Mater. Struct., pp. 980-988, 2008, doi: 10.1111/j.1460-2695.2008.01287.x.

[40] N. Jezernik et al., "Numerical modelling of fatigue crack initiation and growth of martensitic steels," Fatigue Fract. Eng. Mater. Struct., vol. 33, no. 11, pp. 714-723, 2010, doi: 10.1111/j.1460-2695.2010.01482.x.

[41] W. R. Tyson, "Surface Free Energies of Sold Metals Estimation From Liquid Surface Tension Measurements," Surf. Sci., vol. 62, pp. 267-276, 1977.

[42] G.Venkataraman, Y.W.Chung, and A. T.Mura, "Application of Minimum Energy Formalism in a Multiple Slip Band Model for Fatigue--II . Crack Nucleation and Derivation of a Generalised Coffin-Manson Law," Acta Metall. Mater., vol. 39, no. II, pp. 2631-2638, 1991, doi: https://doi.org/10.1016/0956-7151(91)90079-G.

[43] J. Wareing and H. G. Vaughan, "Influence of surface finish on low-cycle fatigue characteristics of Type 316 stainless steel at $400^{\circ}$ C," Met. Sci., pp. 1-8, 1979, doi: 10.1179/030634579790434150.

[44] H. Mughrabi and H. W. Höppel, "Cyclic deformation and fatigue properties of very fine-grained metals and alloys," Int. J. Fatigue, vol. 32, no. 9, pp. 1413-1427, 2010, doi: 10.1016/j.ijfatigue.2009.10.007.

[45] Z. F. Zhang, H. C. Gu, and X. L. Tan, "Low-cycle fatigue behaviors of commercialpurity titanium," Mater. Sci. Eng. A, vol. 252, no. 1, pp. 85-92, 1998, doi: 10.1016/S09215093(98)00650-9.

[46] M. C. Tumgsteh and E. Procedures, "d CONF-810831—82 " 8," pp. 0-4.

[47] B. A. Lerch, N. Jayaraman, and S. D. Antolovich, "A study of fatigue damage mechanisms in Waspaloy from 25 to $800^{\circ} \mathrm{C}$," Mater. Sci. Eng., vol. 66, no. 2, pp. 151-166, 1984, doi: 10.1016/0025-5416(84)90177-0.

[48] J. Reuchet and L. Rémy, "High Temperature Fatigue Behaviour of a Cast CobaltBase Superalloy," Fatigue Fract. Eng. Mater. Struct., vol. 2, no. 1, pp. 51-62, 1979, doi: 
10.1111/j.1460-2695.1979.tb01342.x.

[49] J. Reuchet and L. Remy, "High temperature low cycle fatigue of MAR-M 509 superalloy II: The influence of oxidation at high temperatures," Mater. Sci. Eng., vol. 58, no. 1, pp. 33-42, 1983, doi: 10.1016/0025-5416(83)90135-0.

[50] "Practice for conducting force controlled constant amplitude axial fatigue tests of metallic materials," ASTM B. Stand., vol. i, pp. 1-6, 2015, doi: 10.1520/E0466-15.2.

[51] P. Safarian, "Requirements of Certification by Analysis," 2018, [Online]. Available: https://www.nafems.org/2018/americas/keynotes/safarian/.

[52] F. Briffod, T. Shiraiwa, and M. Enoki, "Fatigue crack initiation simulation in pure iron polycrystalline aggregate," Mater. Trans., vol. 57, no. 10, pp. 1741-1746, 2016, doi: 10.2320/matertrans.M2016216.

[53] K. S. Chan, "A microstructure-based fatigue-crack-initiation model," Metall. Mater. Trans. A Phys. Metall. Mater. Sci., vol. 34, no. 1, pp. 43-58, 2003, doi: 10.1007/s11661003-0207-9.

[54] N. A. Giang et al., "A model for predicting crack initiation in forged M3:2 tool steel under high cycle fatigue," Int. J. Fract., vol. 187, no. 1, pp. 145-158, 2014, doi: 10.1007/s10704-013-9927-z.

[55] P. Brøndsted and P. Skov-Hansen, "Fatigue properties of high-strength materials used in cold-forging tools," Int. J. Fatigue, vol. 20, no. 5, pp. 373-381, 1998, doi: 10.1016/S0142-1123(98)00006-1.

[56] T. Seiichiro, S. Moe, and F. Riccardo, "Prediction of fatigue crack initiation life of aluminium alloy joints using cyclic elasto-plasticity FEM analysis," MATEC Web Conf., vol. 165, pp. 1-6, 2018, doi: 10.1051/matecconf/201816514012.

[57] H. Hirukawa et al., "Fatigue Properties of Butt-Welded joints for 5083-O Aluminum Alloy," Trans. Japan Soc. Mech. Eng. Ser. A, vol. 58, pp. 676-682, 1992, doi: https://doi.org/10.1299/kikaia.58.676.

[58] R. E. Newnham, Properties of Materials: Anisotropy, Symmetry, Structure, 1st ed. Oxford University Press, 2005.

[59] T. C. T. Ting, Anisotropic Elasticity: Theory And Applications. New York: Oxford University Press, 1996. 
[60] P. P. Benham, R. J. Crawford, and C. G. Armstrong, Mechanics of Engineering Materials, 2nd ed. Longman Group Ltd.,Harlow, 1996.

[61] J. Zhao et al., "Finite element analysis of a notch root semi-elliptical crack in single crystal superalloy," Eng. Fract. Mech., vol. 71, no. 13-14, pp. 1873-1890, 2004, doi: 10.1016/j.engfracmech.2003.11.008.

[62] R.Hill, "A theory of the yielding and plastic flow of anisotropic metals," Proc. R. Soc. London. Ser. A. Math. Phys. Sci., vol. 193, no. 1033, pp. 281-297, 1948, doi: 10.1098/rspa.1948.0045.

[63] W. F. Hosford, Solid Mechanics., Reprint ed. Cambridge University Press, 2014.

[64] Allan F.Bower, Applied Mechanics of Solids, CRC Press. 2009.

[65] "Introduction to EBSD." https://cfamm.ucr.edu/document/ebsd-intro.

[66] M. Knezevic et al., "A numerical procedure enabling accurate descriptions of strain rate-sensitive flow of polycrystals within crystal visco-plasticity theory," Comput. Methods Appl. Mech. Eng., vol. 308, pp. 468-482, 2016, doi: 10.1016/j.cma.2016.05.025.

[67] Z. Y. Ren and Q. S. Zheng, "A Quantitative study of minimum sizes of representative volume elements of cubic polycrystals - Numerical experiments," J. Mech. Phys. Solids, vol. 50, no. 4, pp. 881-893, 2002, doi: 10.1016/S0022-5096(01)00102-8.

[68] X. Huang, "Simulation on the process of fatigue crack initiation in a martensitic stainless steel," Stress Int. J. Biol. Stress, 2007.

[69] J. Lemaitre, "Formulation and Identification of Damage Kinetic Constitutive Equations," in Continuum Damage Mechanics Theory and Application, Springer, Vienna, 1987, pp. 37-89.

[70] S. Weyer et al., "Automatic finite element meshing of planar Voronoi tessellations," Eng. Fract. Mech., vol. 69, no. 8, pp. 945-958, 2002, doi: 10.1016/S0013-7944(01)001242.

[71] Q. Du, V. Faber, and M. Gunzburger, "Centroidal Voronoi tessellations: Applications and algorithms," SIAM Rev., vol. 41, no. 4, pp. 637-676, 1999, doi: $10.1137 / \mathrm{S} 0036144599352836$.

[72] J. Burns, "Centroidal voronoi tessellation," 2009. https://www.whitman.edu/Documents/Academics/Mathematics/burns.pdf. 
[73] C. T. Sims, "A History of Superalloy Metallurgy for Superalloy Metallurgists," pp. 399-419, 2012, doi: 10.7449/1984/superalloys_1984_399_419.

[74] W. O. Alexander, Ed., Metallurgical Achievements:Selection of Papers Presented at the Birmingham Metallurgical Societys Diamondjubilee Session 1963-1964, 1st ed. Pergamon Press, 1965.

[75] H. S. Mali and D. R. Unune, "Machinability of Nickel-Based Superalloys: An Overview," Mater. Sci. Mater. Eng., 2017, doi: 10.1016/B978-0-12-803581-8.09817-9.

[76] ASM International.Handbook Committee, Properties and Selection: Irons,Steels, and High-Performance Alloys. Materials Park,OH:ASM International, 2010.

[77] L. M. Pike, "Development of a Fabricable Gamma-Prime ( $\gamma$ ') Strengthened Superalloy,” Miner. Met. Mater. Soc., pp. 191-200, 2008.

[78] M. J. Donachie, "Relationship of Properties to Microstructure in Superalloys," in American Society for Metals, Superalloys, 1984, pp. 102-111.

[79] N. E. Ugodilinwa, "Analysis and Constitutive Modeling of Dynamic Impact Behaviour of Haynes 282 Superalloy,” University of Manitoba, 2018.

[80] Haynes® International Inc., "Haynes ${ }^{\circledR 282}{ }^{\circledR}$ Alloy, Datasheet," 2006, [Online]. Available: http://www.haynesintl.com/pdf/h3173.pdf.

[81] G. Włoch, D. Zasada, and R. M. Purgert, "Microstructure and Hardness Evolution in Haynes 282 Nickel-Based Superalloy During Multi-variant Aging Heat Treatment," vol. 28, no. July, pp. 3844-3851, 2019, doi: 10.1007/s11665-019-3886-0.

[82] Haynes ${ }^{\circledR}$ International Inc., “From 10-K.Annal Report," 2020. doi: 10.1017/CBO9781107415324.004.

[83] K. Barat et al., "High-Temperature Low-Cycle Fatigue Behavior in HAYNES 282: Influence of Initial Microstructure," Metall. Mater. Trans. A Phys. Metall. Mater. Sci., vol. 49, no. 10, pp. 5211-5226, 2018, doi: 10.1007/s11661-018-4760-7.

[84] J. He, R. Sandström, and S. Notargiacomo, "Low-Cycle Fatigue Properties of a Nickel-Based Superalloy Haynes 282 for Heavy Components," J. Mater. Eng. Perform., vol. 26, no. 5, pp. 2257-2263, 2017, doi: 10.1007/s11665-017-2586-x.

[85] R. A. Buckson and O. A. Ojo, "Cyclic deformation characteristics and fatigue crack growth behaviour of a newly developed aerospace superalloy Haynes 282," Mater. Sci. 
Eng. A, vol. 555, pp. 63-70, 2012, doi: 10.1016/j.msea.2012.06.034.

[86] M. Yang et al., "High cycle fatigue behavior of HAYNES282 superalloy," Miner. Met. Mater. Ser., no. 9783319523330, pp. 203-212, 2017, doi: 10.1007/978-3-319-523330_19.

[87] A. H. Noroozi, G. Glinka, and S. Lambert, "A two parameter driving force for fatigue crack growth analysis," Int. J. Fatigue, vol. 27, no. 10-12, pp. 1277-1296, 2005, doi: 10.1016/j.ijfatigue.2005.07.002.

[88] M. A. Meggiolaro and J. T. P. Castro, "Statistical evaluation of strain-life fatigue crack initiation predictions," Int. J. Fatigue, vol. 26, no. 5, pp. 463-476, 2004, doi: 10.1016/j.jffatigue.2003.10.003.

[89] S. C. Wu et al., "A physically short fatigue crack growth approach based on low cycle fatigue properties," Int. J. Fatigue, vol. 103, pp. 185-195, 2017, doi: 10.1016/j.ijfatigue.2017.05.006.

[90] S. J. Kim et al., "Cyclic Stress Response and Fracture Behaviors of Alloy 617 Base Metal and Weld Joints under LCF Loading," Adv. Mater. Sci. Eng., vol. 2015, 2015, doi: 10.1155/2015/207497.

[91] “Online Materials Information Resource - MatWeb." http://www.matweb.com/.

[92] D. R. Askeland and Pradeep P. Fulay, The science and engineering of materials, 5th ed. Thomson, 2005.

[93] R. J. Bartlett, V. F. Lotrich, and I. V. Schweigert, "Ab initio density functional theory: The best of both worlds?," J. Chem. Phys., vol. 123, no. 6, 2005, doi: 10.1063/1.1904585.

[94] L. D. Landau and E. M. Lifshitz, Theory of Elasticity Volume 7, 2nd ed. Pergamon Press.

[95] W. Callister, Fundmentals of Material Science and Engineering, 2nd ed. John Wiley \& Sons,Inc, 2005.

[96] C. Kittel, Introduction to Solid State Physics, 7th ed. John Wiley \& Sons, Inc, 1996.

[97] G. E. Totten and D. Scott MacKenzie, Handbook of Aluminum Vol.1:Physical Metallurgy and Processes, 1st ed. CRC Press, 2003. 
[98] J. J. Bikerman, "Surface Energy of Solids," basic solid state Phys., 1965, doi: https://doi.org/10.1002/pssb.19650100102.

[99] S. J. Marshall et al., "A review of adhesion science," Dent. Mater., vol. 26, no. 2, pp. 11-16, 2010, doi: 10.1016/j.dental.2009.11.157.

[100] T. Stirner, D. Scholz, and J. Sun, "Ab initio simulation of structure and surface energy of low-index surfaces of stoichiometric $\alpha$-Fe2O3," Surf. Sci., vol. 671, no. October 2017, pp. 11-16, 2018, doi: 10.1016/j.susc.2018.01.010.

[101] A. Argon, Strengthening Mechanisms in Crystal Plasticity. Oxford Scholarship, 2007.

[102] C. Li et al., "Fatigue life estimation of medium-carbon steel with different surface roughness," Appl. Sci., vol. 7, no. 4, pp. 1-12, 2017, doi: 10.3390/app7040338.

[103] R. B. Mohamed and A.K.Abdellatif, "Effect of surface finish on fatigue strength," Eng. Fract. Mech., vol. 5, no. 5, pp. 861-870, 1995.

[104] ISO 12106:2017 Metallic materials-Fatigue testing-Axial-strain-controlled method, 2nd ed. BSI, 2017.

[105] GB/T 3075-2008:Metallic Materials--Fatigue Testing--Axial-Force-Controlled Method. 2008.

[106] R. Brommesson, M. Ekh, and C. Joseph, "3D grain structure modelling of intergranular fracture in forged Haynes 282," Eng. Fract. Mech., vol. 154, pp. 57-71, 2016, doi: 10.1016/j.engfracmech.2015.12.030.

[107] J. Remacle, J. Lambrechts, and B. Seny, "Blossom-Quad: A non-uniform quadrilateral mesh generator using a minimum-cost perfect-matching algorithm," International, no. February, pp. 1102-1119, 2012, doi: 10.1002/nme.

[108] D. M. Shah and D. N. Duhl, "The Effect of Orientation, Temperature and Gamma Prime Size on the Yield Strength of a Single Crystal Nickel-Base Superalloy," pp. 105114.

[109] D. E. Pettry, N. L. Powell, and M. E. Newhouse, "Nasa Contractor Report," no. March 1984, p. 151, 1974.

[110] B. Lin, L. G. Zhao, and J. Tong, "A crystal plasticity study of cyclic constitutive behaviour, crack-tip deformation and crack-growth path for a polycrystalline nickel-based 
superalloy," Eng. Fract. Mech., vol. 78, no. 10, pp. 2174-2192, 2011, doi: 10.1016/j.engfracmech.2011.04.006.

[111] Q. Wang, G. He, and Y. Wang, "Fatigue Behavior of Aluminum Alloys under Multiaxial Loading," SAE Int. J. Mater. Manuf., vol. 7, no. 2, pp. 465-472, 2014, doi: 10.4271/2014-01-0972. 


\section{Appendix A}

The Python Scripts used for the construction of RVE are given below:

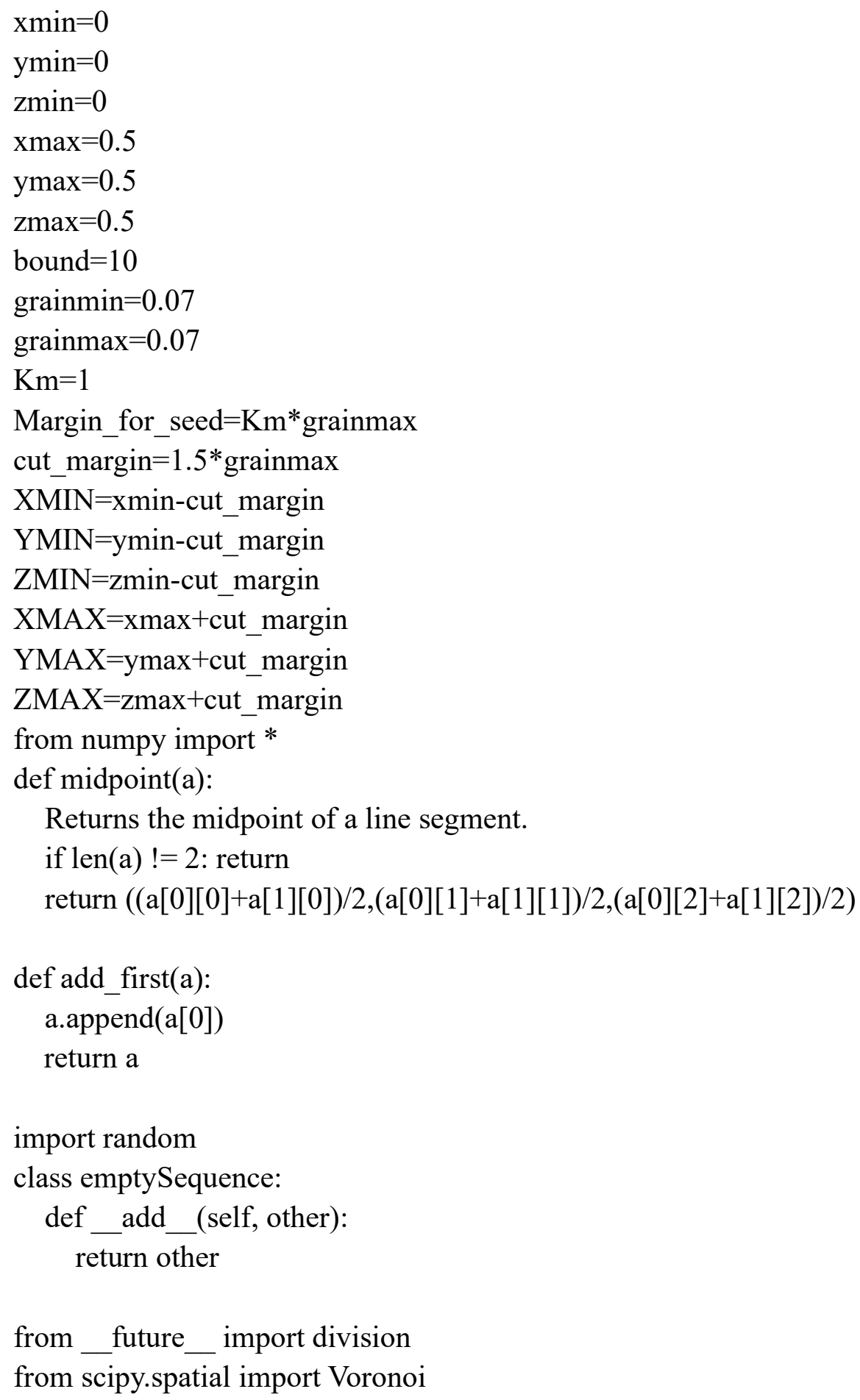




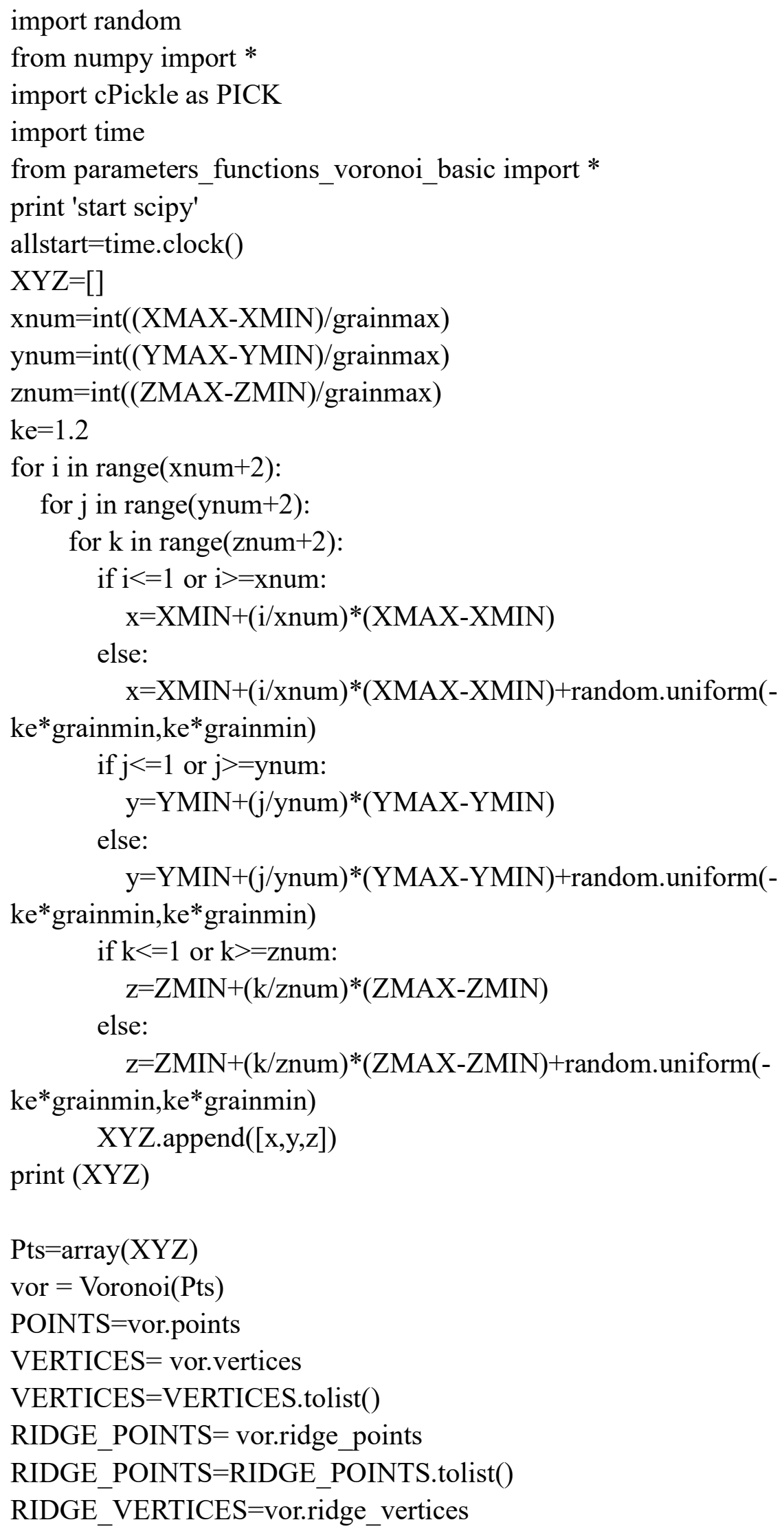


REGIONS $=$ vor.regions

POINT_REGION= vor.point_region

timer=time. $\operatorname{clock}()$

print 'voro data ok'+ "time cost: \%f s" \% (timer - allstart)

region_face=[]

dic_region_face $=\{\}$

useful_point_index $=[\mathrm{i}$ for $\mathrm{i}$ in range $(\mathrm{len}(\mathrm{XYZ}))$ if $(\mathrm{REGIONS}[\mathrm{POINT}$ REGION[i]]!=[] and (-1 not in REGIONS[POINT_REGION[i]]))]

dic_region_face $=\{$ ind:[add_first(ridge) for ridge in [[VERTICES[indexV] for indexV in RIDGE_VERTICES[ridge]] for ridge in [RIDGE_POINTS.index $(x)$ for $x$ in RIDGE_POINTS if ind in $\mathrm{x}$ ]]] for ind in useful_point_index

timer=time. $\operatorname{clock}()$

print 'voro data arrange ok '+ "time cost: \%f s" \% (timer - allstart)

PICK.dump(dic_region_face,open("voronoi-data.pkl","wb"))

print 'number of effective cells'+str(len(dic_region_face))

timer=time. $\operatorname{clock}()$

print "dump ok "+ "time cost: \%f s" \% (timer - allstart)

(The python scripts shown above are executed in the Python shell)

from __uture__import division

import traceback

import sys

from abaqus import *

from abaqusConstants import *

import mesh

import regionToolset

backwardCompatibility.setValues(reportDeprecated=False)

import cPickle as PICK

from parameters_functions_voronoi_basic import*

import time

print 'model start'

allstart=time. $\operatorname{clock}()$

dic_region_face=PICK.load(open("voronoi-data.pkl","rb"))

end $1=$ time $\operatorname{colock}()$

print "load ok" + "time cost: \%f s" \% (end1 - allstart)

mdb.Model(name='3D-Voronoi', modelType=STANDARD_EXPLICIT)

V_MODEL $=$ mdb.models['3D-Voronoi']

ROOT $=$ V_MODEL.rootAssembly 


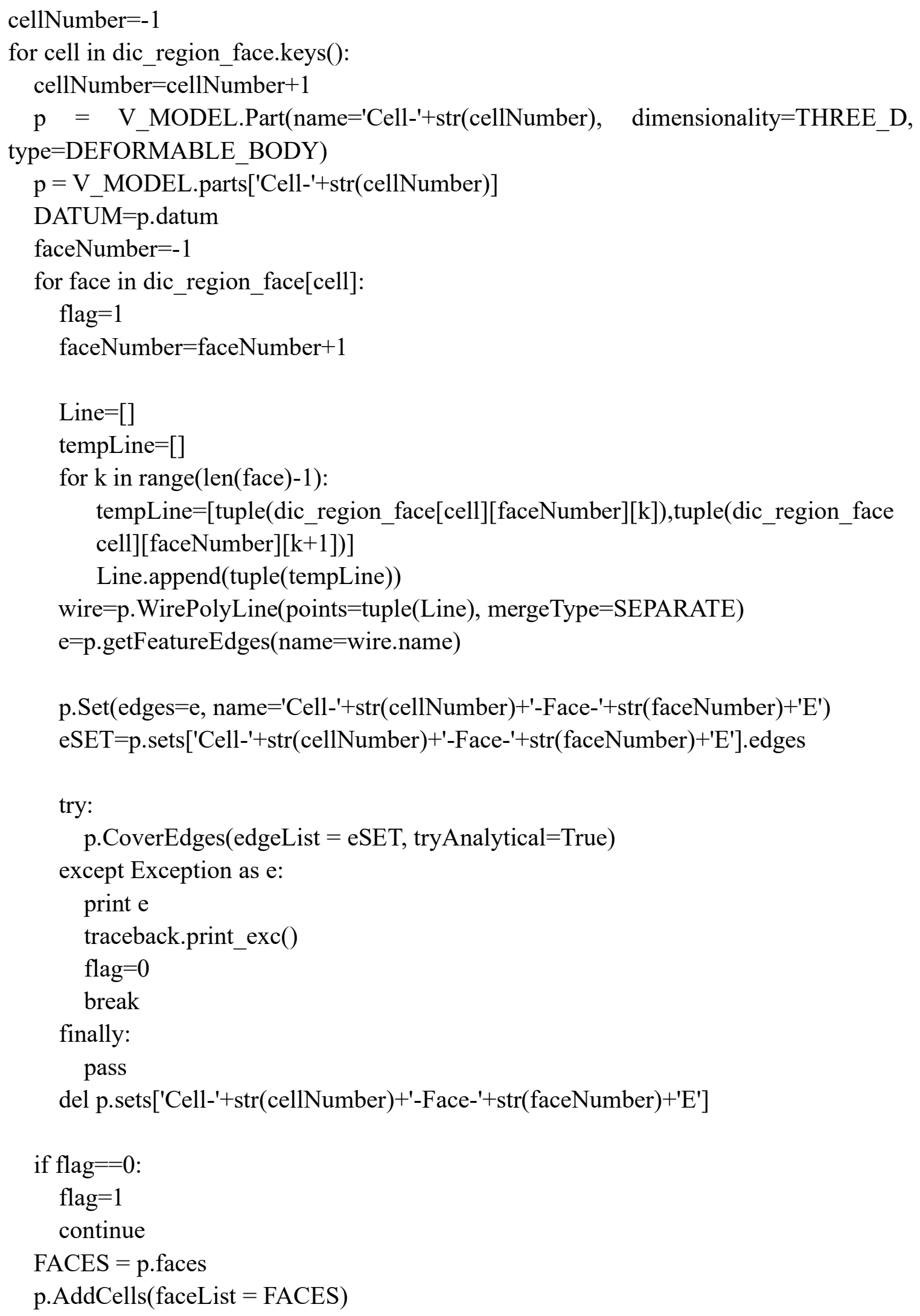


if cellNumber $\% 50==0$ :

print str(cellNumber)+'cells finished'

ROOT.DatumCsysByDefault(CARTESIAN)

for cellNumber in range(len(V_MODEL.parts)):

$\mathrm{p}=\mathrm{V} \_$MODEL.parts['Cell-'+str(cellNumber)]

ROOT.Instance $\left(\right.$ name $={ }^{\prime}$ Cell-' $+\operatorname{str}($ cellNumber $)$, art $=\mathrm{p}$, dependent $\left.=\mathrm{ON}\right)$

ROOT.InstanceFromBooleanMerge(name $=$ 'Voronoi-cells', instances $=$ tuple(ROOT.instances.values ()$)$,

keepIntersections $=\mathrm{ON}$, originalInstances $=$ DELETE, domain $=$ GEOMETRY)

ROOT.features.changeKey(

fromName='Voronoi-cells-1', toName='Voronoi-cells')

for cellNumber in range(len(V_MODEL.parts)-1):

del V_MODEL.parts['Cell-'+str(cellNumber)]

Lbox $=\mathrm{V} \_$MODEL.parts['Voronoi-cells'].cells.getBoundingBox()

LboxLow $=$ Lbox['low']

LboxHigh $=$ Lbox['high']

marginOut $=5$

$\mathrm{s}=\mathrm{V} \_$MODEL.ConstrainedSketch $($name $=$'_profile_', sheetSize $=200.0)$

$\mathrm{g}, \mathrm{v}, \mathrm{d}, \mathrm{c}=$ s.geometry, s.vertices, s.dimensions, s.constraints

s.setPrimaryObject(option $=$ STANDALONE)

s.rectangle(point1 $=($ LboxLow[0]-marginOut, LboxLow[1]-marginOut), point2 $=($ LboxHigh $[0]+$ marginOut, LboxHigh[1] + marginOut $))$

$\mathrm{p} \quad=\quad$ V_MODEL.Part(name='LBOX', dimensionality=THREE_D, type $=$ DEFORMABLE_BODY)

$\mathrm{p}=\mathrm{V} \_$MODEL.parts['LBOX']

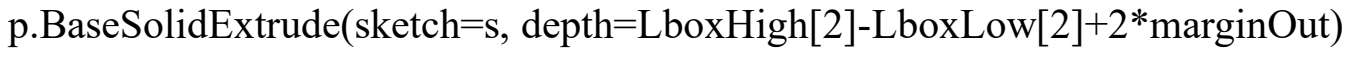

s.unsetPrimaryObject()

del V_MODEL.sketches['_profile__']

margin In $=0.1 *(\mathrm{zmax}-\mathrm{zmin})$

$\mathrm{s}=\mathrm{V} \_$MODEL.ConstrainedSketch $($name $=$'_ profile_', sheetSize=200.0)

$\mathrm{g}, \mathrm{v}, \mathrm{d}, \mathrm{c}=$ s.geometry, s.vertices, s.dimensions, s.constraints

s.setPrimaryObject(option $=$ STANDALONE)

s.rectangle $($ point $1=(x \min , y \min )$, point $2=(x \max , y \max ))$ 
$\mathrm{p}=\quad$ V_MODEL.Part(name='SBOX', dimensionality=THREE_D, type=DEFORMABLE_BODY)

$\mathrm{p}=\mathrm{V} \_$MODEL.parts['SBOX']

p.BaseSolidExtrude( sketch $=$ s, depth $=$ zmax-zmin)

s.unsetPrimaryObject()

$\mathrm{p}=\mathrm{V} \_$MODEL.parts['SBOX']

del V_MODEL.sketches['_profile_']

$\mathrm{p}=\mathrm{V} \_$MODEL.parts['SBOX']

ROOT.Instance $($ name $=$ 'SBOX', part $=\mathrm{p}$, dependent $=\mathrm{ON})$

$\mathrm{p}=\mathrm{V} \_$MODEL.parts['LBOX']

ROOT.Instance $($ name $=$ 'LBOX', part $=p$, dependent $=\mathrm{ON})$

ROOT.translate (instanceList $=($ 'SBOX', $)$, vector $=(0.0,0.0, \mathrm{zmin}))$

ROOT.translate(instanceList $=($ 'LBOX', $)$, vector $=(0.0,0.0$, LboxLow[2]-marginOut $))$

ROOT.InstanceFromBooleanCut(name='SHELL', instanceToBeCut=V_MODEL.rootAssembly.instances['LBOX'], cuttingInstances $=($ ROOT.instances['SBOX'], $)$, originalInstances $=$ SUPPRESS $)$

ROOT.features['SBOX'].resume()

ROOT.features.changeKey(fromName='SHELL-1', toName='SHELL')

ROOT.InstanceFromBooleanCut(name='marginCells', instanceToBeCut=V_MODEL.rootAssembly.instances['SBOX'], cuttingInstances $=($ ROOT.instances['Voronoi-cells'], $)$, originalInstances $=$ SUPPRESS $)$

ROOT.features.changeKey(fromName='marginCells-1', toName='marginCells')

ROOT.features['Voronoi-cells'].resume()

ROOT.InstanceFromBooleanCut(name='Voronoi-cells-trimmed', instanceToBeCut $=\mathrm{V} \_$MODEL.rootAssembly.instances['Voronoi-cells'], cuttingInstances $=($ ROOT.instances['SHELL'], $)$, originalInstances $=$ SUPPRESS $)$

ROOT.features.changeKey(fromName $=$ 'Voronoi-cells-trimmed-1', $\quad$ toName $=$ 'Voronoicells-trimmed')

ROOT.InstanceFromBooleanMerge(name='Voronoi-3D', instances $=($ ROOT.instances['Voronoi-cells-trimmed'], ROOT.instances['marginCells'],), keepIntersections $=\mathrm{ON}$, originalInstances $=$ SUPPRESS, domain $=$ GEOMETRY) ROOT.features.changeKey(fromName $=$ 'Voronoi-3D-1', toName $=$ 'Voronoi-3D')

del ROOT.features['SBOX']

del ROOT.features['LBOX']

del ROOT.features['SHELL'] 


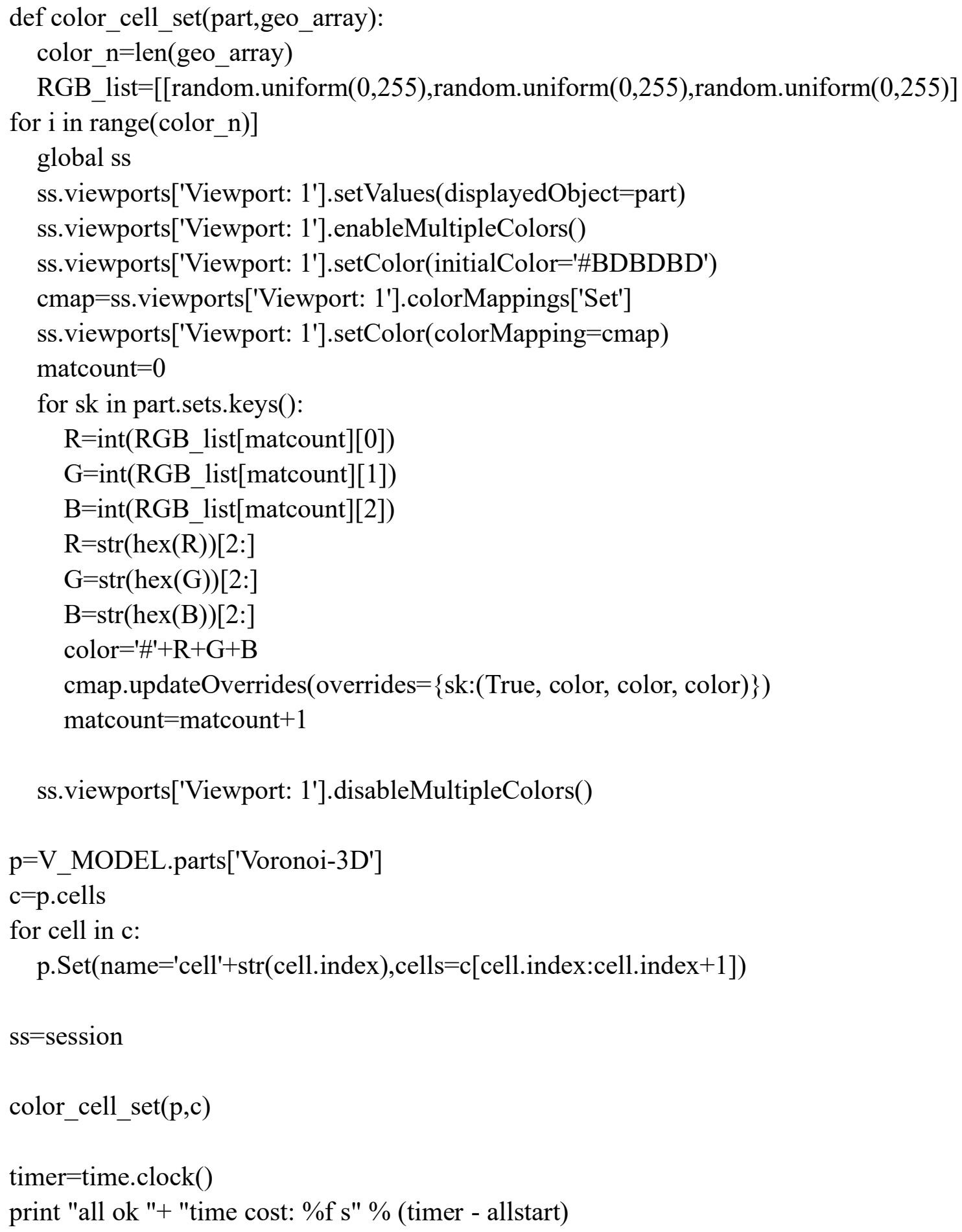




\section{Appendix B}

The Python Scripts for assigning random grain orientations to RVE are given below:

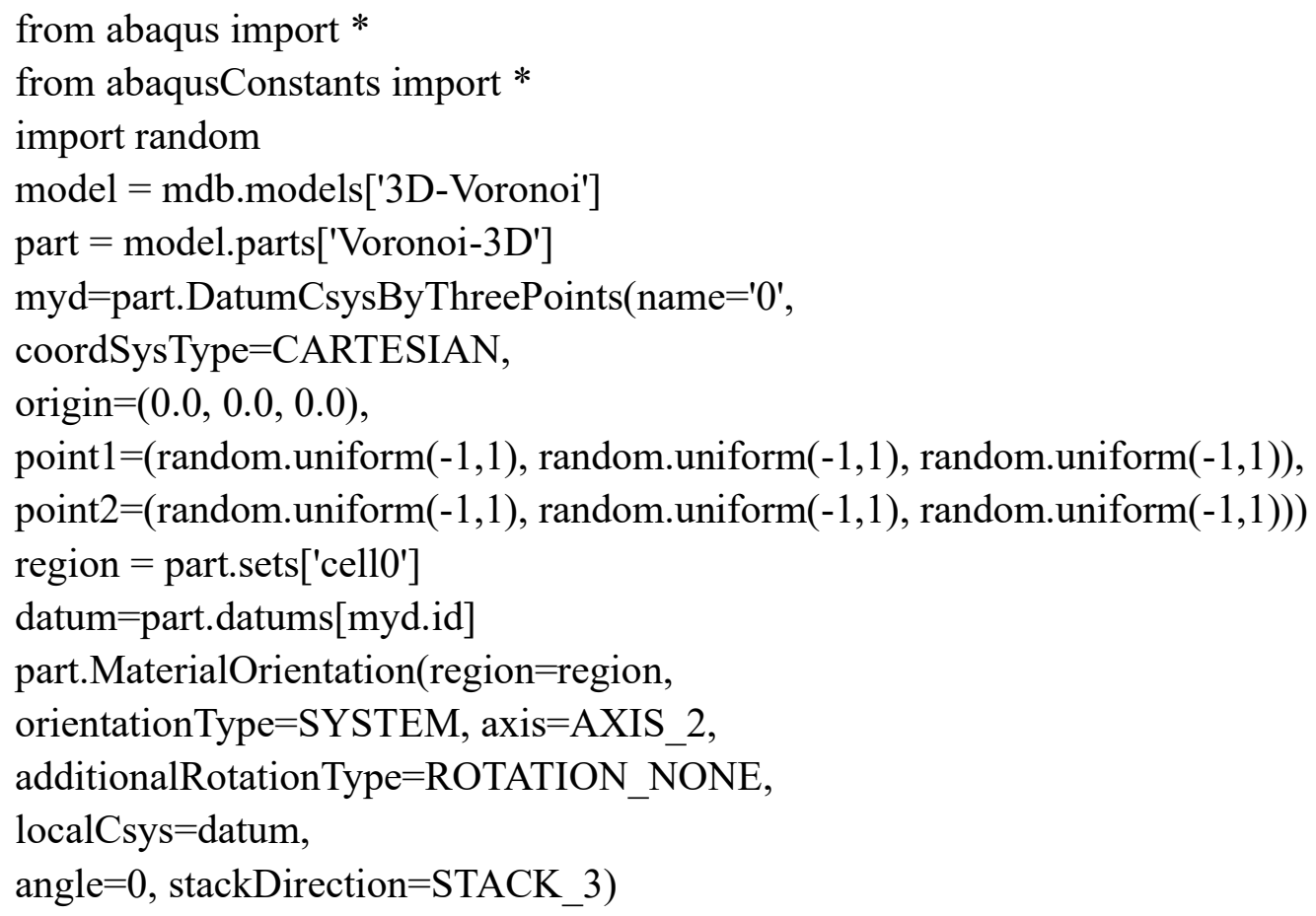

\section{Appendix C}

Matlab Codes for representation of grain orientation on virtual inverse pole figure are given below:

Startup_mtex

cs $=$ crystalSymmetry('cubic');

ss $=$ specimenSymmetry('triclinic');

fname=load('H: $\backslash$ Abaqus Data $\backslash$ HCF-415.txt');

ori $=$ orientation.byEuler(fname $(:, 1) *$ degree,fname $(:, 2) *$ degree,fname $(:, 3) *$ degree,cs,ss); plotIPDF(ori,[vector3d.X],'Markersize',8,'MarkerFacecolor','red','MarkerEdgeColor','blac $\mathrm{k}^{\prime}$ )

cs $=$ crystalSymmetry('cubic'); 
ss $=$ specimenSymmetry('triclinic');

fname=load('H:\Abaqus Data $\backslash$ HCF-415.txt');

ori $=$ orientation.byEuler(fname $(:, 1) *$ degree,fname $(:, 2) *$ degree,fname $(:, 3) *$ degree,cs,ss);

plotIPDF(ori,[vector3d.X],'all','contourf')

\section{Appendix D}

Python Scripts for extraction of PEMAG Value from Abaqus Odb are given below:

from odbAccess import*

from abaqusConstants import*

import csv

OdbFilePath='C:/temp/New Abaqus CAE/3D RVE/High Cycle Fatigue/m1/Job-5-08$\mathrm{mH1}-400-02 . \mathrm{odb}^{\prime}$

myodb $=$ openOdb(OdbFilePath)

PEMAGfield=myodb.steps['Step-Load'].frames[18].fieldOutputs['PEMAG']

vall=stressfield.values

vall nodelLabel=[]

val1PEMAGData $=[]$

for v1 in vall:

vallnodelLabel.append(v1.elementLabel)

val1PEMAGData.append(v1.data)

Z1=zip(vall nodelLabel,val1 PEMAGData)

$\mathrm{Z} 1=\operatorname{sorted}(\mathrm{Z} 1)$

sortedval1nodelLabel,sortedval1PEMAGData=zip $(* Z 1)$

myodb.close()

CsvFilePath='C:/temp/Python Script for Data Processing/Microstructure Analysis/High

Cycle Fatigue/HM1/415/F18/Job-3-08-mH1-400-02.csv'

csvFile $=$ open(CsvFilePath,'wb')

writer $=$ csv.writer(csvFile)

writer.writerow(['NodelLabel','PEMAG value'])

length $=1346296$

$\mathrm{i}=0$

while $\mathrm{i}<=$ length:

writer.writerow([sortedval1nodelLabel[i],sortedval1 PEMAGData[i]])

$\mathrm{i}=\mathrm{i}+1$

csvFile.close() 